\title{
Cleanup Verification Package for the 126-F-1, 184-F Powerhouse Ash Pit
}

\section{Prepared for the U.S. Department of Energy \\ By Washington Closure Hanford}




\section{EXECUTIVE SUMMARY}

This cleanup verification package documents completion of remedial action for the 126-F-1, 184-F Powerhouse Ash Pit. The 126-F-1 site is located within the 100-FR-2 Operable Unit in the 100-F Area of the Hanford Site in southeastern Washington State. This waste site received coal ash from the 100-F Area coal-fired steam plant that operated from 1944 to 1965. Leakage of process effluent (reactor cooling water) from the 116-F-14, 107-F Retention Basins flowed south into the ash pit, contaminating the northern portion of the ash pit. Thus, the northern and southern portions of the ash pit have been addressed separately.

Remedial action of the northern portion of the 126-F-1 site began on October 5, 2001, and was completed on June 17, 2002. Remedial action activities involved removing the contaminated coal ash from the northern portion of the ash pit and the underlying contaminated soil. All contaminated materials were disposed of at the Environmental Restoration Disposal Facility (ERDF). No remedial action was necessary in the southern portion of the 126-F-1 site but the site could not be closed out until radioactive decay occurred from the original sampling date in 2001 to 2007 to meet the radiological dose limit of $15 \mathrm{mrem} / \mathrm{yr}$ above natural background. Results of the verification sampling, laboratory analyses, and data evaluations for the 126-F-1 site indicate that all remedial action objectives and goals for direct exposure, protection of groundwater, and protection of the Columbia River have been met (see Table ES-1).

The site meets cleanup standards and has been reclassified as "interim closed out" in accordance with the Hanford Federal Facility Agreement and Consent Order (Ecology et al. 1998) and the Waste Site Reclassification Guideline TPA-MP-14, Rev. 1, (RL-TPA-90-0001) (DOE-RL 2007). A copy of the waste site reclassification form is included as Attachment ES-1. 


\section{Table ES-1. Summary of Cleanup Verification Results for the 126-F-1, 184-F Powerhouse Ash Pit.}

\begin{tabular}{|c|c|c|c|c|}
\hline $\begin{array}{l}\text { Regulatory } \\
\text { Requirement }\end{array}$ & Remedial Action Goals & Results & $\begin{array}{l}\text { Remedial } \\
\text { Action } \\
\text { Objectives } \\
\text { Attained? }\end{array}$ & Ref. \\
\hline \multirow[t]{2}{*}{$\begin{array}{l}\text { Direct Exposure - } \\
\text { Radionuclides }\end{array}$} & \multirow[t]{2}{*}{$\begin{array}{l}\text { 1. Attain } 15 \mathrm{mrem} / \mathrm{yr} \text { dose rate above } \\
\text { background for } 1,000 \text { years following } \\
\text { remediation. }\end{array}$} & $\begin{array}{l}\text { 1. Maximum dose rate calculated by } \\
\text { RESRAD for the northern portion of the } \\
\text { site is } 4.8 \mathrm{mrem} / \mathrm{yr} \text { (not accounting for } \\
\text { clean backfill). }\end{array}$ & Yes & \multirow{2}{*}{ a } \\
\hline & & $\begin{array}{l}\text { 2. Maximum dose calculated by RESRAD } \\
\text { for the southern portion of the site is } \\
\text { calculated to be } 14.3 \mathrm{mrem} / \mathrm{yr} \text { at } \\
\text { present (year 2007). }\end{array}$ & Yes & \\
\hline $\begin{array}{l}\text { Direct Exposure - } \\
\text { Nonradionuclides }\end{array}$ & 1. Attain individual COC RAGs. & $\begin{array}{l}\text { 1. There are no nonradionuclides } \\
\text { associated with this site. }\end{array}$ & N/A & N/A \\
\hline \multirow{4}{*}{$\begin{array}{l}\text { Meet } \\
\text { Nonradionuclide Risk } \\
\text { Requirements }\end{array}$} & $\begin{array}{l}\text { 1. Hazard quotient of }<1 \text { for } \\
\text { noncarcinogens. }\end{array}$ & $\begin{array}{l}\text { 1. There are no nonradionuclides } \\
\text { associated with this site. }\end{array}$ & \multirow{4}{*}{ N/A } & N/A \\
\hline & $\begin{array}{l}\text { 2. Cumulative hazard quotient of }<1 \text { for } \\
\text { noncarcinogens. }\end{array}$ & $\begin{array}{l}\text { 2. There are no nonradionuclides } \\
\text { associated with this site. }\end{array}$ & & N/A \\
\hline & $\begin{array}{l}\text { 3. Excess cancer risk of }<1 \times 10^{-6} \text { for } \\
\text { individual carcinogens. }\end{array}$ & $\begin{array}{l}\text { 3. There are no nonradionuclides } \\
\text { associated with this site. }\end{array}$ & & N/A \\
\hline & $\begin{array}{l}\text { 4. Attain a total excess cancer risk of } \\
<1 \times 10^{-5} \text { for carcinogens. }\end{array}$ & $\begin{array}{l}\text { 4. There are no nonradionuclides } \\
\text { associated with this site. }\end{array}$ & & N/A \\
\hline \multirow{4}{*}{$\begin{array}{l}\text { Groundwater/River } \\
\text { Protection - } \\
\text { Radionuclides }\end{array}$} & $\begin{array}{l}\text { 1. Attain single-COC groundwater and } \\
\text { river protection RAGs. }\end{array}$ & $\begin{array}{l}\text { 1. All single-COC groundwater and river } \\
\text { RAGs have been attained. }\end{array}$ & \multirow{4}{*}{ Yes } & $b$ \\
\hline & $\begin{array}{l}\text { 2. Attain National Primary Drinking Water } \\
\text { Standards of } 4 \mathrm{mrem} / \mathrm{yr} \text {. }\end{array}$ & $\begin{array}{l}\text { 2. All organ-specific dose rates are below } \\
\text { the } 4 \mathrm{mrem} / \mathrm{yr} \text { dose rate limit. }\end{array}$ & & $\mathrm{b}$ \\
\hline & $\begin{array}{l}\text { 3. Meet } 15 \mathrm{pCi} / \mathrm{L} \text { drinking water standard } \\
\text { for alpha emitters. }\end{array}$ & $\begin{array}{l}\text { 3. There are no alpha-emitting COCs for } \\
\text { this site }\end{array}$ & & \multirow[t]{2}{*}{$b$} \\
\hline & $\begin{array}{l}\text { 4. Meet total uranium standard of } \\
21.2 \mathrm{pCi} / \mathrm{L} .{ }^{\mathrm{c}}\end{array}$ & 4. Uranium is not a COC for this site. & & \\
\hline $\begin{array}{l}\text { Groundwater/River } \\
\text { Protection - } \\
\text { Nonradionuclides }\end{array}$ & $\begin{array}{l}\text { 1. Attain individual nonradionuclide } \\
\text { groundwater and river cleanup } \\
\text { requirements. }\end{array}$ & $\begin{array}{l}\text { 1. There are no nonradionuclides } \\
\text { associated with this site. }\end{array}$ & N/A & N/A \\
\hline \multirow{3}{*}{$\begin{array}{l}\text { Other supporting } \\
\text { Information }\end{array}$} & \multicolumn{3}{|l|}{ 1. $95 \%$ UCL calculation (Appendix C). } & $d$ \\
\hline & \multicolumn{3}{|l|}{ 2. Sample variance calculation (Appendix C). } & e \\
\hline & \multicolumn{3}{|l|}{ 3. Sample location design (Appendix C). } & f \\
\hline
\end{tabular}

a 126-F-1 RESRAD Calculation, 0100F-CA-V0146 (Northern Portion), Rev. 0, Bechtel Hanford, Inc., Richland, Washington, 0100F-CAV0102 (Southern Portion), Rev. 1, Washington Closure Hanford, Richland, Washington.

b 126-F-1 Comparison to Drinking Water Standards, 0100F-CA-V0145 (Northern Portion), Rev. 0, Bechtel Hanford, Inc., Richland, Washington, 0100F-CA-V0101 (Southern Portion), Rev. 1, Washington Closure Hanford, Richland, Washington.

' See Calculation of Total Uranium Activity Corresponding to a Maximum Contaminant Level for Total Uranium of 30 Micrograms per Liter in Groundwater, 0100X-CA-V0038 (BHI 2001)for concentration-to-activity calculation showing the $30 \mu \mathrm{g} / \mathrm{L}$ uranium MCL (40 Code of Federal Regulations 141.66) corresponds to $21.2 \mathrm{pCi} / \mathrm{L}$.

d126-F-1 Cleanup Verification 95\% UCL Calculations, 0100F-CA-V0144 (Northern Portion), Rev. 0, 0100F-CA-V0100 (Southern Portion), Rev. 0, Bechtel Hanford, Inc., Richland, Washington.

e126-F-1 Sample Variance Calculation, 0100F-CA-V0119, Rev. 0, Bechtel Hanford, Inc., Richland, Washington.

'126-F-1 Sample Design, 0100F-CA-V0061 (Overburden), Rev. 0, 0100F-CA-V0118 (Shallow), Rev. 0, Bechtel Hanford, Inc., Richland, Washington.

$\mathrm{COC}=$ contaminant of concern

$\mathrm{MCL}=$ maximum contaminant level (drinking water standard)

$\mathrm{N} / \mathrm{A}=$ not applicable
RAG = remedial action goal)

RESRAD = RESidual RADioactivity (dose model);

UCL = upper confidence limit 


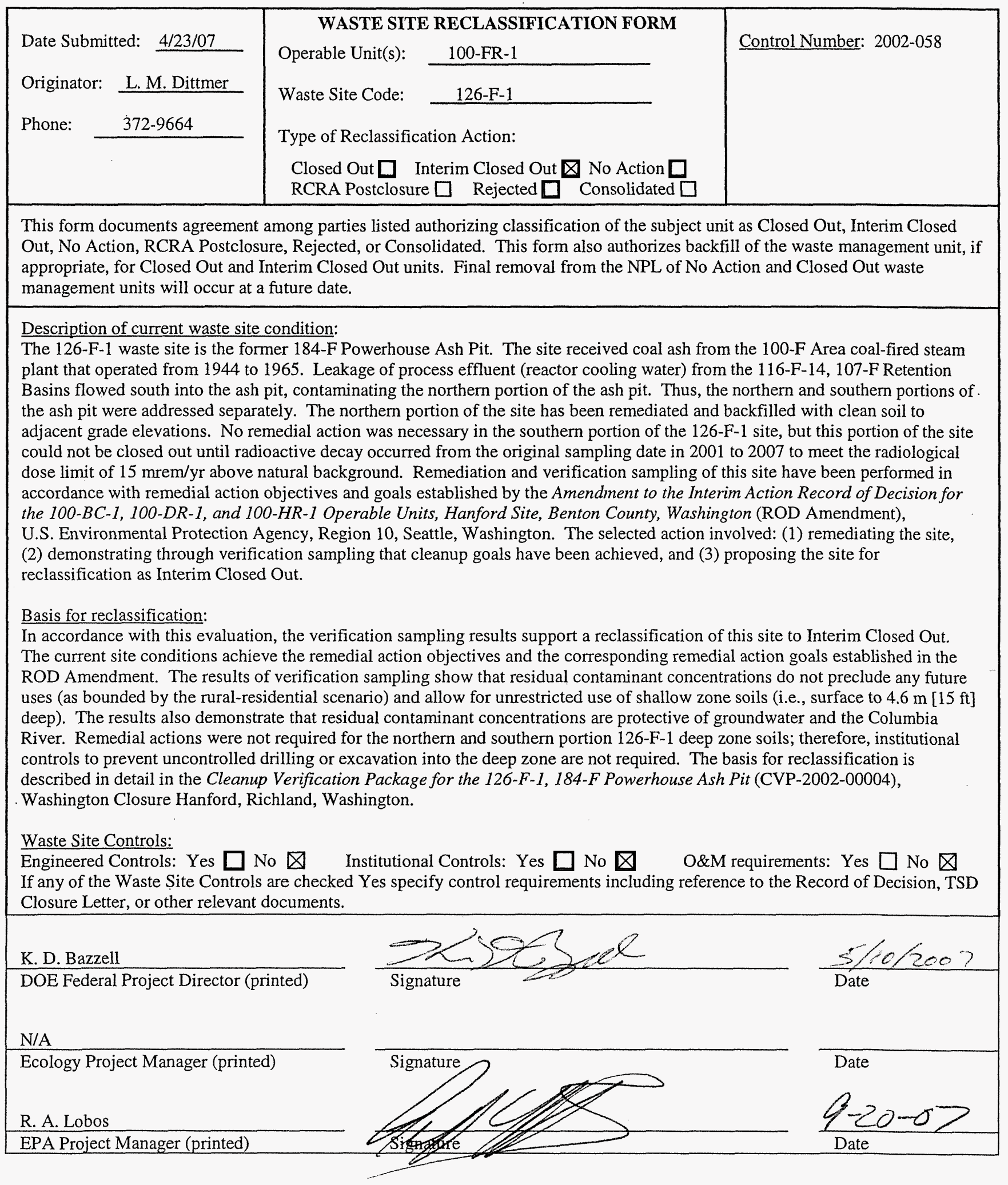


CVP-2002-00004

Rev. 1

ES-4 


\section{CONTENTS}

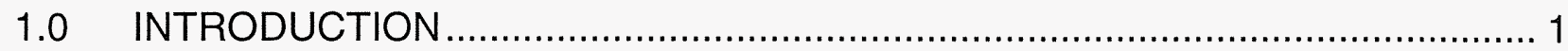

2.0 SITE DESCRIPTION AND SUPPORTING INFORMATION ............................. 4

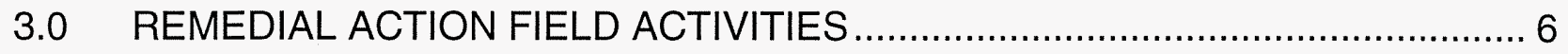

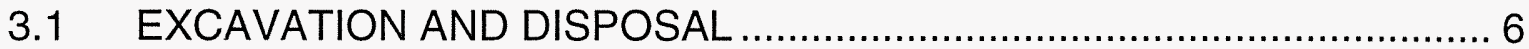

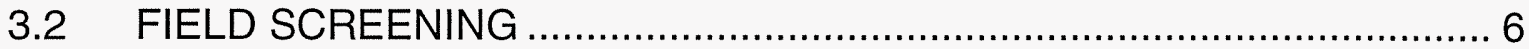

3.3 VARIANCE SAMPLING AND ANALYSIS ........................................ 10

3.4 CLEANUP VERIFICATION SAMPLING AND ANALYSIS ................... 11

4.0 CLEANUP VERIFICATION DATA EVALUATION ......................................... 12

$4.1 \quad$ DATA QUALITY ASSESSMENT PROCESS ..................................... 12

4.2 CONTAMINANTS OF CONCERN 95\% UPPER CONFIDENCE LIMIT ... 12

4.3 SITE-SPECIFIC CLEANUP VERIFICATION MODEL .......................... 14

4.4 RESRAD MODELING............................................................... 14

5.0 EVALUATION OF REMEDIAL ACTION GOAL ATTAINMENT …..................... 14

5.1 DIRECT EXPOSURE SOIL REMEDIAL ACTION GOALS ATTAINED .... 14

5.1.1 Radionuclides ...................................................................... 14

5.1.2 Nonradionuclides .................................................................. 16

5.2 GROUNDWATER REMEDIAL ACTION GOALS ATTAINED ................. 16

5.2.1 Radionuclides ................................................................... 16

5.2.2 Nonradionuclides ................................................................ 18

5.3 COLUMBIA RIVER REMEDIAL ACTION GOALS ATTAINED ............... 18

5.3.1 Radionuclides .................................................................... 18

5.3.2 Nonradionuclides ............................................................. 18

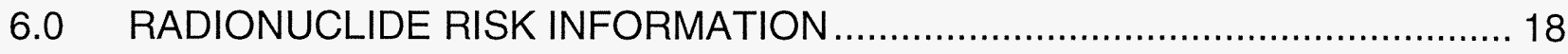

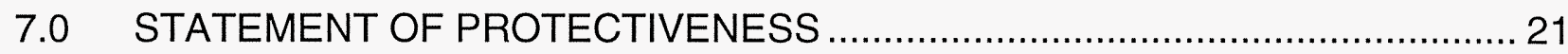

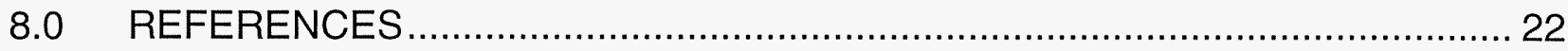




\section{APPENDICES}

A SUMMARY OF VERIFICATION SOIL SAMPLING AND ANALYTICAL

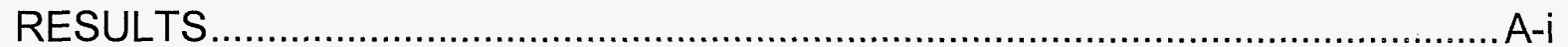

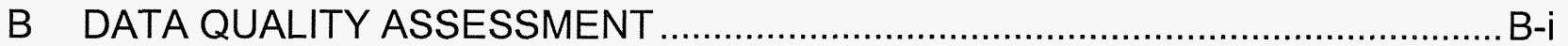

C RESRAD INPUT PARAMETERS AND CALCULATION BRIEF EXCERPTS ..........

C1 Variance Calculation, 0100F-CA-V0119 ..................................................

C2 Overburden and Shallow Zone Area Sampling Locations, 0100F-CA-V0061 (Overburden), 0100F-CA-V0118 (Shallow) …………………………..... C-16

C3 Cleanup Verification 95\% UCL Calculations, 0100F-CA-V0144 (N), 0100F-CA-V0100 (S),

C4 RESRAD Calculations, 0100F-CA-V0146 (N), 0100F-CA-V0102 (S), ........ C-51

C5 Comparison to Drinking Water Standards, 0100F-CA-V0145 (N), 0100F-CA-V0101 (S) C-82

\section{FIGURES}

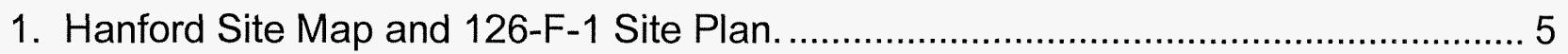

2. Pre-Remediation Topographic Plan................................................................... 8

3. Post-Remediation Topographic Plan. ................................................................ 9

4. RESRAD Analysis for the Ash Pit Northern Portion Shallow Zone - All Radionuclides, All-Pathways Dose Rate Estimate. ............................................................. 15

5. RESRAD Analysis for the Ash Pit Northern Portion Overburden - All Radionuclides,

All-Pathways Dose Rate Estimate. ............................................................... 16

6. RESRAD Analysis for the Ash Pit Southern Portion Shallow Zone All Radionuclides, All-Pathways Dose Rate Estimate........................................... 16

7. Dose Rates to Organs from Groundwater. ....................................................... 18

8. RESRAD Analysis for the Northern Portion Shallow Zone - Radionuclide Risk, All Pathways.

9. RESRAD Analysis for the Northern Portion Shallow Zone - Radionuclide Dose Rate,

All Pathways, With Corresponding Risk Values.

10. RESRAD Analysis for the Northern Portion Overburden - Radionuclide Risk, All

Pathways.

20

11. RESRAD Analysis for the Northern Portion Overburden- Radionuclide Dose Rate,

All Pathways, With Corresponding Risk Values.

12. RESRAD Analysis for the Southern Portion Shallow Zone - Radionuclide Risk, All

Pathways.

13. RESRAD Analysis for the Southern Portion Shallow Zone - Radionuclide Dose Rate,

All Pathways, With Corresponding Risk Values. 22 


\section{TABLES}

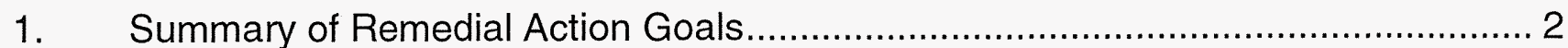

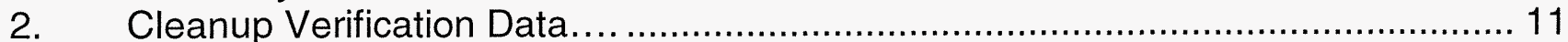

3. Estimated Peak Radionuclide Groundwater Concentrations Compared to RAGs.. 15 


\section{ACRONYMS AND ABBREVIATIONS}

$\begin{array}{ll}\text { CFR } & \text { Code of Federal Regulations } \\ \text { COC } & \text { contaminant of concern } \\ \text { COPC } & \text { contaminant of potential concern } \\ \text { CSM } & \text { conceptual site model } \\ \text { CVP } & \text { cleanup verification package } \\ \text { DCG } & \text { derived concentration guide } \\ \text { DOE-RL } & \text { U.S. Department of Energy, Richland Operations Office } \\ \text { DQA } & \text { data quality assessment } \\ \text { EPA } & \text { U.S. Environmental Protection Agency } \\ \text { ERDF } & \text { Environmental Restoration Disposal Facility } \\ \text { LCS } & \text { laboratory control spike } \\ \text { MCL } & \text { maximum contaminant level } \\ \text { MDA } & \text { minimum detectable activity } \\ \text { MS } & \text { matrix spike } \\ \text { Nal } & \text { sodium iodide } \\ \text { NCP } & \text { National Oil and Hazardous Substances Pollution Contingency Plan } \\ \text { PARCC } & \text { Precision, Accuracy, Representativeness, Comparability, } \\ & \text { and Completeness } \\ \text { PQL } & \text { practical quantitation limit } \\ \text { QA/QC } & \text { quality assurance/quality control } \\ \text { RAG } & \text { remedial action goal } \\ \text { RAO } & \text { remedial action objective } \\ \text { RDR/RAWP } & \text { Remedial Design Report/Remedial Action Work Plan } \\ \text { RESRAD } & \text { RESidual RADioactivity dose assessment model } \\ \text { ROD } & \text { Record of Decision } \\ \text { RPD } & \text { relative percent difference } \\ \text { SAP } & \text { sampling and analysis plan } \\ \text { SCA } & \text { surface contamination area } \\ \text { SDG } & \text { sample delivery group } \\ \text { TDL } & \text { target detection limit } \\ \text { UCL } & \text { upper confidence limit } \\ \text { URMA } & \text { underground radioactive material area } \\ \text { WAC } & \text { Washington Administrative Code } \\ & \end{array}$




\subsection{INTRODUCTION}

This cleanup verification package (CVP) documents that remediation goals for the 126-F-1, 184-F Powerhouse Ash Pit site have been attained in accordance with the Amendment to the Interim Action Record of Decision for the 100-BC-1, 100-DR-1, and 100-HR-1 Operable Units (ROD) (EPA 1997). Remedial action objectives and goals for the 126-F-1 site are documented in the ROD (EPA 1997) and the Remedial Design Report/Remedial Action Work Plan for the 100 Area (RDR/RAWP) (DOE-RL 2005). The ROD (EPA 1997) provides the U.S. Department of Energy, Richland Operations Office the authority, guidance, and objectives to conduct this remedial action.

Portions of the 126-F-1 Ash Pit were contaminated by unplanned releases of 105-F Reactor cooling water effluent. The northern portion of the ash pit was remediated in 2001 in accordance with the ROD (EPA 1997). The southern portion of the ash pit was sampled in 2001 and determined to meet cleanup standards through radioactive decay in 2007.

Soil cleanup levels were established in the ROD (EPA 1997) including a limited ecological risk assessment. A baseline risk assessment for the river corridor portion of the Hanford Site began in 2004, which includes a more complete quantitative ecological risk assessment. That baseline risk assessment will be used as part of a final ROD for this site.

The preferred remedy specified in the ROD (EPA 1997) and conducted for the 126-F-1 site included: (1) excavating the site to the extent required to meet specified soil cleanup levels, (2) disposing of contaminated excavation materials at the Environmental Restoration Disposal Facility (ERDF) at the 200 Area of the Hanford Site, and (3) backfilling the site with overburden and clean soil to average adjacent grade elevation. Excavation was driven by remedial action objectives for direct exposure, protection of groundwater, and protection of the Columbia River.

The contaminants of concern (COCs) for the 126-F-1 Ash Pit evolved from the time the site first appeared in the Remedial Design Report/Remedial Action Work Plan for the 100 Area (1998 RDR/RAWP) (DOE-RL 1998b) until the approval of the Sampling and Analysis Instruction for Confirmatory Sampling of the Southern Portion of the 126-F-1 Ash Pit (BHI 2001b).

The original COCs for 126-F-1 listed in the 1998 RDR include cesium-137, cobalt-60, europium-152, europium-154, europium-155, nickel-63, plutonium-239/240, strontium-90, chromium, and hexavalent chromium. The site was added to the 100 Area Remedial Action Sampling and Analysis Plan in 1998 (DOE-RL 1998a) (1998 SAP) with COCs of cesium-137 and europium-152. The list of COCs was decreased in the 1998 SAP due to findings in the Data Quality Objectives Summary Report for the 100-FR-I, 100-FR-2, 100-HR-1, 100-KR-1, and 100-KR-2 Group 4 Waste Sites (DOERL 1997) (DQO Summary) which found the other contaminants to be waste characterization concerns but not COCs. The COC list was not expanded to include nonradioactive metals because process knowledge of the site showed that 
contamination was caused by unplanned releases that were small in comparison to the total amount of 105-F Reactor cooling water effluent disposed to the 116-F-14 Retention Basins. Sampling results from related sites, as described below, also were evaluated to refine the COC list.

The 116-F-14 Retention Basins, which received the 105-F Reactor cooling water effluent, were remediated in 2001. The COCs identified through process knowledge for 116-F-14 listed in the 100 Area Remedial Action Sampling and Analysis Plan (SAP) (DOE-RL 2001a) were carbon-14, cesium-137, cobalt-60, europium-152, europium-154, europium-155, nickel-63, strontium-90, chromium (total), hexavalent chromium, and cobalt. Shallow zone cleanup verification data is presented in Table 1 showing that residual concentrations of all contaminants are below lookup values from the 100 Area RDR/RAWP (DOE-RL 2005). The lookup values provided on Table 1 represent the lowest value for each $\mathrm{COC}$ that the data is compared to (lookup values for direct exposure, groundwater protection, or protection of the Columbia River). The only exceedance is the value for total chromium, which exceeds the groundwater protection lookup value. However, fate and transport modeling results demonstrate that total chromium will not reach groundwater within 1,000 years.

These analyses demonstrate that residual concentrations of nonradionuclides in areas contaminated by reactor cooling water effluent will also be protective of human health and the environment if residual concentrations of radionuclides are below lookup values. The correlation of the levels of nonradionuclide assays to the levels of radionuclide assays at the liquid waste sites is also discussed in the SAP (DOE-RL 2001a). Based on these evaluations, the nonradionuclides (hexavalent chromium, total chromium, and cobalt) were not included as COCs. In particular, it is known that hexavalent chromium with a distribution coefficient of $0 \mathrm{~mL} / \mathrm{g}$ would have drained through the 126-F-1 coal ash and soil with the reactor cooling water while the radionuclides, which have higher distribution coefficients, would have been adsorbed by the coal ash and soil. Additionally, there is no hexavalent chromium groundwater contamination plume underlying this source unit that would indicate potential contribution from vadose zone soils. 
Table 1. 116-F-14 Cleanup Verification Data Set.

\begin{tabular}{|c|c|c|}
\hline \multirow{2}{*}{ Radionuclide COCs } & \multicolumn{2}{|c|}{ Radionuclide Activity (pCi/g) } \\
\cline { 2 - 3 } & Shallow Zone & Lookup Values $^{\text {a }}$ \\
\hline Carbon-14 & 2.0 & 5.16 \\
\hline Cesium-137 & 0.63 & 6.2 \\
\hline Cobalt-60 & 0.075 & 1.4 \\
\hline Europium-152 & 1.8 & 3.3 \\
\hline Europium-154 & 0.19 & 3.0 \\
\hline Europium-155 & 0.091 & 125 \\
\hline Strontium-90 & 0.15 & 4.5 \\
\hline Nickel-63 & 6.0 & 4,026 \\
\hline \multirow{2}{*}{ Nonradionuclide CoCs } & Nonradionuclide Concentration(mg/kg) \\
\cline { 2 - 3 } & Shallow Zone & Lookup Values $^{\text {a }}$ \\
\hline Chromium (total) & $24^{\text {b }}$ & 18.5 \\
\hline Chromium (hexavalent) & 1.2 & 2.0 \\
\hline Cobalt & 7.7 & 32 \\
\hline
\end{tabular}

\footnotetext{
a Per the Remedial Design Report/Remedial Action Work Plan for the 100 Area (DOE-RL 2005), Table 2-7.

b

RESidual RADioactivity (RESRAD) modeling using surrogate radionuclides determined that total chromium (with a distribution coefficient of $200 \mathrm{~mL} / \mathrm{g}$ ) would not reach groundwater within the period of evaluation of 1,000 years.
}

Additional information related to the 126-F-1 site is available from the Cleanup Verification Package for the 1607-F-2 Septic System (BHI 2002). A portion of 1607-F2, specifically the drain field, was located in the northern area of 126-F-1. Waste site COPCs listed in the SAP (DOE-RL 2001a) consist of cobalt-60, cesium-137, europium154, europium-155, strontium-90, uranium-234, uranium-235, uranium-238, and arsenic. Mercury was added to the COPC list based on waste characterization sampling during remediation. However, in-process sampling and analysis of the sludge in the septic tank, septic system, and tile field determined that strontium-90, the uranium isotopes, mercury, and arsenic were not COCs for the 1607-F2 site. This sampling found that residual concentrations of strontium-90, the uranium isotopes, and mercury were at levels below Hanford Site background (DOE-RL 1996). Because a large portion of the 1607-F2 drain field is within the footprint of the 126-F-1 Ash Pit, and based on agreement between the EPA and DOE, strontium-90 and nonradionuclides were not included in the final list CVP COC list for 126-F-1. Europium-152 was included in the final COC list for 1607-F2, making this list identical to the final COC list identified in the Sampling and Analysis Instruction for Confirmatory Sampling of the Southern Portion of the 126-F-1 Ash Pit (BHI 2001b)

While the SAP (DOE-RL 2001a) identified only cesium-137 and europium-152 as radionuclide COCs for $126-\mathrm{F}-1$, additional COCs were added due to process knowledge and previous data collection activities. These additional COCs were added during preparation of the Sampling and Analysis Instruction for Confirmatory Sampling of the Southern Portion of the 126-F-1 Ash Pit (BHI 2001b) which was approved by the EPA and DOE. Given the process knowledge of the minimal volume of unplanned reactor 
effluent leaks into the site, combined with the information from concurrent sampling of the 1607-F2 and 116-F-14 sites, the EPA and DOE determined that there was not a need to add additional, nonradioactive COCs for the 126-F-1 site.

The COCs for the 126-F-1 Ash Pit waste site consist of the following:

- Cesium-137

- Cobalt-137

- Europium-152

- Europium-154

- Europium-155.

\subsection{SITE DESCRIPTION AND SUPPORTING INFORMATION}

The 126-F-1 site is part of the 100-FR-2 Operable Unit in the 100-F Area. The 126-F-1 site is approximately $220 \mathrm{~m}$ (720 ft) east of the 105-F Reactor Building and $315 \mathrm{~m}$ $(1,033 \mathrm{ft}$ ) from the Columbia River (Figure 1). The 126-F-1 site was created as a result of disposal of ash from the 184-F coal-fired steam plant that operated between 1944 and 1965. Large but unquantified amounts of coal ash were sluiced to the ash pit with raw river water through a $20 \mathrm{~cm}$ (8 in.) pipe. Leakage of reactor cooling water from the 116-F-14, 107-F Retention Basins flowed into the northern portion of the ash pit, and was contained with an earthen dike. The coal ash sluice pipe was extended through the dike in the late 1940s to a point farther south, establishing coal ash discharge to the southern portion of the coal ash pit area.

After the 105-F Reactor was shut down in 1965, the entire ash pit was posted as a "surface contamination area" and then, later, as a "contamination area." As noted in the 126-F-1 Radiological Investigation and Downposting Final Report (BHI 1996), routine surveillance conducted at the site as part of the Radiation Area Action project, revealed that the southern portion was improperly posted as a "contamination area." Posting of the entire ash pit as a "contamination area" had occurred out of concern that heavy equipment used to level the ash may have spread contamination throughout the entire site. In 1996, test pits were dug (BHI 1996) and radiological surveys of the entire ash pit were performed, which resulted in downposting the northern section of the pit from a "surface contamination area" to an "underground radioactive material area" and the southern section from a "surface contamination area" to no posting at all (BHI 2001b).

The area of the 1607-F2 Septic System site was contained within the boundary of the 126-F-1 Ash Pit and was excavated with the northern portion, but sampled separately. Cleanup verification of the 1607-F2 waste site is addressed in a separate CVP document (BHI 2002). 
Figure 1. Hanford Site Map and 126-F-1 Site Plan.

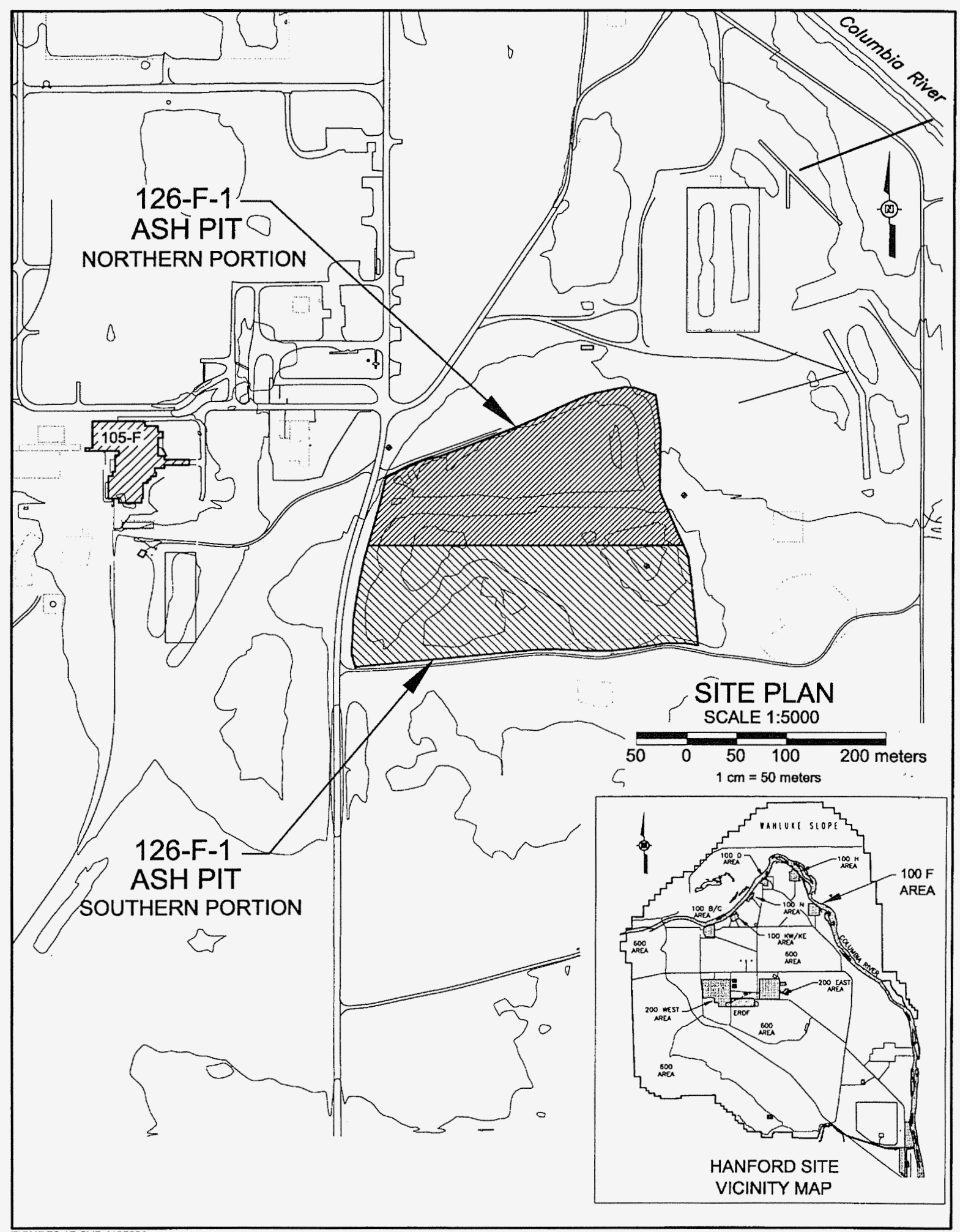




\subsection{REMEDIAL ACTION FIELD ACTIVITIES}

\subsection{EXCAVATION AND DISPOSAL}

Remedial action at the 126-F-1 site began on October 5, 2001. Excavation of the site involved removing the overburden materials and underlying contaminated soil in the northern portion of the site only. Based on field screening (discussed in Section 3.2), overburden materials identified as potentially clean were placed in a stockpile for potential use as backfill. Contaminated materials were disposed of at ERDF.

On June 17, 2002, the excavation of the northern portion of the site was completed. Pre- and post-remediation topographic maps are shown in Figures 2 and 3 , respectively. The average elevation of the bottom of the excavation was at $119.8 \mathrm{~m}$ (393.0 ft) upon completion. The excavation was approximately $10,689 \mathrm{~m}^{2}\left(115,052 \mathrm{ft}^{2}\right)$ in area with a maximum depth of approximately $4 \mathrm{~m}$ (13 ft). Approximately 100,964 metric tons $(111,313$ tons) of material from the site were disposed of at ERDF.

The Data Quality Objectives Summary Report for the 100-FR-I, 100-FR-2, 100-HR-1, 100-KR-1, and 100-KR-2 Group 4 Waste Sites (DOE-RL 1997) identified the 126-F-1 southern portion of the site as a "Type III" site for which sampling is required to identify the nature/extent of contamination or to verify the need for remediation. Discussion with the regulators supported the use of the Sampling and Analysis Plan for the 100 Area Remaining Sites (Remaining Sites SAP) (DOE-RL 2000) confirmatory sampling designs to verify that the southern portion of the ash pit met the cleanup standards and that no further remediation was necessary.

\subsection{FIELD SCREENING}

In the northern portion of the 126-F-1 site, field screening was conducted during the site remedial action as specified in the SAP (DOE-RL 2001). Field screening was used to guide the excavation to quickly assess for the presence and level of contamination. Field screening for the 126-F-1 site included using a radiological data mapping system survey, hand-held sodium iodide ( $\mathrm{Nal}$ ) detectors, and gamma energy analyses. The radiological mapping survey is performed over more than $50 \%$ of the site excavation surface area. The hand-held Nal detector is used to screen excavated waste material and to screen for potential excavation wall and floor hot spots. Gamma energy analyses were used to support waste characterization and to corroborate the radiological mapping survey and hand-held $\mathrm{Nal}$ detector data.

The Sampling and Analysis Instruction for Confirmatory Sampling of the Southern Portion of the 126-F-1 Ash Pit (BHI 2001b) summarizes field screening activities and data for the southern portion of the site. The document describes the following activities for the southern portion: 
Radiological surveys of the southern portion were conducted in 1995 to support selection of remedial action alternatives for the site (BHI 1995), and identified mostly low-level contamination throughout the site. Radiological surveys, using sodium iodide (Nal) detectors and alpha energy and gamma energy analyses, were performed in March 1996 to support downposting of the southern portion of the site. Six test pits were excavated, and samples of ash were analyzed onsite using high-purity germanium and gamma spectroscopy during the March 1996 data collection activities. Results of this effort indicated that slightly elevated levels of cobalt-60, cesium-137, europium-152,

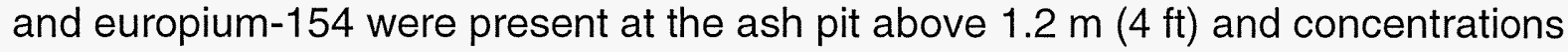
decreased with depth (BHI 2001b).

In 1997, Group 4 Remedial Action performed a field investigation to evaluate if sufficient information existed to support remedial design and to fill in data gaps for some of the 100 Area waste sites (Blumenkranz 1997). As part of this activity, a test pit was excavated on the south side of the ash pit to evaluate the density of the ash for development of remedial action contract specifications. This investigation identified a coal ash layer of varying depth with a density of $999 \mathrm{~kg} / \mathrm{m}^{3}$ and the underlying soil (i.e., sand and gravel) with a density of $2,093 \mathrm{~kg} / \mathrm{m}^{3}$ (BHI 2001b).

In 1999, spectral-gamma logging using a small-diameter geophysical logging system was performed to collect data on the distribution of subsurface gamma-emitting radionuclides in the vadose zone. The gross gamma-logging data indicated elevated gamma activity in the upper few feet of each hole, generally with a peak in activity at approximately $0.5 \mathrm{~m}(1.5 \mathrm{ft})$. It was initially thought that the elevated total gamma readings (although low) represented widespread low-level contamination from the reactor effluent leakage that had mixed with powerhouse coal ash. However, after spectral gamma analysis of the data, it was determined that the increase was primarily from the naturally occurring radionuclides of potassium, uranium, and thorium. Minor concentrations of cesium-137 and cobalt- 60 were determined from the spectral gamma logs for a few of the probe holes. The concentrations above minimal detectable activity levels were located within the top $1.5 \mathrm{~m}(5 \mathrm{ft})$ of the surface material. The majority of analyses from the southern portion of the site were below detection limits (BHI 2001b).

A review of the combined historical radiological field survey data, GeoProbe gamma survey data, and high-purity germanium data for test pit samples indicated that confirmatory sampling should be performed to provide evidence that the southern portion of the ash pit could be closed without remediation (BHI 2001b). 


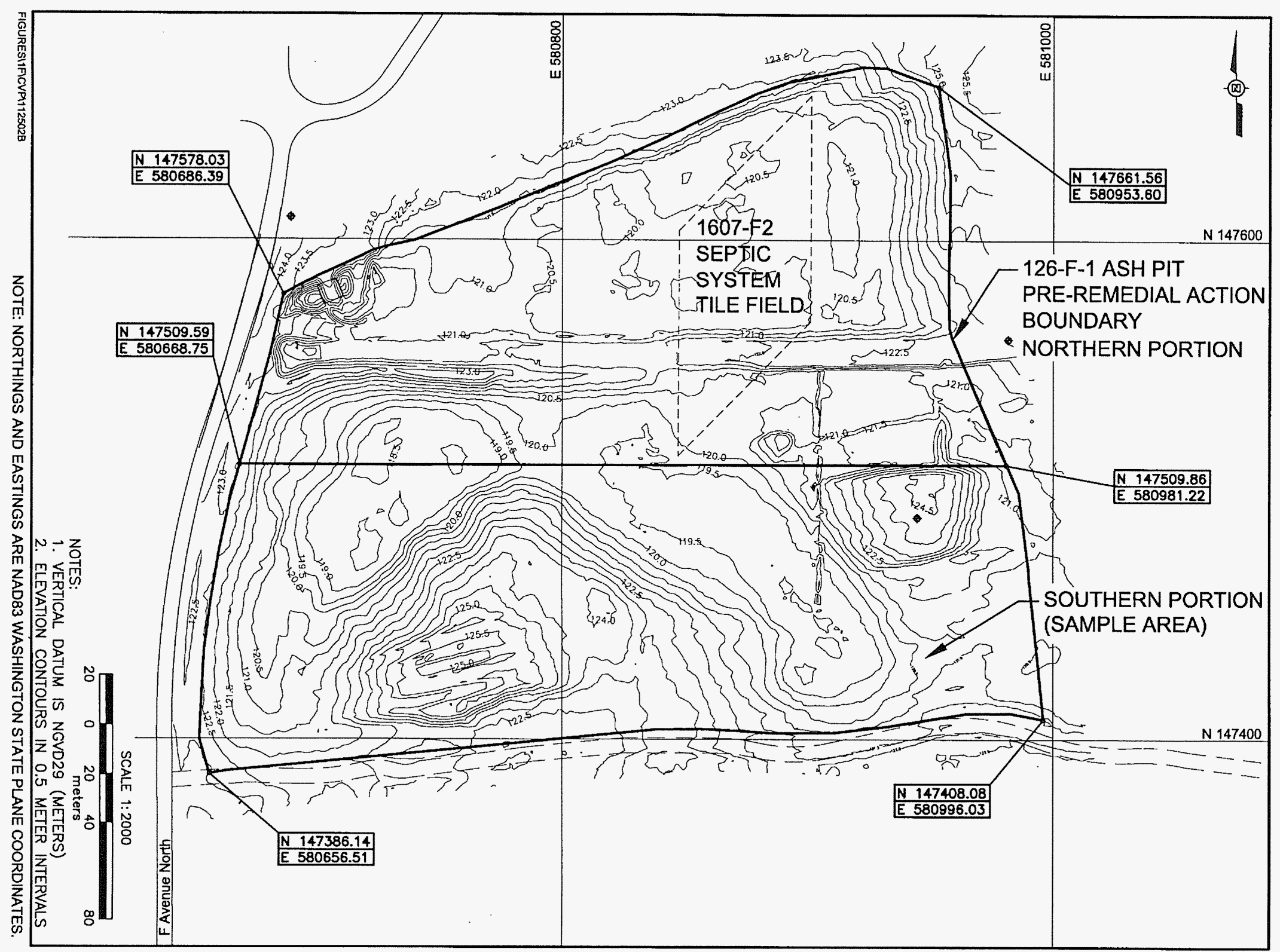

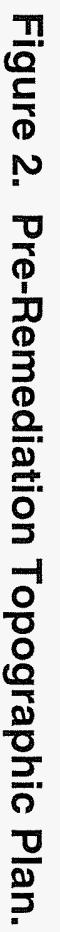




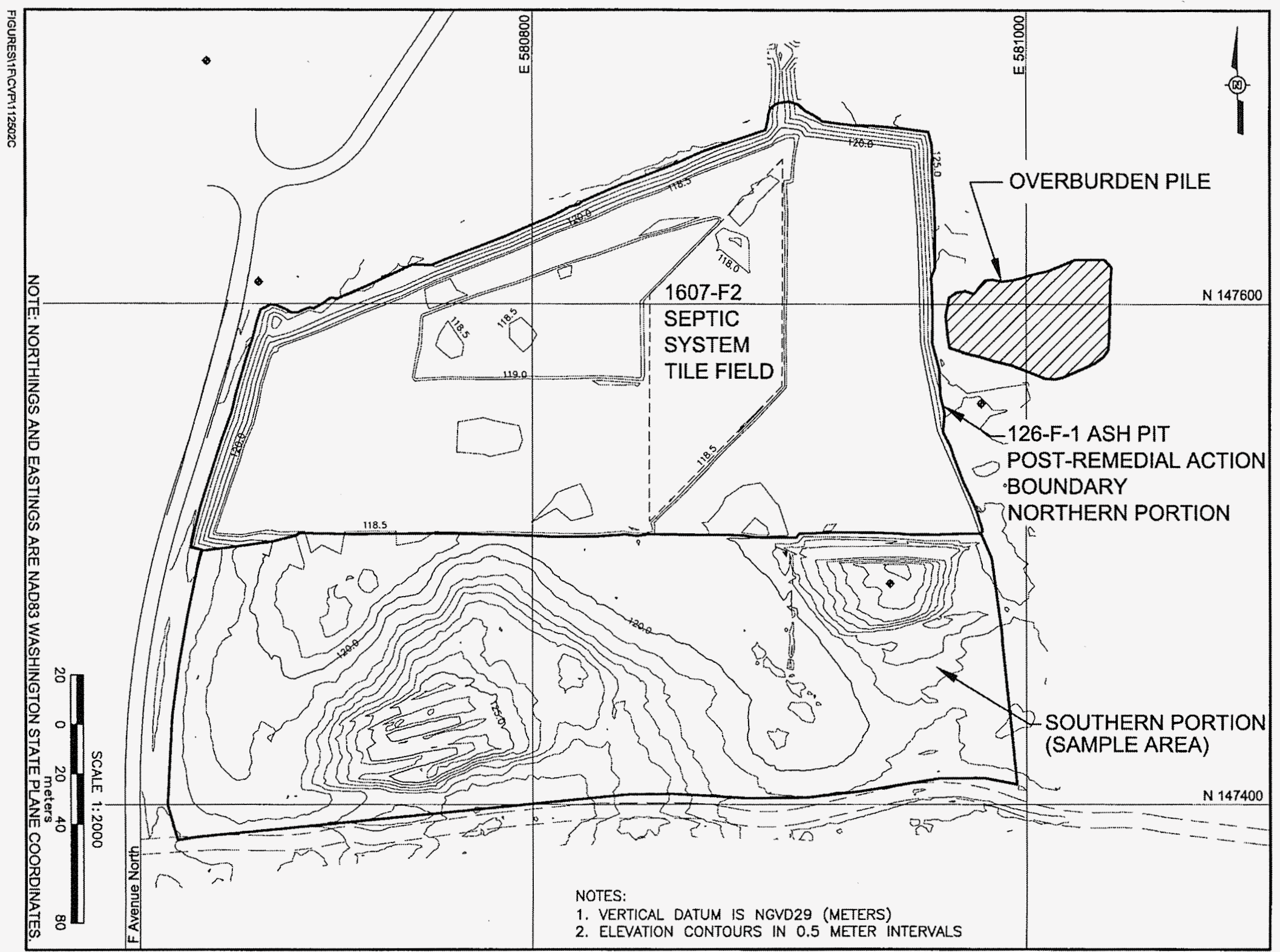

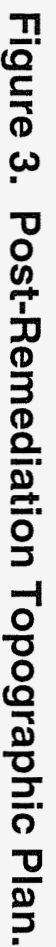




\subsection{VARIANCE SAMPLING AND ANALYSIS}

Variance analysis was performed in the northern portion of the site following field screening. The variance analysis quantifies the variability of residual contamination. This information was used to determine the site-specific number of final cleanup verification samples to be collected in the northern portion of the site. Variance sampling and testing was performed on March 6, 2002. As specified in the SAP and the instruction guide (DOE-RL 2001, BHI 2001a), variance analysis was performed for the shallow zone and overburden decision units. A total of 216 variance samples were collected from the northern portion of the 126-F-1 site. Variance samples were collected in the locations shown on the 126-F-1 Ash Pit Shallow Zone Sampling Design and 126-F-1 Overburden Sampling Design calculation briefs (Appendix C).

The results of the variance analysis indicated that the number of verification samples to be taken from each decision subunit in the northern half of the site was less than the default number of four; therefore, four final verification samples were collected from each northern shallow zone decision subunit.

Using the field investigation data and the history of the site, the surface spill conceptual site model (CSM) was selected to most likely approximate the distribution of contamination in the southern portion of the 126-F-1 site. Therefore, the confirmatory sampling design, and number of confirmatory samples required, was based on the requirements specified in the Remaining Sites SAP, Appendix B, "Sampling Design for the Surface Spills Conceptual Site Model" (DOE-RL 2000). In addition, historical data were available to evaluate site and sampling variability in the southern portion of the 126-F-1 site. Specifically, test pit data obtained during sampling activities in March of 1996 for cobalt-60 and cesium-137 were used (BHI 2001b).

The first step of the surface spill CSM sample design (DOE-RL 2000) establishes the number of lateral sampling locations in the $X-Y$ plane. Because mechanical mixing of the spill-contaminated ash occurred, a systematic grid sampling design was recommended (rather than a random sample design) to ensure that there were no significant areas of elevated concentrations above cleanup levels existing within the site. Therefore, the sample design selected was a random-start, systematic grid sample design that ensured a $95 \%$ probability of detecting a hot spot and would also support statistical treatment of the sample results. The size of a significant hot spot was set at a circle with a $22 \mathrm{~m}(72.2 \mathrm{ft})$ radius, which is smaller than the 0.2 hectare $(0.5 \mathrm{acre})$ area considered by the EPA to be of a sufficient size to pose a potential human health risk in a residential setting (EPA 1996). Based upon these considerations, Visual Sample Plan ${ }^{1}$ was used to develop the sampling design. A total of 24 samples were systematically collected on a $42 \mathrm{~m}$ (137.8 ft) triangular grid, having a 95\% probability that a hot spot with a $22 \mathrm{~m}$ ( $72.2 \mathrm{ft})$ or greater radius would be detected.

\footnotetext{
${ }^{1}$ Visual Sampling Plan is a site map-based user-interface program that may be downloaded from the Internet at http://dqo.pnl.gov.
} 


\subsection{CLEANUP VERIFICATION SAMPLING AND ANALYSIS}

Final cleanup verification samples were collected from the northern portion of the site following variance sampling, analysis, and data evaluation. Cleanup verification sampling in the northern portion of the site began on July 16, 2002, and concluded on July 17,2002 . The samples were submitted to offsite laboratories for analysis using approved EPA analytical methods, as required per the SAP (DOE-RL 2001). Each verification sample was a composite formed by combining samples collected at four randomly selected nodes within each sampling area (excluding the quality assurance/quality control samples). The sample design methodology and sample location figures are presented in the calculation briefs for variance analysis and sample design in Appendix C.

The division of the site excavation into decision units, as shown on the sample design figures in Appendix $\mathrm{C}$, is a function of the applicable RAGs. The direct exposure, groundwater protection, and river protection RAGs are applicable to soils within $4.6 \mathrm{~m}$ $(15 \mathrm{ft}$ ) of the ground surface (shallow zone and overburden soil). The northern portion of the 126-F-1 site consisted of shallow zone and overburden decision units. The site

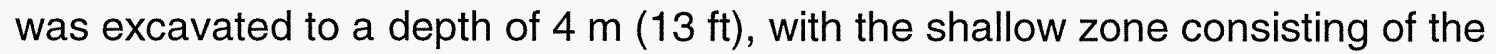
excavation sidewalls together with the floor of the excavation.

As described in Section 3.3, the required number of composite samples for each decision subunit in the shallow zone was less than the default number of four composite samples specified in the SAP (DOE-RL 2001). Therefore, the default number of four samples were collected from each shallow zone decision subunit (excluding the quality assurance/quality control samples). The shallow zone decision unit for the northern portion of the site contained nine decision subunits, which were each divided into four sampling areas, giving a total of 36 sampling areas. Each sampling area was further divided into 16 sampling nodes. Four of the 16 nodes were randomly selected and sampled to form a composite sample for each sampling area.

An overburden decision unit was included with this site. The overburden decision unit for the northern portion of the site contained three decision subunits, which were each divided into four sampling areas, giving a total of 12 sampling areas. One composite cleanup verification sample from each sampling area was obtained by compositing the samples collected from four of the 16 sampling nodes for each sample area. The sample design for this site is documented in the sample area and location calculation brief included in Appendix C.

Confirmatory samples were collected from the southern portion of the site after a review of the combined historical radiological field survey data, GeoProbe gamma survey data, and high-purity germanium data for test pit ash samples indicated that confirmatory sampling should be performed to provide evidence that the southern portion of the ash pit can be closed without remediation. The site conditions, site COCs, and the CSM are used to determine the number of confirmatory samples required (as described in Section 3.3), the sampling depths, sample type, and sample locations. A complete description of the confirmatory sampling design basis for the southern half of the 
126-F-1 is contained in Appendix A of the Sampling and Analysis Instruction for Confirmatory Sampling of the Southern Portion of 126-F-1 Ash Pit (BHI 2001b).

Confirmatory sampling in the southern portion of the site began May 21, 2001, and concluded on June 28,2001 . Samples of the ash were collected as composite samples from 0 to $0.6 \mathrm{~m}(2 \mathrm{ft})$ below ground surface at each of 24 grid sample locations, as described in Section 3.3. These samples were analyzed at an offsite laboratory.

\subsection{CLEANUP VERIFICATION DATA EVALUATION}

This section presents the evaluation and modeling of the 126-F-1 cleanup verification data for comparison with the data quality criteria and RAGs.

\subsection{DATA QUALITY ASSESSMENT PROCESS}

A data quality assessment is performed to compare the verification and confirmatory sampling approach and resulting analytical data with the sampling and data quality requirements specified by the project objectives and performance specifications.

The data quality assessment for the 126-F-1 site determined that the data are of the right type, quality, and quantity to support site verification decisions within specified error tolerances. All verification and confirmatory data were found to be acceptable for decision-making purposes. The evaluation verified that the sample design was sufficient for the purpose of clean site verification. The cleanup verification sample analytical data are stored in the Hanford Environmental Information System and are summarized in Appendix A. The detailed data quality assessment is presented in Appendix B.

\subsection{CONTAMINANTS OF CONCERN 95\% UPPER CONFIDENCE LIMIT}

The primary statistical calculation to support cleanup verification is the $95 \%$ UCL on the arithmetic mean of the data. The $95 \%$ UCL values for each COC are computed for each decision unit (for the shallow zone and overburden). Prior to calculating the $95 \%$ UCL, the individual sample results are reviewed and, as appropriate, adjusted per the SAP (DOE-RL 2001). This process is summarized below.

Verification sampling summary statistics (95\% UCL values) are listed in Table 2. Individual sample cleanup verification results are presented in Appendix A.

- Radionuclides: The laboratory reported value is used in the calculation of the $95 \%$ UCL. In cases where the laboratory does not report a value for data qualified with a "U" (i.e., less than the detection limit), half of the minimum detectable activity is used in the calculation of the $95 \% \mathrm{UCL}$. 
- Nonradionuclides:

There are no nonradionuclide COCs associated with this site.

Table 2. Cleanup Verification Data.

\begin{tabular}{|c|c|c|c|c|c|}
\hline \multirow[b]{2}{*}{ cocs } & \multicolumn{2}{|c|}{ 95\% UCL Statistical Values } & \multirow{2}{*}{$\begin{array}{l}\text { Hanford Site } \\
\text { Background }^{\text {a }}\end{array}$} & \multicolumn{2}{|c|}{ Cleanup Verification Data Set } \\
\hline & $\begin{array}{c}\text { Shallow } \\
\text { Zone }\end{array}$ & Overburden & & $\begin{array}{c}\text { Shallow } \\
\text { Zone }\end{array}$ & Overburden \\
\hline \multicolumn{6}{|c|}{ Northern Portion Radionuclides $(\mathrm{pCi} / \mathrm{g})^{\mathrm{c}}$} \\
\hline Cesium-137 & 0.39 & 0.097 & 1.1 & 0.39 & $0(<B G)^{b}$ \\
\hline Cobalt-60 & 0.041 & 0.035 & 0.008 & 0.041 & $0.027^{\mathrm{b}}$ \\
\hline Europium-152 & 0.66 & 0.29 & $N A^{d}$ & 0.66 & $0.29^{\mathrm{b}}$ \\
\hline Europium-154 & 0.091 & 0.077 & 0.033 & 0.091 & $0.044^{\mathrm{b}}$ \\
\hline Europium-155 & 0.12 & 0.054 & 0.054 & 0.12 & $0.00028^{b}$ \\
\hline \multicolumn{6}{|c|}{ Southern Portion Radionuclides (pCi/g) ${ }^{c}$} \\
\hline Cesium-137 & 0.39 & \multirow{5}{*}{$\mathrm{N} / \mathrm{A}$} & 1.1 & $O(<B G)^{b}$ & \multirow{5}{*}{ N/A } \\
\hline Cobalt-60 & 0.27 & & 0.008 & $0.26^{\mathrm{b}}$ & \\
\hline Europium-152 & 3.5 & & $N A^{d}$ & $3.5^{\mathrm{b}}$ & \\
\hline Europium-154 & 0.45 & & 0.033 & $0.42^{b}$ & \\
\hline Europium-155 & 0.11 & & 0.054 & $0.061^{b}$ & \\
\hline
\end{tabular}

${ }^{a}$ Represents the $90^{\text {th }}$ percentile of the lognormal distribution (DOE-RL 1996).

${ }^{b}$ For overburden and unexcavated soil in the southern portion of the waste site, anthropogenic background (global fallout from nuclear weapons testing) and naturally occurring background is subtracted from all radionuclides. Refer to Appendix $\mathrm{C}$ for additional details on determination of statistical values.

${ }^{\mathrm{C}}$ Laboratory data including the minimum detectable activity or practical quantitation limit for the individual cleanup verification samples are included in Appendix A and the 95\% UCL calculation brief.

${ }^{\mathrm{d}} \mathrm{A}$ background value for this analyte has not been established.

$B G=$ background

$\mathrm{COC}=$ contaminant of concern

$\mathrm{N} / \mathrm{A}=$ not applicable. ${ }^{*} T$ The southern portion of the site had no associated overburden decision unit.

$\mathrm{UCL}=$ upper confidence limit

Statistical calculations are presented in the $126-\mathrm{F}-1$ cleanup verification $95 \% \mathrm{UCL}$ calculation brief (Appendix C). The columns on the left side of Table 2 are the $95 \%$ statistical values before subtraction of background. The columns on the right side of the table present statistical values adjusted for background. For overburden soil, anthropogenic background (global fallout from nuclear weapons testing, DOE-RL 1996) and naturally occurring background is normally subtracted from all radionuclides, as was done for the northern portion of the site. In the southern portion, Hanford Site background values were also subtracted, since the soil was not excavated and would be impacted by anthropogenic and naturally occurring background concentrations as well. Refer to Appendix $\mathrm{C}$ for additional details on determination of statistical values. It is these values that constitute the cleanup verification data set and are used for RESidual RADioactivity (RESRAD) modeling. 


\subsection{SITE-SPECIFIC CLEANUP VERIFICATION MODEL}

The site-specific cleanup verification models used for both the northern and southern portions of the site were developed using the statistical values summarized above (Table 2). The models used assume the worst case described in the RDR/RAWP (DOE-RL 2005), where the excavation statistical concentrations for the shallow zone decision units continue at the same concentrations to groundwater. Schematic cross sections of the site-specific cleanup verification models are included in the RESRAD calculations in Appendix C.

\subsection{RESRAD MODELING}

The individual radionuclide cleanup verification statistical values for each portion of the site (Table 2) were entered into the RESRAD computer code, Version 6.2 (ANL 2002) for the northern portion or Version 6.3 (ANL 2005) for the southern portion, to predict the dose rate and to predict the impact on groundwater and the river from residual $\mathrm{COC}$ concentrations. Soil concentration values for the southern portion from the 126-F-1 cleanup verification 95\% UCL calculation brief (Appendix C) are reduced by radioactive decay calculations in the RESRAD calculation brief for the southern portion (Appendix C) from original sample values on 5/21/2001 to present decayed values (2/8/2007).

The RESRAD modeling methodologies, results, input values, and the site-specific cleanup verification models for the northern and southern portions of the site are included in the RESRAD calculation briefs (Appendix C). The drinking water dose rate calculations based on the RESRAD-predicted groundwater radionuclide concentrations are shown in the comparison to drinking water standards calculation briefs for each portion of the site as well (Appendix $C$ ). Specific results from the calculations are discussed in the RAG evaluation section (Section 6.0).

\subsection{EVALUATION OF REMEDIAL ACTION GOAL ATTAINMENT}

This section demonstrates that remedial actions at the 126-F-1 site have achieved the applicable RAGs. Sections 5.1, 5.2, and 5.3 address attainment of direct exposure, groundwater protection, and Columbia River protection RAGs, respectively.

\subsection{DIRECT EXPOSURE SOIL REMEDIAL ACTION GOALS ATTAINED}

\subsubsection{Radionuclides}

The results of the RESRAD all-pathways scenario dose rate estimate for the northern and southern portion of the site are presented in Figures 4,5 , and 6 . This dose rate represents the dose contributions from soils at relevant time periods. 
The dose rate was largest at year 2002 for the northern portion shallow zone (4.8 mrem/yr) and was calculated to decrease to $9.5 \times 10^{-11} \mathrm{mrem} / \mathrm{yr}$ in 1,000 years. The estimated dose rate for the northern portion shallow zone in the year 2018 is $2.1 \mathrm{mrem} / \mathrm{yr}$. The overburden dose rate was largest at year $2002(1.8 \mathrm{mrem} / \mathrm{yr})$ and was calculated to decrease to $5.0 \times 10^{-16} \mathrm{mrem} / \mathrm{yr}$ in 1,000 years. The estimated dose rate for the northern portion overburden in the year 2018 is $0.65 \mathrm{mrem} / \mathrm{yr}$. The 2018 date corresponds to the 30-year site cleanup schedule of the Hanford Federal Facility Agreement and Consent Order (Ecology et al. 1998). The dose rate estimates for the northern portion of the site are less than the $15 \mathrm{mrem} / \mathrm{yr}$ RAG.

The confirmatory sampling data from the southern portion of the site was assessed using cleanup verification methodology. After radioactive decay from 2001 to 2007, all RAGs were met for the southern portion of the ash pit, as documented in the calculation briefs for the southern portion (Appendix C). The dose rate calculation for the southern portion of the ash pit after radioactive decay from 2001 to 2007 was $14.3 \mathrm{mrem} / \mathrm{yr}$ (Figure 6). As documented in the RESRAD calculation brief for the southern portion of the site, the dose rate is now calculated to be below the $15 \mathrm{mrem} / \mathrm{yr}$ RAG.

Figure 4. RESRAD Analysis for the Ash Pit Northern Portion Shallow Zone All Radionuclides, All-Pathways Dose Rate Estimate.

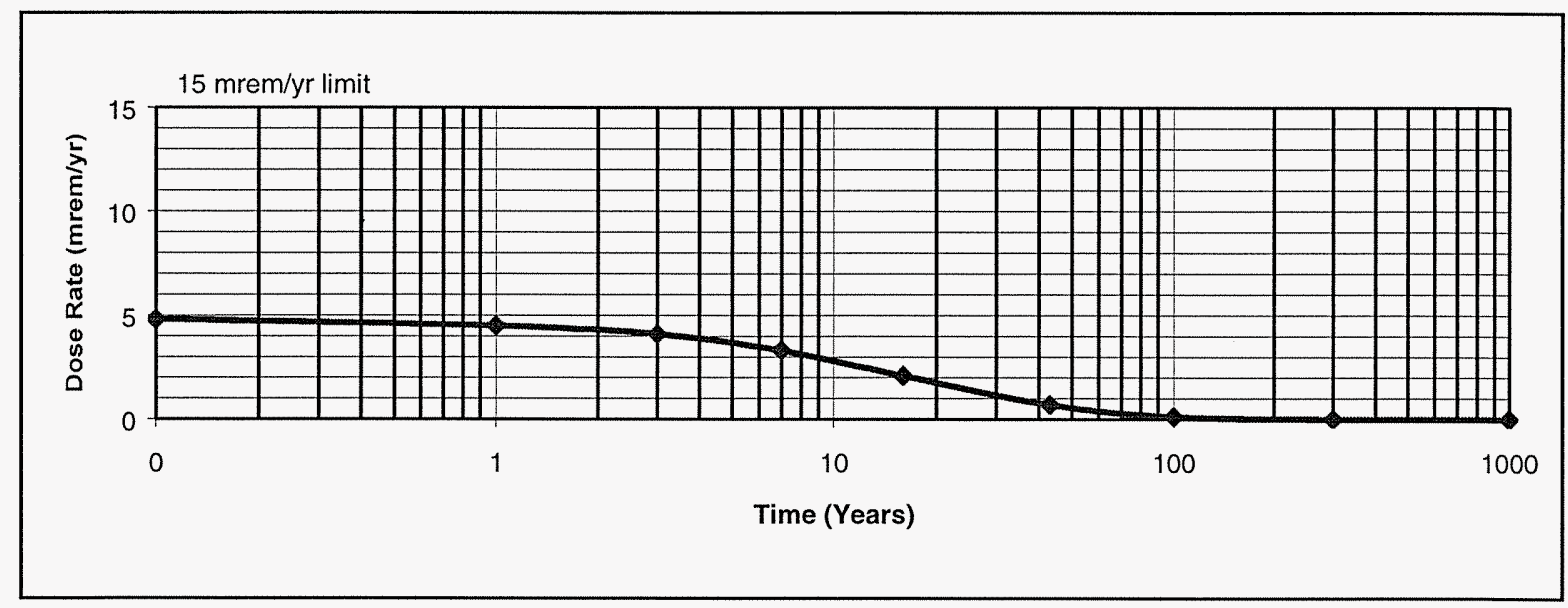


Figure 5. RESRAD Analysis for the Ash Pit Northern Portion Overburden All Radionuclides, All-Pathways Dose Rate Estimate.

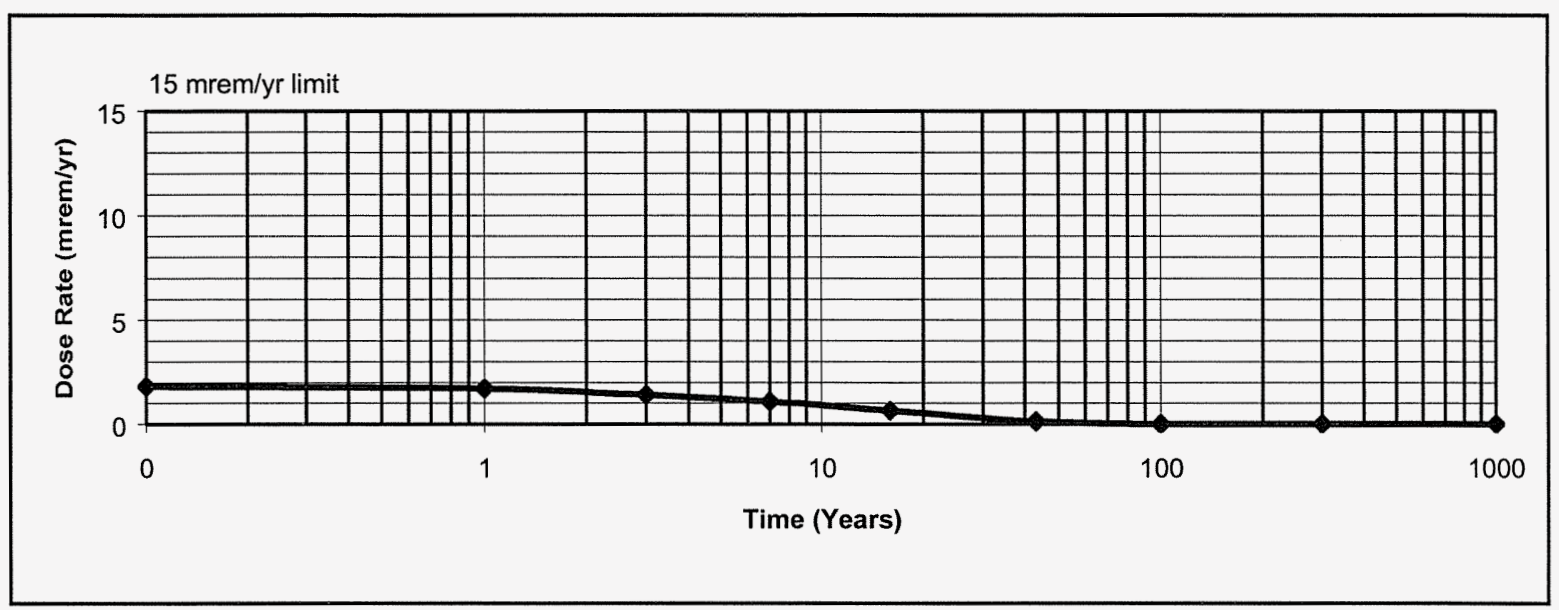

Figure 6. RESRAD Analysis for the Ash Pit Southern Portion Shallow Zone All Radionuclides, All-Pathways Dose Rate Estimate.

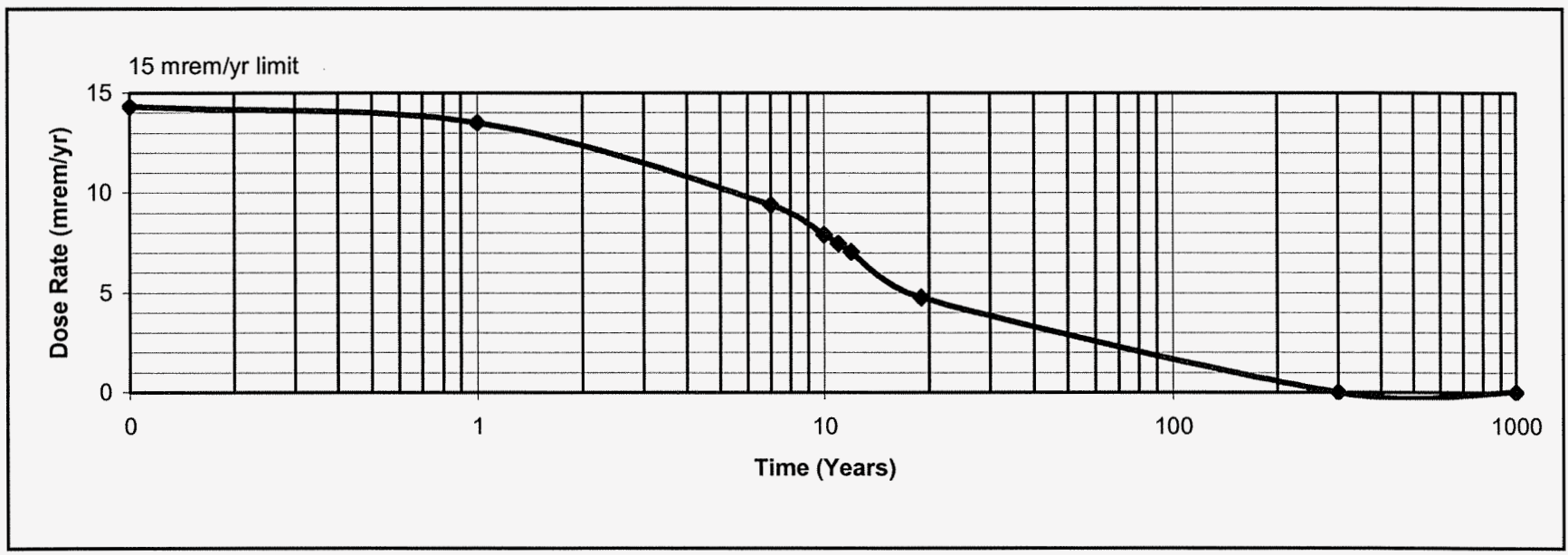

The RESRAD computations for both portions of the site are presented in detail in the referenced calculation briefs summarized in Appendix C.

\subsubsection{Nonradionuclides}

There are no nonradionuclide COCs associated with this site.

\subsection{GROUNDWATER REMEDIAL ACTION GOALS ATTAINED}

\subsubsection{Radionuclides}

The estimated groundwater concentrations for all of the radionuclide COCs contributed by the site soils are shown in the RESRAD calculation brief (Appendix C). Table 3 
shows the total peak concentration predicted for each radionuclide $\mathrm{COC}$ and provides the individual RAGs for comparison. No COC is predicted to exceed the RAGs; therefore, the RAGs are attained for both the northern and southern portions of the site.

\section{Table 3. Estimated Peak Radionuclide Groundwater Concentrations Compared to RAGs.}

\begin{tabular}{|c|c|c|c|}
\hline Radionuclide & $\begin{array}{l}\text { Peak Concentration } \\
\text { (pCi/L) }\end{array}$ & $\begin{array}{l}\text { RAG } \\
(\mathrm{pCi} / \mathrm{L})\end{array}$ & $\begin{array}{c}\text { RAGS Attained? } \\
\text { (Yes/No) }\end{array}$ \\
\hline \multicolumn{4}{|c|}{ Northern Portion } \\
\hline Cesium-137 & 0.00029 & $60^{b}$ & Yes \\
\hline Cobalt-60 & 0.016 & $100^{b}$ & Yes \\
\hline Europium-152 & $\mathrm{a}$ & $200^{b}$ & Yes \\
\hline Europium-154 & $\mathrm{a}$ & $60^{b}$ & Yes \\
\hline Europium-155 & $\mathrm{a}$ & $600^{b}$ & Yes \\
\hline \multicolumn{4}{|c|}{ Overburden } \\
\hline Cesium-137 & a & $60^{b}$ & Yes \\
\hline Cobalt- 60 & $\bar{a}$ & $100^{b}$ & Yes \\
\hline Europium-152 & $\mathrm{a}$ & $200^{b}$ & Yes \\
\hline Europium-154 & $a$ & $60^{b}$ & Yes \\
\hline Europium-155 & $\mathrm{a}$ & $600^{b}$ & Yes \\
\hline \multicolumn{4}{|c|}{ Southern Portion } \\
\hline Cesium-137 & $\mathrm{c}$ & $60^{b}$ & Yes \\
\hline Cobalt-60 & $2.31 \mathrm{E}-03$ & $100^{\mathrm{b}}$ & Yes \\
\hline Europium-152 & $5.48 \mathrm{E}-03$ & $200^{\mathrm{b}}$ & Yes \\
\hline Europium-154 & $4.72 \mathrm{E}-04$ & $60^{b}$ & Yes \\
\hline Europium-155 & $3.08 \mathrm{E}-05$ & $600^{b}$ & Yes \\
\hline
\end{tabular}

${ }^{a}$ These radionuclides do not reach groundwater in 1,000 years.

bookup value corresponding to a dose rate of $4 \mathrm{mrem} / \mathrm{yr}$.

${ }^{c}$ Soil concentrations for these radionuclides are below Hanford Site background and, therefore, were not assessed using RESRAD.

$R A G=$ remedial action goal

RESRAD = RESidual RADioactivity dose assessment model

Individual organ dose rates for beta- and gamma-emitting radionuclides predicted over 1,000 years for the northern and southern portions of the site are shown in Figure 7 , as shown in the comparison to drinking water standards calculation briefs (Appendix C). None of the organ dose rates are predicted to exceed the $4 \mathrm{mrem} / \mathrm{yr}$ standard for 1,000 years following remediation.

There are no alpha emitters identified at this site; therefore, the drinking water standard for nonuranium alpha emitters and the uranium standard of $30 \mu \mathrm{g} / \mathrm{L}$ have been met. Results of the RESRAD calculations are shown in Appendix C. 
Figure 7. Dose Rates to Organs from Groundwater.

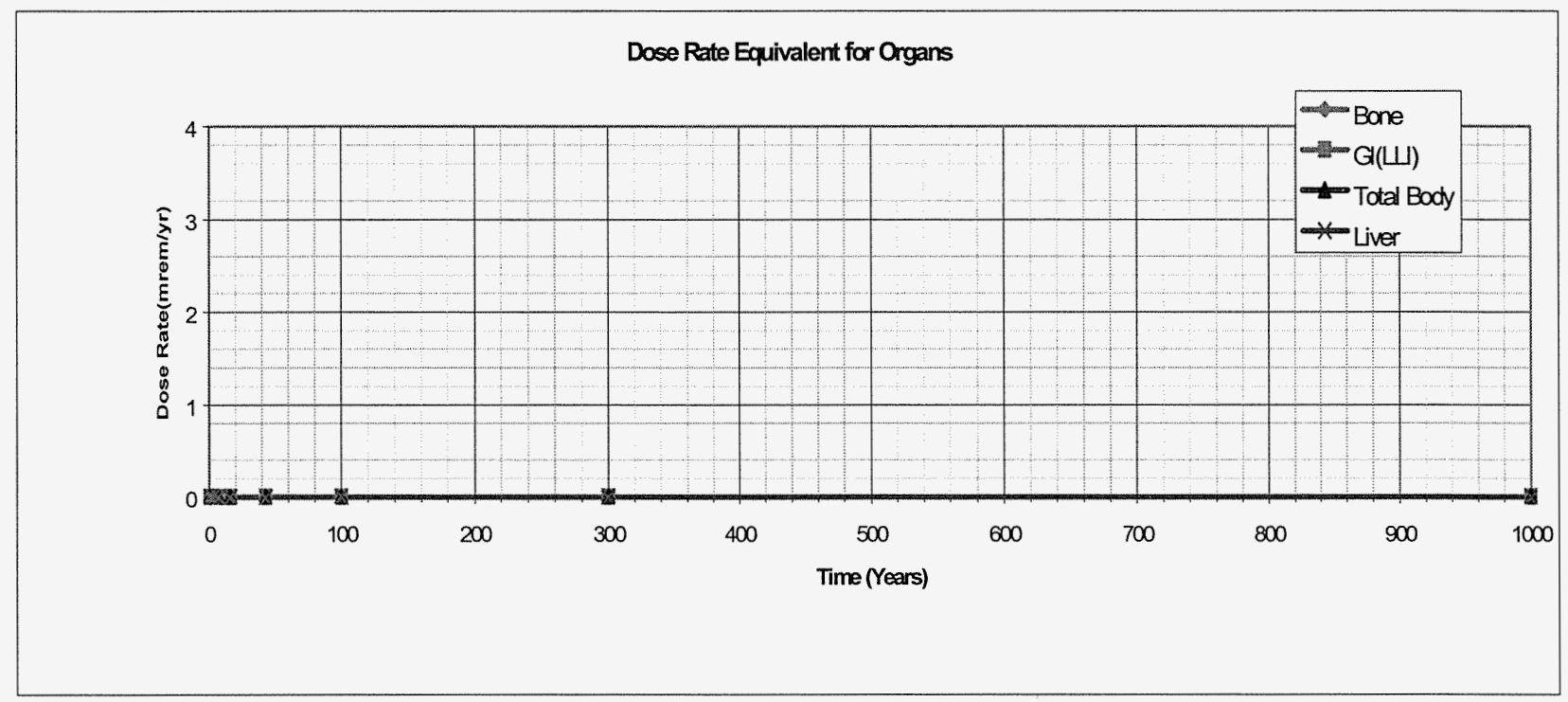

\subsubsection{Nonradionuclides}

There are no nonradionuclide COCs associated with this site.

\subsection{COLUMBIA RIVER REMEDIAL ACTION GOALS ATTAINED}

\subsubsection{Radionuclides}

The river protection RAGs for radionuclides are identical to the groundwater protection RAGs. The RESRAD modeling results for both the northern and southern portion of the site were compared to the groundwater protection RAGs in Table 3 . The results indicated that radionuclides are not predicted to reach groundwater (and, by extension, not predicted to reach the Columbia River) at levels above $4 \mathrm{mrem} / \mathrm{yr}$; therefore, the Columbia River protection RAGs have been attained.

\subsubsection{Nonradionuclides}

There are no nonradionuclide COCs associated with this site.

\subsection{RADIONUCLIDE RISK INFORMATION}

The radionuclide RAG for direct exposure is derived from the ROD (EPA 1997) and is expressed in terms of an allowable radiation dose rate above background (i.e., $15 \mathrm{mrem} / \mathrm{yr}$ ). The RAG evaluation (Section 5.0) involved using the RESRAD model to estimate total annual radiation dose rates for 1,000 years for comparison to the RAG. Radiation presents a carcinogenic risk, and the RESRAD model also calculates the excess lifetime cancer risk associated with the estimated radiation dose rates using the 
EPA's Health Effects Assessment Summary Tables (updated April 16, 2001, "Update of Radionuclide Carcinogenicity Slope Factors," available on the Internet at

Figures 8 and 10 illustrate the excess lifetime cancer risk as estimated using the RESRAD model for the northern portion shallow zone and overburden, respectively.

Figure 12 illustrates excess lifetime cancer risk as estimated using the RESRAD model for the shallow zone of the southern portion of the site. Because of radioactive decay, the risk decreases over time.

For the northern portion shallow zone, the estimated risk was largest, $5.8 \times 10^{-5}$, at the time of the original RESRAD evaluation (year 2002), and decreased to $1.5 \times 10^{-15}$ in 1,000 years. The estimated risk in the year 2018 is $6.8 \times 10^{-5}$. Figure 9 plots the radiation dose rate above background for the northern portion shallow zone, similar to Figure 5, and shows the corresponding radionuclide risk at the time of the original RESRAD evaluation and in the year 2018. For the overburden, the estimated risk is largest, $1.9 \times 10^{-5}$, at the time of the original RESRAD evaluation (year 2002), and decreases to $3.4 \times 10^{-21}$ in 1,000 years. The estimated risk posed by overburden soil in the year 2018 is $7.3 \times 10^{-6}$. Figure 11 plots the radiation dose rate above background for the overburden soil, and shows the corresponding radionuclide risk at present and in the year 2018.

For the southern portion shallow zone, the estimated risk is largest, $2.2 \times 10^{-4}$, at present (year 2007), and decreases to $4.8 \times 10^{-20}$ in 1,000 years. The estimated risk in the year 2018 is $8.4 \times 10^{-4}$. Figure 13 plots the radiation dose rate above background for the southern portion shallow zone, and shows the corresponding radionuclide risk at present and in the year 2018.

Figure 8. RESRAD Analysis for the Northern Portion Shallow Zone Radionuclide Risk, All Pathways

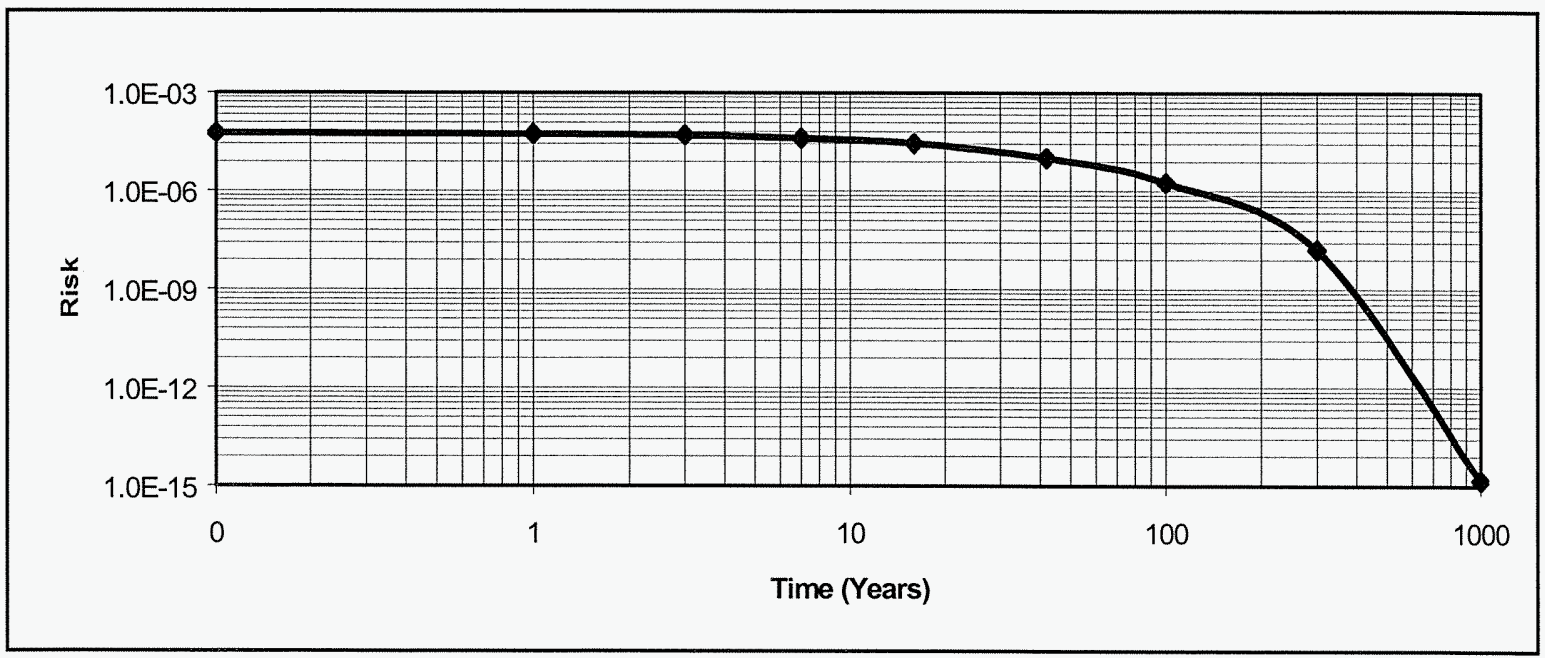


Figure 9. RESRAD Analysis for the Northern Portion Shallow Zone -

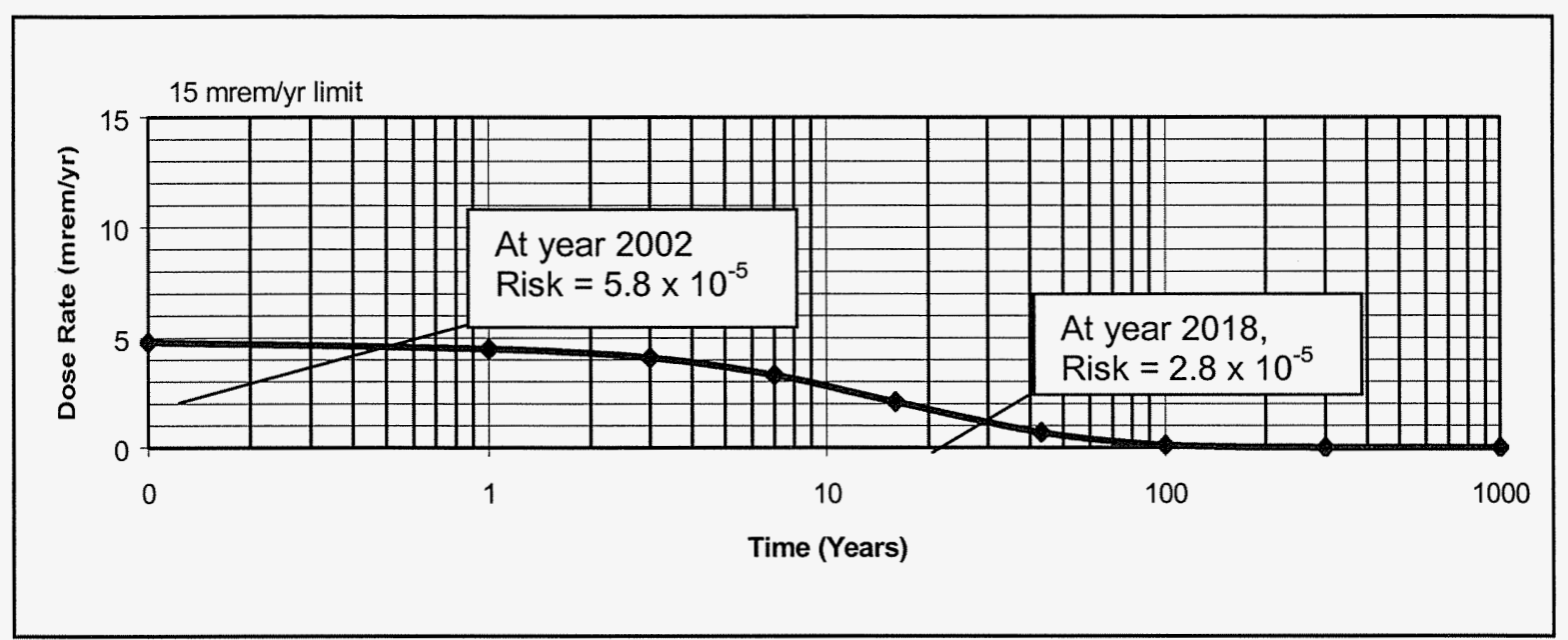

Radionuclide Dose Rate, All Pathways, With Corresponding Risk Values.

Figure 10. RESRAD Analysis for the Northern Portion Overburden - Radionuclide Risk, All Pathways.

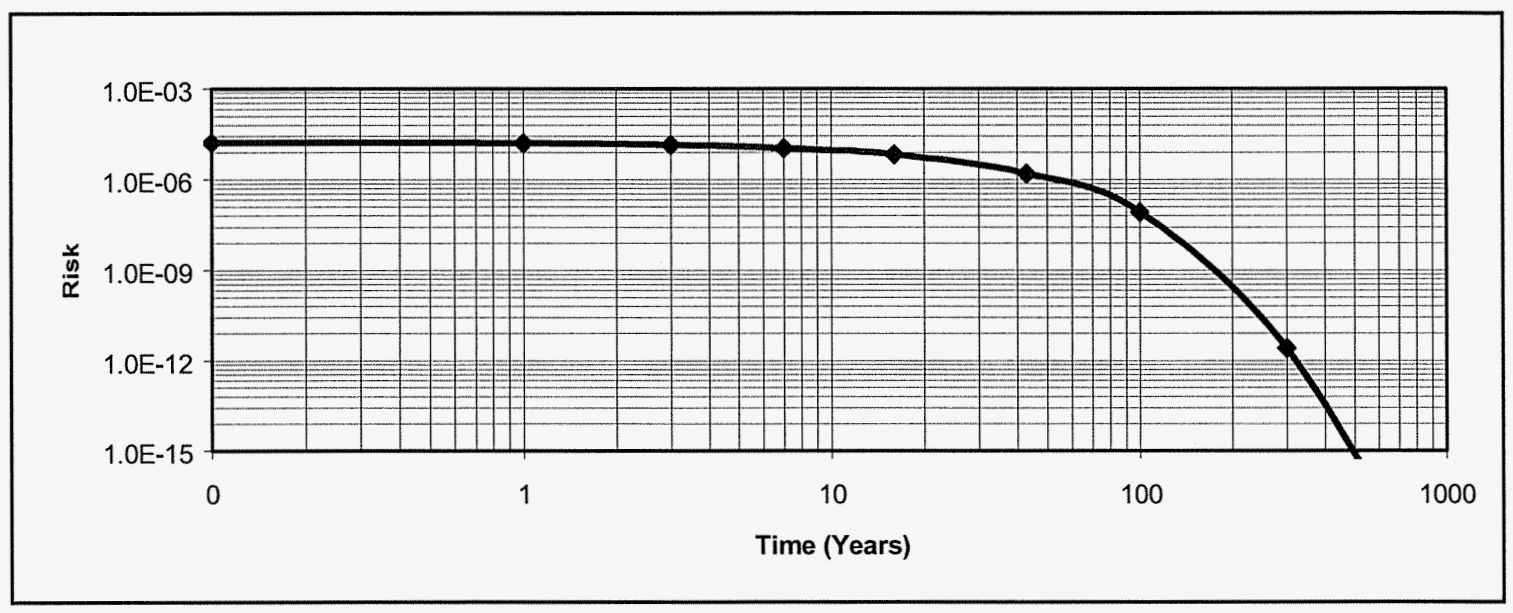


Figure 11. RESRAD Analysis for the Northern Portion Overburden- Radionuclide

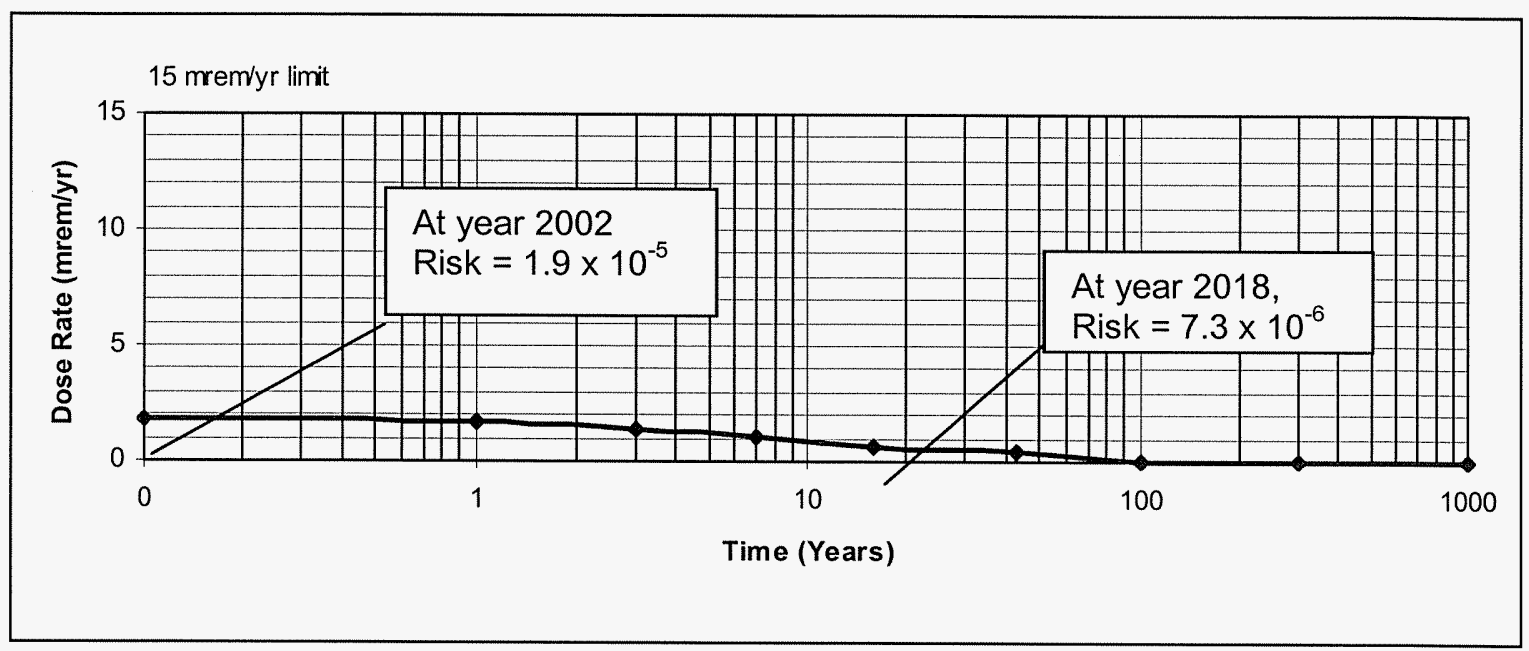

Dose Rate, All Pathways, With Corresponding Risk Values.

Figure 12. RESRAD Analysis for the Southern Portion Shallow Zone Radionuclide Risk, All Pathways.

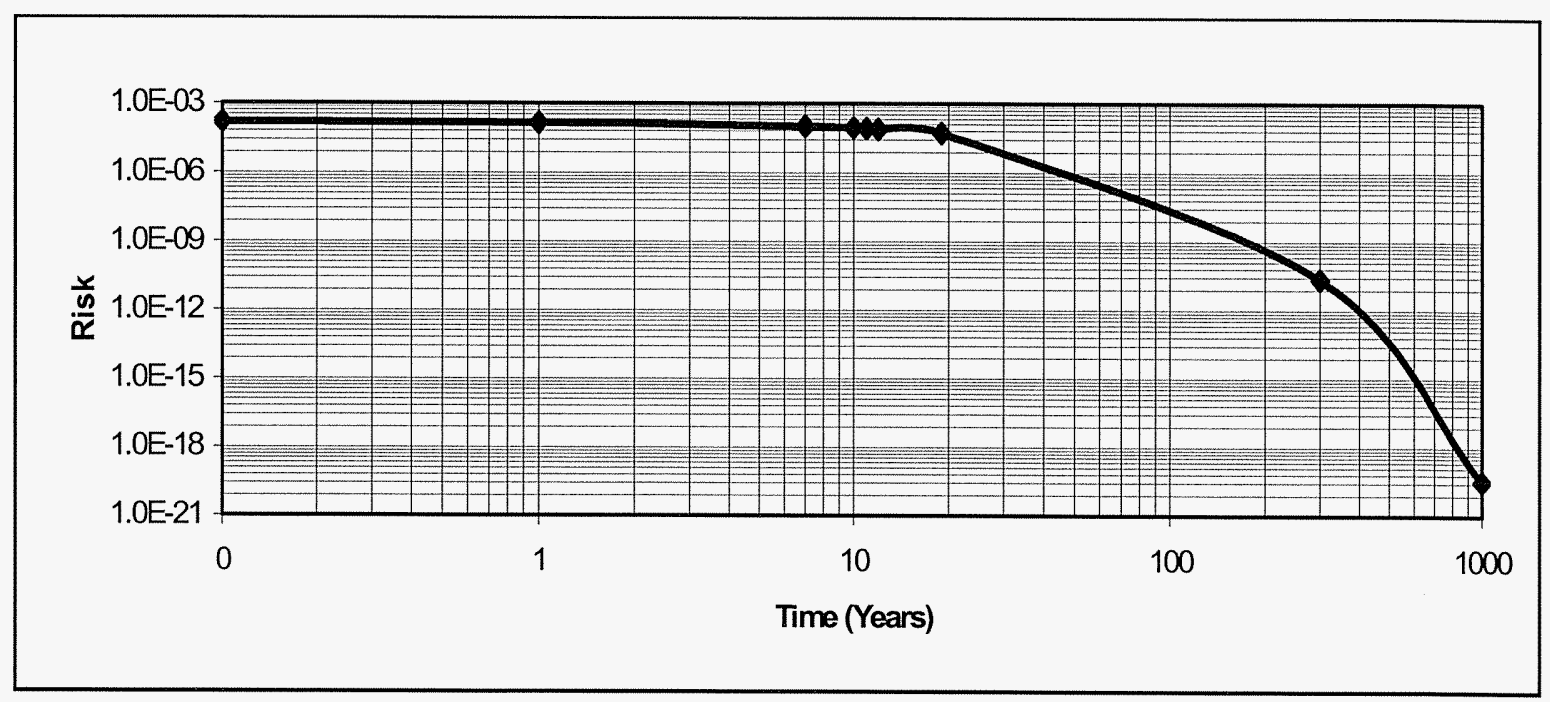


Figure 13. RESRAD Analysis for the Southern Portion Shallow Zone Radionuclide Dose Rate, All Pathways, With Corresponding Risk Values.

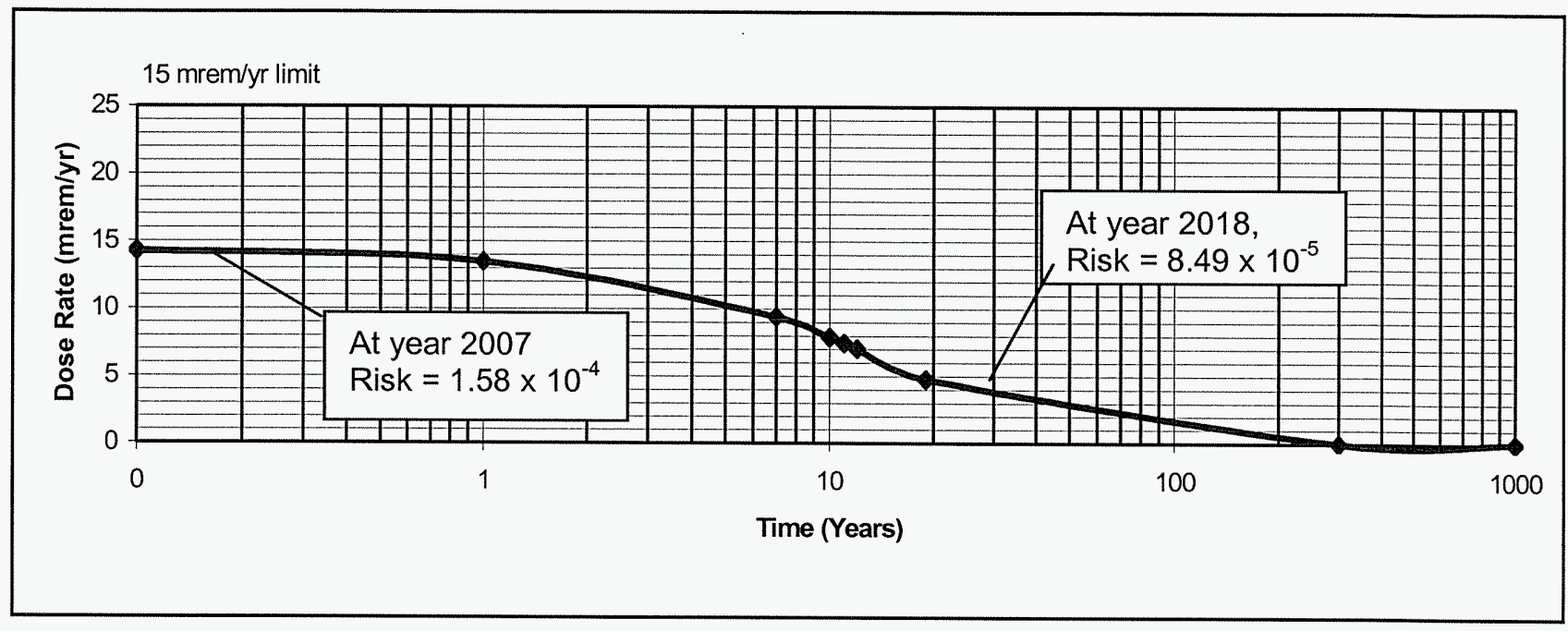

\subsection{STATEMENT OF PROTECTIVENESS}

This CVP demonstrates that remedial action in the northern portion of the 126-F-1 site has achieved the RAOs and corresponding RAGs established in the approved Amendment to the Interim Action ROD (EPA 1997) and RDR/RAWP (DOE-RL 2005). The remaining soils in the northern portion of the 126-F-1 site have been sampled, analyzed, and modeled. The results of this effort indicate that the materials from the $126-\mathrm{F}-1$ site containing COCs at concentrations exceeding RAGs have been excavated and disposed of at ERDF. The northern portion of the 126-F-1 site is verified as remediated in accordance with the ROD (EPA 1997) and has been backfilled.

The confirmatory sampling effort in the southern portion of the 126-F-1 site determined that due to radioactive decay the dose rate would fall below the $15 \mathrm{mrem} / \mathrm{yr}$ RAG in 2007. A current dose evaluation using decayed data determines that the radionuclide dose is $14.3 \mathrm{mrem} / \mathrm{yr}$ above background. These results also indicate that residual concentrations in the shallow zone will support future land uses that can be represented (or bounded) by a rural-residential scenario, and that residual concentrations throughout the site pose no threat to groundwater or the Columbia River.

Remedial actions were not required for the northern and southern portion 126-F-1 site deep zone soils; therefore, institutional controls to prevent uncontrolled drilling or excavation into the deep zone are not required. 


\subsection{REFERENCES}

40 CFR 141, "National Primary Drinking Water Regulations," Code of Federal Regulations, as amended.

40 CFR 141.66, "National Primary Drinking Water Regulations, Maximum Contaminant Levels for Radionuclides," Code of Federal Regulations, as amended.

40 CFR 300, "National Oil and Hazardous Substances Pollution Contingency Plan," Code of Federal Regulations, as amended.

ANL, 2005, RESRAD for Windows, Version 6.3, Argonne National Laboratory, Environmental Assessment Division, Argonne, Illinois.

BHI, 1995, 100-FR-2 Operable Unit Man-Carried Radiological Detection System (MRDS) Radiological Surveys, BHI-00339, Rev. 00, Bechtel Hanford, Inc., Richland, Washington.

BHI, 1996, 126-F-1 Radiological Investigation and Downposting Final Report, BHI00806, Rev. 0, Bechtel Hanford, Inc., Richland, Washington.

BHI, 2001a, Instruction Guide for Remediation of the 100 Areas Waste Sites, 0100X-IG-G0001, Rev. 3, Bechtel Hanford, Inc., Richland, Washington.

$\mathrm{BHI}, 2001 \mathrm{~b}$, Sampling and Analysis Instruction for Confirmatory Sampling of the Southern Portion of the 126-F-1 Ash Pit, BHI-01522, Rev. 1, Bechtel Hanford, Inc., Richland, Washington.

BHI, 2002, Cleanup Verification for the 1607-F2 Septic System, CVP-2002-00005, Rev. 0, Bechtel Hanford, Inc., Richland, Washington.

Blumenkranz, D. B., 1997, Group 4 Field Investigation Results and Remedial Design Recommendations, CCN 051553, interoffice memorandum to K. E. Cook, dated October 8, 1997, Bechtel Hanford, Inc., Richland, Washington.

DOE Order 5400.5, Radiation Protection of the Public and the Environment, U.S. Department of Energy, Washington, D.C.

DOE-RL, 1996, Hanford Site Background: Part 2, Soil Background for Radionuclides, DOE/RL-96-12, Rev. 0, U.S. Department of Energy, Richland Operations Office, Richland, Washington.

DOE-RL, 1997, Data Quality Objectives Summary Report for the 100-FR-I, 100-FR-2, 100-HR-1, 100-KR-1, and 100-KR-2 Group 4 Waste Sites, DOE/RL-97-61, Rev. 0, U.S. Department of Energy, Richland Operations Office, Richland, Washington. 
DOE-RL, 2000, Sampling and Analysis Plan for the 100 Area Remaining Sites, DOE/RL-99-58, Rev. 0, U.S. Department of Energy, Richland Operations Office, Richland, Washington.

DOE-RL, 2001, 100 Area Remedial Action Sampling and Analysis Plan, DOE/RL-96-22, Rev. 3, U.S. Department of Energy, Richland Operations Office, Richland, Washington.

DOE-RL, 2005, Remedial Design Report/Remedial Action Work Plan for the 100 Area, DOE/RL-96-17, Rev. 5, U.S. Department of Energy, Richland Operations Office, Richland, Washington.

DOE-RL, 2007, Tri-Party Agreement Handbook Management Procedures, RL-TPA-900001, Guideline Number TPA-MP-14, Rev. 1, "Maintenance of the Waste Information Data System (WIDS)," U.S. Department of Energy, Richland Operations Office, Richland, Washington.

Ecology, EPA, and DOE, 1998, Hanford Federal Facility Agreement and Consent Order, 2 vols., as amended, Washington State Department of Ecology, U.S. Environmental Protection Agency, and U.S. Department of Energy, Olympia, Washington.

EPA, 1996, Guidance for Data Quality Assessment, EPA QA/G-9, U.S. Environmental Protection Agency, Office of Research and Development, Washington, D.C.

EPA, 1997, Amendment to the Interim Action Record of Decision for the 100-BC-1, 100-DR-1, and 100-HR-1 Operable Units, U.S. Environmental Protection Agency, Region 10, Seattle, Washington.

EPA, 1999, Interim Action Record of Decision for the 100-BC-1, 100-BC-2, 100-DR-1, 100-DR-2, 100-FR-1, 100-FR-2, 100-HR-1, 100-HR-2, 100-KR-1, 100-KR-2, 100-IU-2, 100-IU-6, and 200-CW-3 Operable Units, Hanford Site, Benton County, Washington, July 1999, U.S. Environmental Protection Agency, Region 10, Seattle, Washington.

WAC 173-340-740(7)(g), "Model Toxics Control Act - Cleanup, Soil Cleanup Standards," Washington Administrative Code, as amended, 1996. 
APPENDIX A

SUMMARY OF VERIFICATION SOIL SAMPLING AND ANALYTICAL RESULTS 
CVP-2002-00004

Rev. 1

A-ii 
Table A-1. Northern Portion Shallow Zone Cleanup Verification Data (Sample Date 7/16/02 and 7/17/02). (2 pages)

\begin{tabular}{|c|c|c|c|c|c|c|c|c|c|c|c|c|c|c|c|c|c|}
\hline \multirow{2}{*}{$\begin{array}{l}\text { Sampling } \\
\text { Area }\end{array}$} & \multirow{2}{*}{$\begin{array}{c}\text { HEIS } \\
\text { Number }\end{array}$} & \multirow{2}{*}{ Sample Date } & \multicolumn{3}{|c|}{ Cs-137 } & \multicolumn{3}{|c|}{ Co-60 } & \multicolumn{3}{|c|}{ Eu-152 } & \multicolumn{3}{|c|}{ Eu-154 } & \multicolumn{3}{|c|}{ Eu-155 } \\
\hline & & & $\mathrm{pCi} / \mathrm{g}$ & $\mathbf{Q}$ & MDA & $\mathrm{pCi} / \mathrm{g}$ & $\mathbf{Q}$ & MDA & $\mathrm{pCi} / \mathrm{g}$ & $\mathbf{Q}$ & MDA & $\mathrm{pCi} / \mathrm{g}$ & $\mathbf{Q}$ & MDA & $\mathrm{pCi} / \mathrm{g}$ & $\mathbf{Q}$ & MDA \\
\hline $\mathrm{A} 1$ & $\mathrm{~J} 000 \mathrm{C} 2$ & $07 / 16 / 2002$ & $9.30 \mathrm{E}-02$ & $a$ & $3.50 \mathrm{E}-02$ & $1.10 \mathrm{E}-01$ & $U$ & $1.10 \mathrm{E}-01$ & $1.80 \mathrm{E}-01$ & $U$ & $1.80 \mathrm{E}-01$ & $1.20 \mathrm{E}-01$ & $U$ & $1.20 \mathrm{E}-01$ & 1.10E-01 & $\mathrm{U}$ & 1.10E-01 \\
\hline $\begin{array}{l}\text { Duplicate of } \\
\text { J000C2 }\end{array}$ & J00OF0 & $07 / 16 / 2002$ & 7.00E-02 & $a$ & 4.50E-02 & 4.20E-02 & $U$ & 4.20E-02 & 1.10E-01 & $U$ & $1.10 \mathrm{E}-01$ & $1.40 \mathrm{E}-01$ & $U$ & $1.40 \mathrm{E}-01$ & $1.00 \mathrm{E}-01$ & $\mathrm{U}$ & $1.00 \mathrm{E}-01$ \\
\hline $\begin{array}{l}\text { Split of } \\
\text { J000C2 }\end{array}$ & J000JO & $7 / 16 / 2002$ & 5.91E-02 & & 1.79E-02 & $6.25 E-03$ & $U$ & $2.18 E-02$ & 7.38E-02 & $U$ & $5.48 \mathrm{E}-02$ & $1.57 \mathrm{E}-02$ & $U$ & $6.86 \mathrm{E}-02$ & $4.99 \mathrm{E}-02$ & $\mathrm{U}$ & 4.88E-02 \\
\hline E9 & J000F1 & $07 / 16 / 2002$ & $2.88 \mathrm{E}-01$ & & $5.20 \mathrm{E}-02$ & $6.80 \mathrm{E}-02$ & $U$ & $6.80 \mathrm{E}-02$ & $9.54 \mathrm{E}-01$ & & $1.30 \mathrm{E}-01$ & $2.10 E-01$ & $U$ & $2.10 E-01$ & $1.60 \mathrm{E}-01$ & $U$ & $1.60 \mathrm{E}-01$ \\
\hline $\begin{array}{l}\text { Duplicate of } \\
\text { J000F1 }\end{array}$ & J00OH9 & 07/16/2002 & $3.73 E-01$ & & $6.10 \mathrm{E}-02$ & 5.70E-02 & & 4.10E-02 & 9.97E-01 & & $1.10 \mathrm{E}-01$ & $1.70 \mathrm{E}-01$ & $U$ & $1.70 \mathrm{E}-01$ & $1.30 \mathrm{E}-01$ & $U$ & 1.30E-01 \\
\hline $\begin{array}{l}\text { Split of } \\
\text { J000F1 }\end{array}$ & J000J1 & $07 / 16 / 2002$ & 4.56E-01 & & 2.91E-02 & $3.58 \mathrm{E}-02$ & $U$ & $3.13 \mathrm{E}-02$ & $1.06 E+00$ & & $6.90 \mathrm{E}-02$ & $1.48 \mathrm{E}-01$ & U & $1.01 \mathrm{E}-01$ & $9.10 \mathrm{E}-02$ & $U$ & 7.51E-02 \\
\hline $\mathrm{A} 2$ & J000C3 & $07 / 16 / 2002$ & 5.33E-01 & & $3.60 E-02$ & $3.50 \mathrm{E}-02$ & $a$ & $3.10 \mathrm{E}-02$ & $4.88 \mathrm{E}-01$ & & $6.70 \mathrm{E}-02$ & $1.00 \mathrm{E}-01$ & $U$ & $1.00 \mathrm{E}-01$ & $6.30 \mathrm{E}-02$ & $U$ & 6.30E-02 \\
\hline A3 & $\mathrm{J} 000 \mathrm{C} 4$ & $07 / 16 / 2002$ & $1.12 E+00$ & & $4.00 \mathrm{E}-02$ & $7.60 \mathrm{E}-02$ & & $3.00 \mathrm{E}-02$ & $4.66 \mathrm{E}-01$ & & $7.30 \mathrm{E}-02$ & 1.20E-01 & $U$ & $1.20 \mathrm{E}-01$ & $6.70 \mathrm{E}-02$ & $\bar{U}$ & $6.70 \mathrm{E}-02$ \\
\hline A4 & J000C5 & $07 / 16 / 2002$ & $5.13 \mathrm{E}-01$ & & $4.70 \mathrm{E}-02$ & $9.50 \mathrm{E}-02$ & $U$ & $9.50 \mathrm{E}-02$ & 4.35E-01 & & $1.20 \mathrm{E}-01$ & 1.20E-01 & $U$ & 1.20E-01 & $1.80 \mathrm{E}-01$ & $U$ & 1.80E-01 \\
\hline B5 & J000C6 & $07 / 16 / 2002$ & 2.09E-01 & & $4.40 \mathrm{E}-02$ & 4.10E-02 & $U$ & $4.10 \mathrm{E}-02$ & $1.60 \mathrm{E}-01$ & $U$ & 1.60E-01 & $1.20 \mathrm{E}-01$ & $U$ & $1.20 \mathrm{E}-01$ & 1.10E-01 & $U$ & $1.10 \mathrm{E}-01$ \\
\hline B6 & J000C7 & $07 / 16 / 2002$ & $1.50 \mathrm{E}-01$ & & $3.10 \mathrm{E}-02$ & $5.90 \mathrm{E}-02$ & $U$ & $5.90 \mathrm{E}-02$ & $2.14 \mathrm{E}-01$ & & $5.90 \mathrm{E}-02$ & $1.00 \mathrm{E}-01$ & $U$ & 1.00E-01 & 1.00E-01 & $U$ & $1.00 \mathrm{E}-01$ \\
\hline B7 & J000C8 & $07 / 16 / 2002$ & $3.09 \mathrm{E}-01$ & & $6.60 \mathrm{E}-02$ & $7.00 \mathrm{E}-02$ & $\mathrm{U}$ & $7.00 \mathrm{E}-02$ & $4.46 \mathrm{E}-01$ & & $1.10 \mathrm{E}-01$ & $1.90 \mathrm{E}-01$ & $U$ & $1.90 \mathrm{E}-01$ & $9.80 \mathrm{E}-02$ & $U$ & 9.80 E-02 \\
\hline B8 & J000C9 & $07 / 16 / 2002$ & $1.94 \mathrm{E}-01$ & & $5.30 \mathrm{E}-02$ & $6.20 \mathrm{E}-02$ & $U$ & $6.20 \mathrm{E}-02$ & $6.30 \mathrm{E}-01$ & & $9.20 \mathrm{E}-02$ & $1.60 \mathrm{E}-01$ & $U$ & $1.60 \mathrm{E}-01$ & $1.10 \mathrm{E}-01$ & $U$ & $1.10 \mathrm{E}-01$ \\
\hline $\mathrm{Cg}$ & J000D0 & $07 / 16 / 2002$ & $2.56 \mathrm{E}-01$ & & $3.60 \mathrm{E}-02$ & $5.00 \mathrm{E}-02$ & & $2.80 \mathrm{E}-02$ & $7.78 \mathrm{E}-01$ & & $5.80 \mathrm{E}-02$ & $1.10 \mathrm{E}-01$ & $\mathrm{U}$ & 1.10E-01 & $9.10 \mathrm{E}-02$ & $\bar{U}$ & $9.10 E-02$ \\
\hline $\mathrm{C} 10$ & J000D1 & $07 / 16 / 2002$ & $6.15 E-01$ & & $7.10 \mathrm{E}-02$ & $4.80 \mathrm{E}-02$ & $U$ & $4.80 \mathrm{E}-02$ & 7.70E-01 & & $1.70 \mathrm{E}-01$ & 1.30E-01 & $U$ & 1.30E-01 & $2.40 \mathrm{E}-01$ & $U$ & $2.40 E-01$ \\
\hline $\mathrm{C1}$ & J000D2 & $07 / 16 / 2002$ & $2.10 \mathrm{E}-01$ & & $9.00 \mathrm{E}-02$ & $7.60 \mathrm{E}-02$ & $U$ & $7.60 \mathrm{E}-02$ & $4.34 \mathrm{E}-01$ & & $1.60 \mathrm{E}-01$ & $2.00 \mathrm{E}-01$ & $U$ & $2.00 \mathrm{E}-01$ & $1.70 \mathrm{E}-01$ & $U$ & $1.70 \mathrm{E}-01$ \\
\hline $\mathrm{C} 2$ & J000D3 & $07 / 16 / 2002$ & 1.36E-01 & & $4.70 \mathrm{E}-02$ & $4.40 \mathrm{E}-02$ & $U$ & $4.40 \mathrm{E}-02$ & $2.40 E-01$ & $U$ & $2.40 \mathrm{E}-01$ & $1.20 \mathrm{E}-01$ & $\mathrm{U}$ & $1.20 \mathrm{E}-01$ & $1.00 \mathrm{E}-01$ & $U$ & $1.00 \mathrm{E}-01$ \\
\hline D3 & J000D4 & $07 / 16 / 2002$ & 1.96E-01 & & $5.10 \mathrm{E}-02$ & $4.10 \mathrm{E}-02$ & $U$ & $4.10 \mathrm{E}-02$ & 4.30E-01 & $U$ & $4.30 \mathrm{E}-01$ & 1.30E-01 & $U$ & 1.30E-01 & $8.90 \mathrm{E}-02$ & $U$ & $8.90 E-02$ \\
\hline D4 & J000D5 & $07 / 16 / 2002$ & $3.42 \mathrm{E}-01$ & & $6.00 \mathrm{E}-02$ & $5.10 \mathrm{E}-02$ & $U$ & $5.10 \mathrm{E}-02$ & $4.00 \mathrm{E}-01$ & $U$ & $4.00 \mathrm{E}-01$ & $1.60 \mathrm{E}-01$ & $U$ & $1.60 \mathrm{E}-01$ & $1.80 \mathrm{E}-01$ & $U$ & $1.80 \mathrm{E}-01$ \\
\hline D5 & J000D6 & $07 / 16 / 2002$ & 4.30E-02 & $a$ & $3.50 \mathrm{E}-02$ & $3.50 \mathrm{E}-02$ & $U$ & $3.50 \mathrm{E}-02$ & $1.50 \mathrm{E}-01$ & $U$ & $1.50 \mathrm{E}-01$ & 1.10E-01 & $\mathrm{U}$ & $1.10 \mathrm{E}-01$ & $9.30 \mathrm{E}-02$ & $U$ & $9.30 \mathrm{E}-02$ \\
\hline D6 & J000D7 & $07 / 16 / 2002$ & 1.75E-01 & & 4.30E-02 & $4.20 \mathrm{E}-02$ & $U$ & $4.20 \mathrm{E}-02$ & $2.75 E-01$ & & $8.30 \mathrm{E}-02$ & 1.30E-01 & $U$ & 1.30E-01 & $1.00 E-01$ & $U$ & $1.00 \mathrm{E}-01$ \\
\hline E7 & J000D8 & $07 / 16 / 2002$ & $8.31 \mathrm{E}-01$ & & $6.20 \mathrm{E}-02$ & 1.10E-01 & $U$ & $1.10 \mathrm{E}-01$ & $1.67 \mathrm{E}+00$ & & $1.00 \mathrm{E}-01$ & $3.00 \mathrm{E}-01$ & $U$ & $3.00 \mathrm{E}-01$ & $1.70 \mathrm{E}-01$ & $U$ & $1.70 \mathrm{E}-01$ \\
\hline E8 & J000D9 & $07 / 16 / 2002$ & $4.42 E-01$ & & $6.80 \mathrm{E}-02$ & $6.60 \mathrm{E}-02$ & $U$ & $6.60 \mathrm{E}-02$ & $6.77 E-01$ & & $1.20 \mathrm{E}-01$ & $2.00 E-01$ & $u$ & $2.00 \mathrm{E}-01$ & $1.90 \mathrm{E}-01$ & $U$ & $1.90 E-01$ \\
\hline E10 & J000F2 & $07 / 17 / 2002$ & $1.09 E+00$ & & $2.40 \mathrm{E}-02$ & $7.10 \mathrm{E}-02$ & & $1.70 \mathrm{E}-02$ & $1.93 E+00$ & & $5.80 \mathrm{E}-02$ & 1.60E-01 & & $5.50 \mathrm{E}-02$ & $9.40 \mathrm{E}-02$ & $U$ & $9.40 \mathrm{E}-02$ \\
\hline $\mathrm{F} 1$ & J000F3 & $07 / 17 / 2002$ & 7.13E-01 & & $3.60 \mathrm{E}-02$ & $1.00 \mathrm{E}-01$ & & $2.70 \mathrm{E}-02$ & $2.70 E+00$ & & $6.00 E-02$ & $2.47 E-01$ & & $8.60 \mathrm{E}-02$ & $9.40 \mathrm{E}-02$ & $U$ & $9.40 \mathrm{E}-02$ \\
\hline $\mathrm{F} 2$ & J000F4 & $07 / 17 / 2002$ & $3.96 \mathrm{E}-01$ & & $1.10 \mathrm{E}-02$ & $2.70 \mathrm{E}-02$ & $a$ & 1.40E-02 & $4.42 E-01$ & & $3.00 \mathrm{E}-02$ & $6.00 \mathrm{E}-02$ & $U$ & $6.00 \mathrm{E}-02$ & $3.20 \mathrm{E}-02$ & $a$ & $3.20 \mathrm{E}-02$ \\
\hline $\mathrm{F} 3$ & J000F5 & $07 / 17 / 2002$ & 9.30E-02 & $a$ & $5.20 \mathrm{E}-02$ & $5.40 \mathrm{E}-02$ & $U$ & $5.40 \mathrm{E}-02$ & 3.07E-01 & & $9.20 \mathrm{E}-02$ & $1.80 \mathrm{E}-01$ & $U$ & $1.80 \mathrm{E}-01$ & $1.20 \mathrm{E}-01$ & $U$ & 1.20E-01 \\
\hline $\mathrm{F} 4$ & J000F6 & $07 / 17 / 2002$ & $1.10 \mathrm{E}-01$ & $U$ & $1.10 \mathrm{E}-01$ & $4.80 \mathrm{E}-02$ & $U$ & $4.80 \mathrm{E}-02$ & 1.60E-01 & $U$ & $1.60 E-01$ & $1.50 \mathrm{E}-01$ & $U$ & 1.50E-01 & $1.10 \mathrm{E}-01$ & $U$ & 1.10E-01 \\
\hline G5 & J000F7 & $07 / 17 / 2002$ & $1.22 \mathrm{E}-01$ & & 1.70E-02 & $2.30 \mathrm{E}-02$ & $U$ & $2.30 \mathrm{E}-02$ & $2.20 \mathrm{E}-01$ & & $3.40 \mathrm{E}-02$ & $4.90 \mathrm{E}-02$ & $U$ & $4.90 \mathrm{E}-02$ & $3.50 \mathrm{E}-02$ & $U$ & 3.50E-02 \\
\hline
\end{tabular}


Table A-1. Northern Portion Shallow Zone Cleanup Verification Data (Sample Date 7/16/02 and 7/17/02). (2 pages)

\begin{tabular}{|c|c|c|c|c|c|c|c|c|c|c|c|c|c|c|c|c|c|}
\hline \multirow{2}{*}{$\begin{array}{c}\text { Sampling } \\
\text { Area }\end{array}$} & \multirow{2}{*}{$\begin{array}{c}\text { HEIS } \\
\text { Number }\end{array}$} & \multirow{2}{*}{ Sample Date } & \multicolumn{3}{|c|}{ Cs-137 } & \multicolumn{3}{|c|}{ Co-60 } & \multicolumn{3}{|c|}{ Eu-152 } & \multicolumn{3}{|c|}{ Eu-154 } & \multicolumn{3}{|c|}{ Eu-155 } \\
\hline & & & $\mathrm{pCi} / \mathrm{g}$ & $\mathbf{Q}$ & MDA & $\mathrm{pCi} / \mathrm{g}$ & $\mathbf{Q}$ & MDA & $\mathrm{pCi} / \mathrm{g}$ & $\mathbf{Q}$ & MDA & $\mathrm{pCi} / \mathrm{g}$ & $\mathbf{Q}$ & MDA & $\mathrm{pCi} / \mathrm{g}$ & $\mathbf{Q}$ & MDA \\
\hline G6 & J000F8 & $07 / 17 / 2002$ & $7.30 \mathrm{E}-02$ & & $3.00 \mathrm{E}-02$ & $2.80 \mathrm{E}-02$ & $U$ & $2.80 \mathrm{E}-02$ & $1.40 \mathrm{E}-01$ & $U$ & $1.40 \mathrm{E}-01$ & $9.40 \mathrm{E}-02$ & $U$ & $9.40 \mathrm{E}-02$ & $5.50 \mathrm{E}-02$ & $\mathrm{U}$ & $5.50 \mathrm{E}-02$ \\
\hline G7 & J000F9 & $07 / 17 / 2002$ & $9.30 \mathrm{E}-02$ & $a$ & $4.70 \mathrm{E}-02$ & $4.60 \mathrm{E}-02$ & $U$ & $4.60 \mathrm{E}-02$ & $1.60 \mathrm{E}-01$ & $U$ & $1.60 \mathrm{E}-01$ & $1.60 \mathrm{E}-01$ & $U$ & $1.60 \mathrm{E}-01$ & $1.00 \mathrm{E}-01$ & $U$ & $1.00 \mathrm{E}-01$ \\
\hline G8 & $\mathrm{JOOOHO}$ & $07 / 17 / 2002$ & $9.20 \mathrm{E}-02$ & $U$ & $9.20 \mathrm{E}-02$ & $4.90 \mathrm{E}-02$ & $\mathrm{U}$ & $4.90 \mathrm{E}-02$ & $1.10 \mathrm{E}-01$ & $U$ & $1.10 \mathrm{E}-01$ & $1.60 \mathrm{E}-01$ & $U$ & $1.60 \mathrm{E}-01$ & $7.50 \mathrm{E}-02$ & $U$ & $7.50 \mathrm{E}-02$ \\
\hline $\mathrm{H} 9$ & $\mathrm{~J} 000 \mathrm{H} 1$ & $07 / 17 / 2002$ & $2.08 \mathrm{E}-01$ & & $4.10 \mathrm{E}-02$ & $6.70 \mathrm{E}-02$ & $U$ & $6.70 \mathrm{E}-02$ & $6.14 \mathrm{E}-01$ & & $8.10 \mathrm{E}-02$ & $1.20 \mathrm{E}-01$ & $U$ & $1.20 \mathrm{E}-01$ & $1.20 \mathrm{E}-01$ & $U$ & $1.20 \mathrm{E}-01$ \\
\hline $\mathrm{H} 10$ & $\mathrm{~J} 000 \mathrm{H} 2$ & $07 / 17 / 2002$ & $2.86 \mathrm{E}-01$ & & $4.50 \mathrm{E}-02$ & $6.50 \mathrm{E}-02$ & & $3.70 \mathrm{E}-02$ & $6.23 \mathrm{E}-01$ & & $7.70 \mathrm{E}-02$ & $1.40 \mathrm{E}-01$ & $U$ & $1.40 \mathrm{E}-01$ & $1.30 \mathrm{E}-01$ & $\mathrm{U}$ & 1.30E-01 \\
\hline $\mathrm{H} 1$ & $\mathrm{~J} 00 \mathrm{OH} 3$ & $07 / 17 / 2002$ & $5.44 \mathrm{E}-01$ & & $3.70 \mathrm{E}-02$ & $6.80 \mathrm{E}-02$ & $U$ & $6.80 \mathrm{E}-02$ & $5.84 \mathrm{E}-01$ & & $7.40 \mathrm{E}-02$ & $1.20 \mathrm{E}-01$ & $\mathrm{U}$ & $1.20 \mathrm{E}-01$ & $8.70 \mathrm{E}-02$ & U & $8.70 \mathrm{E}-02$ \\
\hline $\mathrm{H} 2$ & $\mathrm{~J} 000 \mathrm{H} 4$ & $07 / 17 / 2002$ & $5.90 \mathrm{E}-02$ & $U$ & $5.90 \mathrm{E}-02$ & $5.60 \mathrm{E}-02$ & $U$ & $5.60 \mathrm{E}-02$ & 1.30E-01 & U & $1.30 \mathrm{E}-01$ & $2.20 \mathrm{E}-01$ & $U$ & $2.20 \mathrm{E}-01$ & $1.00 \mathrm{E}-01$ & $\mathrm{U}$ & $1.00 \mathrm{E}-01$ \\
\hline 13 & $\mathrm{~J} 00 \mathrm{OH} 5$ & $07 / 17 / 2002$ & 1.00E-01 & & $4.30 \mathrm{E}-01$ & $6.20 \mathrm{E}-02$ & $U$ & $6.20 \mathrm{E}-02$ & $3.91 \mathrm{E}-01$ & & $8.50 \mathrm{E}-02$ & 1.40E-01 & $\mathrm{U}$ & $1.40 \mathrm{E}-01$ & $1.30 \mathrm{E}-01$ & $u$ & $1.30 \mathrm{E}-01$ \\
\hline 14 & $\mathrm{~J} 000 \mathrm{H} 6$ & $07 / 17 / 2002$ & $1.09 \mathrm{E}-01$ & & $4.10 \mathrm{E}-02$ & $3.70 \mathrm{E}-02$ & & $3.70 \mathrm{E}-02$ & 1.10E-01 & U & $1.10 \mathrm{E}-01$ & $1.30 \mathrm{E}-01$ & $\mathrm{U}$ & $1.30 \mathrm{E}-01$ & $7.30 \mathrm{E}-02$ & $U$ & $7.30 \mathrm{E}-02$ \\
\hline 15 & $\mathrm{~J} 000 \mathrm{H} 7$ & $07 / 17 / 2002$ & 3.11E-01 & & $5.90 \mathrm{E}-02$ & $6.70 \mathrm{E}-02$ & U & $6.70 \mathrm{E}-02$ & $2.00 \mathrm{E}-01$ & U & $2.00 \mathrm{E}-01$ & $1.90 \mathrm{E}-01$ & $U$ & 1.90E-01 & $9.40 \mathrm{E}-02$ & $U$ & $9.40 \mathrm{E}-02$ \\
\hline 16 & $\mathrm{~J} 000 \mathrm{H} 8$ & $07 / 17 / 2002$ & $3.14 \mathrm{E}-01$ & & $3.10 \mathrm{E}-02$ & 1.10E-01 & $U$ & 1.10E-01 & 7.84E-01 & & $8.80 \mathrm{E}-02$ & $1.70 \mathrm{E}-01$ & $\mathrm{U}$ & $1.70 \mathrm{E}-01$ & $1.30 \mathrm{E}-01$ & $U$ & 1.30E-01 \\
\hline
\end{tabular}

"Because of laboratory reporting conditions, these data were given a non-relevant " $\mathrm{J}$ " qualifier that appears in the Hanford Environmental Information System

(HEIS) database and in analytical data. The data quality assessment (Appendix B) further discusses the "J" qualifiers applied during validation.

$\mathrm{U}=$ Below laboratory detection limits 
Table A-2. Northern Portion Overburden Cleanup Verification Data (Sample Date 7/15/02).

\begin{tabular}{|c|c|c|c|c|c|c|c|c|c|c|c|c|c|c|c|c|c|}
\hline \multirow{2}{*}{$\begin{array}{c}\text { Sampling } \\
\text { Area }\end{array}$} & \multirow{2}{*}{$\begin{array}{c}\text { HEIS } \\
\text { Number }\end{array}$} & \multirow{2}{*}{$\begin{array}{c}\text { Sample } \\
\text { Date }\end{array}$} & \multicolumn{3}{|c|}{ Cs-137 } & \multicolumn{3}{|c|}{ Co-60 } & \multicolumn{3}{|c|}{ Eu-152 } & \multicolumn{3}{|c|}{ Eu-154 } & \multicolumn{3}{|c|}{ Eu-155 } \\
\hline & & & $\mathrm{pCi} / \mathrm{g}$ & $\mathbf{Q}$ & MDA & $\mathrm{pCi} / \mathrm{g}$ & $\mathbf{Q}$ & MDA & $\mathrm{pCi} / \mathrm{g}$ & $\mathbf{Q}$ & MDA & $\mathrm{pCi} / \mathrm{g}$ & $\mathbf{Q}$ & MDA & $\mathrm{pCi} / \mathrm{g}$ & $\mathbf{Q}$ & MDA \\
\hline A1 & $\mathrm{J} 00097$ & $07 / 15 / 2002$ & $4.90 \mathrm{E}-02$ & $U$ & $4.90 \mathrm{E}-02$ & $5.50 \mathrm{E}-02$ & $U$ & $5.50 \mathrm{E}-02$ & $1.00 \mathrm{E}-01$ & $U$ & $1.00 \mathrm{E}-01$ & 1.80E-01 & $U$ & $1.80 \mathrm{E}-01$ & 8.10E-02 & $U$ & $8.10 \mathrm{E}-02$ \\
\hline $\begin{array}{c}\text { Duplicate of } \\
\text { J00097 }\end{array}$ & J000B9 & $07 / 15 / 2002$ & $1.10 \mathrm{E}-01$ & $U$ & 1.10E-01 & $3.10 \mathrm{E}-02$ & $U$ & $3.10 \mathrm{E}-02$ & $9.10 \mathrm{E}-02$ & $U$ & $9.10 \mathrm{E}-02$ & $1.00 \mathrm{E}-01$ & U & $1.00 \mathrm{E}-01$ & $1.40 \mathrm{E}-01$ & $U$ & $1.40 \mathrm{E}-01$ \\
\hline $\begin{array}{l}\text { Split of } \\
\text { J00097 }\end{array}$ & J000C1 & $07 / 15 / 2002$ & 1.4E-02 & $U$ & 2.2E-02 & $6.5 E-03$ & $\cup$ & 2.1E-02 & $-3.7 E-03$ & $U$ & 4.7E-02 & -5.7E-02 & U & 6.3E-02 & 3.1E-02 & $U$ & 4.7E-02 \\
\hline $\mathrm{A} 2$ & $\mathrm{~J} 00098$ & $07 / 15 / 2002$ & $6.60 \mathrm{E}-02$ & $a$ & $3.60 \mathrm{E}-02$ & $3.20 \mathrm{E}-02$ & $U$ & $3.20 \mathrm{E}-02$ & 7.00E-02 & $U$ & $7.00 \mathrm{E}-02$ & $1.10 \mathrm{E}-01$ & $U$ & 1.10E-01 & 6.30E-02 & $U$ & $6.30 \mathrm{E}-02$ \\
\hline $\mathrm{A} 3$ & J00099 & $07 / 15 / 2002$ & $4.80 \mathrm{E}-02$ & $U$ & $4.80 \mathrm{E}-02$ & $5.10 \mathrm{E}-02$ & $U$ & $5.10 \mathrm{E}-02$ & $1.20 \mathrm{E}-01$ & $U$ & $1.20 \mathrm{E}-01$ & $1.80 \mathrm{E}-01$ & $U$ & $1.80 \mathrm{E}-01$ & $1.10 \mathrm{E}-01$ & $U$ & $1.10 \mathrm{E}-01$ \\
\hline A4 & J000B0 & $07 / 15 / 2002$ & $1.07 \mathrm{E}-01$ & & $4.00 \mathrm{E}-02$ & $3.80 \mathrm{E}-02$ & $U$ & $3.80 \mathrm{E}-02$ & $2.10 \mathrm{E}-01$ & $U$ & $2.10 \mathrm{E}-01$ & $1.30 \mathrm{E}-01$ & $\mathrm{U}$ & $1.30 \mathrm{E}-01$ & $1.20 \mathrm{E}-01$ & $U$ & $1.20 \mathrm{E}-01$ \\
\hline B5 & J000B1 & $07 / 15 / 2002$ & $5.00 \mathrm{E}-02$ & $a$ & $2.90 \mathrm{E}-02$ & $3.60 \mathrm{E}-02$ & $U$ & $3.60 \mathrm{E}-02$ & 1.10E-01 & $U$ & $1.10 \mathrm{E}-01$ & $1.20 \mathrm{E}-01$ & $\mathrm{U}$ & $1.20 \mathrm{E}-01$ & $1.10 \mathrm{E}-01$ & $U$ & 1.10E-01 \\
\hline B6 & J000B2 & $07 / 15 / 2002$ & 8.70E-02 & $a$ & 4.30E-02 & $5.30 \mathrm{E}-02$ & $U$ & 5.30E-02 & $2.00 E-01$ & $U$ & $2.00 \mathrm{E}-01$ & $1.70 \mathrm{E}-01$ & $U$ & 1.70E-01 & $7.80 \mathrm{E}-02$ & $U$ & $7.80 \mathrm{E}-02$ \\
\hline B7 & J000B3 & $07 / 15 / 2002$ & 1.11E-01 & & $3.50 \mathrm{E}-02$ & $3.40 \mathrm{E}-02$ & $U$ & $3.40 \mathrm{E}-02$ & 1.73E-01 & & $6.30 \mathrm{E}-02$ & 1.10E-01 & $U$ & 1.10E-01 & $5.90 \mathrm{E}-02$ & $U$ & $5.90 \mathrm{E}-02$ \\
\hline B8 & J000B4 & $07 / 15 / 2002$ & $7.30 \mathrm{E}-02$ & $a$ & $4.20 \mathrm{E}-02$ & $5.00 \mathrm{E}-02$ & $U$ & $5.00 \mathrm{E}-02$ & $2.56 \mathrm{E}-01$ & & $1.00 \mathrm{E}-01$ & $1.60 \mathrm{E}-01$ & $U$ & $1.60 \mathrm{E}-01$ & $1.10 \mathrm{E}-01$ & $U$ & $1.10 \mathrm{E}-01$ \\
\hline $\mathrm{C} 9$ & J000B5 & $07 / 15 / 2002$ & $4.50 \mathrm{E}-02$ & $\mathrm{U}$ & $4.50 \mathrm{E}-02$ & $4.50 \mathrm{E}-02$ & $U$ & $4.50 \mathrm{E}-02$ & $1.20 \mathrm{E}-01$ & $\mathrm{U}$ & $1.20 \mathrm{E}-01$ & $1.60 \mathrm{E}-01$ & $U$ & $1.60 \mathrm{E}-01$ & 1.10E-01 & $U$ & $1.10 \mathrm{E}-01$ \\
\hline $\mathrm{C} 10$ & J000B6 & $07 / 15 / 2002$ & 1.97E-01 & & 4.70E-02 & $8.10 E-02$ & & $4.00 \mathrm{E}-01$ & $9.56 \mathrm{E}-01$ & & $9.40 \mathrm{E}-02$ & $1.50 \mathrm{E}-01$ & $U$ & 1.50E-01 & 1.40E-01 & $U$ & 1.40E-01 \\
\hline $\mathrm{C} 1$ & J000B7 & $07 / 15 / 2002$ & $6.70 \mathrm{E}-02$ & $U$ & $6.70 \mathrm{E}-02$ & $5.30 \mathrm{E}-02$ & $U$ & $5.30 \mathrm{E}-02$ & $1.10 \mathrm{E}-01$ & $U$ & $1.10 \mathrm{E}-01$ & $1.70 \mathrm{E}-01$ & $U$ & 1.70E-01 & $8.00 \mathrm{E}-02$ & $\mathrm{U}$ & $8.00 \mathrm{E}-02$ \\
\hline $\mathrm{C} 2$ & J000B8 & $07 / 15 / 2002$ & $6.80 \mathrm{E}-02$ & $a$ & $3.30 E-02$ & $3.30 E-02$ & $U$ & $3.30 \mathrm{E}-02$ & $1.02 E-01$ & & $6.00 \mathrm{E}-02$ & $1.10 \mathrm{E}-01$ & $U$ & $1.10 \mathrm{E}-01$ & $6.00 \mathrm{E}-02$ & $U$ & $6.00 E-02$ \\
\hline
\end{tabular}

"Because of laboratory reporting conditions, these data were given a nonrelevant "J" qualifier that appears in the Hanford Environmental Information System (HEIS) database and in analytical data. The data quality assessment (Appendix B) further discusses the " $\mathrm{J"} \mathrm{qualifiers} \mathrm{applied} \mathrm{during} \mathrm{validation}$

$\mathrm{U}=$ Below laboratory detection limits 
Table A-3. Southern Portion Shallow Zone Cleanup Verification Data (Sample Date 5/21/01, 6/18/01). (2 pages)

\begin{tabular}{|c|c|c|c|c|c|c|c|c|c|c|c|c|c|c|c|c|c|}
\hline \multirow{2}{*}{$\begin{array}{c}\text { Sample } \\
\text { Area }\end{array}$} & \multirow{2}{*}{$\begin{array}{c}\text { HEIS } \\
\text { Number }\end{array}$} & \multirow{2}{*}{$\begin{array}{c}\text { Sample } \\
\text { Date }\end{array}$} & \multicolumn{3}{|c|}{ Cs-137 } & \multicolumn{3}{|c|}{ Co-60 } & \multicolumn{3}{|c|}{ Eu-152 } & \multicolumn{3}{|c|}{ Eu-154 } & \multicolumn{3}{|c|}{ Eu-155 } \\
\hline & & & $\mathrm{pCi} / \mathrm{g}$ & $Q$ & MDA & $\mathrm{pCi} / \mathrm{g}$ & Q & MDA & $\mathrm{pCi} / \mathrm{g}$ & Q & MDA & $\mathrm{pCi} / \mathrm{g}$ & $\mathbf{Q}$ & MDA & $\mathrm{pCi} / \mathrm{g}$ & $\mathbf{Q}$ & MDA \\
\hline 2 & B121M4 & $5 / 21 / 2001$ & $1.50 \mathrm{E}-01$ & & $7.20 \mathrm{E}-02$ & $1.36 \mathrm{E}-01$ & & $7.40 \mathrm{E}-02$ & $9.57 \mathrm{E}-01$ & & $1.80 \mathrm{E}-01$ & $2.50 \mathrm{E}-01$ & $U$ & $2.50 \mathrm{E}-01$ & $1.80 \mathrm{E}-01$ & $U$ & $1.80 \mathrm{E}-01$ \\
\hline $\begin{array}{c}\text { Duplicate } \\
\text { of B121M4 }\end{array}$ & B121P8 & $5 / 21 / 2001$ & 1.44E-01 & & $5.80 \mathrm{E}-02$ & $5.40 \mathrm{E}-02$ & $u$ & $5.40 \mathrm{E}-02$ & $1.50 \mathrm{E}-01$ & $u$ & $1.50 \mathrm{E}-01$ & $1.50 \mathrm{E}-01$ & $u \mid$ & $1.50 \mathrm{E}-01$ & $1.80 \mathrm{E}-01$ & $u \mid$ & $1.80 \mathrm{E}-01$ \\
\hline 17 & B121N9 & $5 / 21 / 2001$ & $8.70 \mathrm{E}-02$ & a & $4.20 \mathrm{E}-02$ & $3.50 \mathrm{E}-02$ & $u$ & \begin{tabular}{|l|}
$3.50 \mathrm{E}-02$ \\
\end{tabular} & $1.02 \mathrm{E}-01$ & & $7.50 \mathrm{E}-02$ & $1.00 \mathrm{E}-01$ & $u$ & $1.00 \mathrm{E}-01$ & 1.10E-01 & $U$ & $1.10 \mathrm{E}-01$ \\
\hline $\begin{array}{c}\text { Duplicate } \\
\text { of B121N9 }\end{array}$ & B121P7 & $5 / 21 / 2001$ & $4.90 \mathrm{E}-02$ & $u$ & $4.90 \mathrm{E}-02$ & 4.90E-02 & $u$ & 4.90E-02 & $2.00 \mathrm{E}-01$ & $u$ & $2.00 \mathrm{E}-01$ & $1.20 \mathrm{E}-01$ & $U$ & $1.20 \mathrm{E}-01$ & $1.40 \mathrm{E}-01$ & $u$ & $1.40 \mathrm{E}-01$ \\
\hline \multirow{2}{*}{13} & B121N5 & $5 / 21 / 2001$ & $1.10 \mathrm{E}+00$ & & $1.60 \mathrm{E}-01$ & 1.63E+00 & & $1.00 \mathrm{E}-01$ & $1.46 \mathrm{E}+01$ & & $2.20 \mathrm{E}-01$ & $1.73 E+00$ & & $3.40 \mathrm{E}-01$ & $2.40 \mathrm{E}-01$ & $\mathrm{U}$ & 2.40E-01 \\
\hline & B12688 & $6 / 18 / 2001$ & $5.83 \mathrm{E}-01$ & & $3.00 \mathrm{E}-01$ & $7.34 \mathrm{E}-01$ & & $2.30 \mathrm{E}-01$ & $6.84 E+00$ & & $3.70 \mathrm{E}-01$ & $6.50 \mathrm{E}-01$ & & $6.90 \mathrm{E}-01$ & $3.30 \mathrm{E}-01$ & $U$ & $3.30 \mathrm{E}-01$ \\
\hline $\begin{array}{c}\text { Duplicate } \\
\text { of B12688 }\end{array}$ & B12692 & 6/18/2001 & $9.68 \mathrm{E}-01$ & & $1.60 \mathrm{E}-01$ & $6.24 \mathrm{E}-01$ & & 1.50E-01 & $6.46 E+00$ & & 3.60E-01 & $1.01 \mathrm{E}+00$ & & $4.60 \mathrm{E}-01$ & $5.70 \mathrm{E}-01$ & $u$ & $5.70 \mathrm{E}-01$ \\
\hline 19 & B121P1 & $5 / 21 / 2001$ & $7.10 \mathrm{E}-02$ & a & $2.80 \mathrm{E}-02$ & $5.80 \mathrm{E}-02$ & $\mathrm{U}$ & $5.80 \mathrm{E}-02$ & 2.07E-01 & & $1.00 \mathrm{E}-01$ & $1.20 \mathrm{E}-01$ & $u$ & $1.20 \mathrm{E}-01$ & $1.10 \mathrm{E}-01$ & $u$ & $1.10 \mathrm{E}-01$ \\
\hline 20 & $\mathrm{~B} 121 \mathrm{P} 2$ & $5 / 21 / 2001$ & $1.02 \mathrm{E}-01$ & & $2.30 \mathrm{E}-02$ & $1.80 \mathrm{E}-02$ & $U$ & $1.80 \mathrm{E}-02$ & $7.10 \mathrm{E}-02$ & 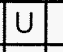 & $7.10 \mathrm{E}-02$ & $5.50 \mathrm{E}-02$ & $u$ & $5.50 \mathrm{E}-02$ & $5.60 \mathrm{E}-02$ & $U$ & $5.60 \mathrm{E}-02$ \\
\hline 21 & $\mathrm{~B} 121 \mathrm{P} 3$ & $5 / 21 / 2001$ & $1.16 \mathrm{E}-01$ & & $3.30 \mathrm{E}-02$ & $2.50 \mathrm{E}-02$ & $\mathrm{U}$ & $2.50 \mathrm{E}-02$ & $1.10 \mathrm{E}-01$ & $U$ & $1.10 \mathrm{E}-01$ & $7.80 \mathrm{E}-02$ & $U$ & $7.80 \mathrm{E}-02$ & $8.80 \mathrm{E}-02$ & $u$ & $8.80 \mathrm{E}-02$ \\
\hline 22 & B121P4 & $5 / 21 / 2001$ & $1.41 E+00$ & & $1.70 \mathrm{E}-01$ & $1.06 \mathrm{E}+00$ & & $9.20 \mathrm{E}-02$ & $1.53 \mathrm{E}+01$ & & $2.40 \mathrm{E}-01$ & $1.90 \mathrm{E}+00$ & & $3.30 \mathrm{E}-01$ & $4.60 \mathrm{E}-01$ & $U$ & $4.60 \mathrm{E}-01$ \\
\hline 23 & B121P5 & $5 / 21 / 2001$ & $2.60 \mathrm{E}-01$ & & $7.70 \mathrm{E}-02$ & $9.50 \mathrm{E}-02$ & & $5.50 \mathrm{E}-02$ & $2.62 E+00$ & & $1.40 \mathrm{E}-01$ & $3.17 \mathrm{E}-01$ & & $2.00 \mathrm{E}-01$ & $1.80 \mathrm{E}-01$ & $U$ & $1.80 \mathrm{E}-01$ \\
\hline 15 & B121N7 & $5 / 21 / 2001$ & $2.37 \mathrm{E}-01$ & & $4.40 \mathrm{E}-02$ & $6.40 \mathrm{E}-02$ & & $4.10 \mathrm{E}-02$ & $1.30 \mathrm{E}+00$ & & $7.90 \mathrm{E}-02$ & $1.20 \mathrm{E}-01$ & $u$ & $1.20 \mathrm{E}-01$ & $1.10 \mathrm{E}-01$ & $u$ & $1.10 \mathrm{E}-01$ \\
\hline 14 & B121N6 & $5 / 21 / 2001$ & $9.50 \mathrm{E}-02$ & a & $6.00 \mathrm{E}-02$ & $4.60 \mathrm{E}-02$ & U & $4.60 \mathrm{E}-02$ & $1.30 \mathrm{E}-01$ & $u$ & $1.30 \mathrm{E}-01$ & $1.60 \mathrm{E}-01$ & $u$ & $1.60 \mathrm{E}-01$ & $1.50 \mathrm{E}-01$ & $U$ & $1.50 \mathrm{E}-01$ \\
\hline 7 & B121M9 & $5 / 21 / 2001$ & $7.70 \mathrm{E}-02$ & $\mathrm{U}$ & $7.70 \mathrm{E}-02$ & $8.30 \mathrm{E}-02$ & $U$ & $8.30 \mathrm{E}-02$ & $2.10 \mathrm{E}-01$ & $U$ & $2.10 \mathrm{E}-01$ & $2.20 \mathrm{E}-01$ & $\mathrm{u}$ & $2.20 \mathrm{E}-01$ & $1.90 \mathrm{E}-01$ & 0 & $1.90 \mathrm{E}-01$ \\
\hline 12 & B121N4 & $5 / 21 / 2001$ & $8.30 \mathrm{E}-02$ & $U$ & $8.30 \mathrm{E}-02$ & $7.50 \mathrm{E}-02$ & $u$ & $7.50 \mathrm{E}-02$ & $1.80 \mathrm{E}-01$ & $U$ & $1.80 \mathrm{E}-01$ & $2.10 \mathrm{E}-01$ & $u$ & $2.10 \mathrm{E}-01$ & $2.40 \mathrm{E}-01$ & $u$ & $2.40 \mathrm{E}-01$ \\
\hline 11 & B121N3 & $5 / 21 / 2001$ & $1.01 \mathrm{E}-01$ & & $4.50 \mathrm{E}-02$ & $5.20 \mathrm{E}-02$ & $\mathrm{U}$ & $5.20 \mathrm{E}-02$ & $1.90 \mathrm{E}-01$ & $U$ & $1.90 \mathrm{E}-01$ & $1.40 \mathrm{E}-01$ & $\mathrm{U}$ & $1.40 \mathrm{E}-01$ & $1.90 \mathrm{E}-01$ & $u$ & $1.90 \mathrm{E}-01$ \\
\hline 6 & $\mathrm{~B} 121 \mathrm{M} 8$ & $5 / 21 / 2001$ & $6.20 \mathrm{E}-02$ & a & $2.90 \mathrm{E}-02$ & $3.50 \mathrm{E}-02$ & U & $3.50 \mathrm{E}-02$ & $9.40 \mathrm{E}-02$ & $\mathrm{U}$ & $9.40 \mathrm{E}-02$ & $1.00 \mathrm{E}-01$ & $u$ & $1.00 \mathrm{E}-01$ & $1.10 \mathrm{E}-01$ & $U$ & $1.10 \mathrm{E}-01$ \\
\hline \multirow{2}{*}{5} & B121M7 & $5 / 21 / 2001$ & $7.84 \mathrm{E}-01$ & & $9.70 \mathrm{E}-02$ & $4.94 \mathrm{E}-01$ & & $7.40 \mathrm{E}-02$ & $7.22 \mathrm{E}+00$ & & $1.70 \mathrm{E}-01$ & $7.91 \mathrm{E}-01$ & & $2.00 \mathrm{E}-01$ & $2.10 \mathrm{E}-01$ & U & $2.10 \mathrm{E}-01$ \\
\hline & B12689 & $6 / 18 / 2001$ & $2.50 \mathrm{E}-01$ & & 1.10E-01 & $2.33 \mathrm{E}-01$ & & $9.30 \mathrm{E}-02$ & $2.71 \mathrm{E}+00$ & & $2.00 \mathrm{E}-01$ & $3.93 \mathrm{E}-01$ & & 3.40E-01 & $3.30 \mathrm{E}-01$ & $u$ & $3.30 \mathrm{E}-01$ \\
\hline 10 & B121N2 & $5 / 21 / 2001$ & 1.42E-01 & & $7.40 \mathrm{E}-02$ & $9.70 \mathrm{E}-02$ & & $5.90 \mathrm{E}-02$ & $8.27 \mathrm{E}-01$ & & $1.20 \mathrm{E}-01$ & $1.90 \mathrm{E}-01$ & $\mathrm{U}$ & $1.90 \mathrm{E}-01$ & $2.00 \mathrm{E}-01$ & $\mathrm{U}$ & $2.00 \mathrm{E}-01$ \\
\hline \multirow[t]{2}{*}{4} & $\mathrm{~B} 121 \mathrm{M} 6$ & $5 / 21 / 2001$ & $4.04 \mathrm{E}-01$ & & $4.80 \mathrm{E}-02$ & $2.92 \mathrm{E}-01$ & & $3.30 \mathrm{E}-02$ & $3.88 \mathrm{E}+00$ & & $8.20 \mathrm{E}-02$ & $6.01 \mathrm{E}-01$ & & $1.20 \mathrm{E}-01$ & 1.10E-01 & $u$ & $1.10 \mathrm{E}-01$ \\
\hline & $\mathrm{B} 12690$ & $6 / 18 / 2001$ & $3.19 \mathrm{E}-01$ & & $1.70 \mathrm{E}-01$ & $2.40 \mathrm{E}-01$ & $u$ & $2.40 \mathrm{E}-01$ & $1.92 \mathrm{E}+00$ & & $3.60 \mathrm{E}-01$ & $5.80 \mathrm{E}-01$ & $U$ & $5.80 \mathrm{E}-01$ & $2.60 \mathrm{E}-01$ & U & $2.60 \mathrm{E}-01$ \\
\hline 3 & B121M5 & $5 / 21 / 2001$ & $1.92 \mathrm{E}-01$ & & $4.80 \mathrm{E}-02$ & $4.90 \mathrm{E}-02$ & $a$ & $3.90 \mathrm{E}-02$ & $8.94 \mathrm{E}-01$ & & $1.10 \mathrm{E}-01$ & $1.40 \mathrm{E}-01$ & U & $1.40 \mathrm{E}-01$ & $1.30 \mathrm{E}-01$ & $u$ & $1.30 \mathrm{E}-01$ \\
\hline 24 & $\mathrm{~B} 121 \mathrm{P} 6$ & $5 / 21 / 2001$ & $2.20 \mathrm{E}-01$ & & $4.80 \mathrm{E}-02$ & $4.80 \mathrm{E}-02$ & U & $4.80 \mathrm{E}-02$ & $1.10 \mathrm{E}-01$ & $u$ & $1.10 \mathrm{E}-01$ & $1.50 \mathrm{E}-01$ & $U$ & $1.50 \mathrm{E}-01$ & $1.70 \mathrm{E}-01$ & 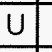 & $1.70 \mathrm{E}-01$ \\
\hline 9 & $\mathrm{~B} 121 \mathrm{~N} 1$ & $5 / 21 / 2001$ & $3.76 \mathrm{E}-01$ & & $6.80 \mathrm{E}-02$ & $2.14 \mathrm{E}-01$ & & $4.00 \mathrm{E}-02$ & $3.15 E+00$ & & $1.10 \mathrm{E}-01$ & $3.30 \mathrm{E}-01$ & & $1.80 \mathrm{E}-01$ & $1.50 \mathrm{E}-01$ & $u$ & $1.50 \mathrm{E}-01$ \\
\hline 1 & $\mathrm{~B} 121 \mathrm{M} 3$ & $5 / 21 / 2001$ & $3.32 \mathrm{E}-01$ & & $8.40 \mathrm{E}-02$ & $1.41 \mathrm{E}-01$ & & $6.70 \mathrm{E}-02$ & $1.79 \mathrm{E}+00$ & & $1.40 \mathrm{E}-01$ & $2.80 \mathrm{E}-01$ & $U$ & $2.80 \mathrm{E}-01$ & $1.10 \mathrm{E}-01$ & $u$ & $1.10 \mathrm{E}-01$ \\
\hline \multirow{2}{*}{8} & B121NO & $5 / 21 / 2001$ & $8.52 \mathrm{E}-01$ & & $1.00 \mathrm{E}-01$ & 4.43E-01 & & $4.80 \mathrm{E}-02$ & $9.08 E+00$ & & $1.70 \mathrm{E}-01$ & $9.36 \mathrm{E}-01$ & & $2.00 \mathrm{E}-01$ & $2.40 \mathrm{E}-01$ & $u$ & $2.40 \mathrm{E}-01$ \\
\hline & B12691 & 6/18/2001 & $6.34 \mathrm{E}-01$ & & 2.50E-01 & $4.88 \mathrm{E}-01$ & & 1.10E-01 & $9.12 \mathrm{E}+00$ & & $4.70 \mathrm{E}-01$ & $1.24 \mathrm{E}+00$ & & $4.10 \mathrm{E}-01$ & $8.00 \mathrm{E}-01$ & $u$ & $8.00 \mathrm{E}-01$ \\
\hline 16 & B121N8 & $5 / 21 / 2001$ & $1.71 \mathrm{E}-01$ & & $6.40 \mathrm{E}-02$ & $5.50 \mathrm{E}-02$ & 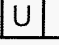 & $5.50 \mathrm{E}-02$ & $1.40 \mathrm{E}-01$ & $U$ & $1.40 \mathrm{E}-01$ & 1.70E-01 & $U$ & $1.70 \mathrm{E}-01$ & $1.30 \mathrm{E}-01$ & $u$ & $1.30 \mathrm{E}-01$ \\
\hline
\end{tabular}


Table A-3. Southern Portion Shallow Zone Cleanup Verification Data (Sample Date 5/21/01, 6/18/01). (2 pages)

\begin{tabular}{|c|c|c|c|c|c|c|c|c|c|c|c|c|c|c|c|c|c|}
\hline \multirow{2}{*}{$\begin{array}{c}\text { Sample } \\
\text { Area }\end{array}$} & \multirow{2}{*}{$\begin{array}{c}\text { HEIS } \\
\text { Number }\end{array}$} & \multirow{2}{*}{$\begin{array}{l}\text { Sample } \\
\text { Date }\end{array}$} & \multicolumn{3}{|c|}{ Cs-137 } & \multicolumn{3}{|c|}{ Co-60 } & \multicolumn{3}{|c|}{ Eu-152 } & \multicolumn{3}{|c|}{ Eu-154 } & \multicolumn{3}{|c|}{ Eu-155 } \\
\hline & & & $\mathrm{pCi} / \mathrm{g}$ & $\mathbf{Q}$ & MDA & $\mathrm{pCi} / \mathrm{g}$ & $\mathbf{Q}$ & MDA & $\mathrm{pCi} / \mathrm{g}$ & $\mathbf{Q}$ & MDA & $\mathrm{pCi} / \mathrm{g}$ & Q & MDA & $\mathrm{pCi} / \mathrm{g}$ & $\mathbf{Q}$ & MDA \\
\hline 18 & B121P0 & $5 / 21 / 2001$ & $8.80 \mathrm{E}-02$ & $a$ & $5.70 \mathrm{E}-02$ & $4.80 \mathrm{E}-02$ & $U$ & $4.80 \mathrm{E}-02$ & $2.50 E-01$ & $\mathrm{U}$ & $2.50 \mathrm{E}-01$ & $1.50 E-01$ & $u$ & $1.50 \mathrm{E}-01$ & $1.70 \mathrm{E}-01$ & $u$ & $1.70 \mathrm{E}-01$ \\
\hline
\end{tabular}

"Because of laboratory reporting conditions, these data were given a nonrelevant " $J$ " qualifier that appears in the Hanford Environmental Information System (HEIS) database and in analytical data. The data quality assessment (Appendix B) further discusses the "J" qualifiers applied during validation.

$U=$ Below laboratory detection limits 
CVP-2002-00004

Rev. 1

A-6 
APPENDIX B

DATA QUALITY ASSESSMENT

$B-i$ 
CVP-2002-00004

Rev. 1

B-ii 


\section{B1.0 DATA QUALITY ASSESSMENT FOR THE 126-F-1 SITE}

\section{B1.1 OVERVIEW}

This data quality assessment (DQA) was performed to compare the verification sampling approach and resulting analytical data with the sampling and data quality requirements specified by the project objectives and performance specifications. The DQA involves the scientific and statistical evaluation of the data to determine if they are of the right type, quality, and quantity to support their intended use (i.e., closeout decisions [EPA 1996]). The DQA completes the data life cycle (i.e., planning, implementation, and assessment) that was initiated by the data quality objectives process.

This DQA was performed in accordance with BHI-EE-01, Environmental Investigations Procedures. Specific data quality objectives for the site are found in the 100 Area Remedial Action Sampling and Analysis Plan (SAP) (DOE-RL 2001). The DQA is based on the guidelines presented in Guidance for Data Quality Assessment (EPA 2000). Statistical tests used in this DQA were performed as specified in the SAP (DOE-RL 2001) and the Remedial Design Report/Remedial Action Work Plan for the 100 Area (RDR/RAWP) (DOE-RL 2005).

Prior to performing statistical tests, all of the analytical data are evaluated and at least a portion are validated for compliance with quality assurance project plan requirements (DOE-RL 2001). Data evaluation is performed to determine if the laboratory carried out all steps required by the SAP and the laboratory contract governing the conduct of analysis and reporting of the data. This evaluation also examines the available laboratory data to determine if an analyte is present or absent in a sample and the degree of overall uncertainty associated with that determination. Data validation is done in accordance with validation procedures (BHI 2000a, 2000b) as part of the data evaluation. After data evaluation and validation, the appropriate statistical test is performed on the adjusted raw analytical data (see calculation briefs in Appendix C), to determine statistical values for each contaminant. The number of samples collected for cleanup verification is then evaluated to confirm assumptions concerning contaminant variability.

The DQA for the 126-F-1 site determined that the data are of the right type, quality, and quantity to support site cleanup verification decisions within specified error tolerances. All analytical data were found to be acceptable for decision-making purposes. The evaluation verified that the sample design was sufficient for the purpose of clean site verification. Additional quality requirements of the quality assurance project plan included data acquisition requirements. The cleanup verification sample analytical data are stored in the Hanford Environmental Information System and are summarized in Appendix A.

The following subsections describe the DQA results for the 126-F-1 site, including formal data validation, supplementary data evaluation, and field quality assurance 
$(\mathrm{QA})$ /quality control $(\mathrm{QC})$ program results. The statistical evaluation of the data is provided in the calculation brief excerpts included in Appendix C.

\section{B1.2 DATA VALIDATION}

\section{B1.2.1 Laboratory QA/QC Analysis}

All verification samples are subject to laboratory-specific $Q A$ requirements, including instrument procurement, maintenance, calibration, and operation. Additional laboratory requirements for internal QC checks are performed as appropriate for the analytical method at a rate of 1 per sample delivery group (SDG), or 1 in 20, whichever is more frequent. Laboratory internal $\mathrm{QC}$ checks include the following:

- Laboratory Contamination. Each analytical batch contains a laboratory (method) blank (material of similar composition as the samples with $\mathrm{known} / \mathrm{minimal}$ contamination of the analytes of interest) carried through the complete analytical process. The method blank is used to evaluate false-positive results in samples due to contamination during handling at the laboratory.

- Analytical Accuracy. For most analyses, a known quantity of representative analytes of interest (matrix spike [MS]) is added to a separate aliquot of a sample from the analytical batch. The recovery percentage of the added MS is used to evaluate analytical accuracy. For analyses not amenable to MS techniques (e.g., gamma energy analysis) or where analytical recovery is corrected via internal standards (e.g., alpha spectral analyses), accuracy is evaluated from recovery of the QC reference sample (e.g., laboratory control spike [LCS] or blank spike sample).

- Analytical Precision. Separate aliquots removed from the same sample container (replicate samples) are analyzed for each analytical batch. The replicate sample results (evaluated as relative percent differences [RPDs]) are used to assess analytical precision.

- $\mathrm{QC}$ reference samples. A QC reference sample is prepared from an independent standard at a concentration other than that used for calibration, but within the calibration range. Reference samples provide an independent check on analytical technique and methodology.

Verification sample laboratories are also subject to periodic and random assessments of the laboratory performance, systems, and overall program. These assessments are performed by the Washington Closure Hanford Quality Assurance group to ensure that the laboratories are performing to meet laboratory contract requirements. 


\section{B1.2.2 Data Validation Results}

After sampling was completed, all of the fixed-base laboratory data from one SDG, $\mathrm{H} 1868$, were validated to Level C per BHI-EE-01, Procedure 2.5, "Data Package Validation Process." Level C validation procedures are specified in Data Validation Procedure for Radiochemical Analysis (BHI 2000a) and Data Validation Procedure for Chemical Analysis (BHI 2000b). Validation was performed by Tech Law, Inc. (Tech Law 2002).

Under the Level C validation procedure, the following items were reviewed, as appropriate, for each analytical method:

- Sample holding times

- Method blanks

- MS recovery

- Surrogate recovery

- MS/matrix spike duplicate (MSD) results

- Sample replicates

- Associated batch laboratory control sample results

- Data package completeness

- Achievement of target (or contractual) detection limits (TDLs).

Data flagged as estimated (i.e., "J") indicate that the associated concentration is an estimate but that the data may be used for decision-making purposes. Data flagged as below detection limits (i.e., "U") indicate that the contaminant was analyzed for but not detected and the concentration is below the minimum detectable activity (MDA) for radionuclides or the practical quantitation limit (PQL) (i.e., reporting limit) for nonradionuclides. For nonradionuclides, nondetects are reported as the PQL. For radionuclides, nondetects report the actual value obtained from analysis (positive or negative but less than the MDA) except for limited analyses where no value can be calculated. In these cases, the MDA is reported. This situation is applicable for sample results that are below detection limits. All other validated results are considered to be accurate within the standard errors associated with the methods.

The adequacy of laboratory QA/QC was evaluated for precision, accuracy, completeness, and target detection limits pursuant to the SAP (DOE-RL 2001). The organization performing the data validation reported that, of the data given formal validation, the laboratory met the standards for performance for precision $( \pm 30 \%)$, accuracy $( \pm 30 \%)$, and completeness $(>90 \%)$. Comparison of the TDL with the respective MDA or PQL is discussed in Section B1.3.

A summary of deficiencies noted during validation of SDG H1868 follows:

Radionuclides. The DQA noted no major deficiencies. Minor deficiencies noted during validation include the following: 
- Of the 47 missed TDL nondetects, only 5 exceeded the TDL by more than a factor of 2. All missed TDL nondetects in shallow zone samples were less than one-tenth of their associated RAGs. Additional discussion on analyses with missed MDAs is provided in Section B1.3.

\section{B1.3 DATA EVALUATION}

The formal data validation described in the previous section included evaluation of only one SDG; however, a DQA is required for all SDGs. Therefore, a supplementary data evaluation was performed on the remaining SDGs. The following paragraphs include the results of the data evaluation of all SDGs.

To ensure adequate data quality, DQA investigators reviewed the study objectives in the SAP (DOE-RL 2001) to determine the context for assessing the data. The context for assessing the data includes evaluating the sample data using the statistical methodology of the SAP (included in the calculation brief excerpts in Appendix C) and a comparison of analytical results to the precision, accuracy, representativeness, comparability, and completeness (PARCC) parameters as specified in the SAP (DOE$R L$ 2001). This section summarizes the results of the PARCC parameter comparison and presents an evaluation of the affected data.

TDL Comparison: Reported analytical detection levels for non-detected analytes were compared to the TDLs specified in the SAP (DOE-RL 2001). When detected results are obtained, evaluation of detection limits is not performed. The data validation and supplemental data evaluation noted any analyses in which the detection limit (MDA or $P Q L)$ was above the SAP TDLs for nondetected analytes.

The reported MDA was above the TDL for the following COCs:

- Cesium-137: 2 of 6 nondetect results

- Cobalt-60: 34 of 61 nondetect results

- Europium-152: 31 of 32 nondetect results

- Europium-154: 63 of 76 nondetect results

- Europium-155: 56 of 92 nondetect results.

Of the 186 missed TDL nondetects, only 8 exceeded the TDL by more than a factor of 3. All missed TDL nondetects in shallow zone samples were less than one-tenth of their associated RAGs with three exceptions (europium-152 in sample B12690 with a result of $0.58 \mathrm{pCi} / \mathrm{g}[\mathrm{U}]$, europium-152 in sample J000D4 with a result of $0.43 \mathrm{pCi} / \mathrm{g}$ [U], and europium-152 in sample J000D5 with a result of $0.40 \mathrm{pCi} / \mathrm{g}$ [U]; the direct exposure RAG is $3.0 \mathrm{pCi} / \mathrm{g})$. Because the reported values are sufficiently less than the remedial action goals, the associated data are of sufficient quality for decision-making purposes (DOE-RL 2005).

All other nondetected analyses had detection limits below the TDL. 
All other parameters meet acceptance criteria.

\section{B1.4 FIELD QA/QC}

Field QAVQC measures were used to assess potential sources of error and crosscontamination of soil samples that could bias results. Field QA/QC samples included two equipment blanks:

- Sample B121P9, associated with shallow zone main sample B121M5

- Sample J000C0, associated with overburden main sample J00097.

Field QA/QC samples also included five field duplicate samples:

- Sample B121P8, duplicate of southern shallow zone main sample B121M4

- Sample B121P7, duplicate of southern shallow zone main sample B121N9

- Sample B12692, duplicate of southern shallow zone main sample B12688

- Sample J000F0, duplicate of northern shallow zone main sample J000C0

- Sample J000H9, duplicate of northern shallow zone main sample J000F1

- Sample J000B9, duplicate of overburden main sample J00097.

Field QA/QC samples included three field split samples:

- Sample J000J0, split of northern shallow zone main sample J000C0

- Sample J000J1, split of northern shallow zone main sample J000F1

- Sample J000C1, split of overburden main sample J00097.

All main and QA/QC sample results are presented in Appendix A.

\section{B1.4.1 Equipment Blank Samples}

Two equipment blank samples (B121P9, associated with southern shallow zone main sample B121M5; J000C0, associated with overburden main sample J00097) were collected as part of the QA/QC measures. The blank sample results for this site were less than detection for all COCs.

\section{B1.4.2 Field Duplicate Samples}

Duplicate samples were collected to provide a relative measure of the degree of local heterogeneity in the sampling medium, unlike laboratory duplicates that are used to evaluate precision in the analytical process. The field duplicates are evaluated by computing the RPD of the duplicate samples for each COC. Only analytes with values above five times the detection limits for both the main and duplicate samples are compared. 
Based on these criteria, calculation of the RPD was required for results for two cesium-137 pairs, two cobalt-60 pairs, three europium-152 pairs, and one europium-154 pair. All calculated RPDs were within the $+/-30 \%$ range.

\section{B1.4.3 Field Split Samples}

Split samples were collected to provide a relative measure of the degree of variability in the sampling, sample handling, and analytical techniques used by commercial laboratories. The field main and split samples are evaluated by computing the RPD of the split samples for each $\mathrm{COC}$ to determine the usability of the verification data. The U.S. Environmental Protection Agency Contract Laboratory Program duplicate sample comparison methodology, USEPA Contract Laboratory Program National Functional Guidelines for Inorganic Data Review (EPA 1994), is used as an initial test of the data from the splits. Only analytes that had values above five times the TDLs or contract required detection limit for both the main and split sample were compared.

Based on these criteria, calculation of the RPD was required in one split/main pair for europium -152 , the result of which was within the $+/-30 \%$ range.

\section{B1.5 SUITABILITY OF DATA}

The conclusion of the DQA is that the data are of the right type, quality, and quantity to support the intended use. Detection limits, precision, accuracy, and SDG completeness were analyzed to determine if any analytical results should be rejected as a result of QA/QC deficiencies. All COC analytical data were found to be acceptable for decisionmaking purposes, and the raw data are acceptable for calculating the required statistical values.

\section{B2.0 REFERENCES}

BHI, 2000a, Data Validation Procedure for Radiochemical Analysis, BHI-01433, Rev. 0, Bechtel Hanford, Inc., Richland, Washington.

BHI, 2000b, Data Validation Procedure for Chemical Analysis, BHI-01435, Rev. 0, Bechtel Hanford, Inc., Richland, Washington.

BHI-EE-01, Environmental Investigations Procedures, Bechtel Hanford, Inc., Richland, Washington.

DOE-RL, 2001, 100 Area Remedial Action Sampling and Analysis Plan, DOE/RL-96-22, Rev. 3, U.S. Department of Energy, Richland Operations Office, Richland, Washington. 
DOE-RL, 2005, Remedial Design Report/Remedial Action Work Plan for the 100 Area, DOE/RL-96-17, Rev. 5, U.S. Department of Energy, Richland Operations Office, Richland, Washington.

EPA, 1994, USEPA Contract Laboratory Program National Functional Guidelines for Inorganic Data Review, EPA 540/R-94/013, U.S. Environmental Protection Agency, Washington, D.C.

EPA, 1996, Guidance for Data Quality Assessment, EPA QA/G-9, U.S. Environmental Protection Agency, Office of Research and Development, Washington, D.C.

EPA, 2000, Guidance for Data Quality Assessment, EPA QA/G-9, QA00 Update, U.S. Environmental Protection Agency, Office of Research and Development, Washington, D.C.

Tech Law, 2002, Radionuclides - Data Package No. H1868-TR (SDG No. H1868), Tech Law, Inc., Richland, Washington. 
CVP-2002-00004

Rev. 1

B-8 


\section{APPENDIX C}

RESRAD INPUT PARAMETERS

AND CALCULATION BRIEF EXCERPTS 
CVP-2002-00004

Rev. 1

\section{DISCLAIMER FOR CALCULATIONS}

The calculations that are provided in the following appendix have been generated to document compliance with established cleanup levels. These calculations should be used in conjunction with other relevant documents in the administrative record. 


\section{CALCULATION BRIEFS}

The following calculation briefs have been prepared in accordance with ENG-1, Engineering Services, ENG-1-4.5, "Project Calculations," Washington Closure Hanford, Richland, Washington.

126-F-1 Ash Pit Sample Variance Calculation, 0100F-CA-V0119, Rev. 0, Bechtel Hanford, Inc., Richland, Washington.

126-F-1 Overburden and Shallow Zone Sample Design, 0100F-CA-V0061 (Overburden), Rev. 0, 0100F-CA-V0118 (Shallow), Rev. 0, Bechtel Hanford, Inc., Richland, Washington.

126-F-1 Northern Portion Cleanup Verification 95\% UCL Calculations, 0100F-CA-V0144, Rev. 0, Bechtel Hanford, Inc., Richland, Washington.

126-F-1 Southern Portion Cleanup Verification 95\% UCL Calculations, 0100F-CA-V0100, Rev. 0, Bechtel Hanford, Inc., Richland, Washington.

126-F-1 (Northern Portion) RESRAD Calculation, 0100F-CA-V0146, Rev. 0, Bechtel Hanford, Inc., Richland, Washington.

126-F-1 (Southern Portion) RESRAD Calculation, 0100F-CA-V0102, Rev. 1, Bechtel Hanford, Inc., Richland, Washington.

126-F-1 (Northern Portion) Comparison to Drinking Water Standards, 0100F-CA-V0145, Rev. 0 , Bechtel Hanford, Inc., Richland, Washington.

126-F-1 (Southern Portion) Comparison to Drinking Water Standards, 0100F-CA-V0101, Rev. 1, Bechtel Hanford, Inc., Richland, Washington.

NOTE: The calculation briefs referenced in this appendix are kept in the active Washington Closure Hanford project files and are available upon request. When the project is completed, the files will be stored in a U.S. Department of Energy, Richland Operations Office repository. Only excerpts of the calculation briefs are included in this appendix. 
CVP-2002-00004

Rev. 1

C-iv 


\section{CALCULATION COVER SHEET}

Project Title:

Area

Discipline

Subject

Computer Program
100-F Group 4 Remedial Action 100-F Remedial Action

Environmental

126-F-1Ash Pit Sample Variance Calculation

Excel
Job No.

22192
Program No. Excel 97

\section{Committed Calculation}

$\mathrm{X}$

Preliminary

Superseded

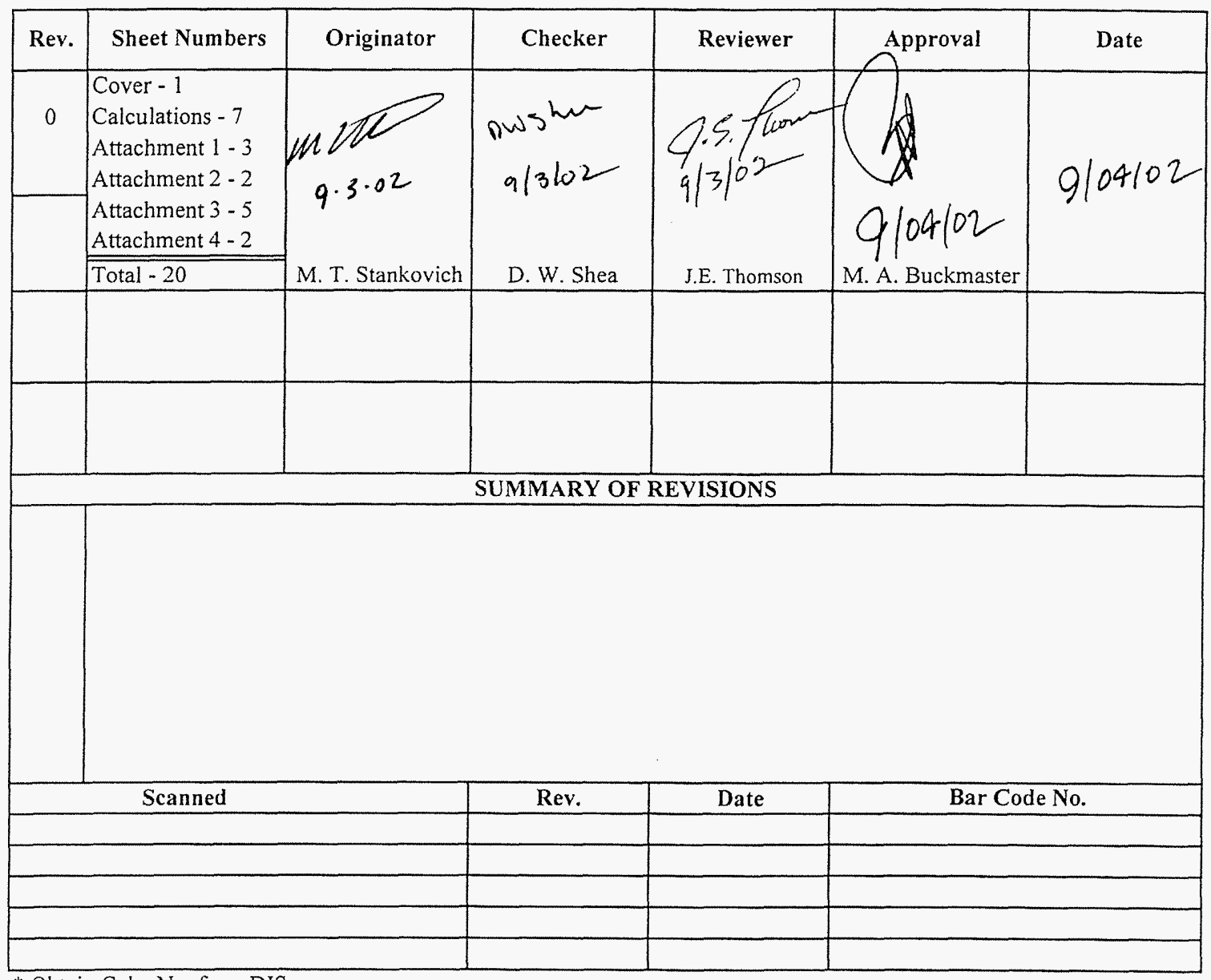




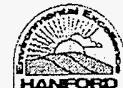

Hanforom Bechtel Hanford Inc

\section{CALCULATION SHEET}

Originator M.T. Stankovich WO Bate

7/10/2002 Calc. No, 0100F-CA-V0119

Rev. No. 0

Project $100 \mathrm{~F}$ Group 4 Remedial Action Job No.

22192 Checked

Dio0F-CA-V01

Date

$7 / 3 / \sqrt{2}$ Subject $\overline{126-F-1 A s h ~ P i t ~ S a m p l e ~ V a r i a n c e ~ C a l c u l a t i o n ~}$ Sheet No.

, Conclusion:

2 The required number of samples calculated ( 2 samples) for each decision sub-unit is less than the default 3 number (4 samples) specified in the "100 Area Remedial Action Sampling and Analysis Plan" DOE/RL-96-22,

4 Rev 3. Therefore, the default number of samples will be collected from each shallow zone decision sub-unit.

Problem:

Calculate the number of verification samples required for 126-F-1 Ash Pit Shallow Zone and Overburden Zone

Decision Unit verification sampling as required in "100 Area Remedial Action Sampling and Analysis Plan"

(DOE/RL-96-22, Rev 3) and "Instruction Guide for the Remediation of 100 Areas Waste Sites" (0100X-IGG0001, Rev 3).

Given:

1) Sample locations for the 126-F-1 Ash Pit Shallow, and Overburden are identified on the 126-F-1 Ash Pit 4 Shallow Zone and Stockpile Sample Plan, Calculation numbers 0100F-CA-V0118 and 0100F-CA-V0061, s respectively

16 2) Lookup values from DOE/RL-96-22, Rev 3.

17 3) Sample Design requirements from DOE/RL-96-22, Rev 3 and 0100X-IG-G0001, Rev 3

Solution:

20. Calculation methodology is described in Appendix A of DOE/RL-96-22, Rev 3. Data from attached worksheets are used to calculate the required number of verification samples. Variance calculation is based on the same 22 three isotopes used to develop the statistical approach in DOE/RL-96-22, Rev 3. The statistical design is based 23 On the premise that these isotopes are the predominant components of the contamination and are representative 24 of the contamination distribution.

26 Sheet No. Contents Topic

\begin{tabular}{l|lll|}
27 & 1 & Calc. Summary & Summary of Calc Brief \\
28 & $2-5$ & Shallow Zone & Required Number of Samples Calculation \\
29 & $6-7$ & Overburden Zone & Required Number of Samples Calculation \\
30 & Att. 1 & Shallow Zone Formulas & Formulas \\
31 & Att. 2 & Overburden Zone Formulas & Formulas \\
32 & Att. 3 & Shallow Zone Data & Raw Data from Sample Management \\
33 & Att. 4 & Overburden Zone Data & Raw Data from Sample Management \\
34 & & \\
35 & & \\
36 & & \\
37 & & \\
38
\end{tabular}

Calc. Summary 


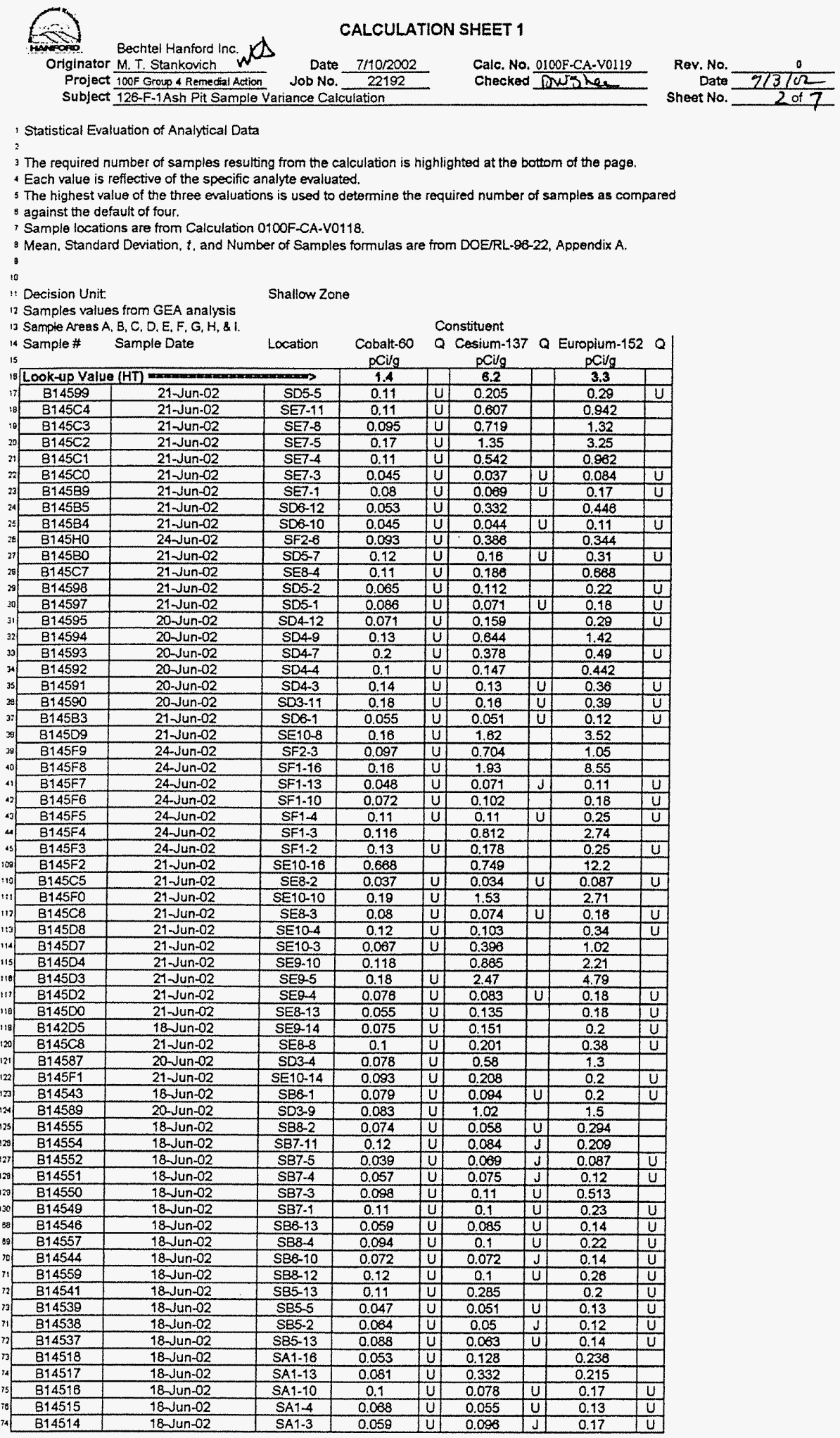

Shallow Zone 


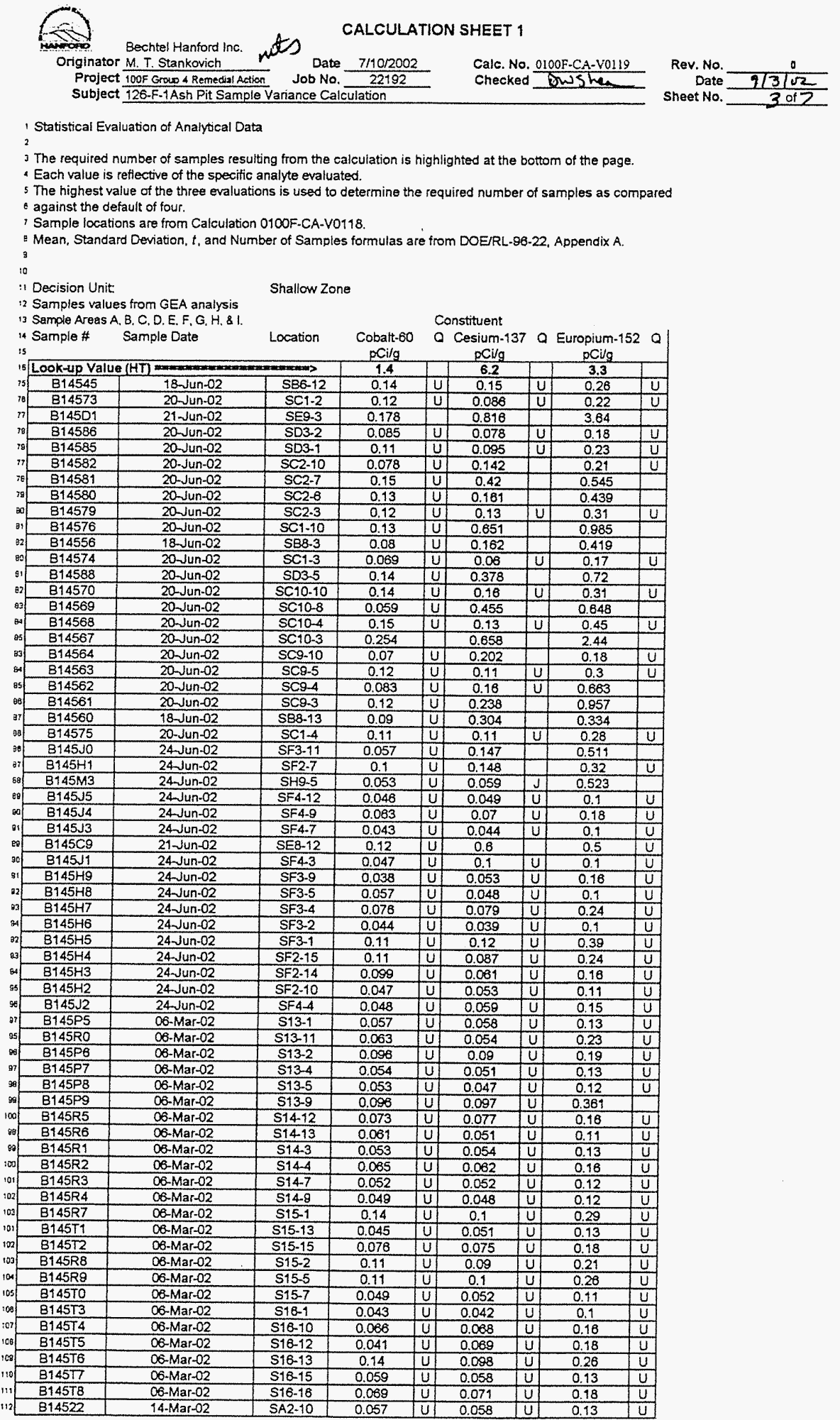




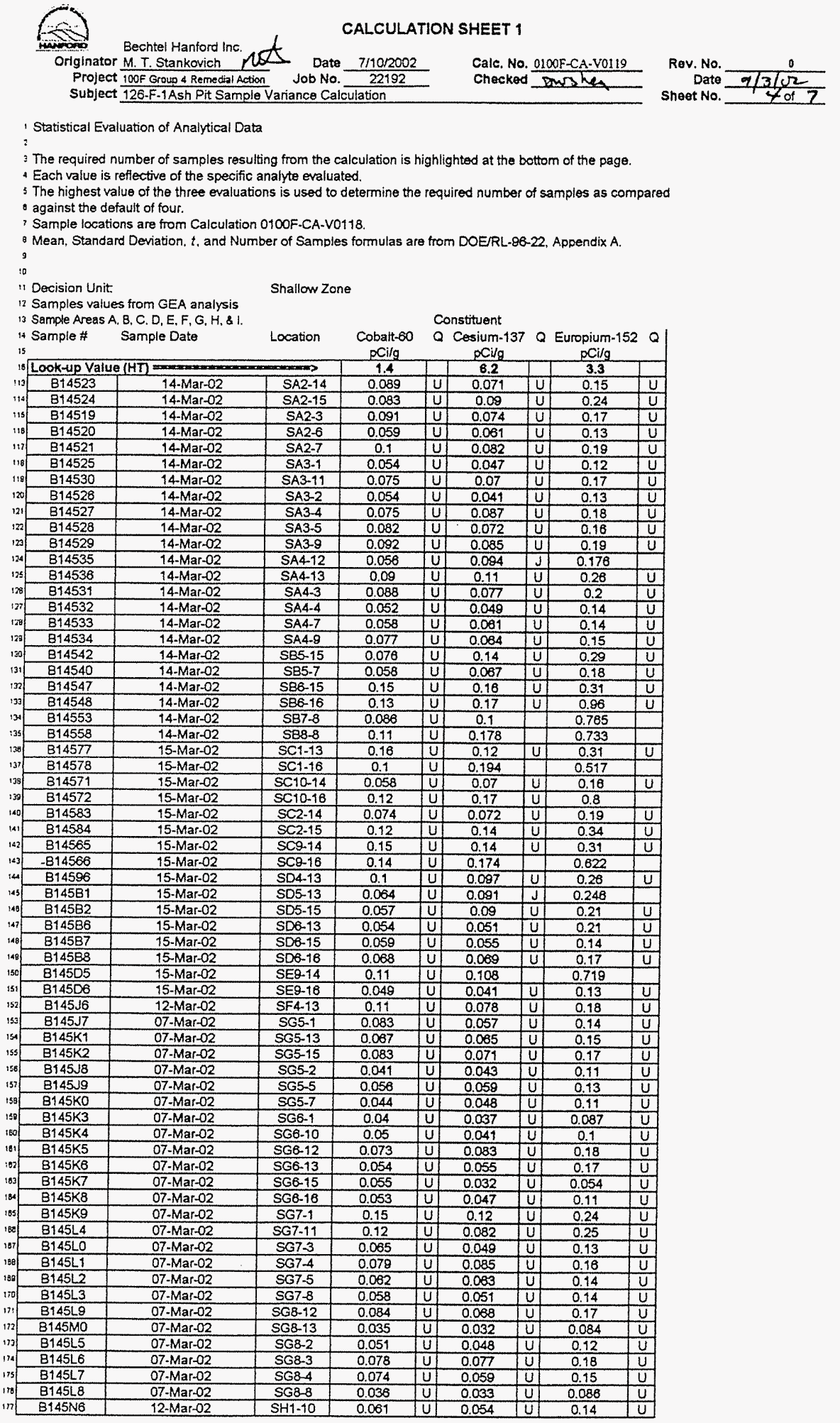




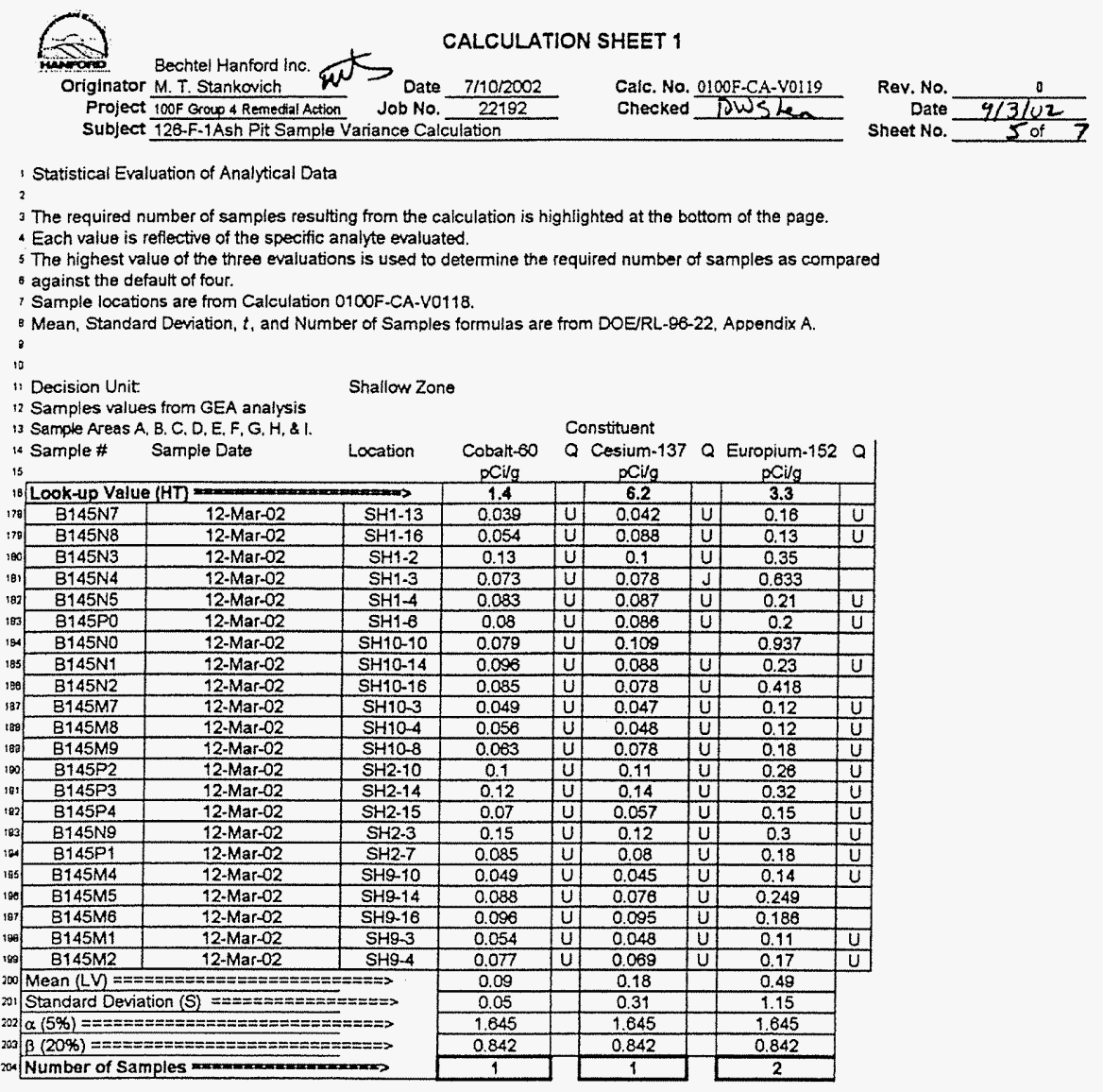




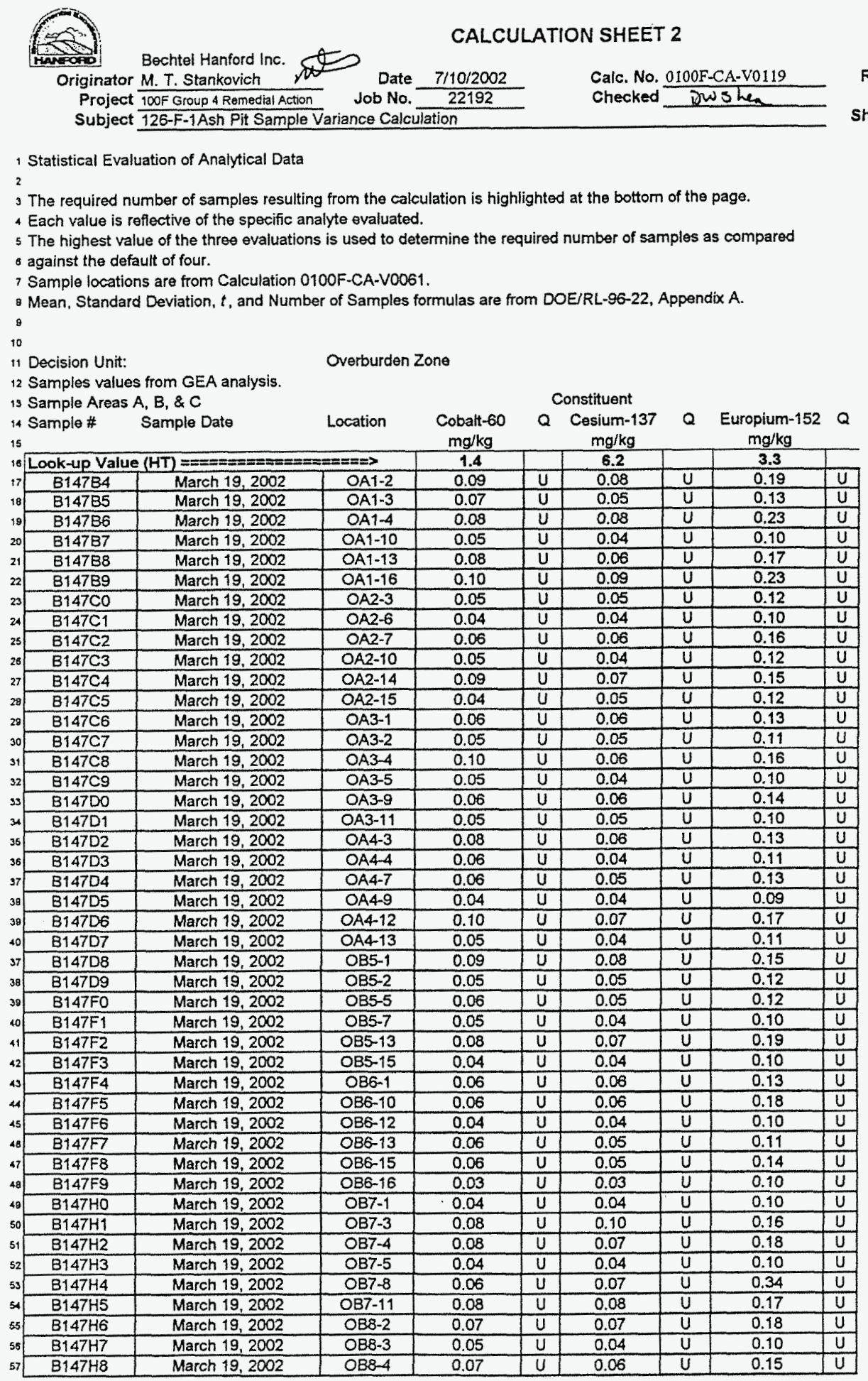




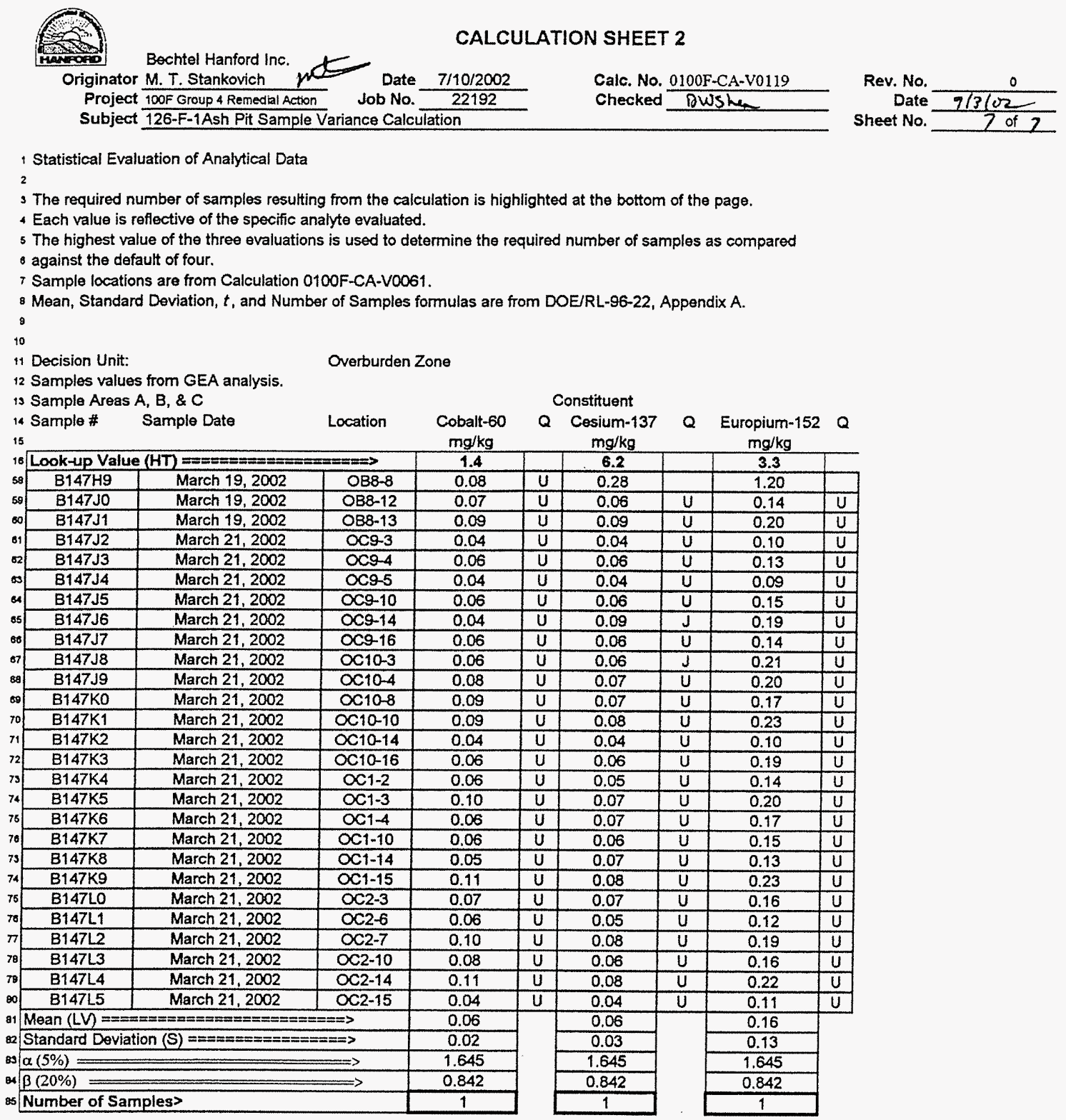




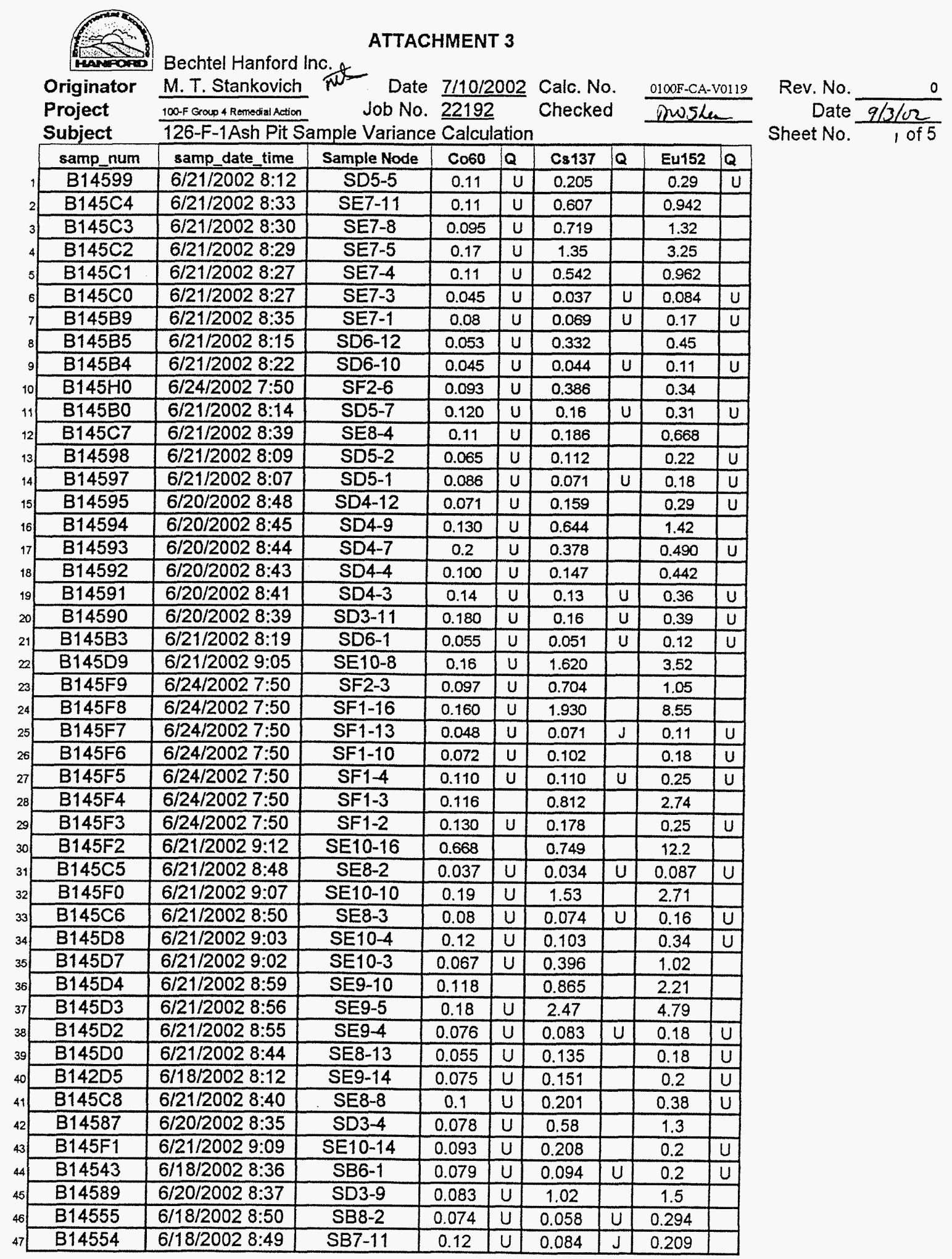




\begin{tabular}{|c|c|c|c|c|c|c|c|c|c|}
\hline & $\begin{array}{l}\text { Originator } \\
\text { Project } \\
\text { Subject }\end{array}$ & $\begin{array}{l}\text { Bechtel Hanford } \\
\text { M. T. Stankovich } \\
100-\text { F Grop } 4 \text { Remodal Aetion } \\
126-F-1 \text { Ash Pit S }\end{array}$ & $\begin{array}{l}\text { Date } \\
\text { Job No. } \\
\text { aple Varianc }\end{array}$ & $\frac{\frac{7 / 10 / 2}{22192}}{\text { Calcula }}$ & & $\begin{array}{l}\text { Calc. No } \\
\text { Checke }\end{array}$ & & $\begin{array}{l}\text { 0100F-CA-V } \\
\text { Plousher }\end{array}$ & \\
\hline & samp_num & samp_date_time & Sample Node & Co60 & $\mathbf{Q}$ & Cs137 & Q & Eu152 & $Q$ \\
\hline & $\mathrm{B} 14552$ & $6 / 18 / 20028: 46$ & SB7-5 & 0.039 & $U$ & 0.069 & $J$ & 0.087 & $U$ \\
\hline & B14551 & $6 / 18 / 20028: 45$ & SB7-4 & 0.057 & $U$ & 0.075 & $\mathrm{~J}$ & 0.12 & $\mathrm{U}$ \\
\hline 5 & $\mathrm{B14550}$ & $6 / 18 / 20028: 44$ & SB7-3 & 0.098 & $U$ & 0.11 & $U$ & 0.513 & \\
\hline & B14549 & $6 / 18 / 20028: 43$ & SB7-1 & 0.110 & $U$ & 0.1 & $u$ & 0.23 & $u$ \\
\hline & B14546 & $6 / 18 / 20028: 41$ & SB6-13 & 0.059 & $u$ & 0.085 & $u$ & 0.14 & $\mathrm{U}$ \\
\hline & $\mathrm{B} 14557$ & $6 / 18 / 20028: 52$ & SB8-4 & 0.094 & U & 0.1 & $u$ & 0.22 & $U$ \\
\hline 54 & 814544 & $6 / 18 / 20028: 37$ & SB6-10 & 0.072 & $U$ & 0.072 & J & 0.14 & $U$ \\
\hline & $B 14559$ & $6 / 18 / 20028: 53$ & SB8-12 & 0.12 & $U$ & 0.100 & $u$ & 0.26 & $U$ \\
\hline & $\mathrm{B} 14541$ & $6 / 18 / 20028: 33$ & SB5-13 & 0.11 & $\mathrm{u}$ & 0.285 & & 0.20 & $u$ \\
\hline & $\mathrm{B14539}$ & $6 / 18 / 20028: 31$ & SB5-5 & 0.047 & $\mathrm{U}$ & 0.051 & $u$ & 0.13 & $u$ \\
\hline & B14538 & $6 / 18 / 20028: 29$ & SB5-2 & 0.064 & $U$ & 0.050 & $\mathrm{~J}$ & 0.12 & $U$ \\
\hline & B14537 & $6 / 18 / 20028: 28$ & SB5-13 & 0.088 & $U$ & 0.063 & $U$ & 0.14 & $U$ \\
\hline & B14518 & $6 / 18 / 20028: 25$ & SA1-16 & 0.053 & U & 0.128 & & 0.236 & \\
\hline & B14517 & $6 / 18 / 20028: 22$ & SA1-13 & 0.081 & $U$ & 0.332 & & 0.215 & \\
\hline & $\mathrm{B} 14516$ & $6 / 18 / 20028: 19$ & SA1-10 & 0.1 & $U$ & 078 & $U$ & 0.17 & $U$ \\
\hline & 814515 & $6 / 18 / 20028: 18$ & SA1-4 & 0.068 & $\mathrm{U}$ & 0.055 & $U$ & 0.13 & $\bar{U}$ \\
\hline & 814514 & $6 / 18 / 20028: 15$ & SA1-3 & 0.059 & $\bar{U}$ & 0.096 & J & 0.17 & $\bar{U}$ \\
\hline & B14545 & $6 / 18 / 20028: 39$ & SB6-12 & 0.14 & 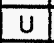 & 0.150 & $\mathrm{U}$ & 0.260 & U \\
\hline & B14573 & $6 / 20 / 20028: 19$ & SC1-2 & 0.120 & $\bar{U}$ & 0.086 & $\bar{U}$ & 0.22 & $\bar{U}$ \\
\hline & $\mathrm{B} 145 \mathrm{D1}$ & $6 / 21 / 20028: 51$ & SE9-3 & 0.178 & & 0.816 & & 3.64 & \\
\hline & B14586 & $6 / 20 / 20028: 32$ & SD3-2 & 0.085 & $U$ & 0.078 & $U$ & 0.18 & $u$ \\
\hline & $B 14585$ & $6 / 20 / 20028: 31$ & SD3-1 & 0.11 & U & 0.095 & $u$ & 0.23 & U \\
\hline & $B 14582$ & $6 / 20 / 20028: 29$ & SC2-10 & 0.078 & $U$ & 0.142 & & 0.21 & $u$ \\
\hline & B14581 & $6 / 20 / 20028: 28$ & SC2-7 & 0.15 & $U$ & 0.420 & & 0.545 & \\
\hline & B14580 & $6 / 20 / 20028: 27$ & $\mathrm{SC2}-6$ & 0.130 & $u$ & 0.161 & & 0.439 & \\
\hline & B14579 & $6 / 20 / 20028: 26$ & SC2-3 & 0.120 & $u$ & 0.130 & $u$ & 0.31 & $u$ \\
\hline & B14576 & $6 / 20 / 20028: 23$ & SC1-10 & 0.130 & 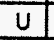 & 0.651 & & 0.985 & \\
\hline & B14556 & $6 / 18 / 20028: 51$ & SB8-3 & 0.080 & $U$ & 0.162 & & $\overline{0.419}$ & \\
\hline & $\mathrm{B14574}$ & $6 / 20 / 20028: 21$ & SC1-3 & 0.069 & $\bar{U}$ & 0.060 & $u$ & 0.17 & $u$ \\
\hline & $\mathrm{B} 14588$ & $6 / 20 / 20028: 36$ & SD3-5 & 0.140 & $U$ & 0.378 & & 0.72 & \\
\hline & $B 14570$ & $6 / 20 / 20028: 17$ & SC10-10 & 0.140 & $u$ & 0.160 & $u$ & 0.31 & $u$ \\
\hline & $B 14569$ & $6 / 20 / 20028: 16$ & SC10-8 & 0.059 & $U$ & 0.455 & & 0.648 & \\
\hline & 814568 & $6 / 20 / 20028: 14$ & SC10-4 & 0.15 & U & 0.13 & $U$ & 0.45 & $U$ \\
\hline & 814567 & $6 / 20 / 20028: 13$ & SC10-3 & 0.254 & & 0.658 & & 2.44 & \\
\hline & B14564 & $6 / 20 / 20028: 10$ & Sc9-10 & 0.07 & $U$ & 0.202 & & 0.18 & $\mathrm{U}$ \\
\hline & B14563 & $6 / 20 / 2$ & SC9-5 & 0.12 & U & 0.11 & $\mathrm{U}$ & 0.3 & $\mathrm{U}$ \\
\hline & $B 14562$ & $6 / 20 / 20028: 07$ & SC9-4 & 0.083 & $U$ & 0.16 & $U$ & 0.663 & \\
\hline & $\mathrm{B} 14561$ & $6 / 20 / 20028: 05$ & SC9-3 & 0.12 & U & 0.238 & & 0.957 & \\
\hline & $\mathrm{B14560}$ & $6 / 18 / 20028: 55$ & SB8-13 & 0.09 & $\mathrm{U}$ & 0.304 & & 0.334 & \\
\hline & B14575 & $6 / 20 / 20028: 22$ & SC1-4 & 0.11 & $U$ & 0.11 & $\bar{U}$ & 0.28 & $\bar{U}$ \\
\hline & B145J0 & $6 / 24 / 20027: 50$ & SF3-11 & 0.057 & $U$ & 0.147 & & 0.511 & \\
\hline & $\mathrm{B} 145 \mathrm{HI}$ & $6 / 24 / 20027: 50$ & SF2-7 & 0.1 & $U$ & 0.148 & & 0.32 & $\bar{U}$ \\
\hline & $\mathrm{B} 145 \mathrm{M} 3$ & $6 / 24 / 20028: 52$ & $\mathrm{SH} 9-5$ & 0.053 & $U$ & 0.059 & $J$ & 0.523 & \\
\hline & B145J5 & $6 / 24 / 20027: 50$ & SF4-12 & 0.046 & U & 0.049 & U & 0.1 & $U$ \\
\hline & B145J4 & $6 / 24 / 20027: 50$ & SF4-9 & 0.063 & $U$ & 0.07 & U & 0.18 & U \\
\hline & B145J3 & $6 / 24 / 20027: 50$ & SF4-7 & 0.043 & $U$ & 0.044 & $U$ & 0.1 & $\mathrm{U}$ \\
\hline & B145C9 & $6 / 21 / 20028: 41$ & SE8-12 & 0.12 & $U$ & 0.6 & & 0.5 & $\bar{U}$ \\
\hline
\end{tabular}




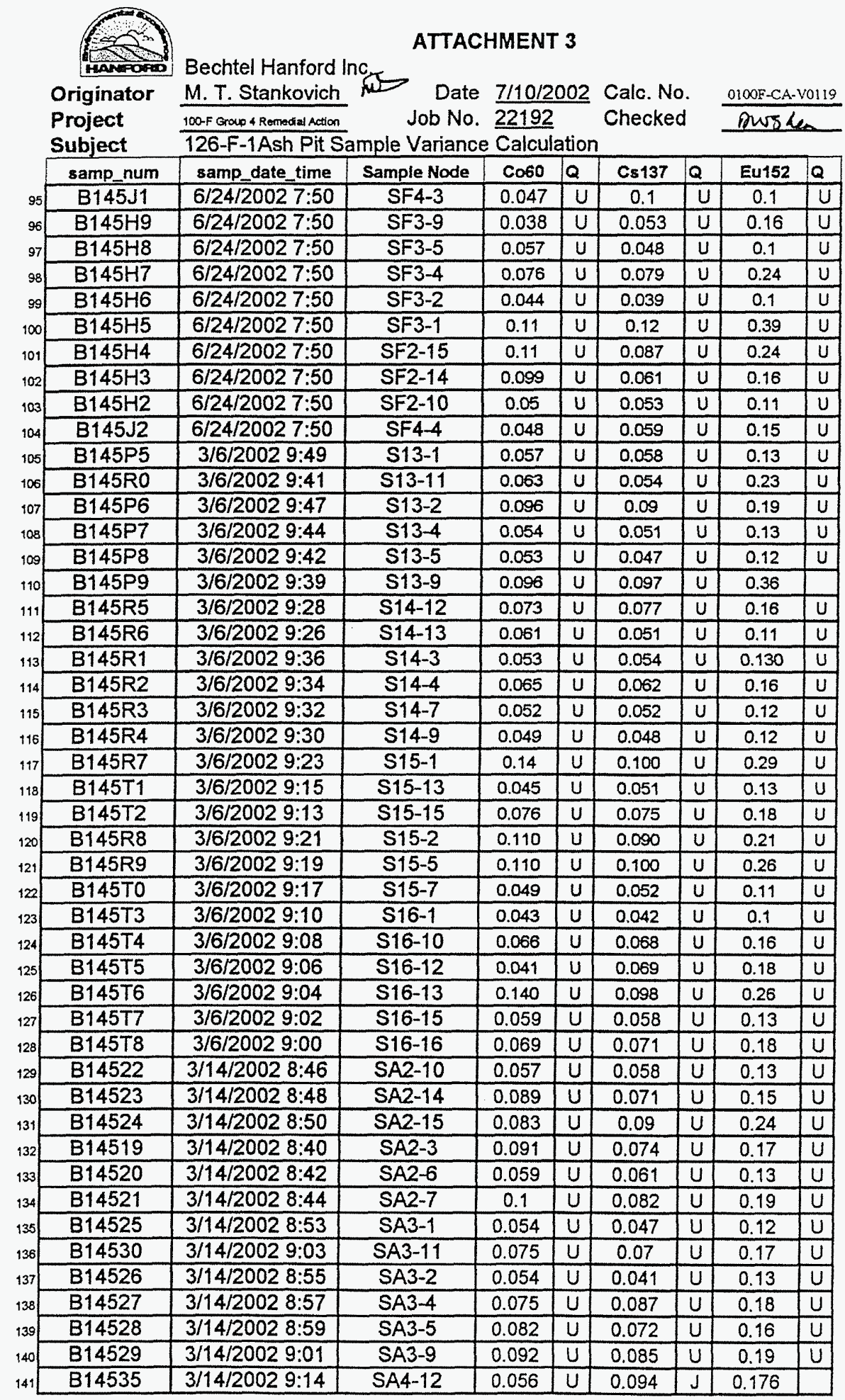




\begin{tabular}{|c|c|c|c|c|c|c|c|c|c|}
\hline & $\begin{array}{l}\text { Originator } \\
\text { Project } \\
\text { Subject }\end{array}$ & $\begin{array}{l}\text { Bechtel Hanford I } \\
\text { M. T. Stankovich } \\
\text { 100-F Grow 4 Remediat Action } \\
126-\text { - Ash Pit S }\end{array}$ & $\begin{array}{l}\text { Date } \\
\text { Job No. } \\
\text { imple Varianc }\end{array}$ & $\frac{\frac{7 / 10 / 20}{22192}}{\text { Calcula }}$ & & $\begin{array}{l}\text { Calc. No } \\
\text { Checkec }\end{array}$ & & $\begin{array}{l}0100 \mathrm{~F}-\mathrm{CA}-\mathrm{V} \\
\text { Dows }\end{array}$ & vol19 \\
\hline & samp num & samp_date_time & Sample Node & Co60 & $Q$ & Cs137 & a & Eu152 & \\
\hline & 814536 & $3 / 14 / 20029: 16$ & SA4-13 & 0.09 & $u$ & 0.11 & U & 0.26 & \\
\hline & B14531 & 3/14/2002 9:06 & SA4-3 & 0.088 & $U$ & 0.077 & $U$ & 0.2 & U \\
\hline & B14532 & $3 / 14 / 20029: 08$ & SA4-4 & 0.052 & $U$ & 0.049 & $U$ & 0.14 & $U$ \\
\hline & B14533 & $3 / 14 / 20029: 10$ & SA4-7 & 0.058 & $U$ & 0.061 & $u$ & 0.14 & $\bar{U}$ \\
\hline & 814534 & $3 / 14 / 20029: 12$ & SA4-9 & 0.077 & $U$ & 0.064 & $u$ & 0.15 & $u$ \\
\hline & B14542 & $3 / 14 / 20029: 21$ & SB5-15 & 0.076 & $U$ & 0.14 & $u$ & 0.29 & $u$ \\
\hline & $\mathrm{B} 14540$ & $9: 19$ & SB5-7 & 0.058 & $u$ & 0.067 & $u$ & 0.18 & $u$ \\
\hline & B14547 & $9: 23$ & SB6-15 & 0.15 & $u$ & 0.16 & $u$ & & $\bar{u}$ \\
\hline & B14548 & $3 / 14 / 20029: 25$ & SB6-16 & 0.13 & $u$ & 0.170 & $u$ & 0.96 & $u$ \\
\hline & 814553 & $9: 27$ & SB7-8 & .09 & $u$ & 0.100 & & 0.765 & \\
\hline & B14558 & $9: 29$ & SB8-8 & & $u$ & & & 0.73 & \\
\hline & B14577 & $8: 38$ & SC1-13 & 0.16 & $u$ & 12 & $u$ & 31 & $u$ \\
\hline & B14578 & $3 / 1$ & SC1-16 & 0.1 & $u$ & 0.194 & & .52 & \\
\hline & B14571 & $8: 34$ & SC10-14 & 0.058 & $u$ & 0.07 & u & 16 & $u$ \\
\hline & B14572 & $3 / 15$ & SC10 & 0.12 & $U$ & 0.170 & $u$ & 0.8 & \\
\hline & B14583 & & SC2-14 & 0.074 & $u$ & 0.072 & $u$ & .19 & $u$ \\
\hline & B14584 & & SC2-15 & 0.12 & $u$ & .14 & $u$ & 34 & $\bar{u}$ \\
\hline & B14565 & & SC9-14 & 150 & $u$ & 14 & $u$ & 31 & $u$ \\
\hline & B14566 & & SC9-16 & 0.140 & $u$ & 0.174 & & 0.62 & \\
\hline & B14596 & $: 47$ & SD4- & 0.1 & $u$ & 0.097 & $u$ & 0.260 & $u$ \\
\hline & B145B1 & & SD5- & 0.08 & $u$ & & 7 & 0.246 & \\
\hline & B145B2 & & SD5- & 0.057 & U & 0.09 & $\mathrm{u}$ & .21 & $u$ \\
\hline & $\mathrm{B} 145 \mathrm{~B} 6$ & $3 / 1 !$ & SD6-1 & 0.054 & u & 051 & U & 21 & U \\
\hline & B145B7 & $3 / 1$ & SD6-15 & 0.05 & U & 0.055 & $u$ & & $u$ \\
\hline & B145B8 & & SD6-16 & 0.068 & U & .069 & $u$ & 0.17 & $\bar{u}$ \\
\hline & B145D5 & & SE9-14 & 0.11 & $u$ & 0.108 & & 0.719 & \\
\hline & $B 145[$ & & SE9-16 & 9 & $u$ & 41 & $u$ & 0.13 & $u$ \\
\hline & B145J6 & $3 / 1$ & SF4-13 & 0.110 & $u$ & 0.078 & $u$ & 0.18 & U \\
\hline & B145J7 & & SG5-1 & 0.083 & $u$ & 0.057 & $u$ & 0.14 & 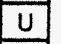 \\
\hline & B145K1 & & SG5-13 & 0.067 & $u$ & 0.065 & $u$ & 0.15 & $u$ \\
\hline & $\mathrm{B} 145 \mathrm{~K} 2$ & & SG5-15 & 0.083 & $u$ & 0.071 & $u$ & 0.17 & $\bar{U}$ \\
\hline & B145J8 & & SG5-2 & 0.041 & $u$ & 0.043 & $U$ & 0.11 & $\bar{u}$ \\
\hline & B145J9 & & SG5-5 & & $U$ & 0.059 & $u$ & & $U$ \\
\hline & B145KO & & SG5-7 & 0.044 & $U$ & .048 & $U$ & 0.11 & U \\
\hline & $\mathrm{B} 145 \mathrm{~K} 3$ & & SG6-1 & 0.04 & $U$ & 0.037 & $U$ & 0.087 & $\bar{U}$ \\
\hline & & & SG6-10 & & $U$ & & $U$ & 0.1 & $\mathrm{U}$ \\
\hline & B145K5 & & SG6-12 & & $U$ & & $U$ & 0.18 & $U$ \\
\hline & B145K6 & & SG6-13 & 0.054 & $U$ & 0.055 & $U$ & 0.17 & $\bar{U}$ \\
\hline & & & SG6-15 & & $U$ & 032 & $U$ & 0.054 & $U$ \\
\hline & B145K8 & & SG6-16 & 0.053 & $U$ & 0.047 & $U$ & 0.11 & $U$ \\
\hline & B145K9 & $3 / 7 / 20028: 53$ & SG7-1 & 0.15 & U & 0.12 & $U$ & 0.24 & $U$ \\
\hline & B145L4 & & SG7-11 & 0.12 & $U$ & 0.082 & $\mathrm{U}$ & 0.25 & 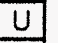 \\
\hline & B145LO & $8: 55$ & SG7-3 & & $\bar{U}$ & & $U$ & 0.13 & $\mathrm{U}$ \\
\hline & B145L1 & $3 / 7 / 20028: 57$ & SG7-4 & 0.079 & $\bar{U}$ & 0.085 & $U$ & 0.16 & $\mathrm{U}$ \\
\hline & B145L2 & $3 / 7 / 20028: 59$ & SG7-5 & 0.062 & $\underline{U}$ & 0.063 & $U$ & 0.14 & 4 \\
\hline & & & SG7-8 & 0.058 & $U$ & 0.051 & $U$ & 0.14 & $U$ \\
\hline & B145L9 & $3 / 7 / 20028: 48$ & SG8-12 & 0.084 & $U$ & 0.068 & $U$ & 0.17 & $U$ \\
\hline
\end{tabular}

Rev. No.

$$
\text { Date } \overline{9 / 3 / c 2}
$$

Sheet No. $\frac{9 / 3 / 0}{4 \text { of }}$ 


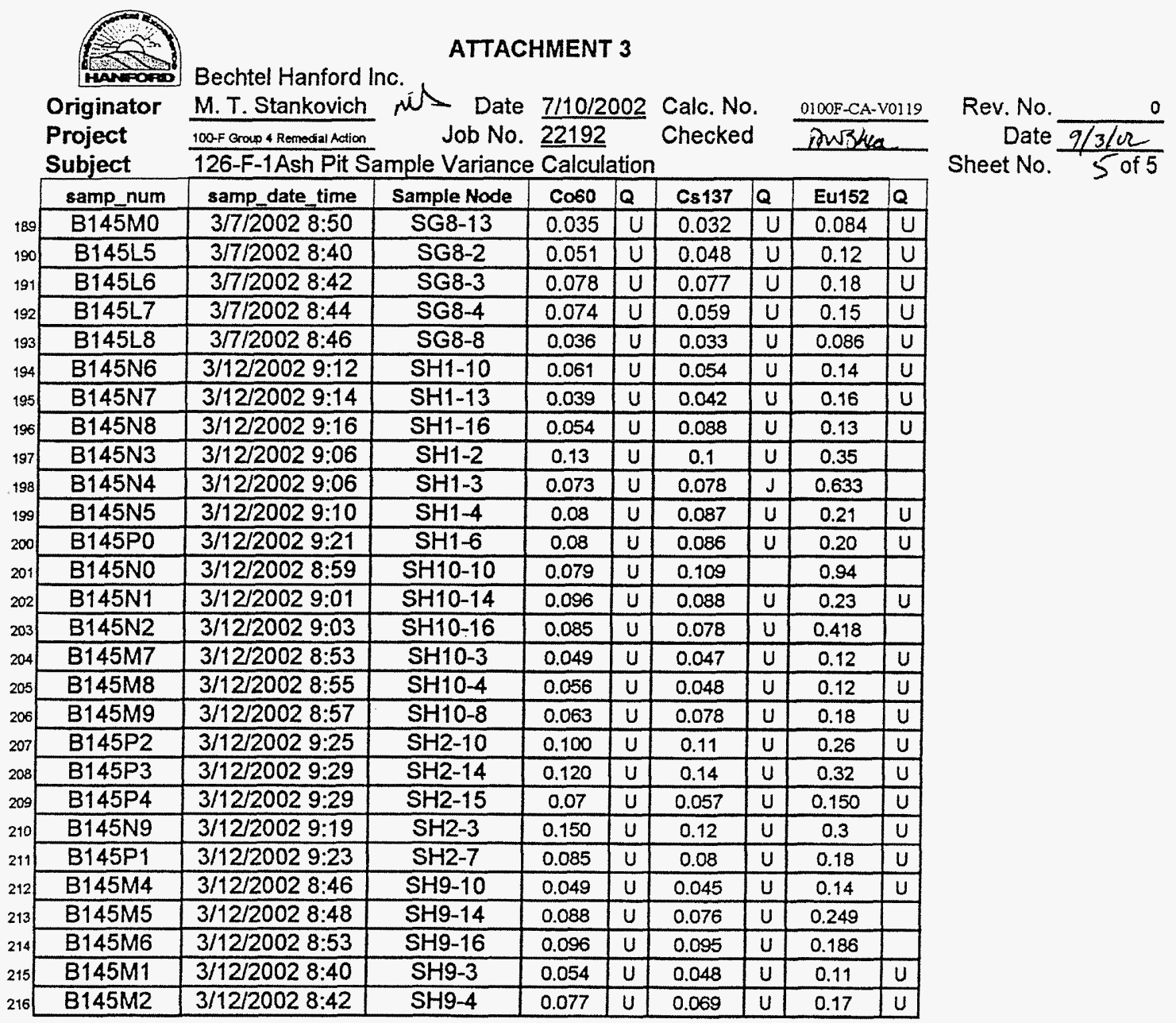




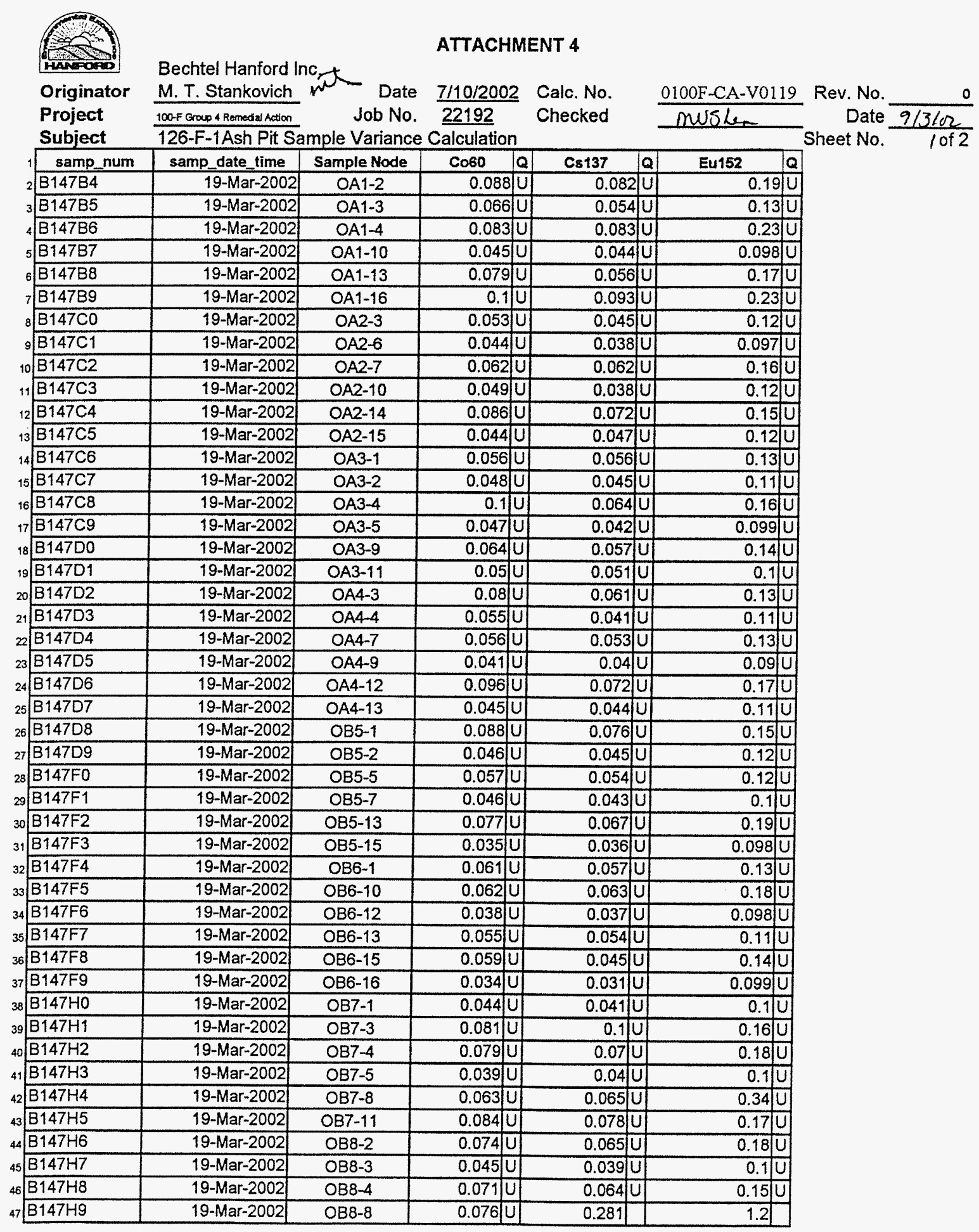




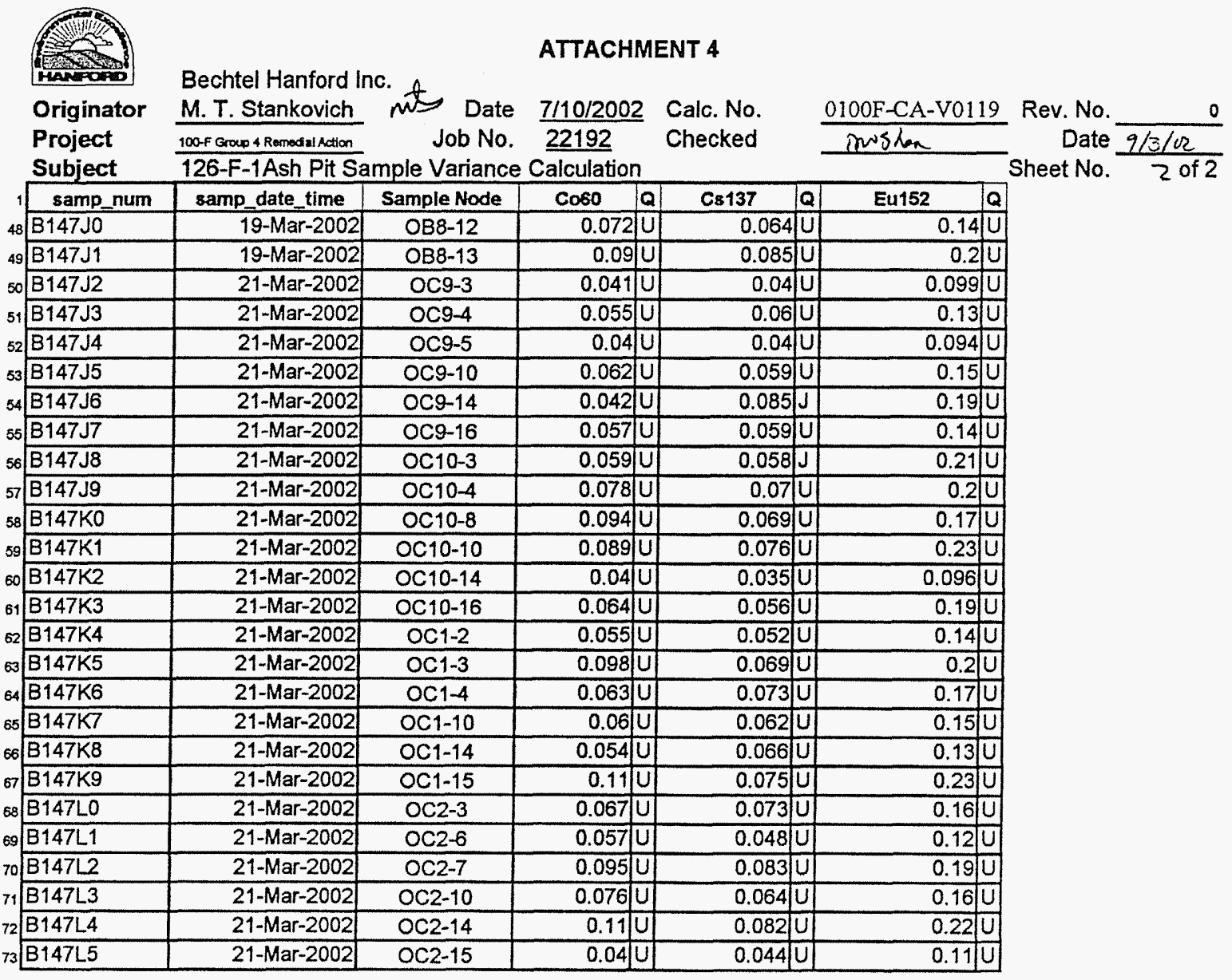


CALCULATION COVER SHEET

Project Title: 126-F-1 Overburden Sample Design Job No. 22192

Area 100-F

Discipline_Environmental Engineering $\quad{ }^{*}$ Calc. No. 0100F-CA-V0061

Subject 126-F-1 Overburden Sampling Design

Computer Program Excel

Program No. Office 97 Version

Committed Calculation

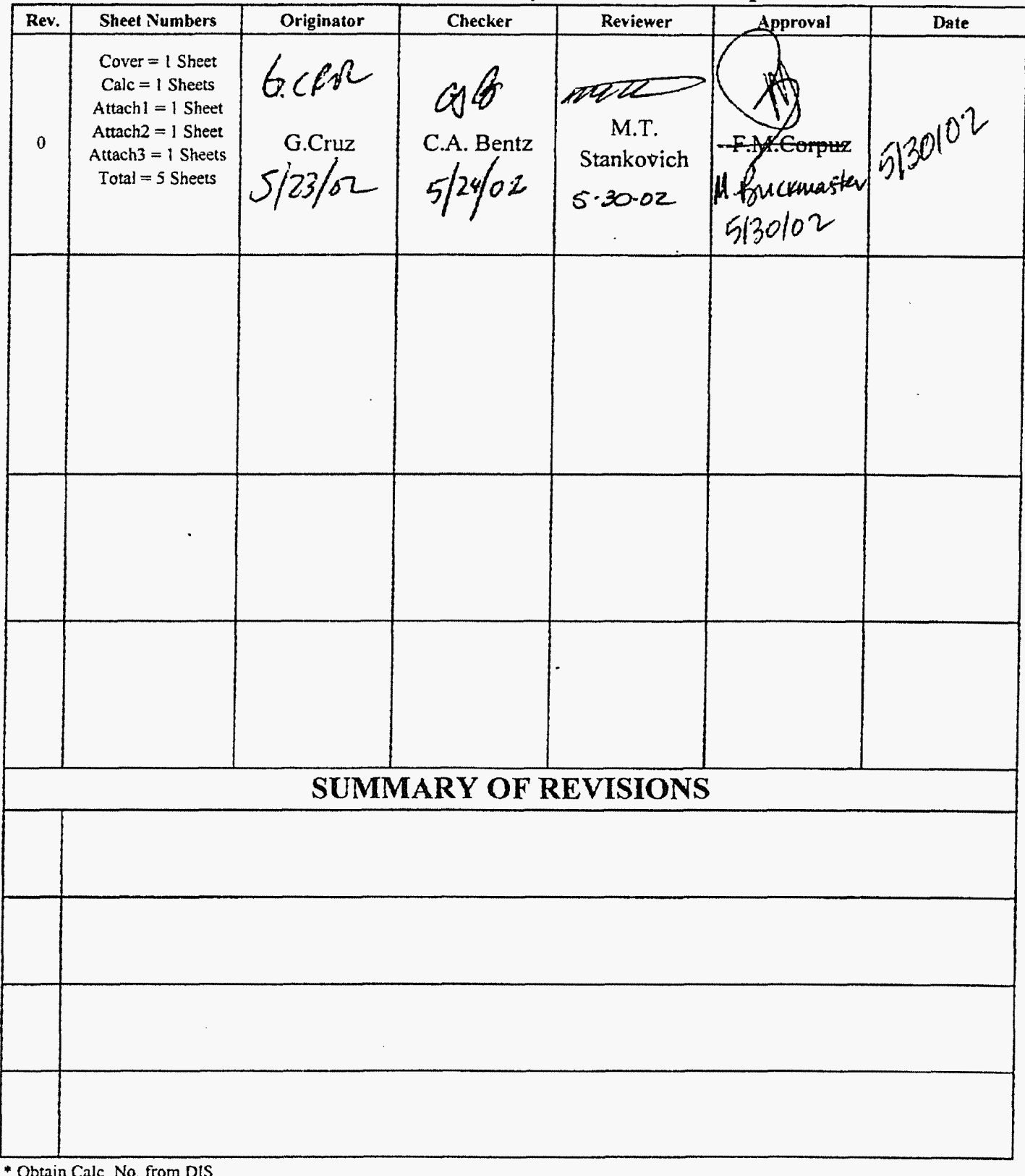

* Obtain Calc. No. from DIS 
Project 126-F-1 Overburden Sample Design Job No. 22192 Checked OS/3 Date $5 / 24 / 02$ Subject 126-F-1 Overburden Sampling Design Sheet No.

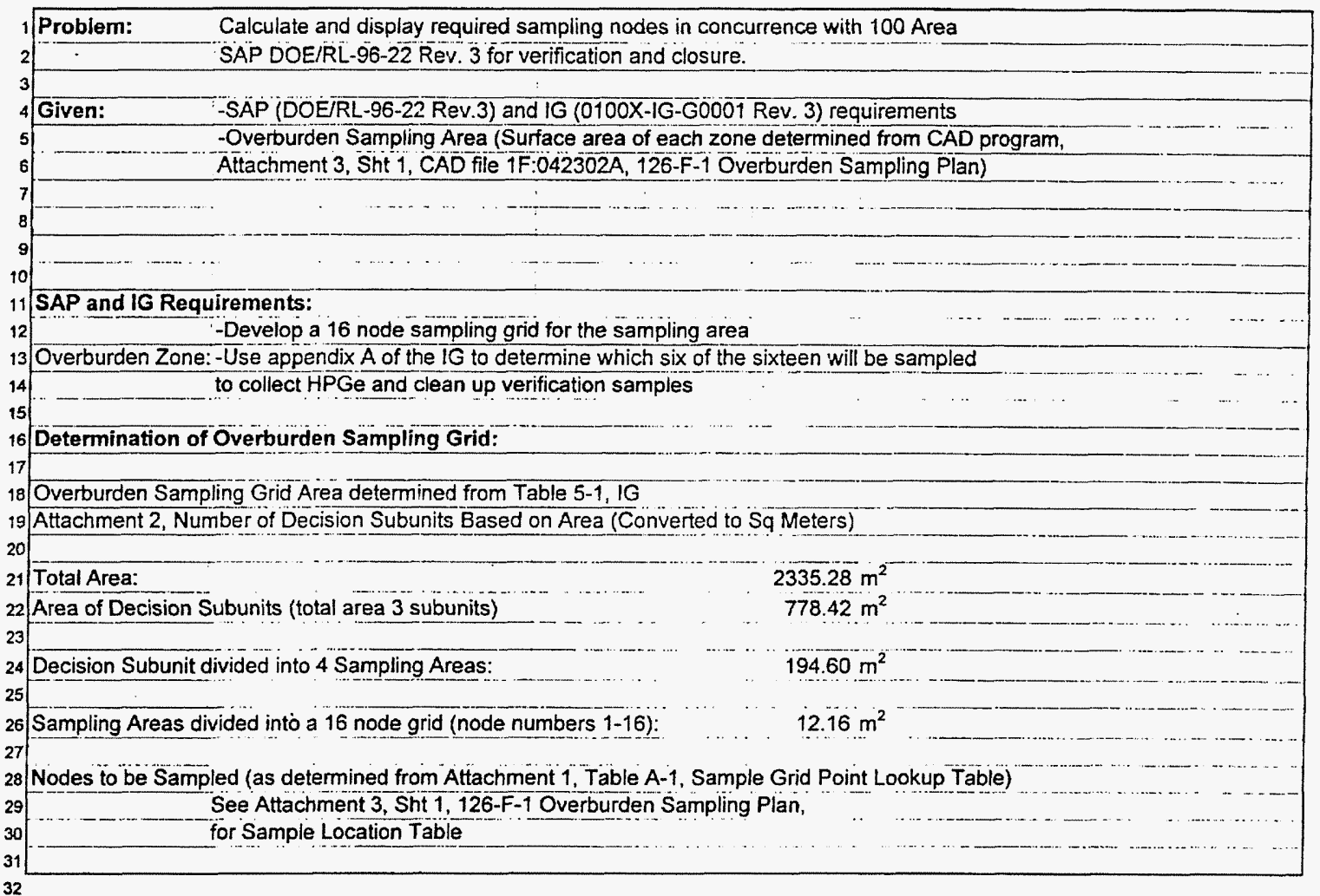


<smiles>CCCCCC(C)CCC</smiles>

Originator Gerardo Cruz Date 04/23/02 Calc. No. 0100F-CA-V0061 Rev. No. 0

Project 126-F-1 Overburden Sample Design Job No. 22192 Checked Q8 G Date $5 / 24 / 02$ Subject 126-F-1 Overburden Sampling Design Sheet No.

2 ATTACHMENT 1

3

${ }_{4}$ Sample Grid Point Lookup Table.

$$
5
$$

.

\begin{tabular}{|c|c|c|c|c|c|c|c|c|c|c|}
\hline Default Plan & $\begin{array}{c}\text { Sampling } \\
\text { Area } 1\end{array}$ & $\begin{array}{c}\text { Sampling } \\
\text { Area } 2\end{array}$ & $\begin{array}{c}\text { Sampling } \\
\text { Area } 3\end{array}$ & $\begin{array}{l}\text { Sampling } \\
\text { Area } 4\end{array}$ & $\begin{array}{c}\text { Sampling } \\
\text { Area } 5\end{array}$ & $\begin{array}{l}\text { Sampling } \\
\text { Area } 6\end{array}$ & $\begin{array}{c}\text { Sampling } \\
\text { Area } 7\end{array}$ & $\begin{array}{c}\text { Sampling } \\
\text { Area } 8\end{array}$ & $\begin{array}{c}\text { Sampling } \\
\text { Area } 9\end{array}$ & $\begin{array}{c}\text { Sampling } \\
\text { Area } 10\end{array}$ \\
\hline HPGe/Closeout & 3 & 6 & 1 & 4 & 5 & 1 & 3 & 3 & 4 & 16 \\
\hline HPGe/Closeout & 4 & 7 & 11 & 3 & 15 & 15 & 5 & 13 & 10 & 10 \\
\hline HPGe/Closeout & 16 & 3 & 2 & 7 & 7 & 10 & 11 & 4 & 3 & 14 \\
\hline HPGe/Closeout & 10 & 15 & 4 & 12 & 1 & 13 & 4 & 8 & 16 & 4 \\
\hline HPGe & 2 & 14 & 5 & 9 & 13 & 12 & 8 & 2 & 14 & 8 \\
\hline HPGe & 13 & 10 & 9 & 13 & 2 & 16 & 1 & 12 & 5 & 3 \\
\hline Not Sampling & 6 & 1 & 10 & 8 & 14 & 4 & 16 & 5 & 8 & 6 \\
\hline Not Sampling & 1 & 9 & 13 & 1 & 10 & 5 & 12 & 1 & 1 & 15 \\
\hline Not Sampling & 9 & 12 & 7 & 5 & 6 & 2 & 6 & 7 & 15 & 9 \\
\hline Not Sampling & 15 & 16 & 15 & 14 & 16 & 6 & 2 & 15 & 11 & 1 \\
\hline Not Sampling & 8 & 13 & 8 & 10 & 12 & 11 & 13 & 14 & 2 & 12 \\
\hline Not Sampling & 5 & 2 & 3 & 11 & 4 & 3 & 9 & 10 & 7 & 11 \\
\hline Not Sampling & 7 & 11 & 14 & 15 & 11 & 14 & 14 & 6 & 13 & 2 \\
\hline Not Sampling & 11 & 4 & 6 & 2 & 9 & 7 & 7 & 11 & 9 & 7 \\
\hline Not Sampling & 12 & 8 & 16 & 16 & 3 & 8 & 15 & 9 & 6 & 13 \\
\hline Not Sampling & $\cdot 14$ & 5 & 12 & 6 & 8 & 9 & 10 & 16 & 12 & 5 \\
\hline
\end{tabular}

24 ** Note: Grid nodes for each sampling area in each waste site should be numbered consistently, e.g., begin numbering ${ }_{25}$ the nodes in the northwesternmost node. Then number consecutively left to right as shown in Fig. 5-1 of this IG 


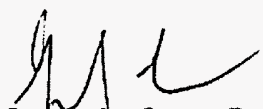

Originator Gefardo Cruz Date 04/23/02 Calc. No.0100F-CA-V0061 Rev. No. 0

Project 126-F-1 Overburden Sample Design Job No. 22192 Checked $Q B$

Subject 126-F-1 Overburden Sampling Design Sheet No.

\section{ATTACHMENT 2}

3

${ }_{4}$ Number of Decision Subunits Based on Area.

5

\begin{tabular}{|c|c|c|}
\hline 7 & Area of Primary Decision Unit (m2) & Number of Subunits \\
\hline 8 & $<1,394$ & 1 \\
\hline 9 & $>1,394$ to $<2,326$ & 2 \\
\hline 0 & $>2,326$ to $<3,256$ & 3 \\
\hline & $>3,256$ to $<4,186$ & 4 \\
\hline 2 & $>4,186$ to $<9,303$ & 2 \\
\hline & $>9,303$ to $<13,024$ & 3 \\
\hline & $>13,024$ to $<16,745$ & 4 \\
\hline & $>16,745$ to $<20,466$ & 5 \\
\hline & $>20,466$ & ROUNDa (Area/3,720) \\
\hline
\end{tabular}


CVP-2002-00004

Rev. 1

C-20 


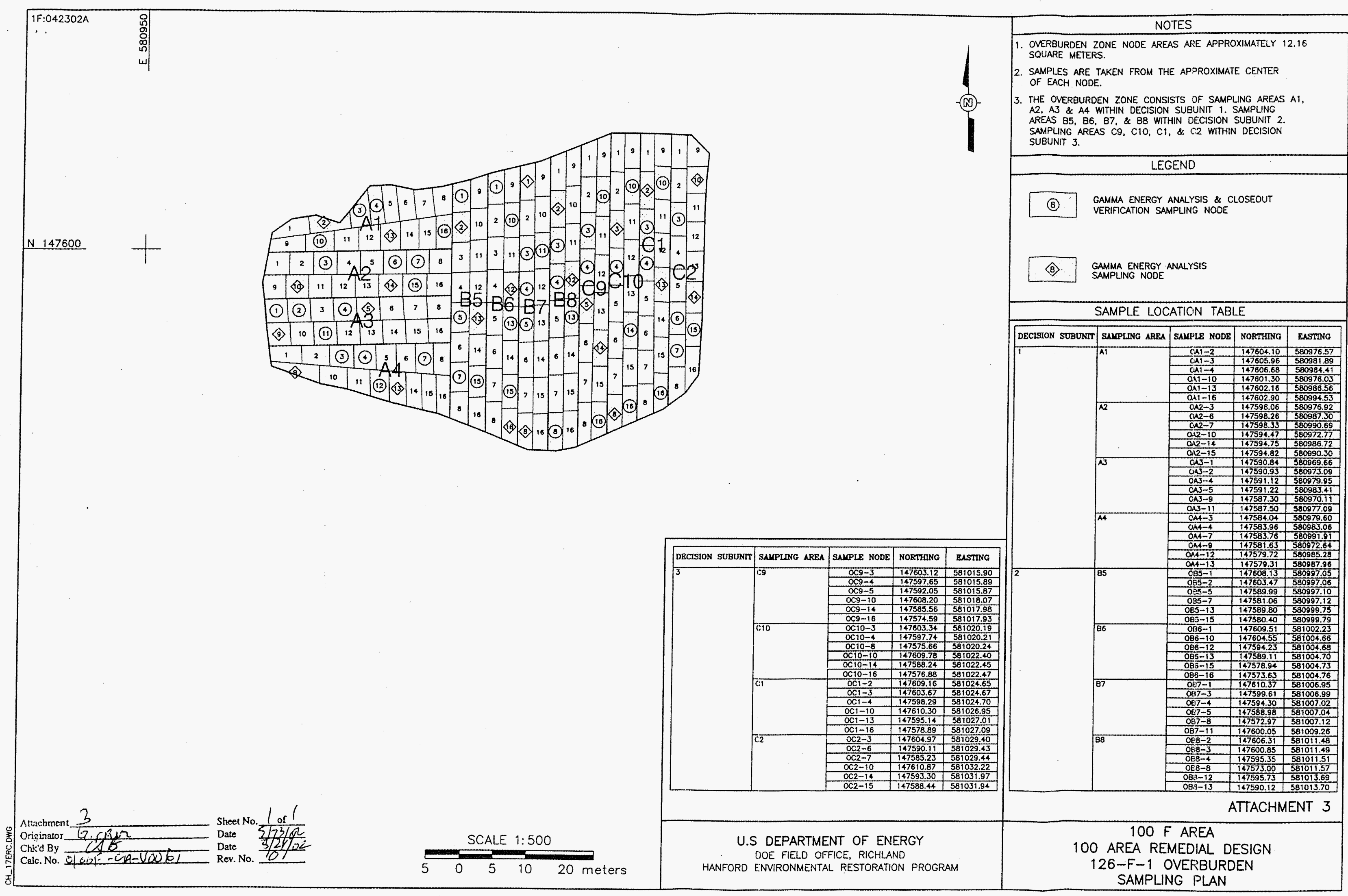




$$
\begin{array}{r}
\text { CVP-2002-00004 } \\
\text { Rev. } 1
\end{array}
$$

CALCULATION COVER SHEET

Project Title: 126-F-1 Ash Pit Shallow Zone Design Job No.

22192

Area 100-F

Discipline_Environmental Engineering_ $\quad *$ Call. No. 0100F-CA-V0118

Subject 126-F-1 Ash Pit Shallow Zone Sample Design

Computer Program Excel

Program No.

Office 97 Version

Committed Calculation \& Preliminary

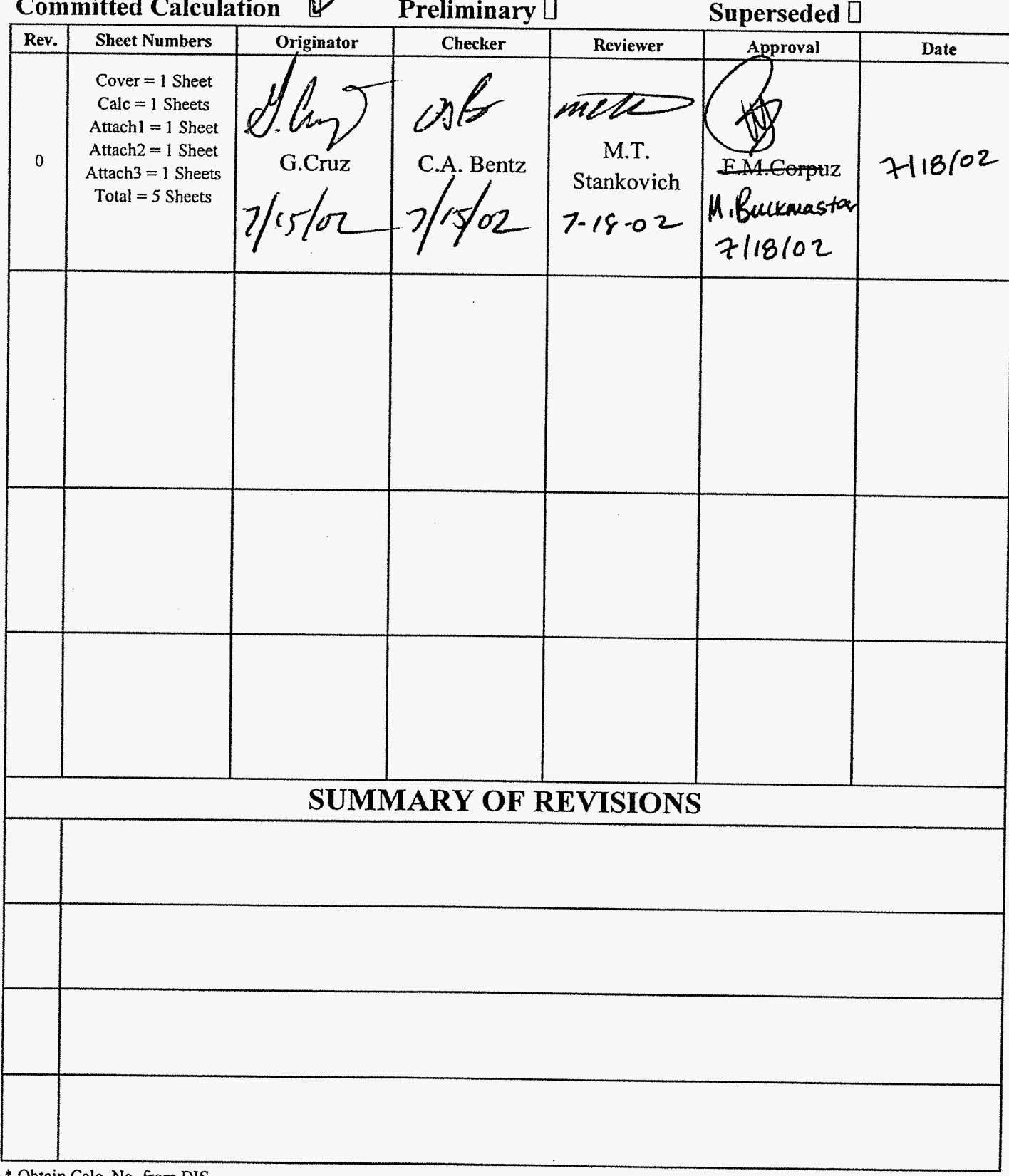

* Obtain Call. No. from DIS .

C-23 
Originator Gerardo Cruz Date 07/15/02 Calc. No. 0100F-CA-V0118 Rev. No. 0
Project 126-F-1 Ash Pit Shallow Zone Design Job No. 22192 Checked At\& Subject 126-F-1 Ash Pit Shallow Zone Sample Design Sheet No.

Subject 126-F-1Ash Pit Shallow Zone Sample Design Sheet No.

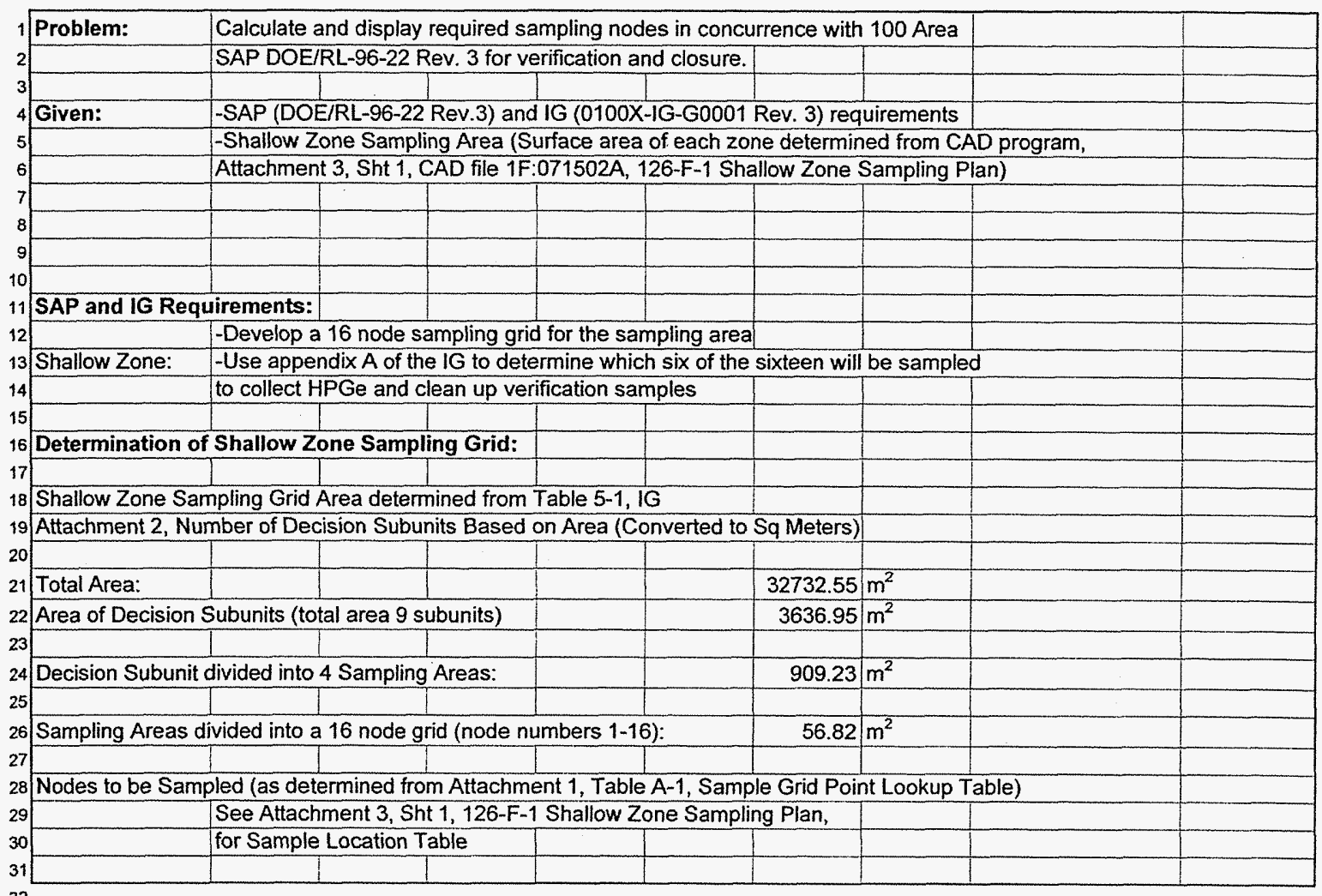


Originator Gerardo Cruz Date 07/15/02 Calc. No.0100F-CA-V0118 Rev. No. 0 Project 126-F-1 Ash Pit Shallow Zone Design Job No. 22192 Checked CO\& Date $7 / 15 / 02$ Subject 126-F-1 Ash Pit Shallow Zone Sample Design Sheet No.

1

2 ATTACHMENT 1

${ }_{4}$ Sample Grid Point Lookup Table.

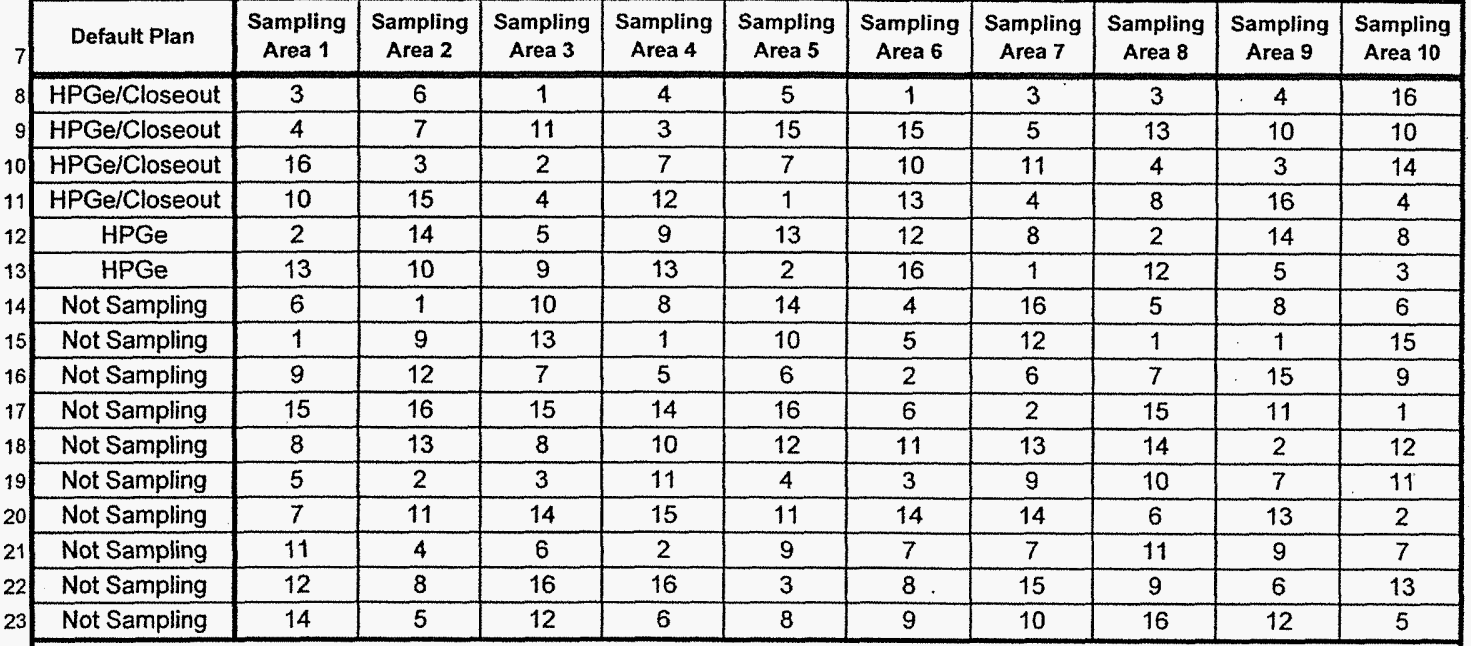

** Note: Grid nodes for each sampling area in each waste site should be numbered consistently, e.g., begin numbering ${ }_{25}$ the nodes in the northwesternmost node. Then number consecutively left to right as shown in Fig. 5-1 of this IG 


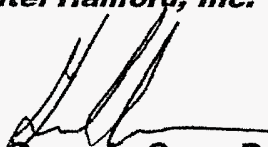

Originator Eerardo Cruz Date 07/15/02 Calc. No.0100F-CA-V0118 Rev. No. 0

Project 126-F-1 Ash Pit Shallow Zone Design Job No. 22192 Checked \& \& Date $7 / 15 / 02$ Subject 126-F-1 Ash Pit Shallow Zone Sample Design Sheet No.

1

2 ATTACHMENT 2

3

${ }_{4}$ Number of Decision Subunits Based on Area.

\begin{tabular}{|c|c|c|}
\hline 7 & Area of Primary Decision Unit (m2) & Number of Subunits \\
\hline 8 & $<1,394$ & 1 \\
\hline & $>1,394$ to $<2,326$ & 2 \\
\hline & $>2,326$ to $<3,256$ & 3 \\
\hline & $>3,256$ to $<4,186$ & 4 \\
\hline & $>4,186$ to $<9,303$ & 2 \\
\hline & $>9,303$ to $<13,024$ & 3 \\
\hline & $>13,024$ to $<16,745$ & 4 \\
\hline & $>16,745$ to $<20,466$ & 5 \\
\hline & $>20,466$ & ROUNDa (Area/3,720) \\
\hline
\end{tabular}

19

20

21

22

23

24

25

26

27

28

29

30

31

32

33

34

35

36

37

38

39

40

41

42 


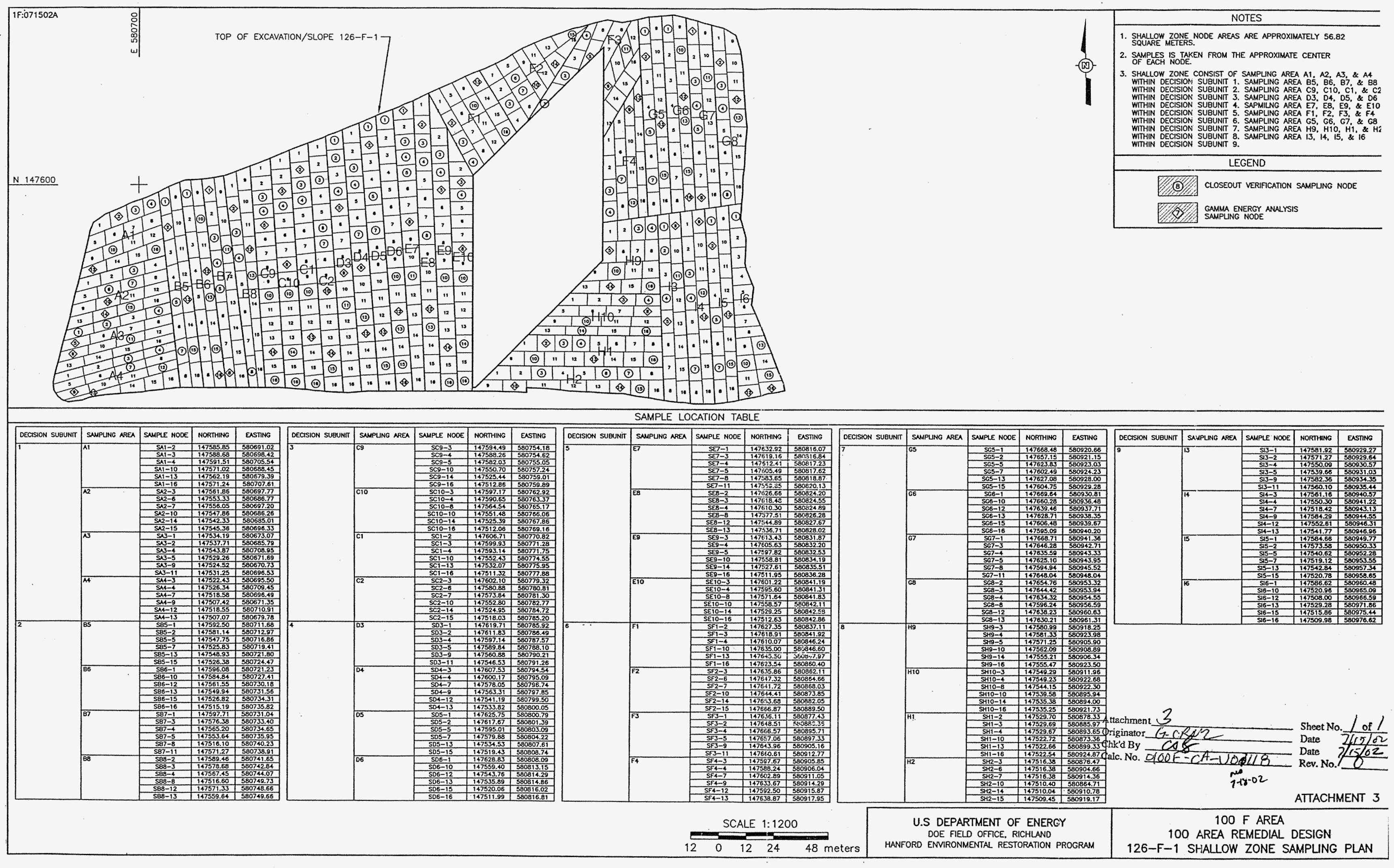




\section{CALCULATION COVER SHEET}

Project Title:

Area

Discipline

Subject

Computer Program
126-F-1 Northern Portion Cleanup Verification $100-\mathrm{F}$

Environmental

*alc No $0100 \mathrm{~F}-\mathrm{CA}-\mathrm{V} 0144$

126-F-1 Northern Portion Clcanup Verification $95 \%$ UCL Calculation

Excel

Program No. Excel 97
Committed Calculation $X$

Preliminary

Superseded

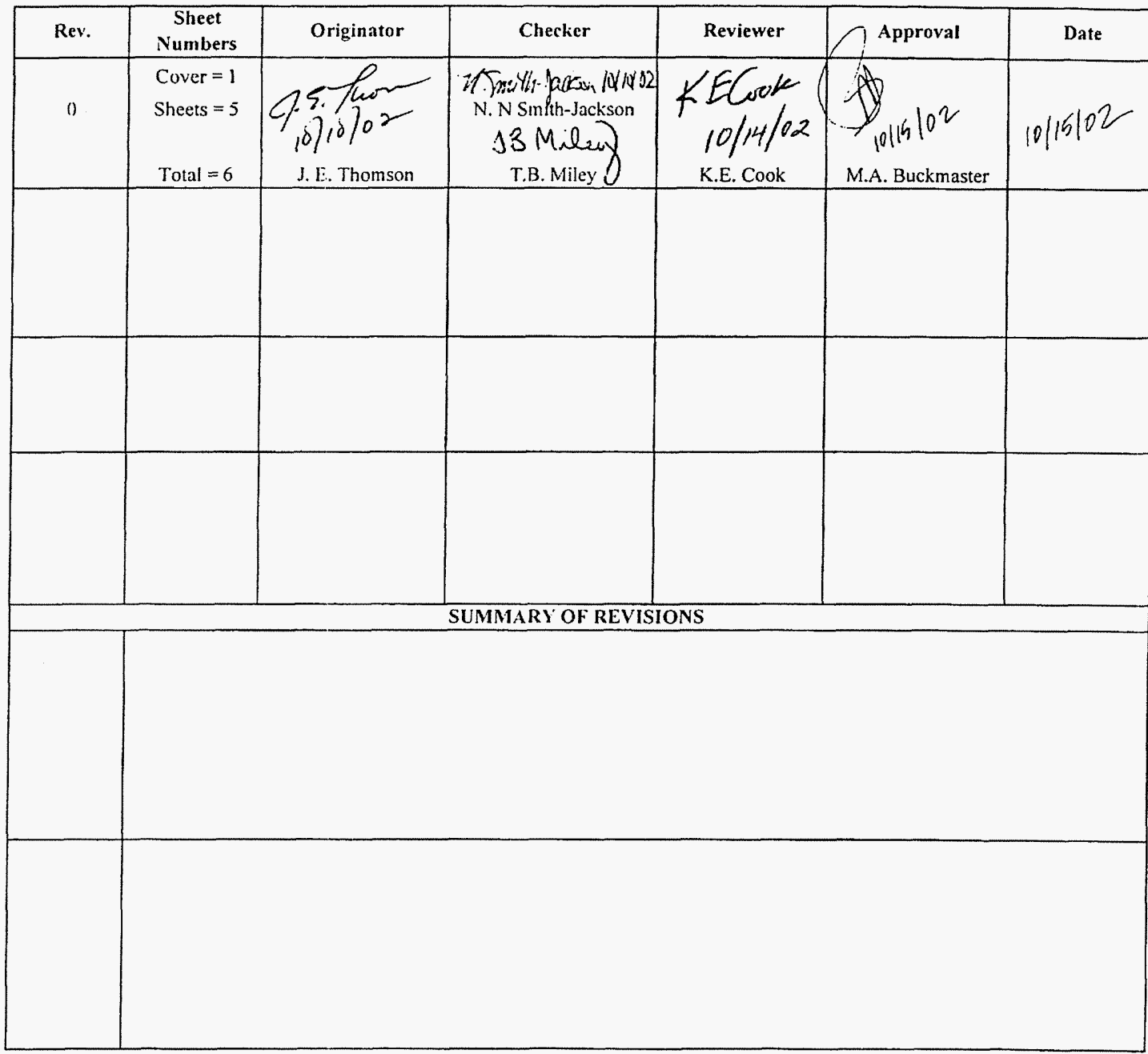

* Obtain calc no. from DIS 


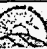

1 Purpose:

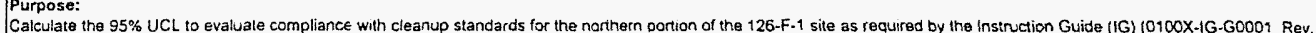
3). Also, calculale the catemogenic : isk, and priform the data quality assessment relative percent difference (RPD) for dupicates and splits of each contaminant of concern (COC)

Table of Contents:

Sheet 1 and 2 - Calculation Sheet Summary

Sheet 3 - Calculation Sheel Shalliow Zone Verfication

Sheet 5 - Calcutation Sneet Splt-Dup Analys:s

GiventReforences:

1 1) Sample Results

11 2) Lcokup values from Remedial Desıgn ReooruRemedial Action Work Plan (RDRIRAWP) (DOE-RL. 2001). Aill lookup values and RAGs are taken from

12 RDPURAWP uriless othenwise szectied

3 3) DOE-RL. 2001, 100 Area Remedial Action Sampling and Analysis Plan (SAP), DOEIRL-96-22. Rev. 3. U.S. Department of Energy, Richland

Operations Ofice Richland. Washington

4) DOE-RL, 2001. Remecial Design Report/Remedial Action Work Plan for the 100 Area, DOE/RL-96-17, Rev. 3, U.S. Department of

Energy, Richland Ooerations Otfice, Richland, Washington

BHI. 1999, Instruction Guide for the Remediation of the 100-8C-1. 100-OR-1, and 100-HR-1 Waste Sites, 0100X-1G-G0001. Rev. 3 ,

Bechtel Hantord. Inc. Richland Washingtor

Model Toxics Conirol Act. Wasnington Administrative Code-173-340, and Statıstical Guidance for Ecology Site Managers,

Ecology Pub. \#92-54. Washinglon Depanment of Ecology, Olympia. Washinglon

Ecology. 1993. Statistical Guidance for Ecology Ste Managers, Supploment S-6. Analyzing Sito or Background Data with

Below-Detectian Limit or Balow-PQL Valus (Censored Data Sets)

8) EPA 1994, WSEPA Contract Laboratory Program Nationat Functional Guidelines for inorganic Data Review. EPA 540/R-94/013.

Solution:

Calculation methosalogy is Jescribed in Ecology Pub. \#92-54, below, and in Attachment A-1 of the SAP. Use data trom attached worksheets to calculate the $95 \%$ upper confidence limit (UCL) for each analyte, carcinogenic risk, and RPD for each COC

Calculation Description:

The subject calculations were performed on data from soil verification samples from the northern portion of the 126-F-1 site. The data were entered into an EXCEL 97 spreadsheet

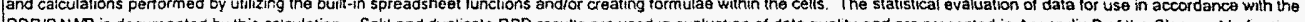
Package (CVP) for this ste.

Methodology:

The statstical value calculated to evaluate the elfectiveness of cleanup was the $95 \% \mathrm{UCL}$. For radronuclide dala, calculation of the statistics was done on the reported value. In cases where the laboratory soes not report a value telow the mintmal detectable aclivity (MDA), natf of the MDA is used in the calculation.

For the statistical evaluation of dupticate sample pairs, the samples are averaged betore being included in the data sot, after adjustments for censored data as described above.

Because there are no nonradionuclice COCs identfifed for the 126-F-1 site. the MTCA 3-part test. estimated hazard quotiont. and estimated fraction of risk computalions were not

The RPD is periormed when both the main value and, ether, the duplicate, split, or regulator solt values are greater than 5 times the targat detection limit (TOL). These RPD calcuations use the following lormula $R P D=[M-S \mid(M+S ; / 2)]^{* 100}$

where. $M=$ Man Sample Value

$S=$ Split (or dudicate) Sample Value

For QNQC split and duplicate RPD calculations, a value below $+1-30 \%$ indicates the data compare favorably. For regulatory splits. a threshold of $+1-35 \%$ is used (EPA $540 / R$. 94/013) If the RPO is grealer than $\% .30 \%$ (or $+1-35 \%$ for regulatory spit dara). further investigation regarding the usability of the dala is performed. Additional discussion as necessary is provided in the data qualty assessrient section of the applicable CVP

If regulator split comparison is required. an additusnal parameler is evaluated. A control limit of +1.2 limes ine TDL shall be used if either the main or regulator spit value is less than 5 times the TOL and above detection in the case: where only one resull is above the five limes the TDL and the other is below, the $+1-2$ times the TDL criteria applies. Therefore the rollowing calculation is performed cuning these two cases involving regulator split dallin

If the cifference is greater than +1- 2 tmes the TDL, tren turther investigation regarding the useability of the data is performed and presented in the applicable CVP data qualty assessment. 


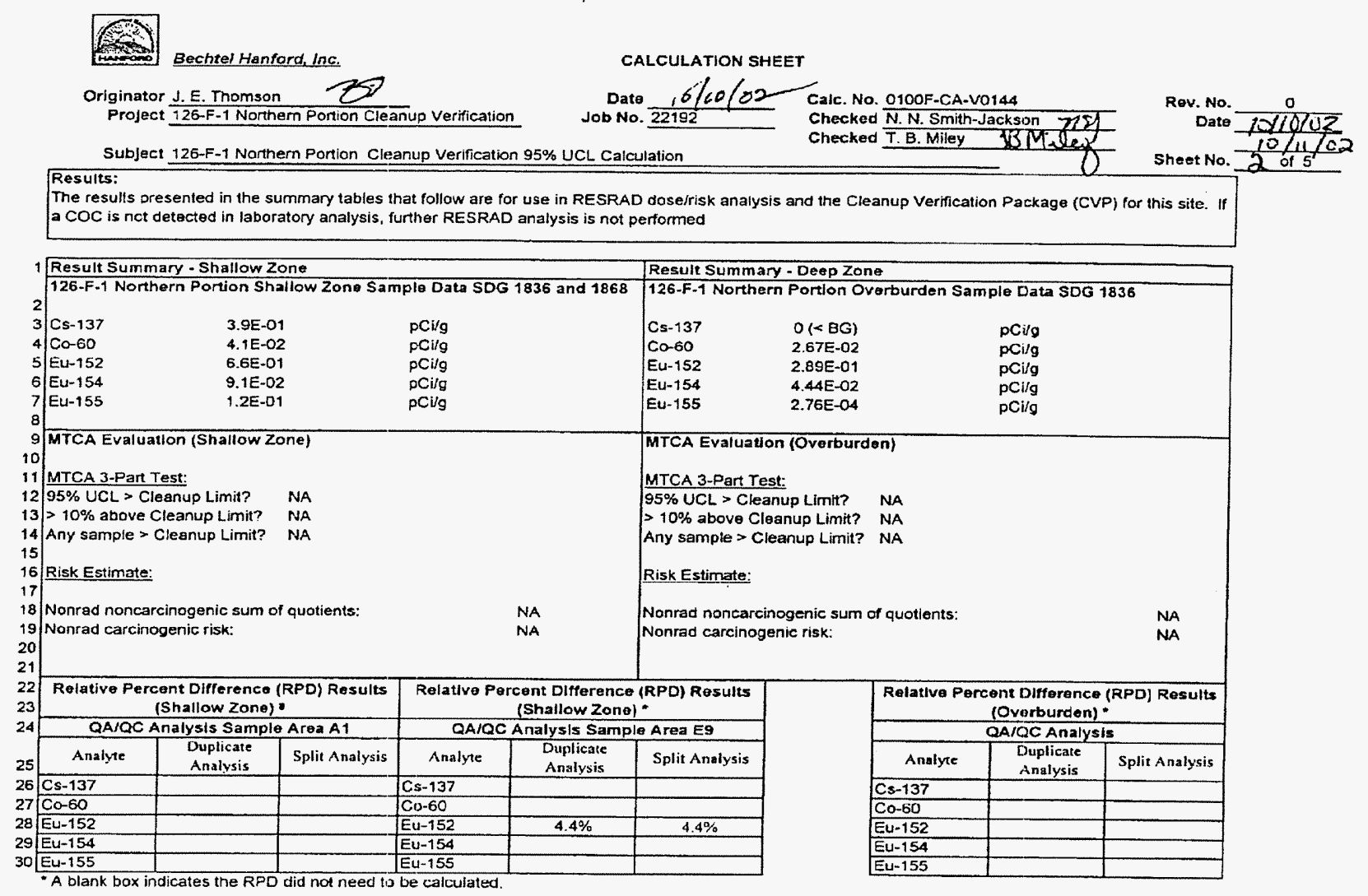




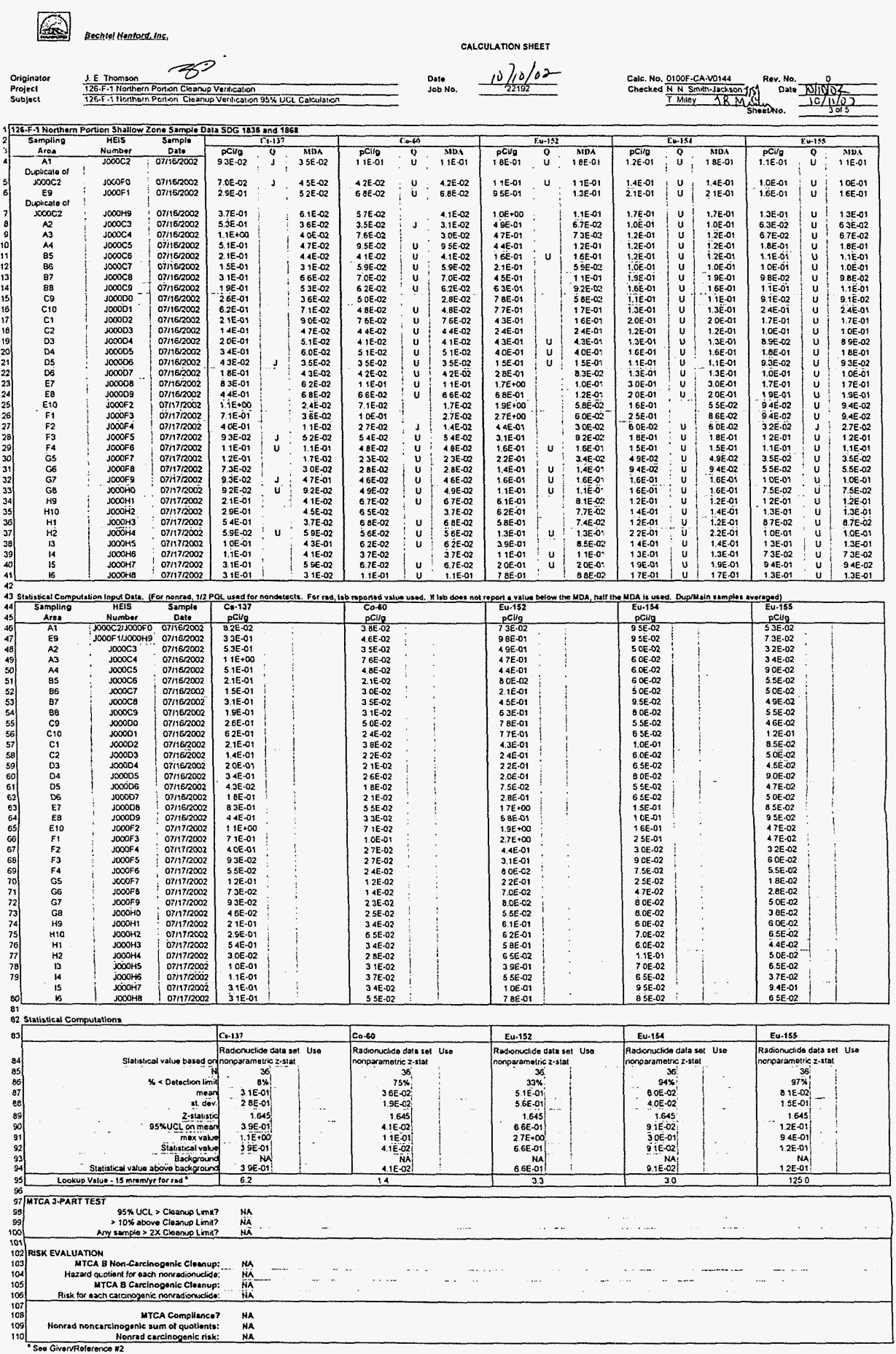




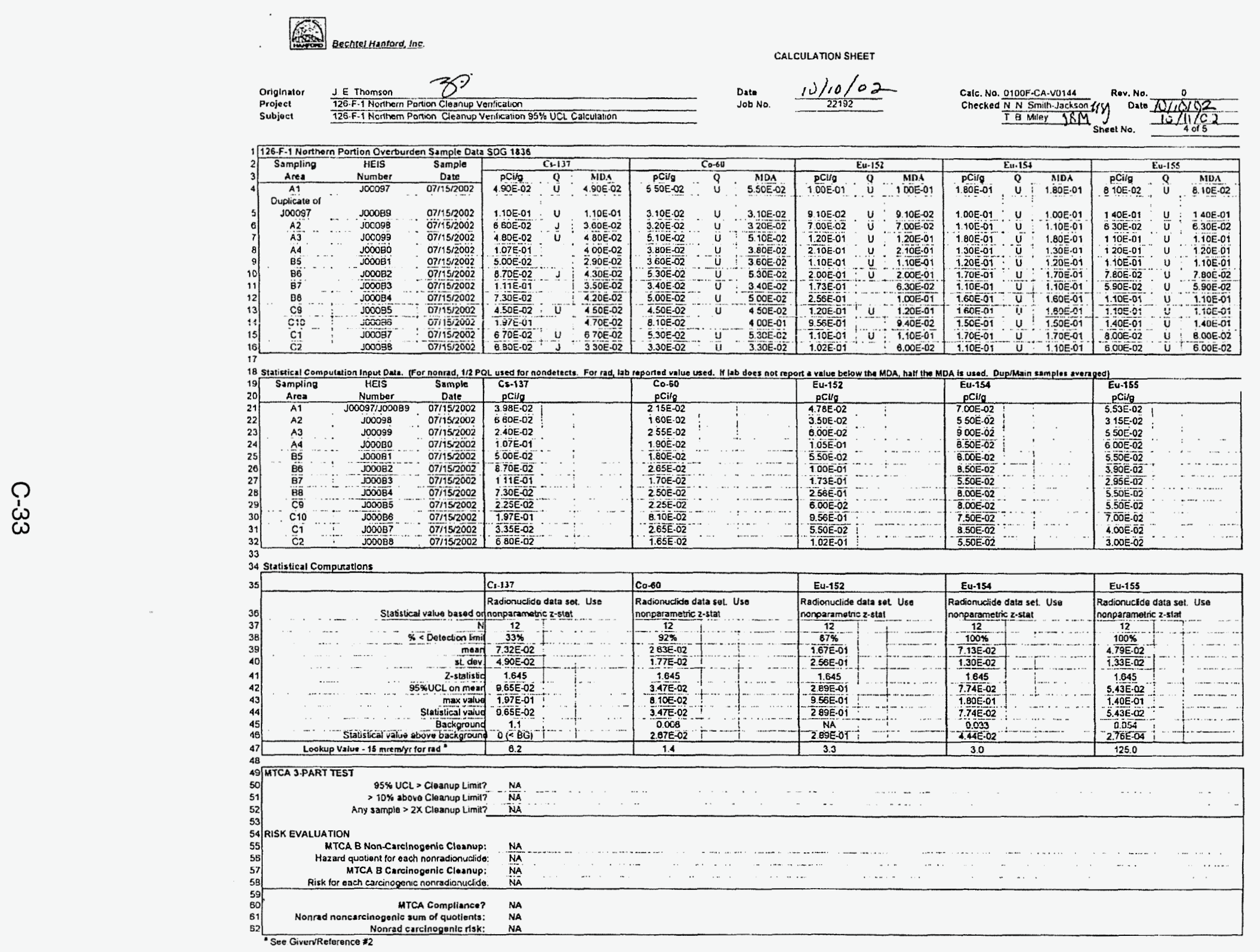


Originator J.E. Thomson 20

Projact 126.F-1 Norhem Portion Cleanup Venilication

128 -F-1 Northem Portion Cleanup Vorificailion $95 \%$ UCL Calculation

Spliciduplicate Andysis

Splicouplicere Analyzis:

\section{CALCULATON SHEET}

$\operatorname{Date}_{\text {Job No. }} \frac{10 / 10 / 02}{22192}$
Calc. No.: O100F-CA.V0144

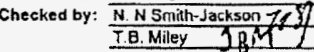

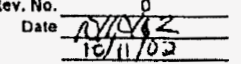

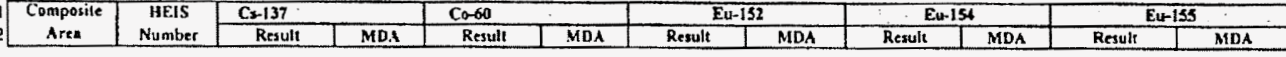

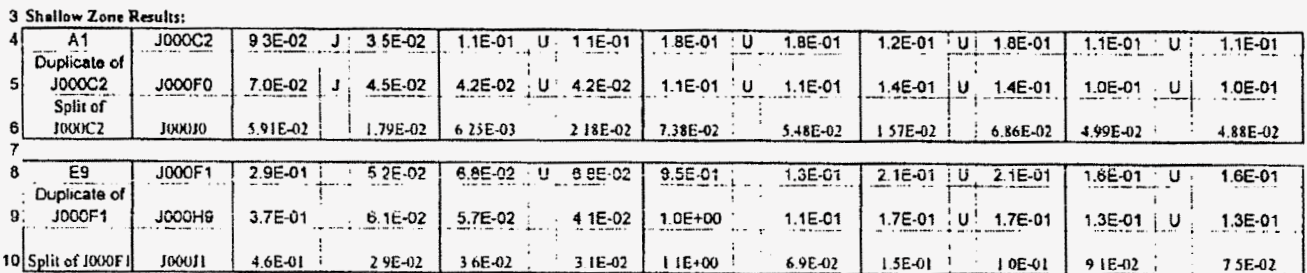

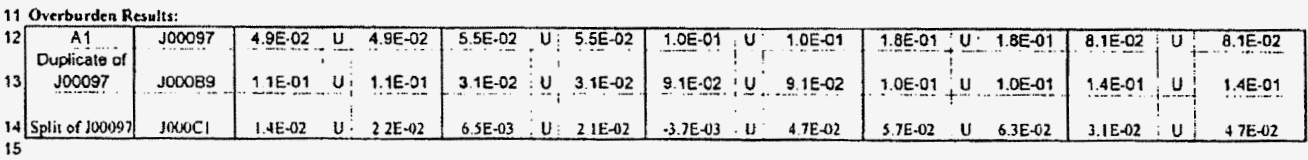

\begin{tabular}{|c|c|c|c|c|c|c|}
\hline$\pi$ & & 01 & 0.05 & 0.1 & 0.1 & 0.1 \\
\hline \multirow{3}{*}{$\begin{array}{l}\text { Duplicart: } \\
\text { Analysis }\end{array}$} & $\begin{array}{l}\text { Boths? } \\
\text { MDAs? }\end{array}$ & $Y_{E}(\operatorname{con} t i n u \in)$ & & & & No-Silop (acappabie) \\
\hline & $\begin{array}{c}\text { Both } \\
25 \times \mathrm{TLL} \text { : }\end{array}$ & No-Stop (acueptable) & No-Stop (accaptable) & No-Stop (2copplable) & No-Stop $(\operatorname{sicsptablve)}$ & No-Sicog $(\operatorname{ccccotable})$ \\
\hline & RPD & & & & & \\
\hline \multirow{2}{*}{ Split Analy sis } & $\begin{array}{l}\text { MDA? } \\
\text { MDA: }\end{array}$ & Yes (continue) & No-Stop $(\{x<\mathrm{cp} u b l i)$ & No-Stop (accoptublc) & No.Stop (xactplables) & $N_{0}-\operatorname{Siop}(\operatorname{accepptable)})$ \\
\hline & $\begin{array}{l}\text { Both } \\
\text { >S<CTD1.? }\end{array}$ & No-Stop (2xceptable) & No-Stop (accappably) & Na-Siop (acospabic) & No-Stop (ascopplable) & No-Slop (accoptable) \\
\hline \multicolumn{7}{|c|}{ Analyris: } \\
\hline \multirow{3}{*}{$\begin{array}{l}\text { Duplicalc } \\
\text { Analysis }\end{array}$} & $\begin{array}{l}\text { Bolt's } \\
\text { MDA? }\end{array}$ & Yes (continue) & 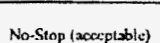 & Yrs (continus) & No-S Sug tocceptablet & 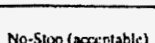 \\
\hline & $\begin{array}{c}\text { Both } \\
\rightarrow \text { SNTOL? }\end{array}$ & No-Stop (accupplable) & & Yess (Calc RPD) & & \\
\hline & $\mathrm{RPO}$ & & & $4.4 \%$ & & \\
\hline \multirow{3}{*}{ Split Analysis } & $\begin{array}{l}\text { Boths } \\
\text { MDA? }\end{array}$ & Yes (continue) & No-Stop \{acappiable & Yes icontinut) & No-Stop (acceptable) & No-Sloo (2000.ptable $)$ \\
\hline & $\begin{array}{l}\text { Both } \\
>>_{\mathrm{S}} \mathrm{TDL} \text { ? }\end{array}$ & No - Stop (sectpable) & & Yes (cuic RPD) & & \\
\hline & RPD & & & $4.4 \%$ & & \\
\hline \multicolumn{7}{|c|}{ Overburden Analysis: } \\
\hline \multirow{3}{*}{$\begin{array}{l}\text { Duplicate } \\
\text { Analysis }\end{array}$} & $\begin{array}{l}\text { Both? } \\
\text { MIPA? }\end{array}$ & No-Stop (asceptable) & No. Stop (accoptables) & No-Swp (scceppable) & No-Stop (acceptable) & No-Stop (xecuptable) \\
\hline & $\begin{array}{c}\text { Both } \\
2 \times 5 \mathrm{TDL} \text { ? }\end{array}$ & & & & & \\
\hline & RPD & & & & & \\
\hline \multirow{3}{*}{ Split Analyssis } & Bout- & No-S S & 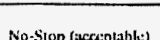 & Nor-Stap (acceptobles) & $N-5$ sis & $N-S$ \\
\hline & Buth & & & & & T \\
\hline & $\frac{P \text { SNTDL? }}{R P D}$ & & & & & \\
\hline
\end{tabular}




\section{CALCULATION COVER SHEET}

Project Title:

Area

Discipline

Subject

Computer Program
126-F-1 (Southern Half) Site Closeout $100-\mathrm{F}$ Environmental \begin{tabular}{ll}
$\frac{126-F-1 \text { (Southern Half) Cleanup Verification } 95 \% \text { UCL Calculation }}{\text { Excel }}$ & Program No. Excel 97 \\
\hline
\end{tabular}
Job No. 22192

Committed Calculation

$\mathrm{x}$

Preliminary

Superseded

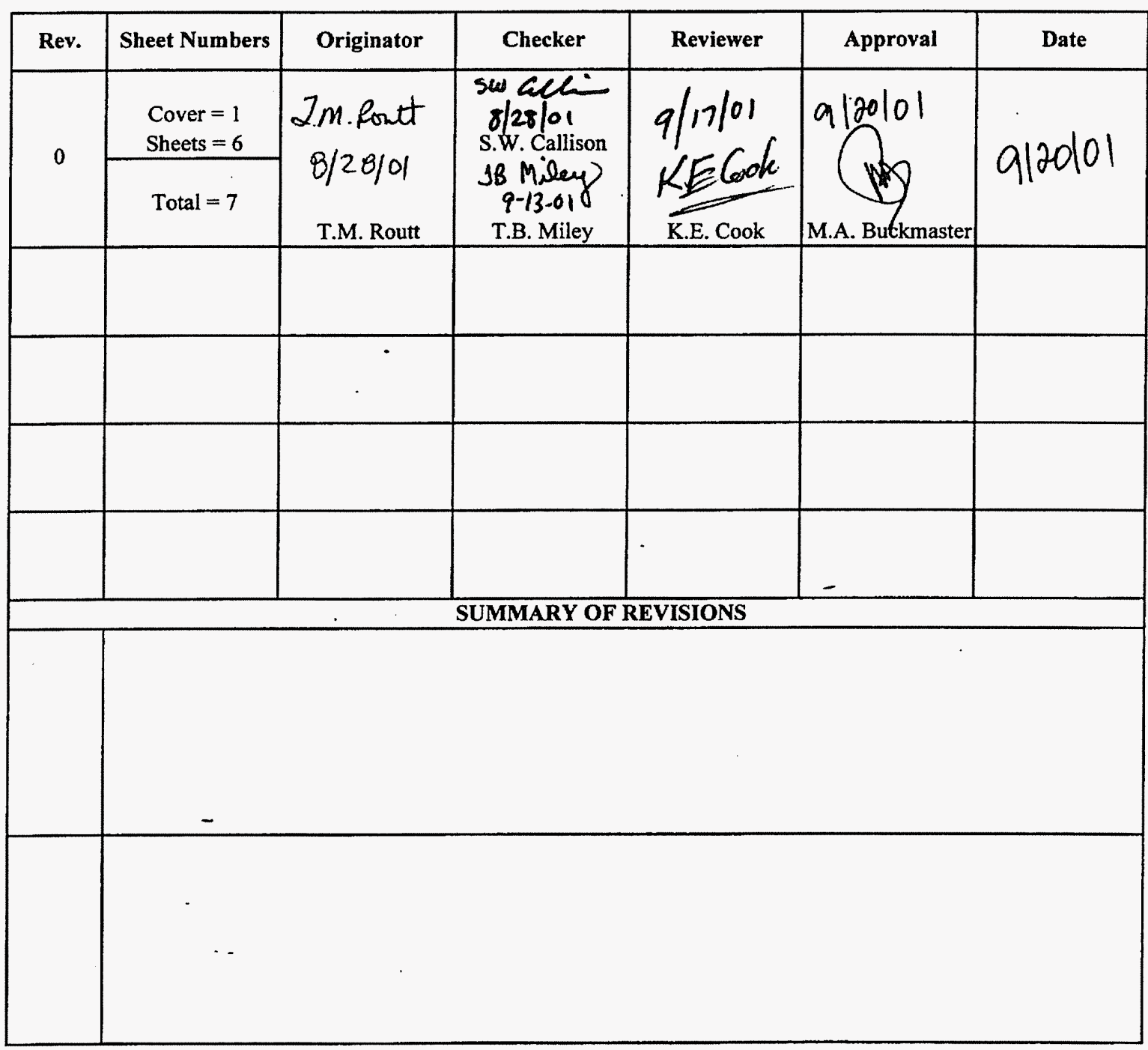

* Obtain calc no. from DIS

DE01-437.03 


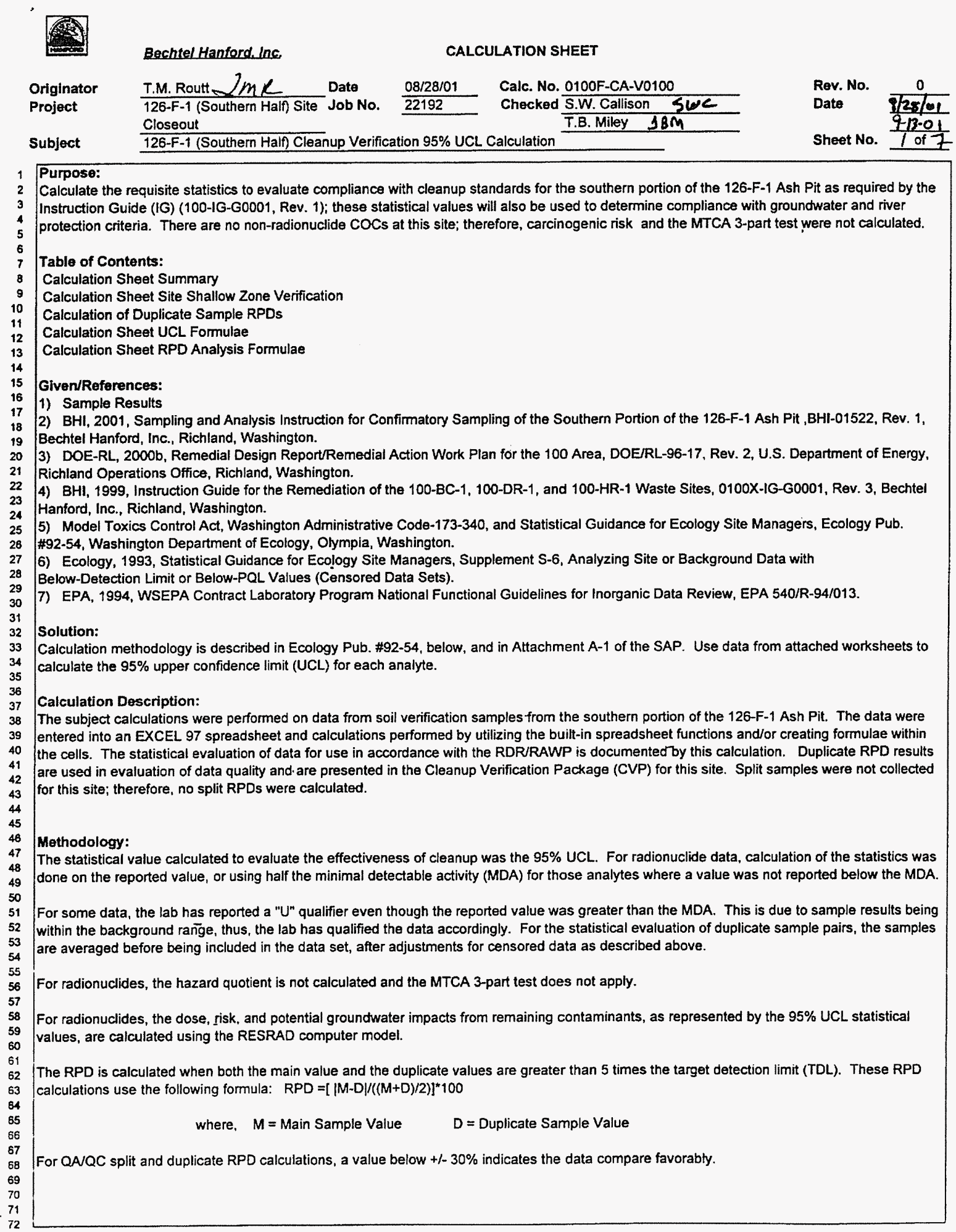




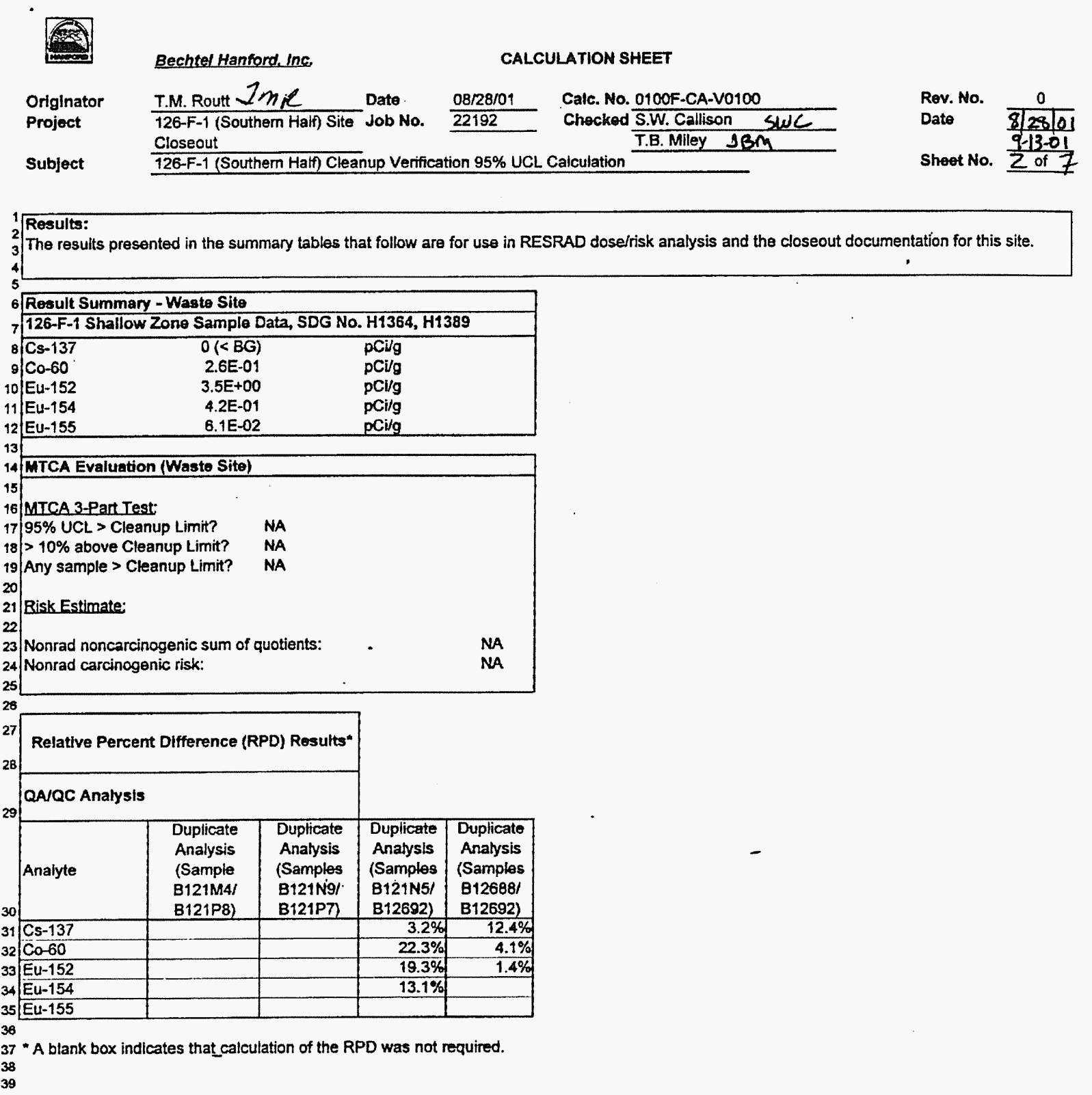




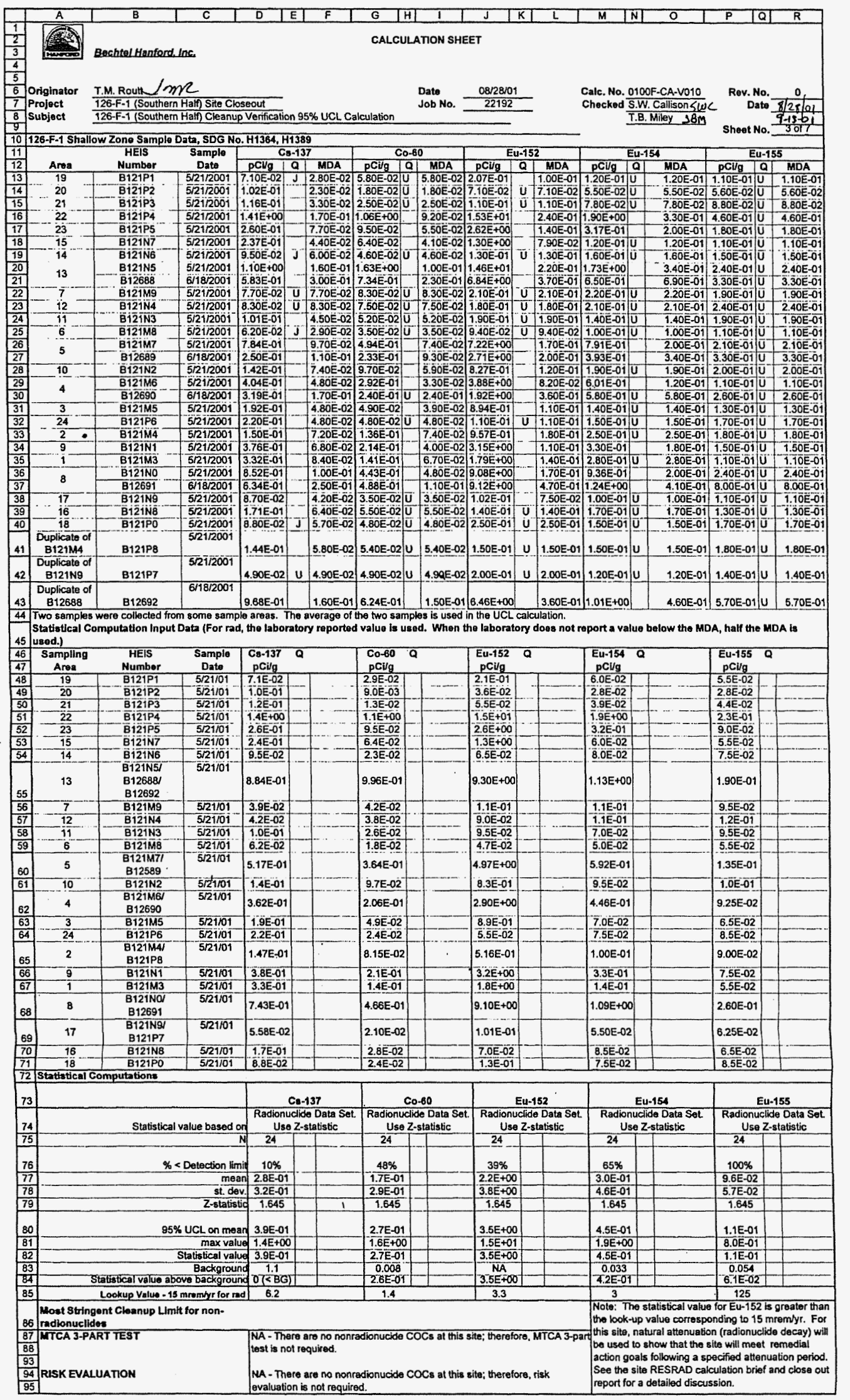




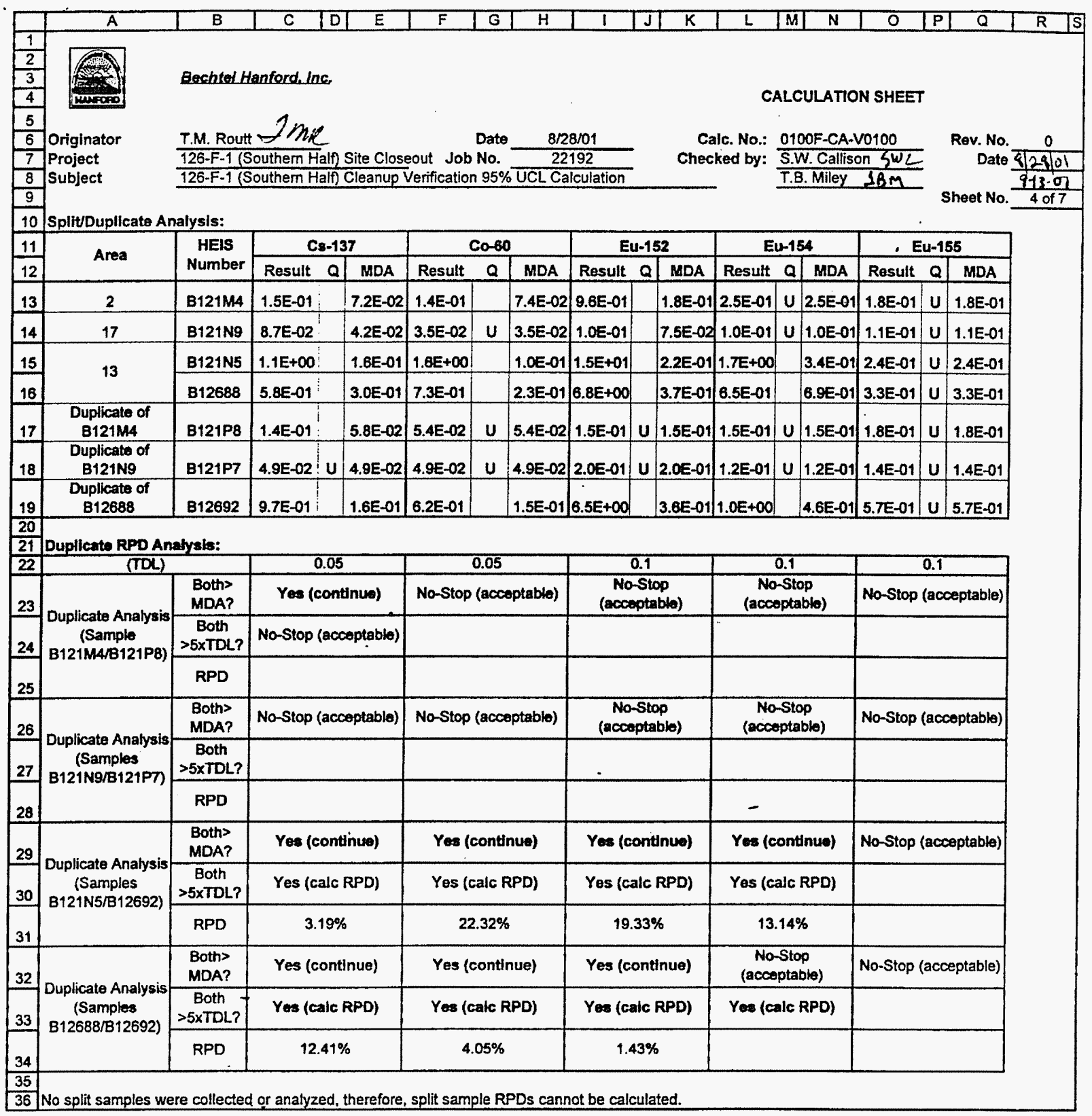




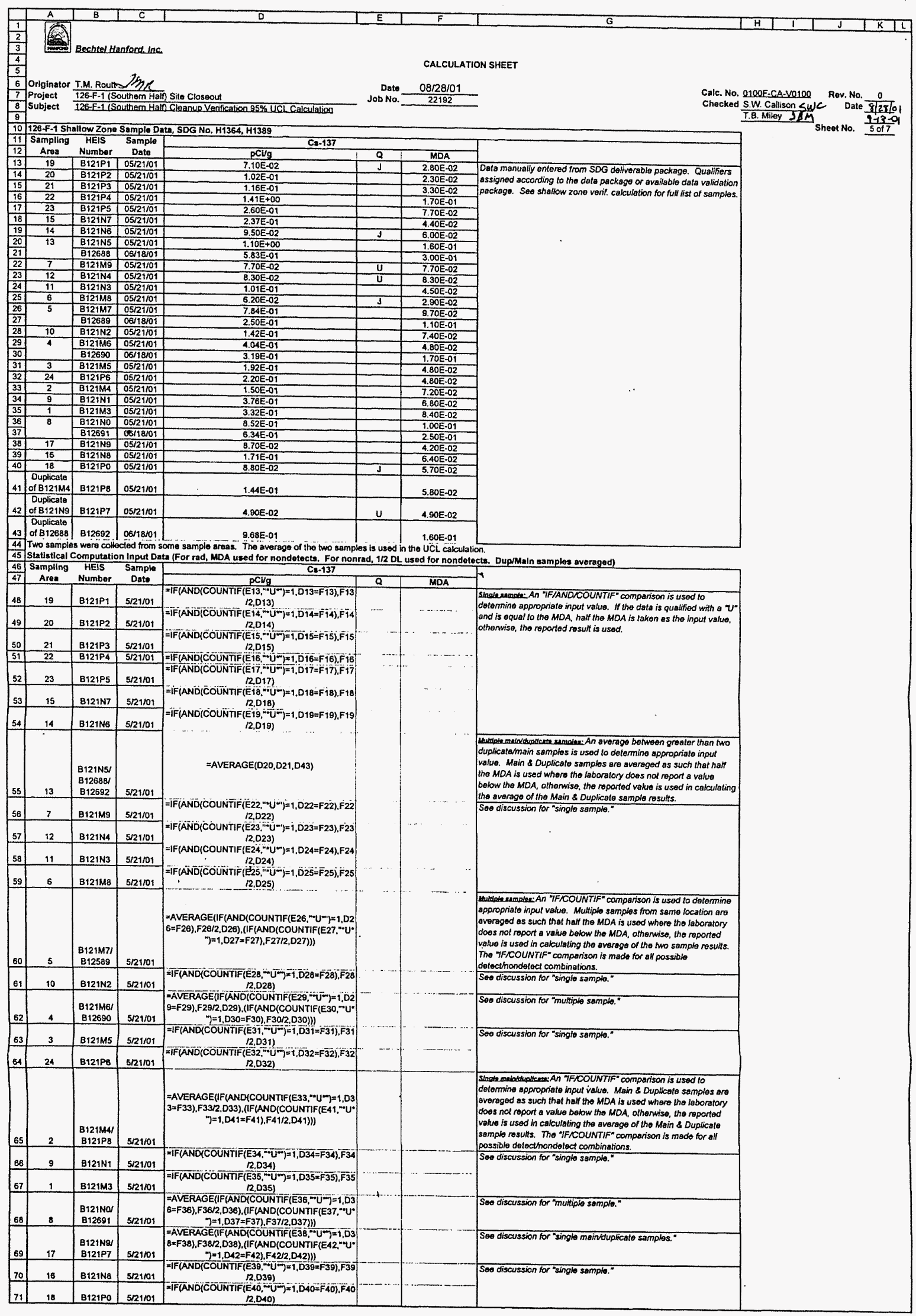




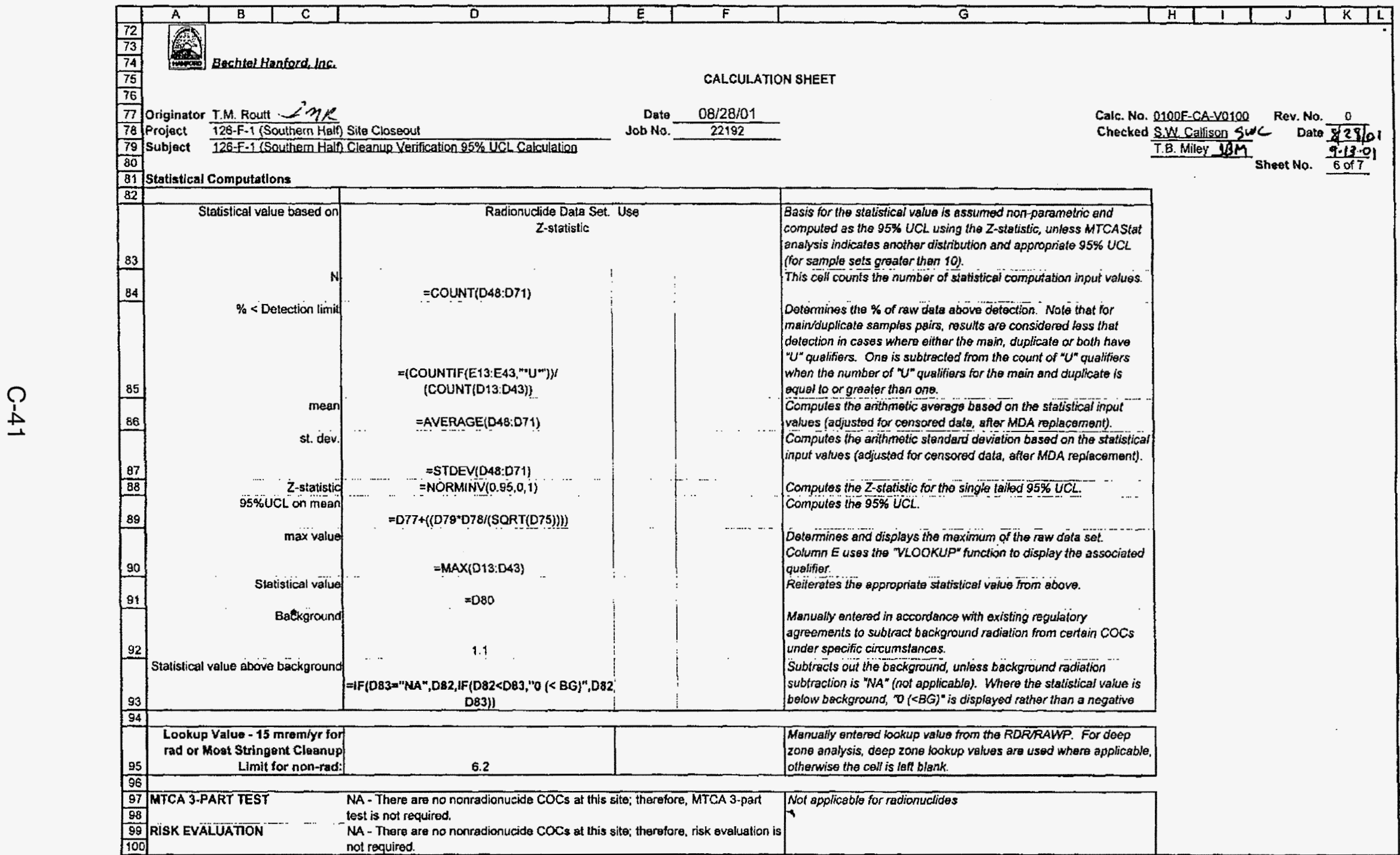




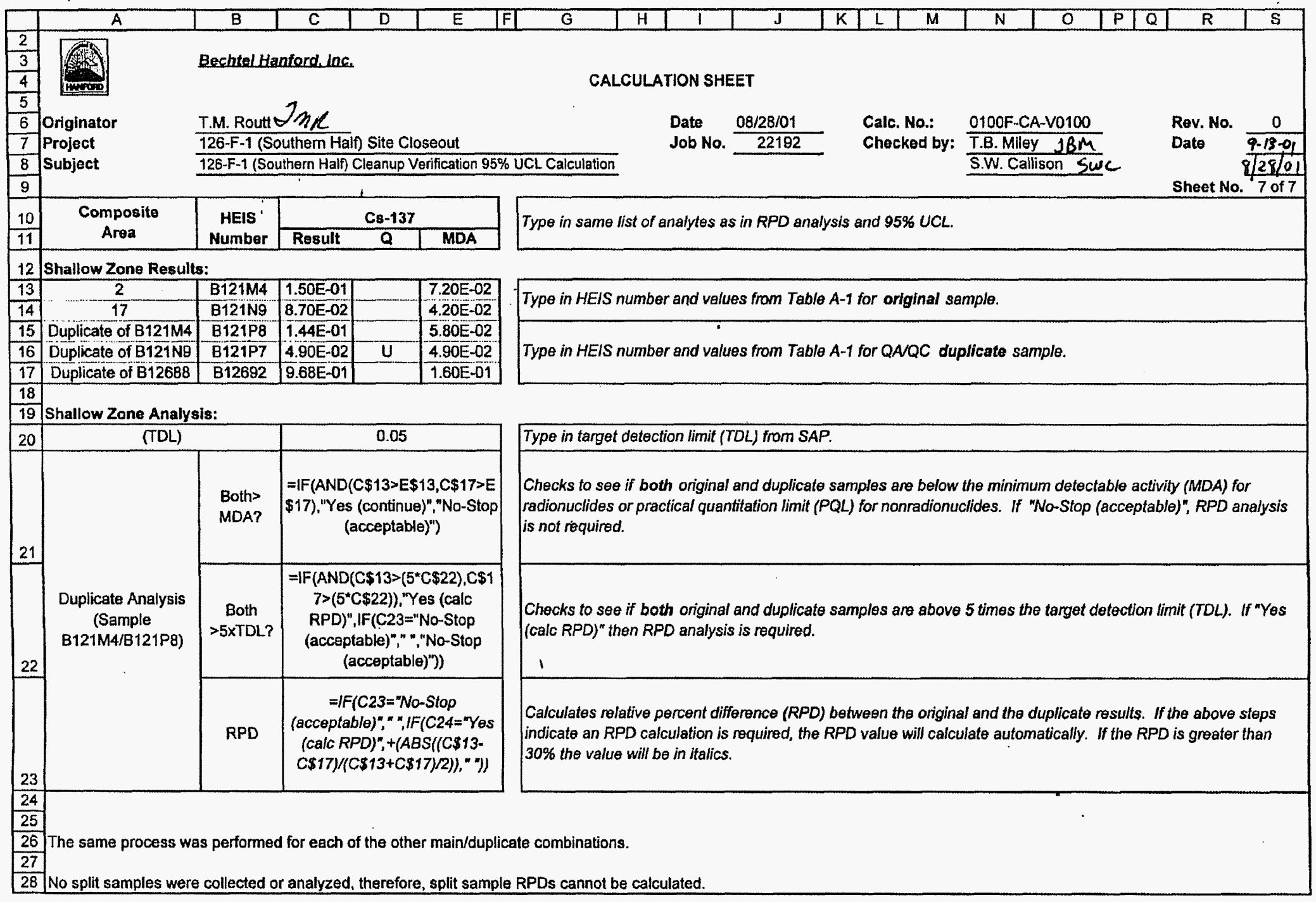




\section{CALCULATION COVER SHEET}

Project Title 126-F-1 Ash Pit Cleanup Verification Job No. 22192

\section{Area}

Discipline

Subject

Computer Program RESRAD

100-F Area

Environmental

*Calc. No.

0100F-CA-V0146

126-F-1 Ash Pit (Northern Portion) RESRAD Calculation

Program No.

Version 6.21

Committed Calculation $₫ \quad$ Preliminary $\square \quad$ Superseded

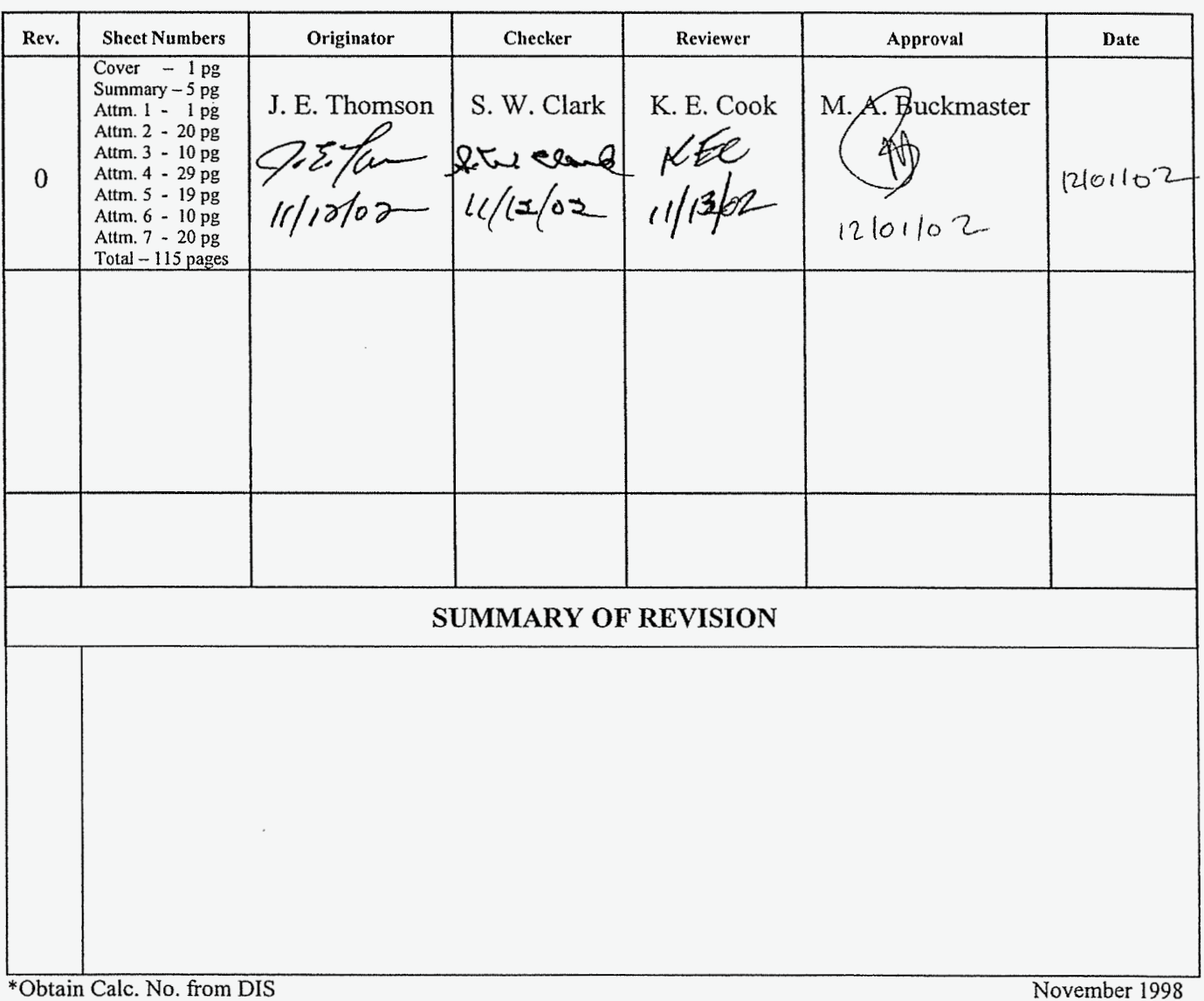

DE01-437.03 


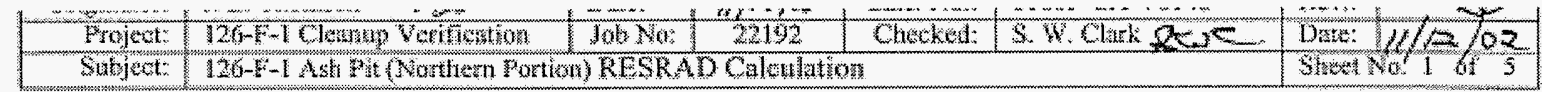

\section{PURPOSE:}

2

Calculate the soil and groundwater concentrations, dose, and risk contributions from remaining radionuclide contaminants in shallow zone and overburden soil in the northern portion of the 126-F-1 Ash Pit wemediation site over a period of 1,000 years.

\section{GIVEN/REFERENCES:}

1. Cleanup verification data from $126-F-1$ Norther Portion Cleanup Verification $95 \%$ UCL Calculation, Calculation No. 0100F-CA-V0144, Rev, 0, Beehtel Hanford, Ine, Richland, Washington.

2. Renedial Design Report/Remedial Acton Work Plan for the 100 Area (RDR/RAWP), DOE/RL-96-17, Rev. 3, U.S. Department of Energy, Richland Operations Office, Richland, Washington.

3. Radioactive contaminants of concern from the 100 Area Remediat Action Sampling and Analysis Plan (100 Area SAP), DOERL-96-22, Rev. 3, U.S. Department of Energy, Richland Operations Office, Richland, Washington. For the purpose of these RESRAD calculations, the radioactive contaminants of concern (COCs) are cobalt-60, cesium-137, etropium-152, etropium-154, and europium-155. There are no nonradiontuclide $\mathrm{COC}$.

4. RESidnal RADioactivity (RESRAD) computer code, version 6.21 , to calculate compliance with residual madionetivity guidelines, developed for the U.S. Department of Energy by the Environmental Assessment Division of Argone National Laboratory, Argonne, Illinois.

5. Sample design data from $126-F-1$ Ash Pit Shallow Zone Design Calculation No 0100FCA-V01 18, Rev. 0. Bechtel Hanford, Inc, Rhehland, Washington and $126-F-1 / \mathrm{sh}$ pif Overburden Design, Calculation No. 0100F-CA-Vo061, Rev, 0, Bechtel Hanford, Inc. Richland, Washington.

6. Ecology, EPA, and DOE, 1998, Hanford Federal Facility Agreement and Consent Orater, 2 wols, as anended, Washington State Department of Ecology. U.S. Environmental Protection Ageney, and U.S. Department of Energy, Olympia, Washington. 


\begin{tabular}{|c|c|c|c|c|c|c|c|}
\hline \multicolumn{8}{|c|}{ CALCULATONSHETI } \\
\hline Origistinkr: & 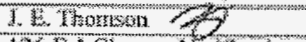 & 1,4012 & 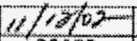 & Can No: & $61610 \times C A-V 116$ & Kex: & 8 \\
\hline Pragat: & 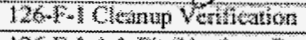 & Job Ne: & 22153 & Clecked: & SW. Clk $, \mathrm{C}, \mathrm{C}$ & Date: & $u / c 1$ \\
\hline Subjoet? & 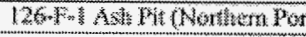 & 1) KRESK & 13 Cusculastio & & & Sket & \\
\hline
\end{tabular}

\section{SOLUTION:}

1) Separate RESRAD runs were performed for the relevant vadose zone soll horizons (shallow zone and overburden soil). Table 1 shows the elevations (NAVD88) and dimensions of each soil horizon. The shallow zone verification sumple data set is being used to conservatively represent the entire Vadose Zone below tho site. Conservative assumptions were made to simplify the process for estimating dose, excess lifetime cancer risk, and groundwater concentrations at the $126-\mathrm{F}-1$ site, woh that it was assumed that that the concentrations of contaminants detected in the shallow zone soils were consistent to the gronnewater table. The total-plan view area of the excavation was used to conservatively estimate the site area input parameter for each soil horizon.

A separate RESRAD run was performed using the verffication samples collected from the overburden soils. Because overburden soll was used to backfill the site excavaton, the contamituated zone thickness model assumes that the contamination is equat to the depth of the remedial site exeavation.

Attachment 1 shows the dmensions of each sol borkon and the contaninant pathways considered for dose, risk, and groundwater protection. RESRAD input parameters for each run arc shown in the "Summary" section of the RESRAD "Part I: Mixture Sums and Single Radionuclide Cutclines" printouts in the Attachments to this Caleulation Summary.

Table 1. Dimensions and Clevation (NAYD88) of 126-F-1 Soil Horizons

\begin{tabular}{|c|c|c|c|c|}
\hline RESRAD RUm & Vadose Zone Karizon & $\begin{array}{l}\text { Upper } \\
\text { Elevation (m) }\end{array}$ & $\begin{array}{c}\text { Lew } \\
\text { Elevation }(\mathrm{m})\end{array}$ & Thichness (m) \\
\hline 1- Raxinowselides & $\begin{array}{l}\text { Vadose Zone } \\
\text { (Shallow zane data sey) }\end{array}$ & 123.8 & 115.0 & 8.8 \\
\hline 2.- Katrontuclides & Owarturden & 123.8 & 119.8 & 4.0 \\
\hline
\end{tabular}

2) The year where the peak dose (or concentration) occurs from each individual radoutollo COC and layer is determined by a prelimnary nu. This year is then ineuded for all horizons for the final RESRAD runs. For the direct exposure pathway (i.e. soil ingestion. and inhatation and external radiation, the peak year occured at year zero (year 2002) for all radionuclide COCs. For the water patwwy (i.e. drinking water and food ingestion) the peak year was 7 for cobalt-60, and 43 for cesium-137. The $7-$ and $43-y e a r$ thine periods were added to the RESRAD runs. The year 2018 is included for evaluation in all horizons (the year 16 timemeriod as 2018 corresponds to the 30 -year site clearun schedule of the 
Bechtel Hanford, Inc.

CALCULATION SHEET

\begin{tabular}{|c|c|c|c|c|c|c|c|}
\hline \multicolumn{8}{|c|}{ CALCULATION SHEET } \\
\hline Originator: & 1. E. Thomson & Date: & $11 / 3102$ & Cate No.: & 010EF $* \mathrm{CA} * \mathrm{VMC6}$ & Rer.: & \\
\hline Moget: & $126-1 \%$ C Cleanup Vertication & IकW No & 22192 & Chiteked: & S. W. Clark J $\mathrm{X}$ & Date: & $11 / 12 / 02$ \\
\hline Subiect: & \multicolumn{5}{|c|}{ 126.F 1 Asth Pit (Worthern Portion) RESRAD Calcalation } & Sheet? & 0.3 of 5 \\
\hline
\end{tabular}

1

\section{METHODOLOGY:}

1) Runs of RESRAD version 6.21 were completed for the shallow zone and overburden soil using the radionuclide concentrations shown in Table 2. RESRAD numerical output reports for radionuclide dose, risk, and concentration for the shallow zone and overburden soil are presented in the Attachments to this Calculation Summary.

Table 2. Cleanup Verification Data Set

\begin{tabular}{|c|c|c|}
\hline \multirow{2}{*}{ Radionuclide COCs } & \multicolumn{2}{|c|}{ Radionuclide Activity, pCl/g } \\
\hline & Shallow Zone & Overburden \\
\hline $\mathrm{Cs}-137$ & $3.9 \mathrm{~L}-01$ & $0(<0 G)^{5}$ \\
\hline Co-60 & A.1E-02 & $2.7 \mathrm{E}-12$ \\
\hline $\mathrm{Eu}=152$ & $6,6 \mathrm{E}-01$ & $2,98-01$ \\
\hline $\mathrm{En}-154$ & $9.1 \mathrm{E} 402$ & $4,4 \mathrm{E} m(\mathrm{O}(\mathrm{W})$ \\
\hline $2 \mathrm{a}-155$ & $1.2 \mathrm{E} 4 \mathrm{I}$ & $2,660.64)^{3}$ \\
\hline
\end{tabular}

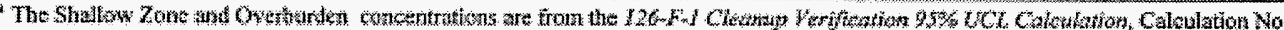

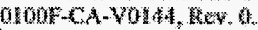

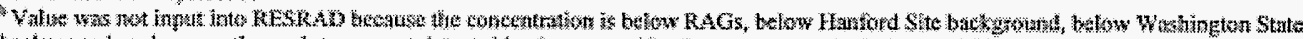

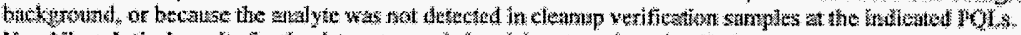

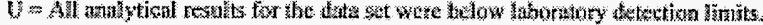

10

\section{RESULTS:}

\section{1) Radionuclide "All Pathways" Dose Rate}

The "all pathways" (maximum) dose rates are shown in Table 3. The vadose zone maximum total all pathways dose rate of $4.8 \mathrm{mrem} / \mathrm{yr}$ occurs at year zero (2002). The vadose zone all pathways dose rate for year 2018 (time slice 16) is $2.1 \mathrm{mrem} / \mathrm{yr}$. The maximum overburden dose rate of 1.6 mrem/yr occurs in year zero.

Table 3. All Pathways Dose Rate (mrem/yr)

\begin{tabular}{|c|c|c|c|c|c|c|c|c|c|c|}
\hline \multirow{2}{*}{$\begin{array}{l}\text { RESRAD } \\
\operatorname{Ran}\end{array}$} & \multirow{2}{*}{$\begin{array}{l}\text { Vadose Zne } \\
\text { Horiogrs }\end{array}$} & \multicolumn{9}{|c|}{ All Pathways" Dose Contributions in mrem/yr at Each Time Slice (yr) } \\
\hline & & 0 & 1 & 3 & 7 & 16 & 43 & 100 & 300 & 1600 \\
\hline 1 & Simallow Zane & $4.3 x+4 x$ & $4.5 \mathrm{E}+00$ & $4.11+6$ & $3.32+60$ & $21 \mathrm{~m}+60$ & $70 \mathrm{E}-101$ & 1. $2 E-01$ & $9.9 \mathrm{E}-0.6$ & $9.5 \mathrm{~d} \times 11$ \\
\hline $\begin{array}{r}\text { Total } \\
\text { pathways }\end{array}$ & 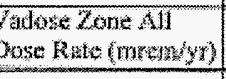 & 4.8 Fon & $4.5 F+m$ & $4,14+6$ & $3.32+00$ & $2.1 E+00$ & $7, w_{1}-1$ & $1,2 \mathrm{E}-0$. & $995-04$ & $9.5 E_{-11}$ \\
\hline 2 & Oveburaten & $16 \mathrm{E}+00$ & $1,5 \mathrm{~s}, \mathrm{and}$ & 1.3\% 300 & $1.0 E++\infty$ & $5 \% \mathrm{E} A 1$ & $1,4 \mathrm{E}$ & $70 \mathrm{H} \cdot 63$ & 2.1E 07 & $5.0 \mathrm{E}-16$ \\
\hline
\end{tabular}


Theres: Bechtel Hanford, Inc.

CALCULATION SHERT

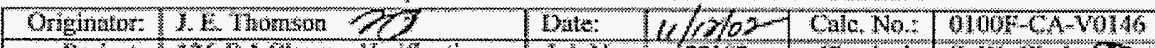

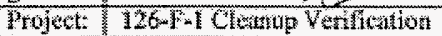

$10 \mathrm{Na} / 221 \% 2$ Checked

Subject: / 26-1\%1 Ash pit (Nerthera Portien) RESRAD Calculation

\section{2) Radionuchide Excess Cancer Risk}

The radionuclide excess lifetime cancer risk results are shown in Table 4. The vadose zone maximum total excess lifetime cancer risk $\left(5.8 \times 10^{-5}\right)$ occurs at year zero (2002). The vadose zone excess lifetime cancer risk for year 2018 (time slice 16 ) is $2.8 \times 10^{-5}$. The maximum risk from owerburden soll $\left(1.7 \times 10^{5}\right)$ ocetrs in year zero.

Table 4. Radionuclide Excess Lifetime Cancer Risk

\begin{tabular}{|c|c|c|c|c|c|c|c|c|c|c|}
\hline \multirow{2}{*}{$\begin{array}{c}\text { RESRAD } \\
\text { Rum }\end{array}$} & \multirow{2}{*}{$\begin{array}{l}\text { Vetore Zouse } \\
\text { Horizons }\end{array}$} & \multicolumn{9}{|c|}{ Excess Cancer Risk at Each Time Slfoe (yr) } \\
\hline & & 0 & I & 3 & 7 & 16 & 43 & 100 & 300 & 1060 \\
\hline 1 & Shallow Zone & $\$, 82-05$ & $5,5 \mathrm{H}-0 \mathrm{~s}$ & $50 \mathrm{OE}$ & 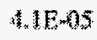 & $2.8 E n 05$ & $9.9 \mathrm{E}-06$ & $1.8 \mathrm{E}, 06$ & $1,6 \mathrm{E}=08$ & $1.5 E^{2}=5$ \\
\hline \multicolumn{2}{|c|}{ 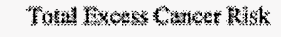 } & $5,8=05$ & $5.3 \mathrm{~L} * 05$ & $50 \mathrm{E} 0 \mathrm{~S}$ & 4. $1 \mathrm{E}-25$ & $2.8 \mathrm{E}-05$ & $9.9 \mathrm{E}-46$ & 1.8E-66 & 1.6208 & $1.5 E-15$ \\
\hline 2 & Overburden & $1.75-05$ & $1,6 \mathrm{E}-05$ & $1,4 \mathrm{E}-05$ & $1.1 \mathrm{E}-05$ & $6.8 \mathrm{EE}+06$ & $16 \mathrm{E} \cdot 05$ & $8.3 \mathrm{E}+68$ & $2.5 \sin 12$ & $3.4 \mathrm{E} \mu \mathrm{z} 1$ \\
\hline
\end{tabular}

8

9

10

11

12

13

14

15

16

17

18

Table 5. RESRAD Predicted Groundwater (Well Water/Drinking Water) Concentrations

\begin{tabular}{|c|c|c|c|c|c|c|c|c|c|c|c|}
\hline \multirow{2}{*}{$\mathrm{COC}$} & \multirow{2}{*}{$\begin{array}{c}\text { Vadose Zone } \\
\text { Morizon }\end{array}$} & \multicolumn{9}{|c|}{ Groundwater Concentrations in pCWl at Each Thme slice (yr) } & \multirow{2}{*}{ 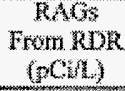 } \\
\hline & & 0 & 1 & 3 & 7 & 16 & 43 & 100 & 300 & 1000 & \\
\hline \multirow{3}{*}{ Co 60} & Skallow Zora & 0 & $9,27-05$ & $2.1 \mathrm{I} / \mathrm{H}$ & 2,94 & $2,11,44$ & 1.61 .05 & $20 \mathrm{~K} 0 \mathrm{~S}$ & $23 \mathrm{e}-19$ & 0 & \multirow{3}{*}{ lop } \\
\hline & Wadine Zane futal & 0 & $92.10 \times 15$ & $2.11+4$ & 294,64 & $2,1 \mathrm{E}, 0$ & $1,6 \mathrm{E}, 05$ & $20 \mathrm{Ne} 0 \mathrm{~s}$ & $23 E-19$ & 0 & \\
\hline & Oyerbutusten & $B$ & $\frac{1}{4}$ & 0 & (1) & 0 & 0 & 0 & 0 & 0 & \\
\hline \multirow{2}{*}{$\mathrm{Cs}-137$} & Shallow 7 wne & 1 & 9.7804 & $2.86-63$ & $608-63$ & $1.12-02$ & $1.6 \%, 012$ & $9.4 \mathrm{E}-0$ & 2,9104 & $9 \mathrm{~F}^{\infty}$ & \multirow{2}{*}{60} \\
\hline & Yadose Zorie Total & 0 & 97 & 2.12.2.13 & 600503 & $1,1 \mathrm{E}_{*} \mathrm{t} 2$ & $1.61 \times 12$ & $991 \%$ & $29 \mathrm{k} \times 04$ & 8,9111 & \\
\hline \multirow{3}{*}{$2 u-152$} & Shillow Zone & 0 & 0 & 0 & 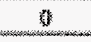 & 0 & 0 & 0 & 0 & 0 & \multirow{3}{*}{200} \\
\hline & Vaulose Znue Total & 0 & 9 & $y$ & D & $p$ & 3 & 0 & 0 & 0 & \\
\hline & Overounten & i & 0 & 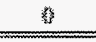 & 0 & 0 & 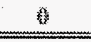 & 0 & 6 & a & \\
\hline \multirow{2}{*}{$\mathrm{Ka}-154$} & Siratlow Zune & $a$ & 0 & 6 & 0 & 0 & 6 & 0 & 0 & 9 & \multirow{2}{*}{60} \\
\hline & Youose Zone fotal & 0 & 0 & 0 & 0 & $n$ & 0 & 0 & 0 & 0 & \\
\hline \multirow{2}{*}{ Eu-15s } & Strilow Zone & 0 & 0 & 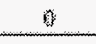 & 0 & 0 & 4 & 0 & 0 & 0 & \multirow{2}{*}{600} \\
\hline & Vadose Zone Tatal & 0 & 0 & 0 & 0 & 0 & 0 & 3 & 0 & b & \\
\hline
\end{tabular}

\section{3) Radionuclide Gronndwater Protection}

The radionuclide concentrations in groundwater calculated by the RESRAD model are summarized in Table 7. The organ specific dose via the groundwater (and river) pathway is calculated in a separate calculation brief. Only concentrations are presented here. Cobalt- 60 and cesium 137 were predicted to reach gromdwater in the 1,000 years of the RESRAD model run for the vadose zone. These radionuclide contaminants reach groundwater at concentrations significantly below the RAGs. Radionuclide contaminants of concern in overburden soil are not predicted to reach groundwater.

19 


\begin{tabular}{|c|c|c|c|c|c|c|c|}
\hline Orimator: & 1. 2 . Thomenom 27 & Drta: & $1,1<21,2$ & Colc, Ro: & $010 \mathrm{FF}=\mathrm{CA} \times 0146$ & $\mathrm{Rew}:$ & 1.0 \\
\hline Frojert: & $126-1+1$ Cheanup Verificetion & $\mathrm{Inb} \mathrm{No}^{x}$ & 22192 & Cheoked: & S. W. Chik hw & Date: & \\
\hline
\end{tabular}

\section{CONCLUSIONS:}

- The maximum total all pathways dose rate of 4.8 mrem/yr occurs at year zero (2002). The maximum overburden dose rate of $1.6 \mathrm{mrem} / \mathrm{yr}$ occurs in year zero. The all pathways dose rate for year 2018 (time slice 16 ) is $2.1 \mathrm{mrem} / \mathrm{yr}$.

- The dominant pathway for the dose rate is direct extemal exposure.

- The primary radionuclide contributing to the direct exposure pathway dose rate is europium- 152.

- The maximum total excess lifetime cancer risk $\left(5.8 \times 10^{-5}\right)$ occurs at year zero (2002). The maximum risk from overburden soil $\left(1.7 \times 10^{-5}\right)$ occurs in year zero. The excess lifetime cancer risk for year 2018 (time slice 16 ) is $2.8 \times 10^{-5}$.

- Among the radionuclide contaminants of concern in the vadose zone, cobalt-60, and cesium-137 are predicted to reach groundwater in the 1,000 years of the RESRAD model run. These radionuclides are predicted to reach groundwater at concentrations significantly below the RAGs. Radionuclide contaminunts of concern in overburden soll are not predicted to reach groundwater.

- None of the site COCs are projected to exceed remedial action goals (RAGs).

\section{ATTACHMENTS:}

1. Graphic showing 126-F-1 Cleanup Verification Model (1 page)

2. RESRAD Output: 126-F-1 Shallow Zone Rad (Run 1), Mixture Sums and Single Radionuclide Guidelines (20 pages)

3. RESRAD Output: 126-F - I Shallow Zone Rad (Run 1), Concentration of Radionuclides (10 pages)

4. RESRAD Output: 126-F-1 Shallow Zone Rad (Run 1), Intake Quantities and Health Risk Factors (29 pages)

5. RESRAD Output: 126-F-1 Overburden Rad (Run 2), Mixture Sums and Single Radionuclide Guidelines (19 pages)

6. RESRAD Output: 126-F-1 Overburden Rad (Run 2), Concentration of Radionuclides (10 pages)

7. RESRAD Output: $126-F_{-1}$ Overburden Rad (Run 2), Intake Quantities and Health Risk Factors (20 pages) 
126-F-1 Northern Portion Cleanup Verification Model

Vadose Zone contribution to
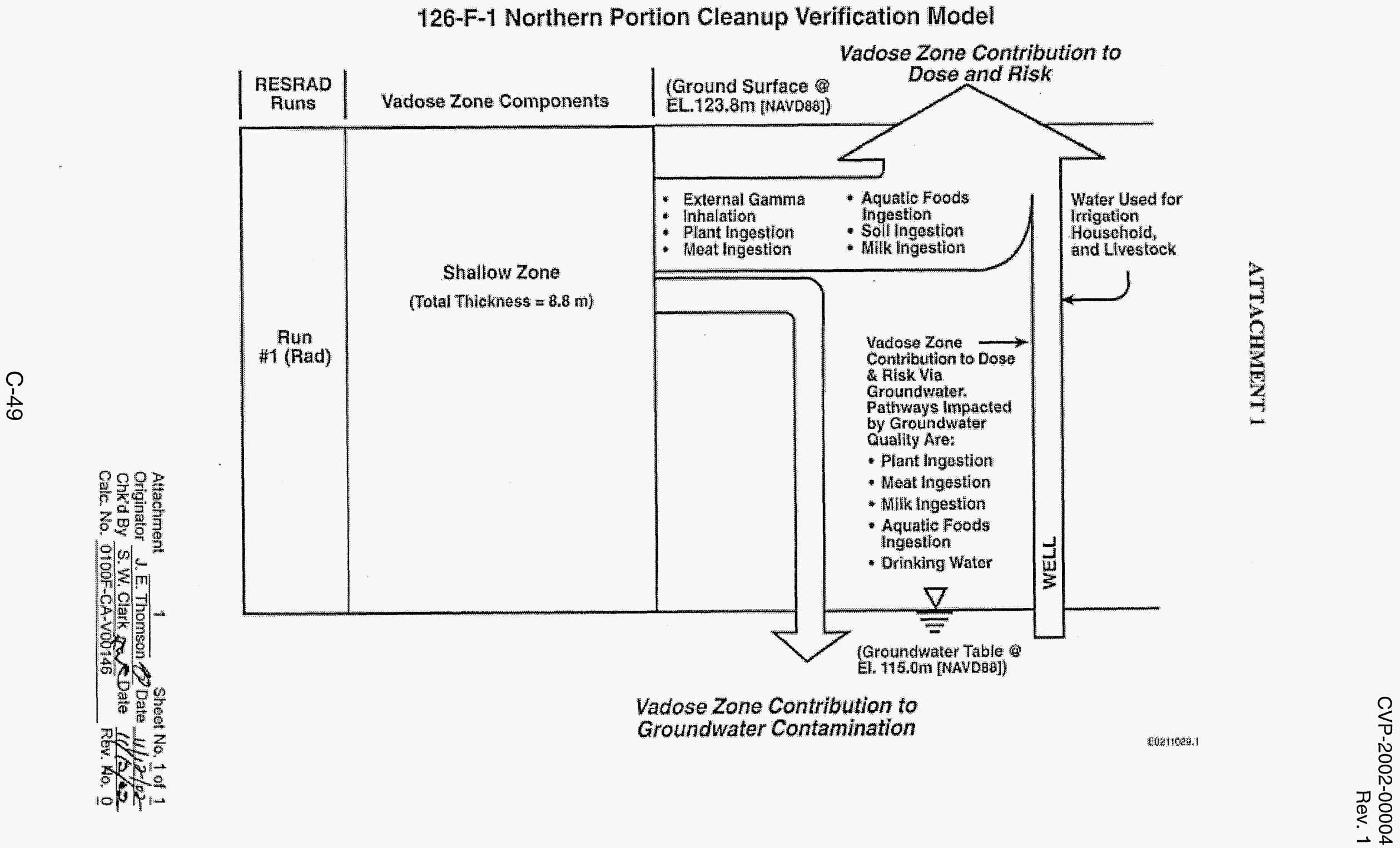


\section{ATTACHMENT 2}

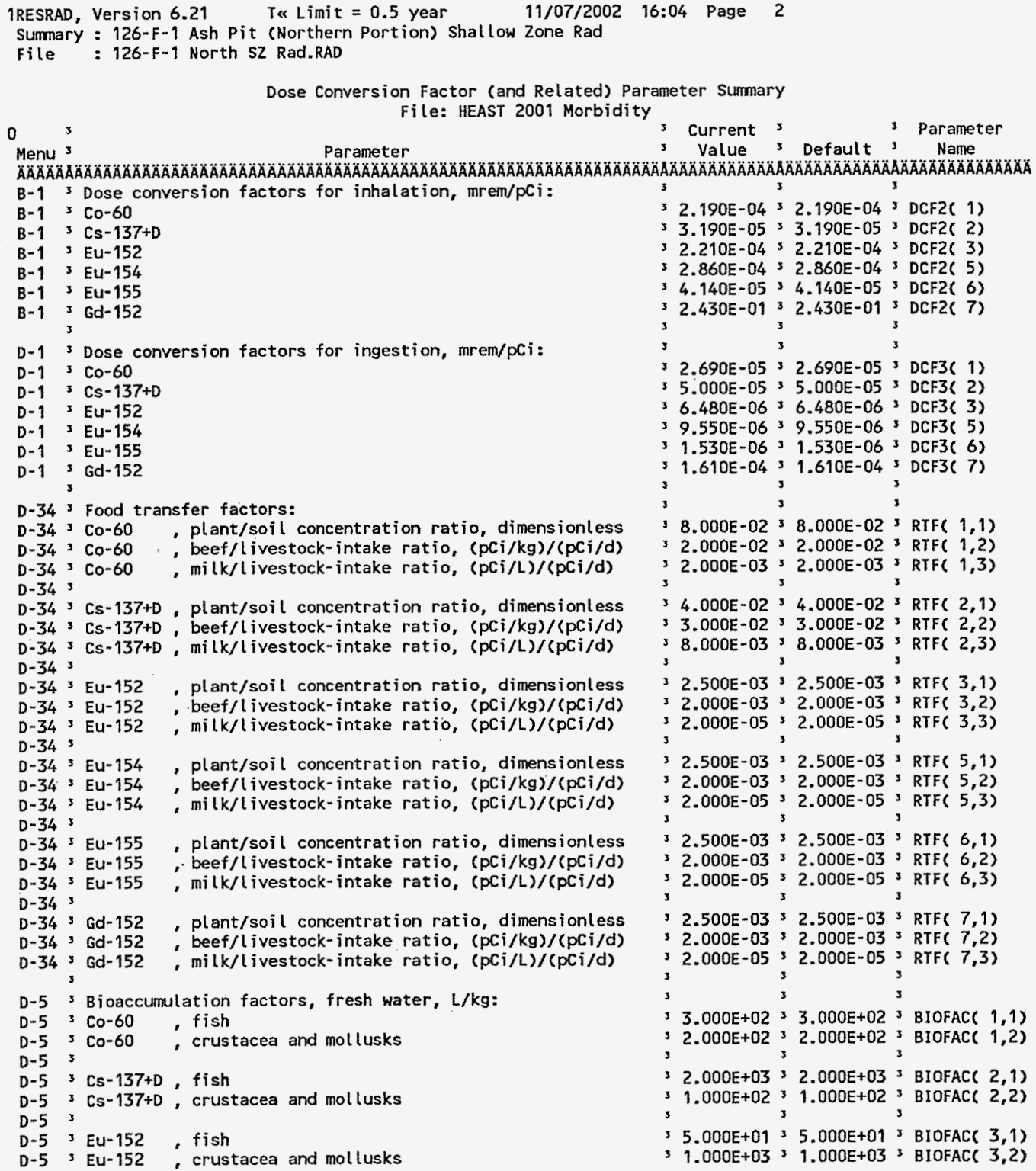

\begin{tabular}{|c|c|c|}
\hline $\begin{array}{l}\text { Attachment } \\
\text { riginator }\end{array}$ & I & Sheet No. 2 of $\underline{2 C}$ \\
\hline & $\begin{array}{l}\text { J.E. Thomson } \\
\text { S.W. Clark }\end{array}$ & Date \\
\hline
\end{tabular}




\section{ATTACHMENT 2}

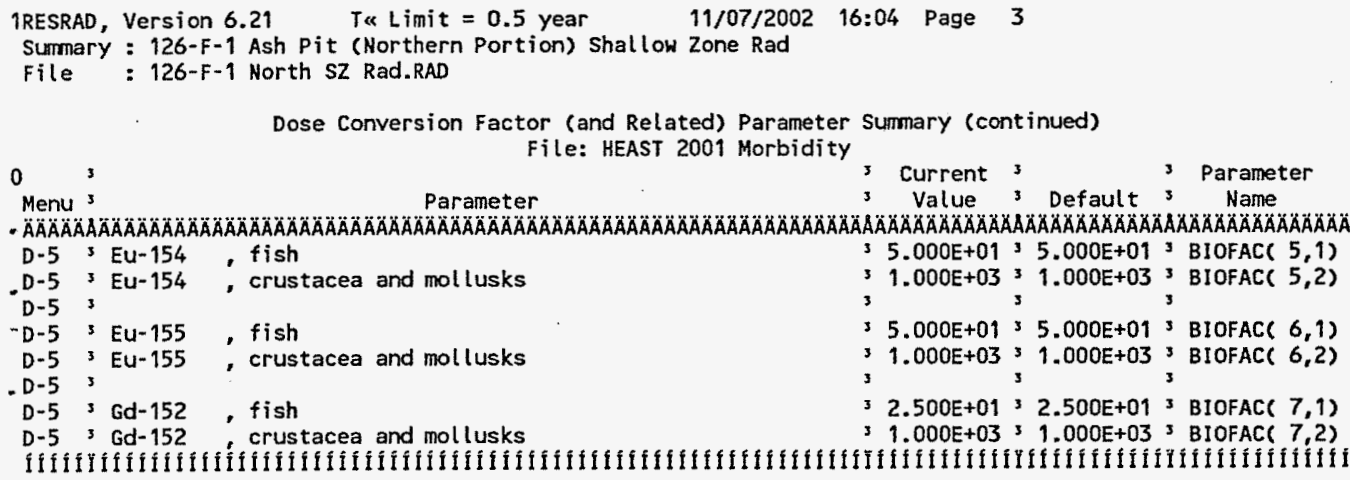

Attachment 2 Sheet No. 3 of $2 \mathrm{C}$ Originator J. E.Thomson Date 


\title{
ATTACHMENT 2
}

\begin{abstract}
IRESRAD, Version $6.21 \quad T \ll$ Limit $=0.5$ year
$11 / 07 / 2002 \quad 16: 04$ Page 4

Summary : 126-F-1 Ash Pit (Northern Portion) Shallow Zone Rad
\end{abstract}

File: 126-F-1 North SZ Rad.RAD

0

Site-Specific Parameter Sumary 3 User 3 3 , Used by RESRAD

Used by RESRAD 3 Parameter Menu 3 Parameter

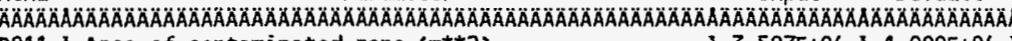
R011 3 Area of contaminated zone $\left(m^{* *} 2\right)$ RO11 Thickness of contaminated zone (m) R011' Length parallel to aquifer flow $(m)$ R011 Basic radiation dose limit (mrem/yr) R011 Time since placement of material (yr) R011' Times for calculations $(y r)$ R011 3 Times for calculations (yr) R011 s Times for calculations (yr) R011 Times for calculations (yr) R011 Times for calculations (yr) R011' Times for calculations (yr) R011' Times for calculations (yr) R011' Times for calculations (yr) R011' Times for calculations $(y r)$

$3.507 E+04^{3} 1.000 E+04$

$38.800 E+00 \div 2.000 E+00$

3 $3.150 E+02 ; 1.000 E+02$

$31.500 \mathrm{E}+0132.500 \mathrm{E}+01$

$30.000 E+00,0.000 E+00$

3 $1.000 E+0031.000 E+00$ s

$3.000 E+0033.000 E+00$

$7.000 E+00,1.000 E+01$

$1.600 E+0133.000 E+01$

$4.300 E+0131.000 E+02$

$1.000 E+0233.000 E+02$

$3.000 \mathrm{E}+0231.000 \mathrm{E}+03$

$1.000 E+0330.000 E+00$

3 not used $30.000 E+003$

R012 Initial principal radionuclide $(\mathrm{pCi} / \mathrm{g})=$ Co-60

$\begin{array}{lll}R 012 & 3 \text { Initial principal radionucl ide }(\mathrm{pCi} / \mathrm{g}): & \mathrm{Cs}_{\mathrm{s}}-137 \\ \mathrm{R} 012^{3} \text { Initial principal radionuclide }(\mathrm{pCi} / \mathrm{g}): & \text { Eu-152 }\end{array}$

$4.100 E-02>0.000 E+00$

3U.100E-02 3 0.000E+00

R012 3 Initial principal radionuclide $(\mathrm{pCi} / 9)$ : EU-155 $31.200 E-01 ; 0.000 E+00$,

$\mathrm{R}^{3}{ }^{3}$ Concentration in groundwater (PCi/L): $\mathrm{CO}^{-60} \mathrm{~B}^{3}$ not used $30.000 \mathrm{E}+00^{3}$

$\mathrm{R}^{3}{ }^{3}$ Concentration in groundwater (PCi/L): $\mathrm{Cs}_{2}-137 \mathrm{~s}^{3}$ not used $30.000 \mathrm{E}+00^{3}$

$R 012$ S Concentration in groundwater (pCi/L): Eu-152, not used $30.000 E+00$,

R012 Concentration in groundwater (pCi/L): Eu-154 ${ }^{3}$ not used $30.000 E+00$

R012 Concentration in groundwater $(\mathrm{pCi} / \mathrm{L})$ : Eu-155 3

R013' Cover depth (m)

R013 Density of cover material $\left(\mathrm{g} / \mathrm{cm}^{* * 3}\right)$

R013 3 Cover depth erosion rate $(\mathrm{m} / \mathrm{yr})$

R013 Density of contaminated zone $\left(\mathrm{g} / \mathrm{cm}^{* * 3}\right)$

R013' Contaminated zone erosion rate $(\mathrm{m} / \mathrm{yr})$

$\mathrm{R}^{3} 3^{3}$ Contaminated zone total porosity

R013 3 Contaminated zone field capacity

R013 Contaminated zone hydraulic conductivity (m/yr)

R013 Contaminated zone b parameter

R013 3 Average annual wind speed $(\mathrm{m} / \mathrm{sec})$

R013 3 Hunidity in air $\left(\mathrm{g} / \mathrm{m}^{*} * 3\right.$ )

R013 Evapotranspiration coefficient

R013 3 Precipitation $(m / y r)$

R013 3 Irrigation (m/ $\mathrm{yr}$ )

R013 J Irrigation mode

R013 3 Runoff coefficient

R013 3 Watershed area for nearby stream or pond $\left(m^{* * 2)}\right.$ R013' Accuracy for water/soil computations 3

R014 Density of saturated zone $\left(\mathrm{g} / \mathrm{cm}^{* * 3}\right)$

R014 Saturated zone total porosity

R014 S Saturated zone effective porosity

R014 Saturated zone field capacity

R014 3 Saturated zone hydraulic conductivity $(\mathrm{m} / \mathrm{yr})$
3

$30,000 \mathrm{E}+00^{3}$

not used $\$ 1.500 E+00$

not used $31.000 E-03$

$31.600 \mathrm{E}+0031.500 \mathrm{E}+00$

$1.000 E-03$ 3 $1.000 E-03$

$4.000 E-0134.000 E-01$ ?

$1.500 E-0132.000 E-013$

$2.500 E+0231.000 E+01$

$4.050 \mathrm{E}+00,5.300 \mathrm{E}+00$

$3.400 \mathrm{E}+0032.000 \mathrm{E}+00$

not used 3 8.000E+00

9.100E-01 $35.000 E-01$

$1.600 E-01>1.000 E+00$

$7.600 \mathrm{E}-01 \times 2.000 \mathrm{E}-01$

overhead 3 overhead

2.000E-01'2.000E-01

$1.000 E+06>1.000 E+06$

s $1.000 \mathrm{E}-03>1.000 \mathrm{E}-03$ s

$31.600 E+0031.500 E+00$

3 4. ODOE-013 $4.000 E-01$

$2.500 E-0132.000 E-01$

$31.500 \mathrm{E}-0132.000 \mathrm{E}-01$

$3.530 \mathrm{E}+03,1.000 \mathrm{E}+02$,

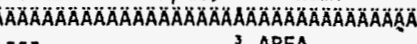

-.- 3 AREA

-.. 3 THICKO

.

...

...

-.. 3 T $3(3)$

$\cdots$

$3 T(5)$

-.. 3 T 6 ( 6$)$

... $3(7)$

$\begin{array}{ll}\ldots & 3 \\ \ldots & T\end{array}$

$-\cdots$

$\begin{array}{ll}\ldots . & 3 \\ \ldots & s 1(1) \\ \ldots & s 1(2)\end{array}$

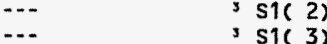

$3 \sin (5)$

... $351(6)$

-.. 3 W1( 1$)$

$\ldots \quad 3$ HIC 2$)$

3 Wi( 3 )

3 W1 5 )

-.. 3 W1( 6)

... 3 COVERO

-.. 3 DENSCV

$\cdots$

B DENSCZ

-.- $\quad$ VCZ

-.-

-.. 3 FCCZ

$3 \mathrm{HCCZ}$

$3 \mathrm{BCZ}$

-...

… HUMID

... 3 PRECIP

3 RI

3 IDITCH

-.. 3 RUNOFF

-..- 3 WAREA

-.- 3 EPS

3 DENSAO

-..

3 EPSZ

3 FCSZ

, HCSZ

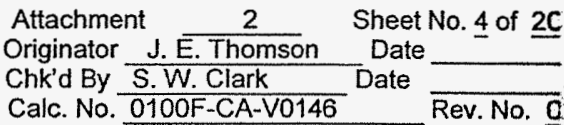




\section{ATTACHMENT 2}

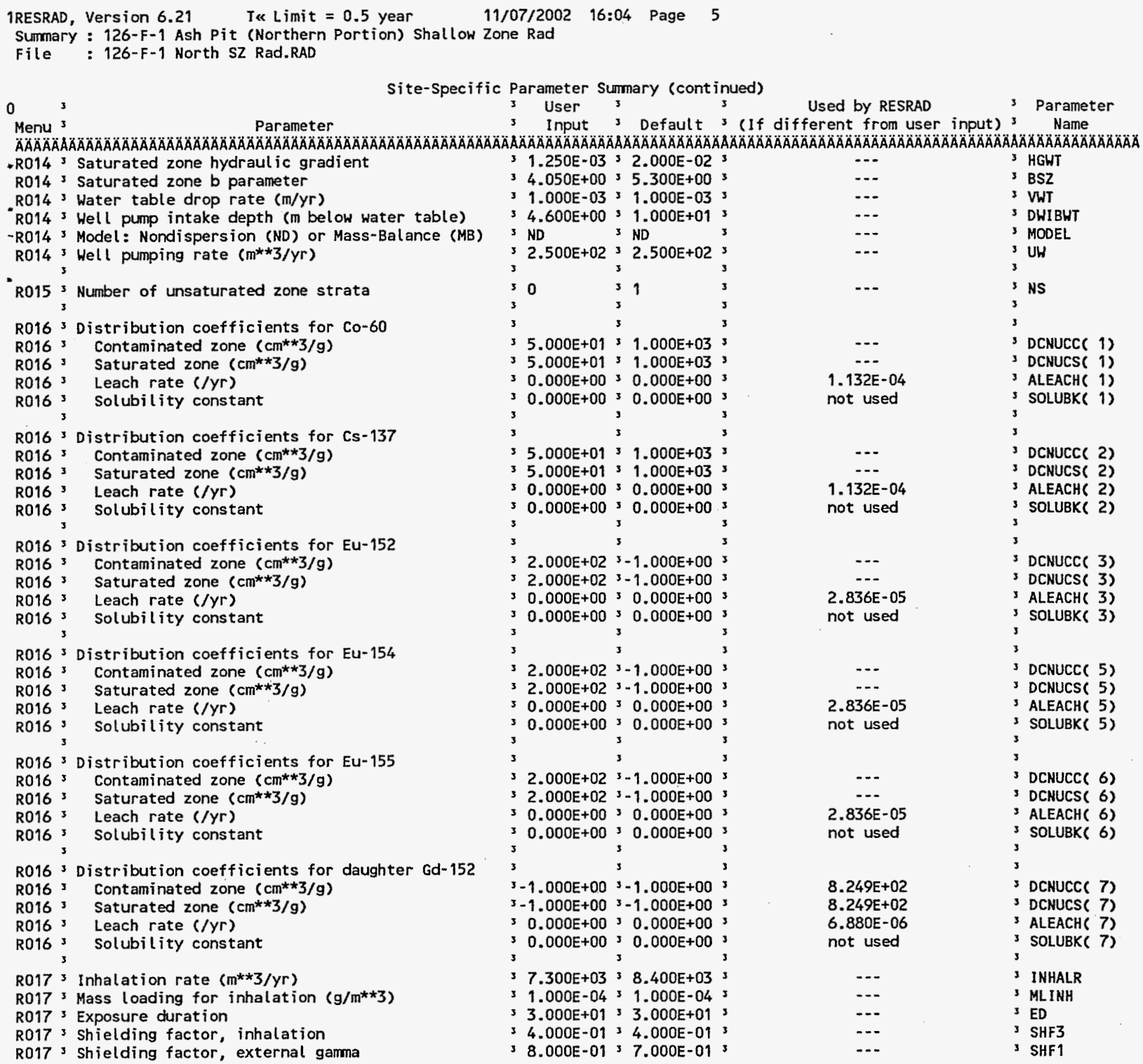

Attachment $\frac{2}{\text { Sheet No. } \underline{5} \text { of } \underline{2 C}}$
Originator J. E. Thomson Date
Chk'd By S.W. Clark
Calc. No. Date
O100F-CA-V0146




\section{ATTACHMENT 2}

1RESRAD, Version $6.21 \quad$ T« Limit $=0.5$ year $\quad \begin{array}{lll}11 / 07 / 2002 & 16: 04 & \text { Page } 6\end{array}$
Summary : $126-F-1$ Ash Pit (Northern Portion) Shallow Zone Rad

File : 126-F-1 North SZ Rad.RAD

\section{0}

Site-Specific Parameter Sumary (continued)

MenU $3^{3}$

\section{Parameter}

3 User ${ }_{3}$

Used by RESRAD 3 Parameter

Parameter

$\begin{array}{lll}R 0173 \text { Fraction of time spent indoors } & 36.000 E-0135.000 E-013 \\ R 0173 \text { Fraction of time spent outdoors (on site) } & 32.000 E-0132.500 E-013\end{array}$

R017 3 Fraction of time spent outdoors (on site)

R017 ${ }^{3}$ Shape factor flag, external gamma

R017' Radii of shape factor array (used if FS $=-1$ ):

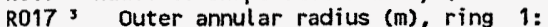

$\mathrm{R}_{017^{3}}{ }^{3}$ Outer annular radius (m), ring 2 :

$R 017^{3}$ Outer annular radius (m), ring 3

R017 ' Duter annular radius (m), ring 4:

R017 3 Duter annular radius (m), ring 5:

R017 3 Outer annular radius (m), ring $6:$

R017 3 Outer annular radius $(\mathrm{m})$, ring 7 :

$R_{017^{3}}{ }^{3}$ Outer annular radius $(m)$, ring 8 :

R017 ${ }^{3}$ Outer annular radius (m), ring 9:

R017 3 Duter annular radius $(m)$, ring 10:

$32.000 E-0132.500 E-013$

3 1.000E+00; $1.000 E+00$,

3 not used $35.000 E+01^{3}$

3 not used $37.071 E+013$

3 not used $30.000 E+00$;

3 not used $30.000 E+00$,

3 not used $30.000 E+00^{3}$

3 not used $30.000 E+00$ s

3 not used $30.000 E+00^{3}$

3 not used $0.000 \mathrm{E}+00 \mathrm{~J}$

not used $0.000 E+00^{3}$

3 not used $30.000 E+00^{3}$

3 not used $30.000 E+00^{3}$

R017 3 Outer annular radius (m), ring 11:

3 not used $30.000 E^{3}+00^{3}$

outer annular radius (m), ring 12:

R017 Fractions of annular areas within AREA:

R017 3 Ring 1

R017 : Ring 2

R017 s Ring 3

RO17 3 Ring 4

RO17 $^{3}$ Ring 5

R017 3 Ring 6

R017 3 Ring 7

R017 3 Ring 8

R017' Ring 9

R017 3 Ring 10

R017' Ring 11

R017 ${ }^{3}$ Ring 12

3 not used 's $1.000 \mathrm{E}+00^{3}$

not used 3 2.732E-01'

3 not used $30.000 E+00^{3}$

3 not used 3 O. O0OE+00 3

3 not used $30.000 E+00^{3}$

3 not used $30.000 E+00^{3}$

3 not used $30.000 E+00^{3}$

3 not used $30.000 E+00$ s

3 not used $30.000 E+00^{3}$

3 not used $30.000 E+00^{3}$

3 not used 3. $0.000 E+00^{3}$

3

3 FIND
F..

$>0$ shows circular AREA.

FotD

RAD SHAPE( 1)

-.. 3 RAD SHAPE( 2)

... 3 RAD_SHAPE( 3 )

-.. 3 RAD_SHAPE( 4)

-.. 3 RAD SHAPE( 5 )

-.. 3 RAD SHAPE( 6)

...

(... RAD_SHAPE( 8)

-.. TRADSHAPE( 9)

-.. S RAD SHAPE(10)

-.. 3 RAD SHAPE (11)

... RAD_SHAPE(12)

R018 Fruits, vegetables and grain consumption ( $\mathrm{kg} / \mathrm{yr}$ )

$R 018$, Leafy vegetable consumption $(\mathrm{kg} / \mathrm{yr})$, $2.700 \mathrm{E}+00>1.400 \mathrm{E}+013$

R018 ${ }^{3}$ Milk consumption ( $\mathrm{L} / \mathrm{Y} r$ )

$\mathrm{R}_{018}{ }^{3}$ Meat and poultry consumption $(\mathrm{kg} / \mathrm{yr})$

R018 3 Fish consumption $(\mathrm{kg} / \mathrm{yr})$

R018 3 Other seafood consumption $(\mathrm{kg} / \mathrm{yr})$

R018 s Soil ingestion rate $(g / y r)$

R018 3 Drinking water intake (L/Yr)

R018 3 Contamination fraction of drinking water

R018 3 Contamination fraction of household water

R018 3 Contamination fraction of livestock water

R018 \& Contamination fraction of irrigation water

R018 3 Contamination fraction of aquatic food

R018 S Contamination fraction of plant food

R018 Contamination fraction of meat

R018 3 Contamination fraction of milk

$R 019^{3}$ Livestock fodder intake for meat ( $\mathrm{kg} /$ day)

20193 Livestock fodder intake for milk ( $\mathrm{kg} /$ day)

$1.000 E+02,9.200 E+01^{3}$

$3.600 E+01,6.300 E+013$

$31.970 E+0135.400 E+00$ s

$39.000 E-0139.000 E-013$

$37.300 \mathrm{E}+01: 3.650 \mathrm{E}+013$

$3.300 E+02,5.100 E+02$

$1.000 E+00^{3} 1.000 E+00^{3}$

3 not used $1.000 E+00^{3}$

$31.000 E+00^{3} 1.000 E+00^{3}$

$31.000 E+0031.000 E+003$

3 5.000E-01 3.000 E-013

$3-1$

$3-1$

$3-1$

$3-1$

$36.800 E+01^{3} 6.800 E+01$

$35.500 \mathrm{E}+01^{3} 5.500 \mathrm{E}+01^{3}$

\begin{tabular}{|c|c|c|}
\hline & 2 & Sheet No. 6 of 20 \\
\hline & J. E. Thomson & Date \\
\hline $\begin{array}{l}\text { Chk'd By } \bar{S} \\
\text { Calc. No. } 0\end{array}$ & $\begin{array}{l}\text { W. Clark } \\
100 \mathrm{~F}-\mathrm{CA}-\mathrm{V} 014\end{array}$ & \\
\hline
\end{tabular}




\section{ATTACHMENT 2}

IRESRAD, Version 6.21 Tu Limit $=0.5$ year $\quad 11 / 07 / 2002 \quad 16: 04 \quad$ Page 7 Summary : 126-F-1 Ash Pit (Northern Portion) Shallow Zone Rad

File: 126-F-1 North SZ Rad.RAD

$0_{\text {Menu }}^{3}$

Site-Specific Parameter Summary (continued)

Parameter

3 User 3

3 Used by RESRAD

3 Parameter

A

R019' Livestock water intake for meat ( $\mathrm{L} /$ day)

$5.000 E+0135.000 E+01$

R019 Livestock water intake for milk (L/day)

$1.600 \mathrm{E}+02,1.600 \mathrm{E}+02,3$

3 5.000E-01 3 5.000E-013

RO193 Mass loading for fol iar deposition $\left(\mathrm{g} / \mathrm{m}^{* * 3}\right)$

$31.500 E-01,1.500 E-01$,

3 9.000E-0139.000E-013

? $1.000 E+0031.000 E+00$

3 not used $31.000 E+00^{3}$

$31.000 E+00>1.000 E+00$

R019 3 Drinking water fraction from ground water

R019 3 Household water fraction from ground water

$31.000 E+00$ s $1.000 E+00$ s

RO193 Irrigation fraction from ground water

3 7.000E-01 $37.000 \mathrm{E}-01$

$R 1983$ Wet weight crop yield for Non-Leafy $\left(\mathrm{kg} / \mathrm{m}^{\star *} 2\right)$

$R 19 B$ Wet weight crop yield for Leafy $\left(\mathrm{kg} / \mathrm{m}^{\star *} 2\right)$

$R 19 B$ B Wet weight crop yield for fodder $\left(\mathrm{kg} / \mathrm{m}^{* * 2}\right)$

R19B G Growing Season for Non-Leafy (years)

$31.500 \mathrm{E}+0031.500 \mathrm{E}+00$

$31.100 E+0031.100 E+00$

$31.700 \mathrm{E}-0131.700 \mathrm{E}-01$

$2.500 E-01>2.500 E-01$,

3 . $800 E-02 \div 8.000 E-02$

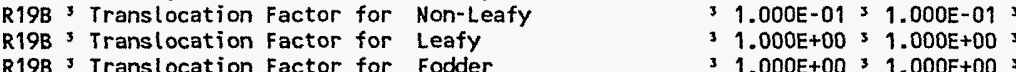

$\begin{array}{lll}\text { R19B } 3 \text { Translocation Factor for Non-Leafy } & 31.000 \mathrm{E}-0131.000 \mathrm{E}-01 \mathrm{~s} \\ \text { R19B } 3 \text { Translocation Factor for Leafy } & 31.000 \mathrm{E}+0031.000 \mathrm{E}+00 \mathrm{~s}\end{array}$

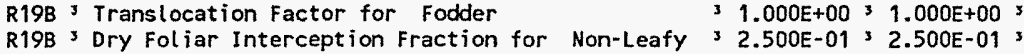

$R 19 B$ B Dry Foliar Interception Fraction for Leafy $32.500 E-0132.500 E-01$ ?

R19B Dry Foliar Interception Fraction for Fodder $32.500 E-0132.500 E-01$

R19B 3 Wet Foliar Interception Fraction for Non-Leafy $32.500 E-01^{3} 2.500 E-01$ s

$R 19 B 3$ Wet Foliar Interception Fraction for Leafy $32.500 E-0132.500 E-01$ s

R19B 3 Wet Foliar Interception Fraction for Fodder $32.500 E-0132.500 E-013$

R19B 3 Weathering Removal Constant for Vegetation $32.000 \mathrm{E}+01^{3} 2.000 \mathrm{E}+01$ s ,

C14 $3 \mathrm{C}-12$ concentration in water $\left(\mathrm{g} / \mathrm{cm}^{\star *}\right)$

C14 $3 \mathrm{C}-12$ concentration in contaminated soil $(\mathrm{g} / \mathrm{g})$

not used $32.000 \mathrm{E}^{3} 05$

3 not used 3 3.000E-02

3 not used $32.000 E-02$,

not used $3.800 \mathrm{E}-01$

3 not used 3 3.000E-01

not used 3 7.000E-07

3 not used $31.000 \mathrm{E}-10$

3 not used 38.000 E-013

not used $32.000 E-01$
not used $3.894 \mathrm{E}+01$

$143 \mathrm{c}-12$ evasion flux rate from soil $(1 / \mathrm{sec})$

C14 3 Fraction of grain in beef cattle feed

not used $38.894 \mathrm{E}+01_{3}^{3}$

C14 DCF correction factor for gaseous forms of $\mathrm{C14}$

3

STOR 3 Storage times of contaminated foodstuffs (days):

STOR ${ }^{3}$ Fruits, non-leafy vegetables, and grain

$31.400 E+0131.400 E+013$

$31.000 E+0031.000 E+003$

$3.000 E+00^{3} 1.000 E+00^{3}$

$2.000 E+01^{3} 2.000 E+01^{3}$

$7.000 E+00^{3}, 7.000 E+00^{3}$

$3.000 E+00 \div 7.000 E+00$

$31.000 E+0031.000 E+003$

$31.000 E+0031.000 E+00^{3}$

$34.500 E+0134.500 E+013$

\begin{tabular}{|c|c|c|}
\hline$\cdots$ & 3 & LWI5 \\
\hline$\cdots$ & 3 & LWI6 \\
\hline$\cdots$ & 3 & LSI \\
\hline -.. & 3 & MLFD \\
\hline$\cdots$ & 3 & DM \\
\hline$\cdots$ & 3 & DROOT \\
\hline$\cdots$ & $\mathbf{y}$ & FGWDW \\
\hline$\cdots$ & 3 & FGWHH \\
\hline$\cdots$ & 3 & FGWLW \\
\hline-- & $\begin{array}{l}3 \\
3\end{array}$ & FGWIR \\
\hline$\cdots$ & 3 & $Y V(1)$ \\
\hline$\cdots$ & 3 & YV(2) \\
\hline 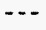 & s & YV(3) \\
\hline- & 3 & TE (1) \\
\hline$\cdots$ & 3 & TE (2) \\
\hline$\ldots$ & 3 & $T E(3)$ \\
\hline- & 3 & $\operatorname{TIV}(1)$ \\
\hline- & 3 & TIV(2) \\
\hline- & 3 & $\operatorname{TIV}(3)$ \\
\hline & 3 & RDRY (1) \\
\hline$\cdots$ & 3 & RDRY (2) \\
\hline- & 3 & RDRY (3) \\
\hline.- & 3 & RWET(1) \\
\hline- & 3 & RWET (2) \\
\hline - & 3 & RWET (3) \\
\hline- & 3 & WLAM \\
\hline$\ldots$ & 3 & \\
\hline & 3 & C12WTR \\
\hline$\ldots$ & 3 & $\begin{array}{l}\text { C12C2 } \\
\text { CSOIL }\end{array}$ \\
\hline & 3 & CAIR \\
\hline & 3 & DMC \\
\hline.- & 3 & EVSN \\
\hline-1 & 3 & REVSN \\
\hline$\cdots$ & 3 & AVFG4 \\
\hline & s & AVFG5 \\
\hline & 3 & $\mathrm{CO} 2 \mathrm{~F}$ \\
\hline & 3 & \\
\hline & 3 & STOR_T(1) \\
\hline & 3 & STOR_T(2) \\
\hline & 3 & STOR_T(3) \\
\hline & 3 & STOR_T(4) \\
\hline & 3 & STOR_T(5) \\
\hline & 3 & STOR_T(6) \\
\hline & 3 & STOR_T(7) \\
\hline & 3 & STOR_T( 8$)$ \\
\hline & 3 & STOR_T(9) \\
\hline
\end{tabular}

Attachment

Originator J. E. Thomson

Sheet No. 7 of $2 c$

Chk'd By S.W. Clark

Date

Calc. No. 0100F-CA-V0146

Date

Rev. No. (




\section{ATTACHMENT 2}

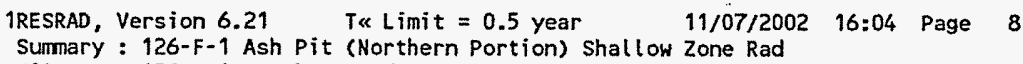

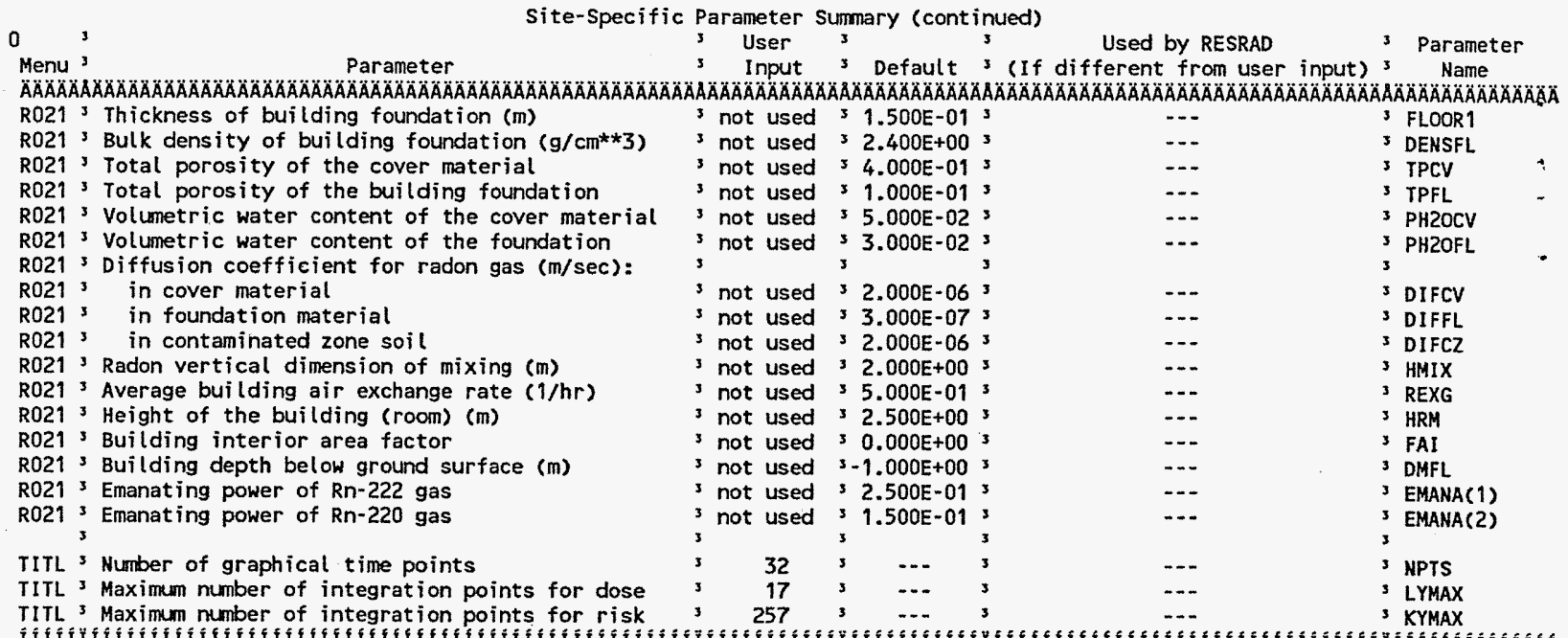

Summary of Pathway Selections

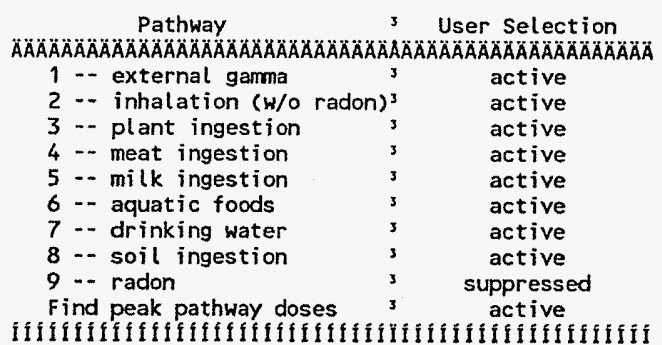

Attachment $\frac{2}{2}$ Sheet No. 8 of $\underline{2 C}$ Originator J. E.Thomson Date Chk'd By S.W. Clark Date

Calc. No. O100F-CA-V0146 


\section{ATTACHMENT 2}

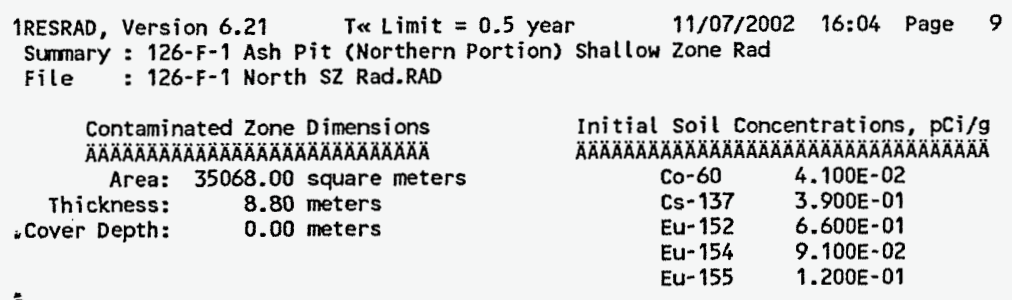

o

Total Dose TDOSE(t), mrem/yr

Basic Radiation Dose Limit $=1.500 \mathrm{E}+01 \mathrm{mrem} / \mathrm{yr}$

Total Mixture Sum $M(t)=$ Fraction of Basic Dose Limit Received at Time $(t)$

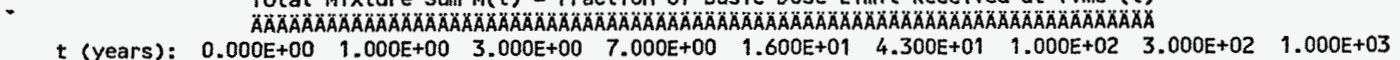

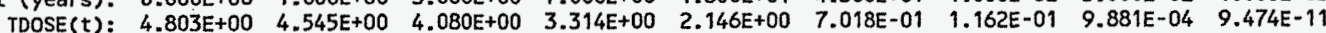

$M(t): \quad 3.202 E-01 \quad 3.030 E-01 \quad 2.720 E-01 \quad 2.210 E-01 \quad 1.431 E-01 \quad 4.679 E-02 \quad 7.750 E-03 \quad 6.587 E-05 \quad 6.316 E-12$

OMaximum TDOSE $(t): 4.803 E+00 \mathrm{mrem} / \mathrm{yr}$ at $\mathrm{t}=0.000 \mathrm{E}+00$ years

Attachment

2

Sheet No. 9 of $2 C$

Originator J. E.Thomso

Calc. No. O100F-CA-V0146

Rev. No. $\mathrm{C}$ 


\section{CALCULATION COVER SHEET}

Project Title 126-F-1 Cleanup Verification

Job No. 14655

Area 100-F Area

Discipline Environmental *Calc. No.

0100F-CA-V0102

Subject 126-F-1 (Southern Portion) RESRAD Calculation

Computer Program RESRAD

Program No. Version 6.3

The attached calculations have been generated to document compliance with established cleanup levels.

These calculations should be used in conjunction with other relevant documents in the administrative record.

\section{Committed Calculation $\otimes \quad$ Preliminary $\square \quad$ Superseded $\square \quad$ Voided}

\begin{tabular}{|c|c|c|c|c|c|c|}
\hline Rev. & Sheet Numbers & Originator & Checker & Reviewer & Approval & Date \\
\hline 0 & $\begin{array}{l}\text { Cover }-1 \mathrm{pg} \\
\text { Summary }-4 \mathrm{pg} \\
\text { Attm. } 1-1 \mathrm{pg} \\
\text { Attm. } 2-20 \mathrm{pg} \\
\text { Attm. } 3-22 \mathrm{pg} \\
\text { Attm. } 4-11 \mathrm{pg} \\
\text { Total }-59 \text { pages }\end{array}$ & $\begin{array}{c}\text { T. M. Routt } \\
\text { Approved } \\
\text { 09/05/01 }\end{array}$ & $\begin{array}{c}\text { S. W. Clark } \\
\text { Approved } \\
\text { 09/05/01 }\end{array}$ & $\begin{array}{l}\text { K. E. Cook } \\
\text { Approved } \\
\text { 09/05/01 }\end{array}$ & $\begin{array}{c}\text { M. A. Buckmaster } \\
\text { Approved } \\
09 / 10 / 01\end{array}$ & $\begin{array}{c}\text { Approved } \\
09 / 10 / 01\end{array}$ \\
\hline 1 & 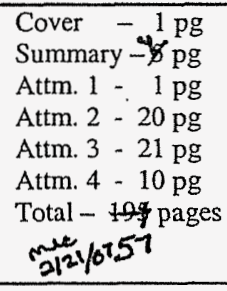 & $\begin{array}{l}\text { S. W. Clark } \\
\text { 85w sane } \\
2 / 12 / 07\end{array}$ & $\begin{array}{l}\text { M. W. Perrott } \\
\text { M/e/Pettot } \\
2 / 13 / 07\end{array}$ & N/A & S. W. Callison & $2-20-07$ \\
\hline \multicolumn{7}{|c|}{ SUMMARY OF REVISION } \\
\hline 1 & \multicolumn{6}{|c|}{$\begin{array}{l}\text { This calculation brief was revised to account for radioactive decay since the original calculation (Rev. } 0 \text { ) was } \\
\text { performed in } 2001 \text {. For convenience the entire calculation brief including the cover sheet has been replaced. }\end{array}$} \\
\hline & & & & & & \\
\hline & & & & & & \\
\hline
\end{tabular}


Washington Closure Hanford CALCULATION SHEET

\begin{tabular}{|c|c|c|c|c|c|c|}
\hline Originator: & S. W. Clark \& & Date: & $3 / 13 / 0>$ Calc. No.: & 0100F-CA-V0102 & Rev.: & \\
\hline
\end{tabular}

\section{PURPOSE:}

2
Calculate the soil and groundwater concentrations, dose, and risk contributions from remaining radionuclide contaminants in vadose zone soil at the southern portion of the 126-F-1 Powerhouse Ash Pit over a period of 1,000 years.

\section{GIVEN/REFERENCES:}

1) Cleanup verification data from $126-F-1$ (Southern Portion) Cleanup Verification $95 \%$ UCL Calculation, Calculation No. 0100F-CA-V0100, Rev. 0, Bechtel Hanford, Inc., Richland, Washington.

2) Remedial Design Report/Remedial Action Work Plan for the 100 Area (RDR/RAWP), DOE/RL-96-17, Rev. 5, U.S. Department of Energy, Richland Operations Office, Richland, Washington.

3) Radioactive and nonradioactive contaminants of potential concern (COPCs) from the 100 Area Remedial Action Sampling and Analysis Plan, DOE/RL-96-22, Rev. 2, U.S.

Department of Energy, Richland Operations Office, Richland, Washington. For the purpose of these RESRAD calculations, the radioactive COCs were cesium-137, cobalt- 60 , europium-152, europium-154, and europium-155. There were no nonradionuclide COCs.

4) RESidual RADioactivity (RESRAD) computer code, version 6.3, to calculate compliance with residual radioactivity guidelines, developed for the U.S. Department of Energy by the Environmental Assessment Division of Argonne National Laboratory, Argonne, Illinois.

5) Sample design data from the Sampling and Analysis Instruction for Confirmatory Sampling of the Southern Half of the 126-F-1 Ash Pit, BHI-01522, Rev. 1, Bechtel Hanford, Inc., Richland, Washington.

6) 100-F Remedial Action Sampling Logbook EL-1535-1.

\section{SOLUTION:}

1) A single RESRAD run was performed for the entire vadose zone soil horizon, applying shallow zone cleanup verification criteria. Table 1 shows the elevations (NAVD88), thickness, and areal dimensions of the soil horizon. Attachment 1 shows dimensions of the soil horizon and contaminant pathways considered for dose, risk, and groundwater protection modeling. Input factors for each run are shown in the "Summary" section of the RESRAD "Mixture Sums and Single Radionuclide Guidelines" printouts in the Attachments to this Calculation Summary. 
Washington Closure Hanford

CALCULATION SHEET Originator: S. W. Clark ot.

\begin{tabular}{l|l|l|l|} 
Project: & 126-F-1 Cleanup Verification & Job No: & 14655 \\
\hline Subject: & 126-F-1 (Southern Portion) RESRAD Calculation
\end{tabular} Date: 010 Calc No:

Checked:

0100F-CA-V0102

D Calculation

Table 1. Waste Site Dimensions for RESRAD Modeling

\begin{tabular}{|l|c|c|}
\hline \multicolumn{1}{|c|}{ Parameter } & Units & $\begin{array}{c}\text { Contaminated Zone } \\
\text { Dimensions }\end{array}$ \\
\hline Cover Depth & $\mathrm{m}$ & 0 \\
\hline Area of Contaminated Zone (CZ) & $\mathrm{m}^{2}$ & 72,840 \\
\hline Length Parallel to Aquifer Flow & $\mathrm{m}$ & 353 \\
\hline Elevation: Surface & $\mathrm{m}$ & 122.0 \\
\hline Elevation: Groundwater & $\mathrm{m}$ & 115.0 \\
\hline Thickness: Contaminated Zone & $\mathrm{m}$ & 7.0 \\
\hline
\end{tabular}

1
2) The cleanup verification data set for the southern portion of the 126-F-1 Powerhouse Ash Pit is presented in Table 2. The year where the peak dose (or concentration) occurs from each individual radionuclide contaminant of concern (COC) and layer is determined by a preliminary run. This year is then added for all horizons for the final RESRAD runs. For the direct exposure pathway (i.e. soil ingestion and inhalation and external radiation), the peak year occurred at year zero (year 2007) for all COCs. RESRAD evaluations were performed for the southern portion of the 126-F-1 Powerhouse Ash Pit assuming that the residual contamination levels from the shallow zone sample data sets extend to groundwater, $7 \mathrm{~m}$ (23 ft) below the ground surface (Attachment 1). For the water pathways (i.e. drinking water and food ingestion) the peak year was year 7 for cobalt-60, year 19 for europium-152, year 12 for europium-154, and year 10 for europium-155. The 7, 10, 12, and 19-year time periods were added to all RESRAD runs.

\begin{tabular}{|l|c|c|}
\hline \multicolumn{3}{|c|}{ Table 2. Cleanup Verification Data Set } \\
\hline Contaminants of Concern & $\begin{array}{c}\text { Radionuclide Activity } \\
\text { When Sampled (5/21/2001), } \\
\text { (pCi/g) }\end{array}$ & $\begin{array}{c}\text { Radionuclide Activity }_{\text {Decayed to 2/8/2007, }} \\
\text { (pCi/g) }\end{array}$ \\
\hline Cesium-137 & $0(<\mathrm{BG})$ & $0(<\mathrm{BG})$ \\
\hline Cobalt-60 & 0.26 & 0.12 \\
\hline Europium-152 & 3.5 & 2.6 \\
\hline Europium-154 & 0.42 & 0.27 \\
\hline Europium-155 & 0.061 & 0.027 \\
\hline $\begin{array}{l}\text { a Soil concentration values from 126-F-1 (Southern Portion) Cleanup Verification 95\% UCL Calculation, } \\
\text { Calculation No. 0100F-CA-V0102, Rev. 0, Bechtel Hanford, Inc., Richland, Washington are reduced by } \\
\text { radioactive decay calculations from original sample values on 5/21/2001 to present values (2/8/2007). }\end{array}$ \\
\hline
\end{tabular}

\section{METHODOLOGY:}

1) Runs of RESRAD version 6.3 were completed for the southern portion of the 126-F-1 Powerhouse Ash Pit using the radionuclide concentrations shown in Table 2. RESRAD numerical output reports for dose, risk, and concentration are presented in the Attachments to this calculation summary. 
Washington Closure Hanford Originator: S. W. Clark 2ke Project: $126-\mathrm{F}-1$ Cleanup Verification Subject: $126-\mathrm{F}-1$ (Southern Portion) RESRAD Calculation

\section{CALCULATION SHEET}

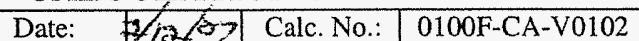

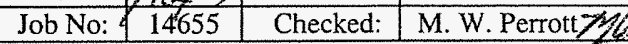

M. W. Perrott/

20

Rev.

Date: $213,1,3,2$

Sheet $\mathrm{No}_{3}$ of 4
1

2

3

4

5

6

7

8

9

10

14

15

16

17

18

19

20

21

22

23

24

25

26

27

\section{RESULTS:}

\section{1) Radionuclide "All Pathways" Dose Rate}

The "all pathways" (maximum) dose rates are shown in Table 3. The maximum all pathways dose rate from the southern portion of the 126-F-1 Powerhouse Ash Pit is $14.3 \mathrm{mrem} / \mathrm{yr}$ at year zero (2007).

\begin{tabular}{|c|c|c|c|c|c|c|c|c|c|}
\hline \multicolumn{8}{|c|}{ Table 3. All Pathways Dose Rate (mrem/yr) } \\
\hline $\begin{array}{c}\text { Vadose Zone } \\
\text { Horizon }\end{array}$ & \multicolumn{7}{|c|}{ "All Pathways" Dose Contributions in mrem/yr at Each Time Slice (yr) } \\
\cline { 2 - 9 } & 0 & 1 & 7 & 10 & 11 & 12 & 19 & 300 & 1000 \\
\hline Shallow Zone & $1.43 \mathrm{E}+01$ & $1.35 \mathrm{E}+01$ & $9.41 \mathrm{E}+00$ & $7.91 \mathrm{E}+00$ & $7.47 \mathrm{E}+00$ & $7.05 \mathrm{E}+00$ & $4.76 \mathrm{E}+00$ & $1.95 \mathrm{E}-06$ & $4.94 \mathrm{E}-15$ \\
\hline
\end{tabular}

\section{2) Radionuclide Excess Lifetime Cancer Risk}

The radionuclide excess lifetime cancer risk (ELCR) results are shown in Table 4. The maximum ELCR for the southern portion of the 126-F-1 Powerhouse Ash Pit is $1.58 \times 10^{-4}$ at year zero (2007).

\begin{tabular}{|c|c|c|c|c|c|c|c|c|c|}
\hline & Ta & e 4. & & de exce & & & 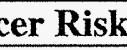 & & \\
\hline \multirow{2}{*}{$\begin{array}{c}\text { Vadose Zone } \\
\text { Horizon }\end{array}$} & \multicolumn{9}{|c|}{ Excess Cancer Risk at Each Time Slice (yr) } \\
\hline & 0 & 1 & 7 & 10 & 11 & 12 & 19 & 300 & 1000 \\
\hline Shallow Zone & $1.58 \mathrm{E}-04$ & $1.49 \mathrm{E}-04$ & $1.06 \mathrm{E}-04$ & $8.98 \mathrm{E}-05$ & $8.49 \mathrm{E}-05$ & $8.04 \mathrm{E}-05$ & $5.48 \mathrm{E}-05$ & $2.30 \mathrm{E}-11$ & $3.24 \mathrm{E}-20$ \\
\hline
\end{tabular}

\section{3) Radionuclide Groundwater Protection}

The radionuclide concentrations in groundwater calculated by the RESRAD model are summarized in Table 5. The organ specific dose via the groundwater (and river) pathway is calculated in a separate calculation brief. Only concentrations are presented here.

\begin{tabular}{|c|c|c|c|c|c|c|c|c|c|c|}
\hline \multirow{2}{*}{$\begin{array}{l}\text { Radio- } \\
\text { nuclides }\end{array}$} & \multicolumn{9}{|c|}{ Groundwater Concentrations in $\mathrm{pCi} / \mathrm{L}$ at Each Time Slice (yr) } & \multirow{2}{*}{$\begin{array}{l}\text { RAGs } \\
\mathrm{pCi} / \mathrm{L}\end{array}$} \\
\hline & 0 & 1 & 7 & 10 & 11 & 12 & 19 & 300 & 1000 & \\
\hline Co-60 & 0 & $7.26 \mathrm{E}-04$ & $2.31 \mathrm{E}-03$ & $2.22 \mathrm{E}-03$ & $2.14 \mathrm{E}-03$ & $2.05 \mathrm{E}-03$ & $1.29 \mathrm{E}-03$ & $1.75 \mathrm{E}-18$ & 0 & 100 \\
\hline $\mathrm{Eu}-152$ & 0 & 1.07E-03 & $5.48 \mathrm{E}-03$ & $6.70 \mathrm{E}-03$ & $6.99 \mathrm{E}-03$ & $7.24 \mathrm{E}-03$ & $7.96 \mathrm{E}-03$ & $5.53 \mathrm{E}-08$ & $2.69 \mathrm{E}-23$ & 200 \\
\hline Eu-154 & 0 & $1.08 \mathrm{E}-04$ & $4.72 \mathrm{E}-04$ & 5.32E-04 & $5.41 \mathrm{E}-04$ & $5.45 \mathrm{E}-04$ & $4.97 \mathrm{E}-04$ & $1.87 \mathrm{E}-12$ & $4.53 \mathrm{E}-36$ & 60 \\
\hline Eu-155 & 0 & $1.02 \mathrm{E}-05$ & $3.08 \mathrm{E}-05$ & $2.89 \mathrm{E}-05$ & $2.77 \mathrm{E}-05$ & $2.62 \mathrm{E}-05$ & $1.56 \mathrm{E}-05$ & $2.12 \mathrm{E}-21$ & 0 & 600 \\
\hline
\end{tabular}

\section{CONCLUSIONS:}

- The "all pathways" (maximum) dose rates are shown in Table 3. The maximum all pathways dose rate from the southern portion of the $126-\mathrm{F}-1$ Powerhouse Ash Pit is $14.3 \mathrm{mrem} / \mathrm{yr}$ at year zero (2007). 
Washington Closure Hanford \begin{tabular}{|l|l|l|}
\hline Originator: & S. W. Clark $2 . / 5$ \\
\hline
\end{tabular}

\begin{tabular}{l|l|l|l} 
Project: & $126-\mathrm{F}-1$ Cleanup Verification & Job No: & $1 A$ \\
Subject: & $126-\mathrm{F}-1$ (Southern Portion) RESRAD Calculation
\end{tabular} CALCULATION SHEET TION SHEET

Subject: $126-\mathrm{F}-1$ (Southern Portion) RESRAD Calculation

- None of the site COCs are projected to exceed remedial action goals (RAGs).

- The radionuclide excess lifetime cancer risk (ELCR) results are shown in Table 4. The maximum ELCR for the southern portion of the 126-F-1 Powerhouse Ash Pit is $1.58 \times 10^{-4}$ at year zero (2007).

- The dominant pathway for the dose rate is direct external exposure.

- The primary radionuclide contributing to the direct exposure pathway is europium-152.

- Among the radionuclide contaminants of concern, cobalt-60, europium-152, europium-154, and europium-155 are calculated to reach groundwater in the 1,000 years of the RESRAD model run. These radionuclides are predicted to reach groundwater at concentrations greatly below the RAGs for drinking water consumption.

\section{ATTACHMENTS:}

1. Graphic showing 126-F-1 Ash Pit Southern Portion Cleanup Verification Model (1 page)

2. RESRAD Output: 126-F-1 Ash Pit Southern Portion Radionuclides - Mixture Sums and Single Radionuclide Guidelines (20 pages)

3. RESRAD Output: 126-F-1 Ash Pit Southern Portion Radionuclides - Intake Quantities and Health Risk Factors (21 pages)

4. RESRAD Output: 126-F-1 Ash Pit Southern Portion Radionuclides - Concentration of Radionuclides, (10 Pages) 
126-F-1 Ash Pit Southern Portion Cleanup Verification Model

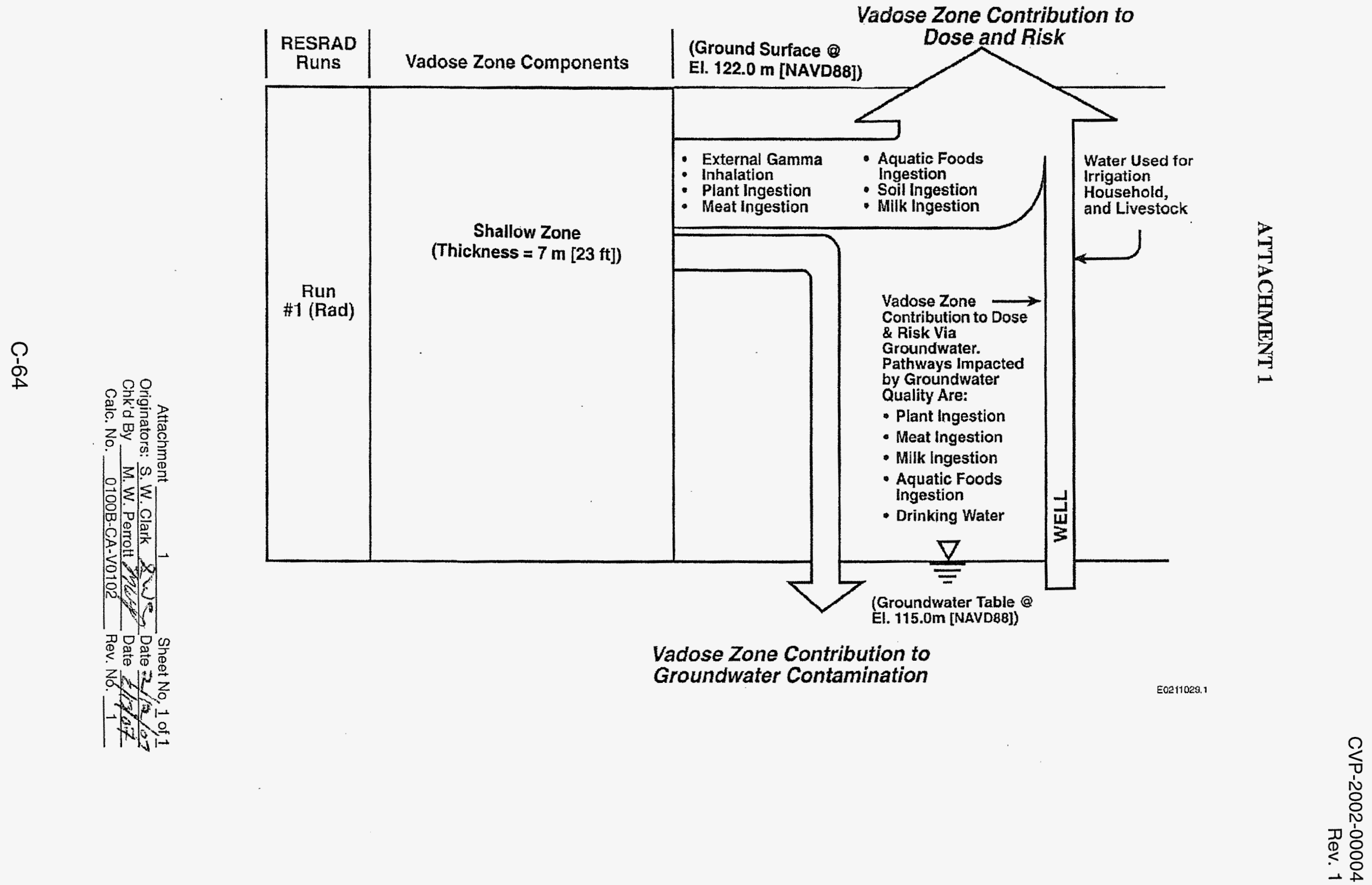




\section{ATTACHMENT 2}

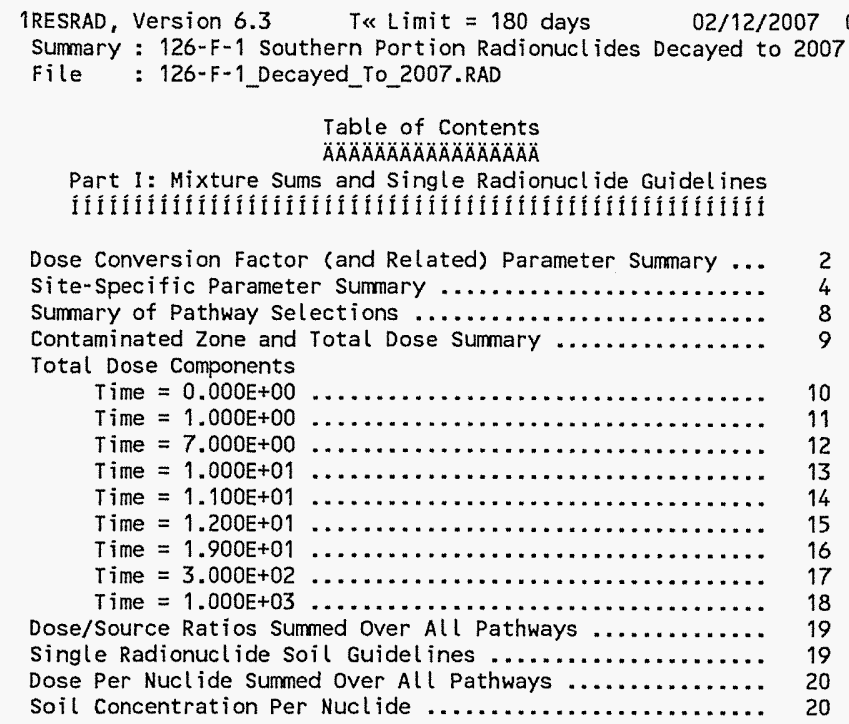

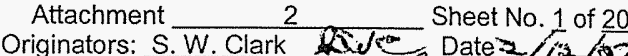
Chk'd By MW Perrott M Date $2 / \%$

Calc. No. 0100B-CA-V0102 Rev. No. 1 


\section{ATTACHMENT 2}

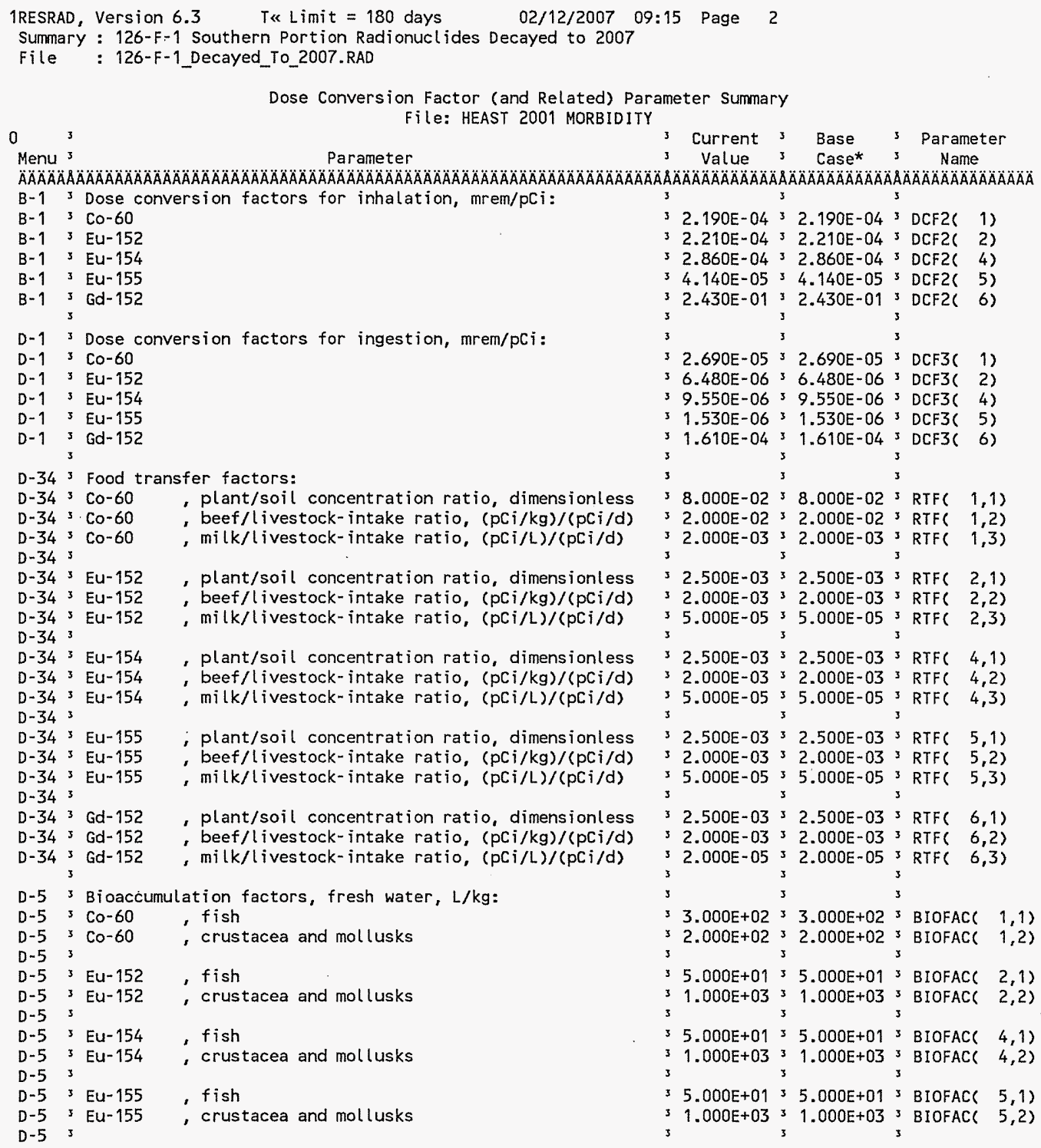

\begin{tabular}{|c|c|c|}
\hline \multicolumn{2}{|c|}{ Attachment $\quad 2$} & \multirow{2}{*}{$\begin{array}{l}\text { Sheet No. } 2 \text { of } \underline{20} \\
\text { Date }\end{array}$} \\
\hline Originators: & S. W. Clark & \\
\hline Chk'c & M. W. Perrott & Date \\
\hline Calc & 0100B-CA-V0102 & Rev. No. \\
\hline
\end{tabular}




\section{ATTACHMENT 2}

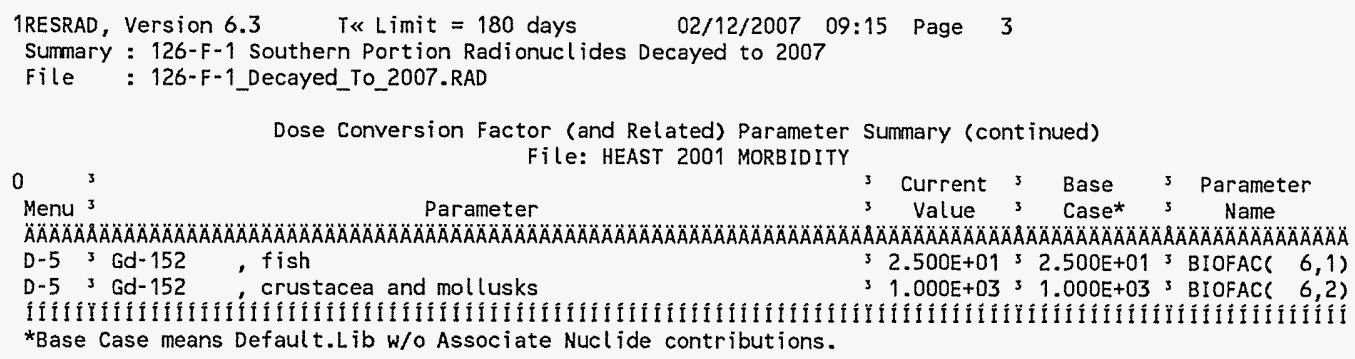




\section{ATTACHMENT 2}

1RESRAD, Version 6.3 T\& Limit $=180$ days $02 / 12 / 2007 \quad 09: 15$ Page 4 Summary : 126-F-1 Southern Portion Radionuclides Decayed to 2007

File : 126-F-1_Decayed_To_2007.RAD

0 Menu 3

Site-Specific Parameter Sumary

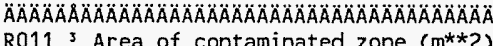
R011 Thickness of contaminated zone (m)

R011 Length parallel to aquifer flow (m)

R011'Basic radiation dose limit (mrem/yr)

R011 Time since placement of material (yr)

R011 ' Times for calculations $(y r)$

R011 3 Times for calculations ( $y r$ )

R011 3 Times for calculations (yr)

R011' Times for calculations (yr)

R011 Times for calculations (yr)

R011 ' Times for calculations (yr)

R011 3 Times for calculations (yr)

R011 Times for calculations (yr)

R011' Times for calculations (yr) 3

$\begin{array}{lll}3 & \text { User }{ }_{3}^{3} & \\ 3 & \text { Input } 3 \text { Default }\end{array}$

Used by RESTRAD I Parameter 3 Input ${ }^{3}$ Default ${ }^{3}$ (If different from user input) ${ }^{3}$ Name $37.284 \mathrm{E}+04,1.000 \mathrm{E}+04 ; 3.0003$ AREA

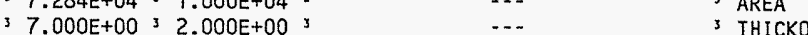
$33.530 E+0231.000 E+02$ 3 3 -.. $31.500 E+01^{3} 3.000 E+01^{3} \quad \ldots . .3$ BRDL $30.000 E+00^{3} 0.000 E+00^{3} \quad \ldots . .25$ $31.000 E+0031.000 E+003$ $37.000 E+0033.000 E+003$ $31.000 E+0131.000 E+013$ $3 \begin{array}{lll}1.000 E+01 & 3.000 E+01 & 3 \\ 3 & 1.100 E+01 & 3.000 E+01\end{array}$ $31.200 E+0131.000 E+023$ $31.900 E+0133.000 E+023$ $33.000 E+02,1.000 E+033$ $31.000 E+03,0.000 E+003$ 3 not used $30.000 E+00_{3}^{3}$ R012 Initial principal radionuclide $(\mathrm{pCj} / \mathrm{g})$ : Co-60 $31.200 \mathrm{E}-01^{3} 0.000 \mathrm{E}+00^{3}$ R012 Initial principal radionuclide $(\mathrm{pCi} / \mathrm{g}):$ Eu-152 $32.600 \mathrm{E}+00^{3} 0.000 \mathrm{E}+00^{3}$ $\mathrm{R}_{012^{3}}{ }^{3}$ Initial principal radionuclide $(\mathrm{pCi} / \mathrm{g}):$ Eu-154 $32.700 \mathrm{E}-0130.000 \mathrm{E}+00$ $\mathrm{R}_{012}{ }^{3}$ Initial principal radionuclide $(\mathrm{pCi} / \mathrm{g})$ : $\mathrm{Eu}-155,32.700 \mathrm{E}-02^{3} 0.000 \mathrm{E}+00$, $\mathrm{R} 012^{3}$ Concentration in groundwater ( $\mathrm{PCi} / \mathrm{L}$ ): $\mathrm{CO}-60$ s not used $30.000 \mathrm{E}+0 \mathrm{O}^{\mathrm{s}}$ R012 'Concentration in groundwater ( $\mathrm{pCi} / \mathrm{L})$ : EU-152 3 not used $30.000 E+00$ s R012 3 Concentration in groundwater ( $\mathrm{PC} / \mathrm{L}$ ): EU-154's not used $30.000 E+00^{3}$

$\mathrm{R}^{3}$, Concentration in groundwater ( $\left.\mathrm{pCi} / \mathrm{L}\right)$ : Eu-155, not used $3_{3}^{3} 0.000 \mathrm{E}+00_{3}^{3}$ 3

R013 3 Cover depth (m)

R013 3 Density of cover material $\left(\mathrm{g} / \mathrm{cm}^{* *} 3\right)$

R013 Cover depth erosion rate $(\mathrm{m} / \mathrm{yr})$

R013 Density of contaminated zone $(\mathrm{g} / \mathrm{cm} * * 3)$

R013 3 Contaminated zone erosion rate $(\mathrm{m} / \mathrm{yr})$

R013 Contaminated zone total porosity

R013 3 Contaminated zone field capacity

$30.000 \mathrm{E}+00^{3} 0.000 \mathrm{E}+00^{3}$

3 not used $31.500 E+00$ s

3 not used $31.000 E-03$ s

$31.600 E+0031.500 E+003$

$31.000 E-03 ; 1.000 E-03$ s

3 4.000E-01' 4.000E-013

$31.500 \mathrm{E}-01$ 3 $2.000 \mathrm{E}-01$ s

R013 3 Contaminated zone hydraulic conductivity $(\mathrm{m} / \mathrm{Yr}) 32.500 \mathrm{E}+0231.000 \mathrm{E}+01$ s

R013 3 Contaminated zone b parameter

R013 Average annul wind speed $(\mathrm{m} / \mathrm{sec})$

$34.050 \mathrm{E}+0035.300 \mathrm{E}+00$;

3 $3.400 E+00>2.000 E+00$ s

not used $38.000 E+00^{3}$

9.100E-013 $5.000 E-01^{3}$

$31.600 E-01^{3} 1.000 E+00^{3}$

$37.600 E-01^{3} 2.000 E-013$

3 overhead 3 overhead

$32.000 E-0132.000 E-013$

R013 3 Irrigation $(\mathrm{m} / \mathrm{yr})$

R013 I Irrigation mode

R013 Sunoff coefficient

$31.000 E+0631.000 E+063$

Watershed area for nearby stream or pond $\left(\mathrm{m}^{* * 2}\right.$ 3

R014 3 Density of saturated zone $\left(\mathrm{g} / \mathrm{cm}^{* * 3}\right)$

R014 S Saturated zone total porosity

R014 S Saturated zone effective porosity

R014 3 Saturated zone field capacity

R014 3 Saturated zone hydraulic conductivity $(\mathrm{m} / \mathrm{yr})$

R014 3 Saturated zone hydraulic gradient

$1.000 E-03 \div 1.000 E-033$

$31.600 E+00^{3} 1.500 E+00^{3}$

$34.000 E-01^{3} 4.000 E-013$

$32.500 E-0132.000 E-013$

$31.500 E-01^{3} 2.000 E-01^{3}$

$35.530 E+03^{3} 1.000 E+02^{3}$

$31.250 E-0332.000 E-023$

R014 Saturated zone b parameter

3 not used $3.300 E+00^{3}$ 


\section{ATTACHMENT 2}

IRESRAD, Version $6.3 \quad T \ll$ Limit $=180$ days $\quad 02 / 12 / 2007 \quad 09: 15$ Page 5 Summary: 126-F-1 Southern Portion Radionuclides Decayed to 2007 File: 126-F-1_Decayed_To_2007.RAD

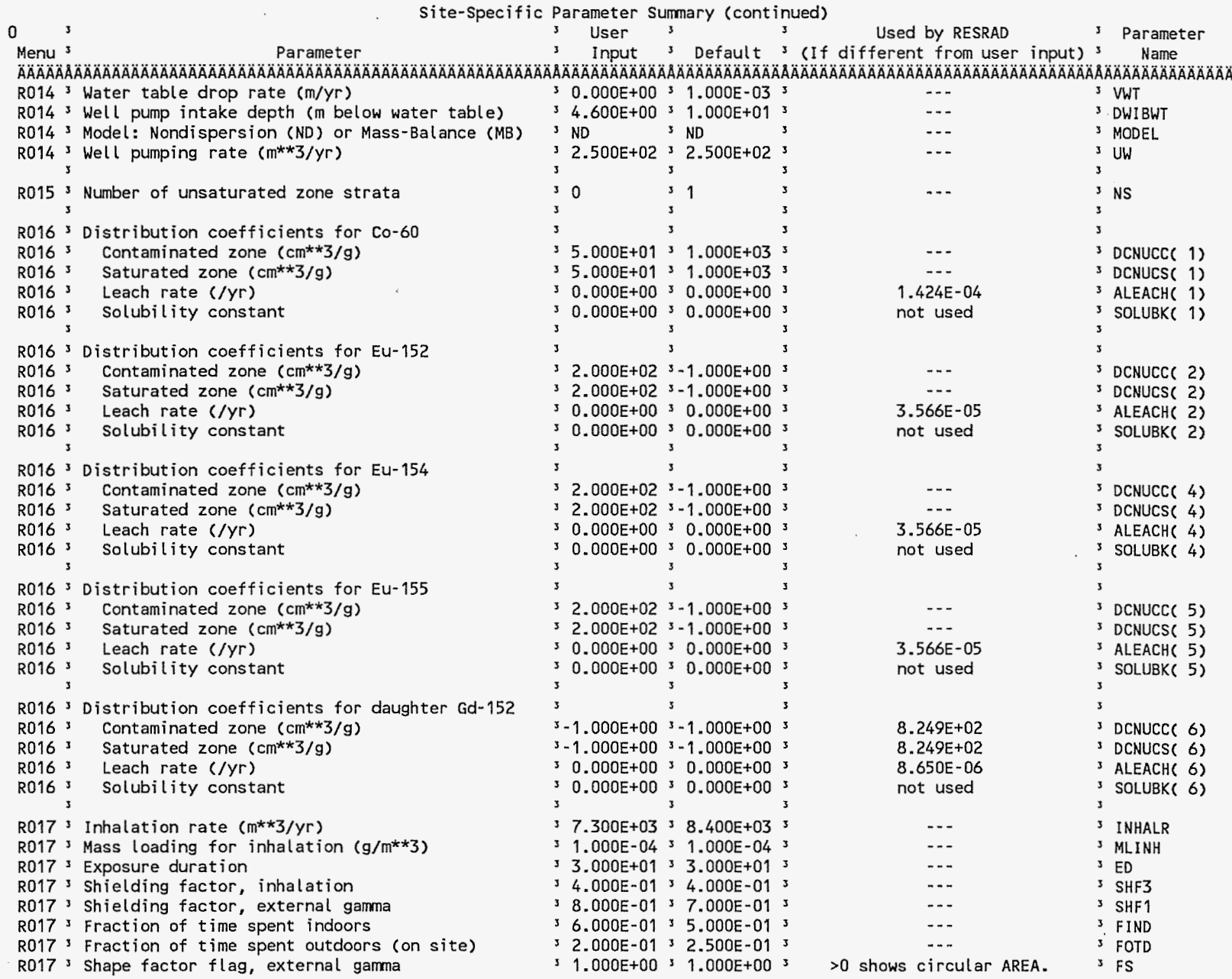

Attachment Originators: S. W. Clark Chk'd By M.W. Perrott Calc. No. 0100B-CA-V0102
Sheet No. $\underline{5}$ of $\underline{20}$ Date Date Rev. No. 


\section{ATTACHMENT 2}

1RESRAD, Version 6.3 T« Limit $=180$ days $\quad 02 / 12 / 2007 \quad 09: 15$ Page 6 Summary : 126-F-1 Southern Portion Radionuclides Decayed to 2007

File: 126-F-1_Decayed_To_2007.RAD

0

Parameter

Site-Specific Parameter Sumary (continued)

Menu ${ }^{3}$

3 User 3 Used by RESRAD

Used by RESRAD 3 Parameter

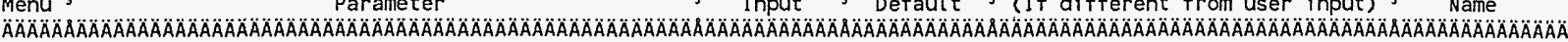
R017' Radi $i$ of shape factor array (used if $F S=-1$ ): ,

R017 s Outer annular radius (m), ring 1 :

R017 3 Outer annular radius (m), ring 2 :

$\mathrm{R}_{017^{3}}{ }^{3}$ Outer annular radius $(\mathrm{m})$, ring 3 :

R017 3 Outer annular radius (m), ring 4:

$\mathrm{R}_{017^{3}}{ }^{3}$ Outer annular radius $(\mathrm{m})$, ring 5 ;

$R 017^{3}$ Outer annular radius $(\mathrm{m})$, ring 6 :

3 3 3

not used 3 5.000E+01

s not used $37.071 E+013$

3 not used $30.000 E+003$

3 not used $50.000 E+003$

3 not used $30.000 E+00^{3}$

3 not used $30.000 E+00^{3}$

3 not used $30.000 E+00^{3}$

3 not used $30.000 E+003$

3 not used $30.000 E+00^{3}$

3 not used $30.000 E+003$

3 not used $30.000 E+00^{3}$

3 not used s $0.000 E+00$ s

Outer annular radius (m), ring

R017

outer annular radius $(\mathrm{m})$, ring 12 :

R017 3 Fractions of annular areas within AREA:

R017 3 Ring 1

R017 3 Ring ?

3 not used $31.000 E+00^{3}$

3 not used $32.732 E-013$

3 not used $30.000 E+00^{3}$

3 not used $30.000 E+003$

3 not used $30.000 E+00$ '

3 not used $30.000 E+00^{3}$

3 not used $30.000 E+00^{3}$

3 not used $30.000 E+00^{3}$

3 not used $30.000 E+00$ s

3 not used $30.000 E+00^{3}$

3 not used $30.000 E+00^{3}$

3 not used $30.000 E+00$ s

R017 3 Ring 10

R017' Ring 11

3

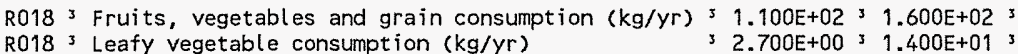

R018 3 Leafy vegetable consumption $(\mathrm{kg} / \mathrm{yr})$

$\begin{array}{llll}2.700 E+00 & 3 & 1.400 E+01 & 3 \\ 3 & 1.000 E+02 & 3 & 9.200 E+01\end{array}$

$3.600 \mathrm{E}+0136.300 \mathrm{E}+01^{3}$

$31.970 E+0135.400 E+003$

R018 Meat and poultry consumption ( $\mathrm{kg} / \mathrm{yr}$ )

R018 3 Fish consumption $(\mathrm{kg} / \mathrm{yr}$ )

R018 3 other seafood consumption ( $\mathrm{kg} / \mathrm{yr}$ )

R018 3 Soil ingestion rate $(g / y r)$

$\mathrm{R} 018$ S Drinking water intake ( $\mathrm{L} / \mathrm{yr}$ )

$3.000 E-0139.000 E-013$

$7.300 E+0133.650 E+01$ ?

$7.300 \mathrm{E}+02$ 3. $5.100 \mathrm{E}+02$ 3

$31.000 E+0031.000 E+00^{3}$

3 not used $31.000 E+003$

of hrinking water

R018 3 Contamination fraction of livestock water

R018 3 Contamination fraction of irrigation water

R018 Contamination fraction of aquatic food

R018 3 Contamination fraction. of plant food

R018 3 Contamination fraction of meat

R018 3 Contamination fraction of $\mathrm{milk}$

$31.000 E+0031.000 E+00$ s

s $1.000 E+00$ s $1.000 E+00$ s

3 $5.000 \mathrm{E}-01^{3} 5.000 \mathrm{E}-01^{3}$

$3-1$

$3-1$
$3-1$
$3-1$

$3-1$

R019 3 Livestock fodder intake for meat ( $\mathrm{kg} /$ day)

R019 3 Livestock fodder intake for milk (kg/day)

R019 3 Livestock water intake for meat ( $L /$ day)

R019 I Livestock water intake for $\mathrm{milk}$ (L/day)

$36.800 E+01^{3} 6.800 E^{3}+01^{3}$

$6.800 E+0136.800 E+01$
$5.500 E+0135$
$5.000 E+01$

$5.000 E+01^{3} 5.000 E+01^{3}$

$1.600 E+0231.600 E+023$

R019 3 Livestock soil intake ( $\mathrm{kg} /$ day)

3 5.000E-01 3 5.000E-01'

\begin{tabular}{|c|c|c|}
\hline$\cdots$ & 3 & RAD SHAPE (1) \\
\hline -. & $s$ & RAD_SHAPE ( 2) \\
\hline$\cdots$ & 3 & RAD_SHAPE( 3) \\
\hline$\cdots$ & 5 & RAD_SHAPE ( 4) \\
\hline$\cdots$ & 3 & RAD_SHAPE ( 5) \\
\hline$\cdots$ & 3 & RAD_SHAPE( 6) \\
\hline$\cdots$ & 3 & RAD_SHAPE ( 7) \\
\hline$\cdots$ & 3 & RAD_SHAPE ( 8) \\
\hline$\cdots$ & 3 & RAD_SHAPE( 9) \\
\hline$\cdots$ & 3 & RAD_SHAPE $(10)$ \\
\hline$\cdots$ & 3 & RAD_SHAPE (11) \\
\hline \multirow[t]{3}{*}{$\cdots$} & 3 & RAD_SHAPE (12) \\
\hline & 3 & \\
\hline & 3 & \\
\hline - n & 3 & FRACAC (1) \\
\hline$\cdots$ & 3 & FRACA ( 2) \\
\hline$\cdots$ & 3 & FRACA( 3) \\
\hline$\cdots$ & 3 & FRACA ( 4) \\
\hline$\cdots$ & 3 & FRACAC 5) \\
\hline$\cdots$ & 5 & FRACA( 6) \\
\hline$\cdots$ & 3 & FRACA ( 7) \\
\hline$\cdots$ & 3 & FRACA ( 8) \\
\hline$\cdots$ & 3 & FRACA ( 9) \\
\hline$\cdots$ & 3 & FRACA(10) \\
\hline$\cdots$ & 3 & FRACA(11) \\
\hline \multirow[t]{2}{*}{--} & 3 & FRACA(12) \\
\hline & 3 & \\
\hline$\cdots$ & 3 & DIET(1) \\
\hline$\cdots$ & 3 & $\operatorname{DIET}(2)$ \\
\hline$\cdots$ & 3 & $\operatorname{DIET}(3)$ \\
\hline$\cdots$ & 3 & DIET (4) \\
\hline-- & 3 & $\operatorname{DIET}(5)$ \\
\hline-- & 3 & $\operatorname{DIET}(6)$ \\
\hline$\cdots$ & 3 & SOIL \\
\hline$\cdots$ & 3 & DWI \\
\hline$\cdots$ & 3 & FDW \\
\hline$\cdots$ & 3 & FHHW \\
\hline$\cdots$ & 3 & FLW \\
\hline 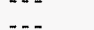 & y & FIRW \\
\hline $500 E+00$ & 3 & $\begin{array}{l}\text { FR9 } \\
\text { FPIANT }\end{array}$ \\
\hline $100 \mathrm{E}+01$ & 3 & $\begin{array}{l}\text { PPLAN1 } \\
\text { FMEAT }\end{array}$ \\
\hline $100 E+01$ & 3 & FMILK \\
\hline & 3 & \\
\hline$-\cdots$ & 3 & LF15 \\
\hline-- & 3 & LF16 \\
\hline$\cdots$ & 3 & LW15 \\
\hline$\cdots$ & 3 & LW16 \\
\hline$\cdots$ & 3 & LSI \\
\hline
\end{tabular}




\section{ATTACHMENT 2}

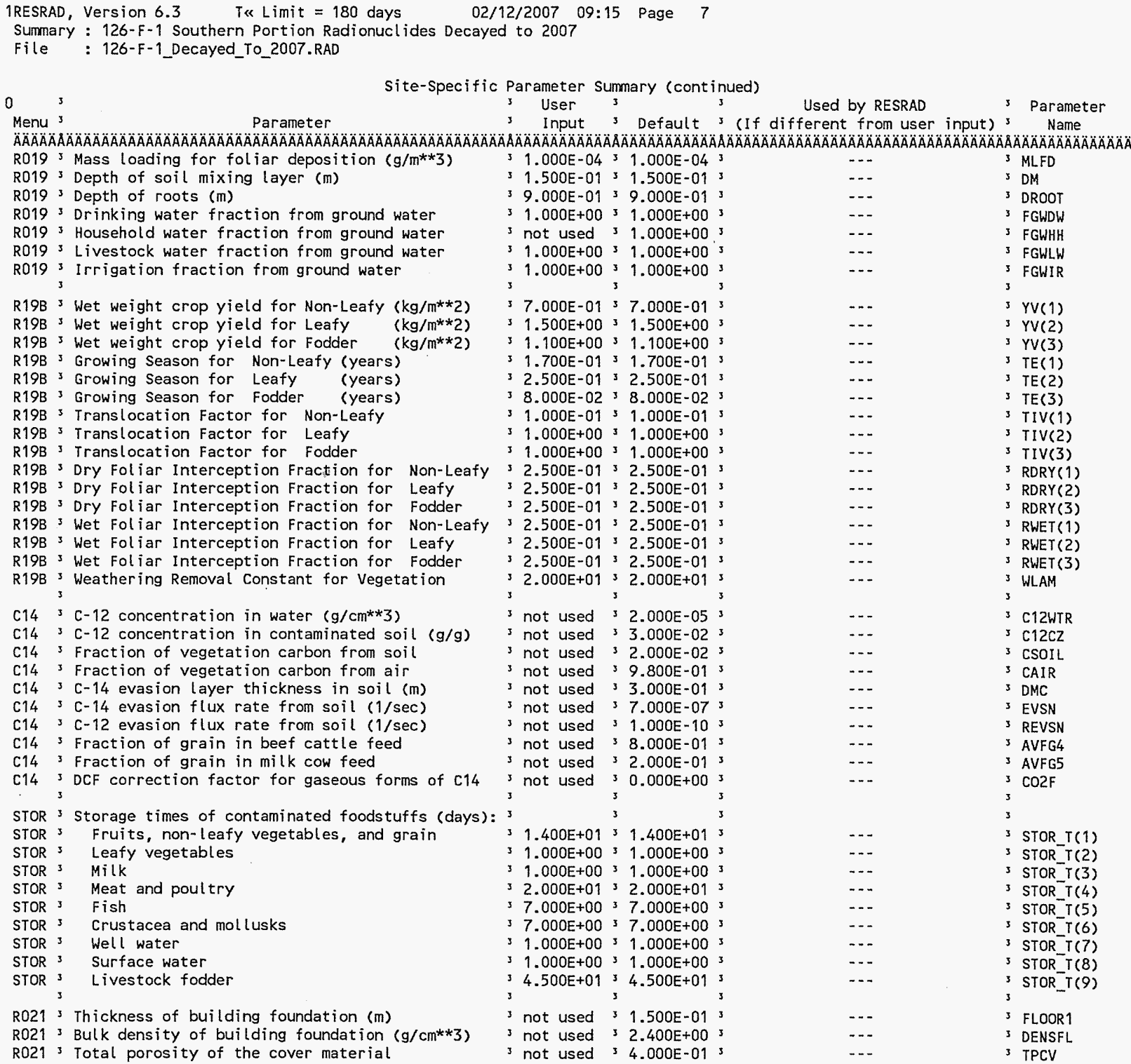

Attachment Originators: S.W. Clark Chk'd By M.W. Perrott

Calc. No. 0100B-CA-V0102
Sheet No. 7 of 20 Rev. No. 


\section{ATTACHMENT 2}

1RESRAD, Version $6.3 \quad$ T« Limit $=180$ days $02 / 12 / 2007 \quad 09: 15$ Page 8

Summary : 126-F-1 Southern Portion Radionuclides Decayed to 2007

File: 126-F-1_Decayed_To_2007.RAD

\section{0}

$0{ }^{3}$ Site-Specific $\underset{3}{\text { Parameter Summary (continued) }}$

3 Input

Used by RESRAD 3 Parameter

Default ${ }^{3}$ (If different from user input) ${ }^{3}$ Name

R021 Total porosity of the building foundation 3 not used $31.000 \mathrm{E}-01^{3}$, 3

R021 s volumetric water content of the cover material s not used $35.000 \mathrm{E}-02 \mathrm{~s}$

R021 s Volumetric water content of the foundation s not used s 3.000E-02 3

R021 s Diffusion coefficient for radon gas (m/sec)

R021 3 in cover material

RO21 3 in foundation material

R021 3 in contaminated zone soil

R021 3 Radon vertical dimension of mixing (m)

R021 3 Average building air exchange rate $(1 / \mathrm{hr})$ 3

not used $3.000 \mathrm{E}^{-06}$

3 not used $33.000 E-07^{3}$

3 not used 32.000 E-063

not used $s 2.000 E+00 \mathrm{~s}$

3 not used $35.000 E-013$

3 not used $32.500 E+003$

R021 3 Height of the building (room) (m)

R021 3 Building interior area factor

Building depth below ground surface (m)

3 not used $30.000 E+00$ s

3 not used $3-1.000 E+00^{3}$

3 not used $32.500 E-01^{3}$

3 not used $31.500 E-013$

nower of $\mathrm{Rn}-220$ gas

3

TITL 's Number of graphical time points

TITL ${ }^{3}$ Maximum number of integration points for dose

TITL 3 Maximum number of integration points for risk

Maximum number of integration points for risk

$\begin{array}{rrrr} & 3 & & 3 \\ 64 & 3 & \ldots & 3 \\ 5 & 3 & \ldots & 3 \\ 17 & 3 & \ldots & 3\end{array}$

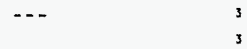

3 DIFCV

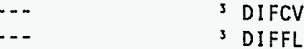

$\begin{array}{ll}-.- & 3 \text { DIFFL } \\ -- & 3 \text { DIFCZ }\end{array}$

3 HMIX

- 2 REXG

-

-

3 DMFL

-.. 3 EMANA(1)

-.- 3 EMANA(2)

Summary of Pathway Selections

\section{Pathway}

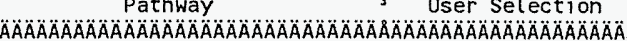

1 - external gamma

2 -- inhalation (w/o radon

3 -- plant ingestion

4 -. meat ingestion

5 -. milk ingestion

6 -- aquatic foods

7 -- drinking water

8 -. soil ingestion

9 -. radon

Find peak pathway doses

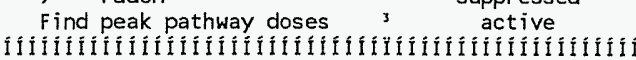

active

active

active

active

active

active

active

active
3 NPTS

3 LYMAX

$\left(-\frac{10}{20}\right.$

$\cdots$

IIIIIIIIIIIIIII

Attachment 2

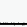
Sheet No. 8 of 20

Chk'd By M.W. Perrott Date

Calc. No. 0100B-CA-V0102 Date Rev. No. 


\section{ATTACHMENT 2}

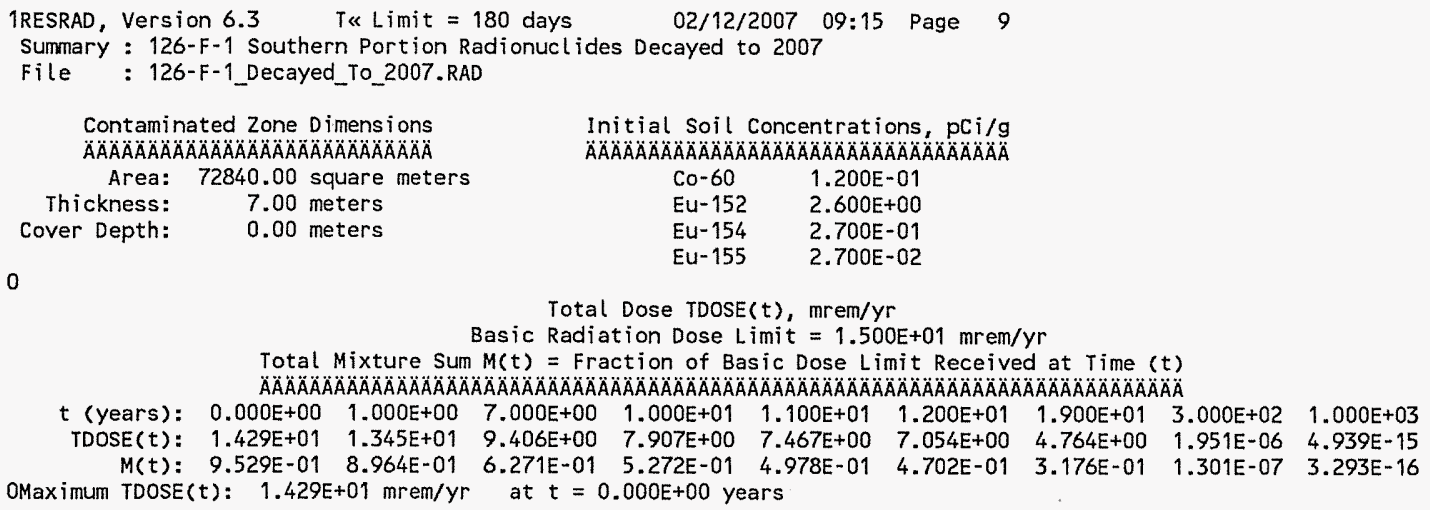

$t$ (years) : $\quad \begin{array}{llllllllll}0.000 E+00 & 1.000 E+00 & 7.000 E+00 & 1.000 E+01 & 1.100 E+01 & 1.200 E+01 & 1.900 E+01 & 3.000 E+02 & 1.000 E+03\end{array}$

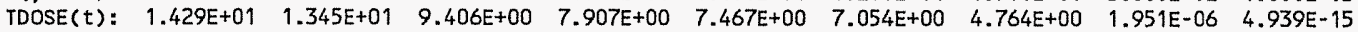
$M(t): \begin{array}{rlllllllll} & 9.529 E-01 & 8.964 E-01 & 6.271 E-01 & 5.272 E-01 & 4.978 E-01 & 4.702 E-01 & 3.176 E-01 & 1.301 E-07 & 3.293 E-16\end{array}$ OMaximum $\operatorname{TDOSE}(t): 1.429 E+01 \mathrm{mrem} / \mathrm{yr}$ at $t=0.000 E+00$ years 


\section{CALCULATION COVER SHEET}

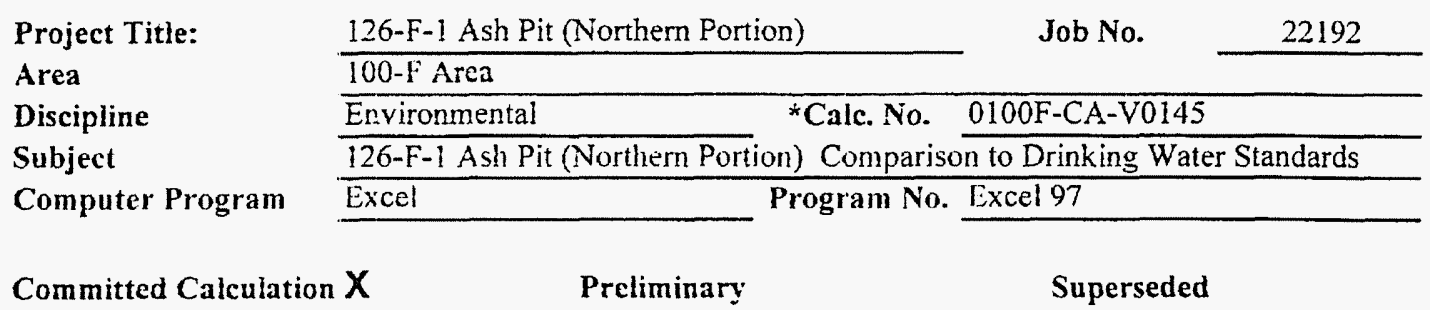

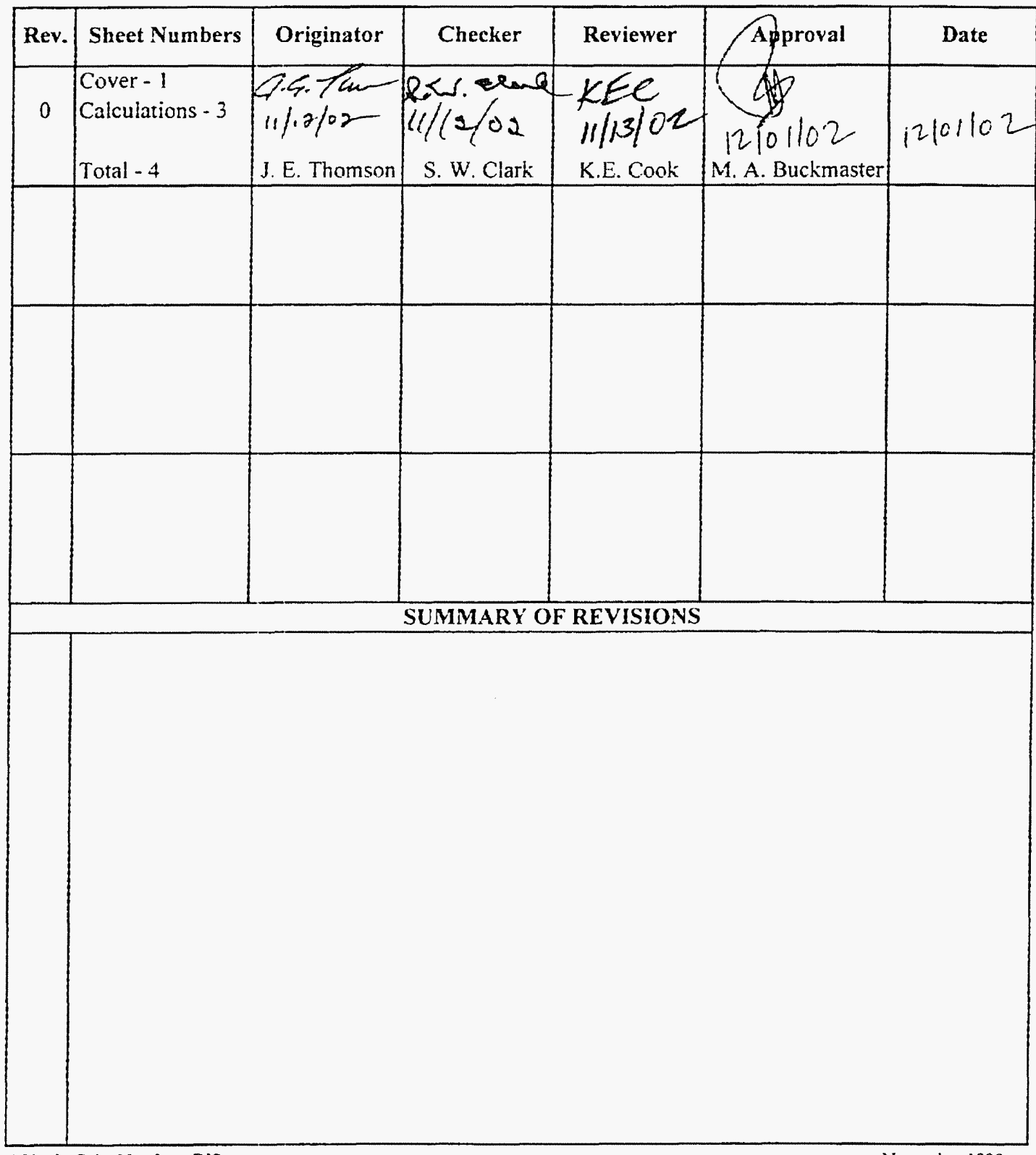


Originator J.E.Thomson Date

Purpose:

Compare RESRAD derived groundwater radionuclide concentrations to maximum contamination levels (MCLs) (summarized in DOE 2001, the Remedial Design Report/Remedial Action Work Plan for the 100 Area (RDR/RAWP), DOE/RL-96-17, Rev. 3, Table 2-3). Compare beta/gamma emitter dose contribution to the maximum allowable dose of $4 \mathrm{mrem} / \mathrm{yr}$ ( 40 CFR Part 141, Subpart $B, 141.16$ ), as calculated using NBS Handbook 69 individual organ-dose calculation methodology and EPA-570/9-76-003 total organ-dose calculation methodology.

Table of Contents:

1. Calculation Summary

2. Comparison to MCLs

4. Cumulative Dose Comparison

Given/References:

1) RESRAD derived groundwater radionuclide concentrations from Calc. No. 0100F-CA-V0145, Rev. 0, 126-F-1 Ash Pit (Northem Portion) RESRAD Calculation.

2) Radionuclide concentrations in water corresponding to $4 \mathrm{mrem} / \mathrm{year}\left(\mathrm{C}_{4} \mathrm{mrem} / \mathrm{yr}\right)$ from Calc. No. $0100 \mathrm{X}$-CA-V0128 or as determined from references in item 5 .

3) MCLs summarized in Table 2-3 of: DOE, 2001, Remedial Design ReportRemedial Action Work Plan for the 100 Area (RDR/RAWP), DOE/RL-96-17, Rev. 3, U.S. Department of Energy, Richland Operations office, Richland, WA.

4) Maximum allowable dose (beta/gamma emitters) from: 40 CFR 141, "National Primary Drinking Water Regulation," Code of Federal Regulations, as amended.

5) Individual organ-dose calculation methodology from: NBS Handbook 69, Maximum Permissible Body Burdens and Maximum

Permissible Concentrations of Radionuclides in Air or Water for Occupational Exposure, as amended, U.S. Department of Commerce, Washington D.C., and EPA-570/9-76-003. National Interim Primary Drinking Water Regulations. U.S. Environmental Protection Agency, Office of Water Supply. Washington D.C.

Solution:

1. The site data for the calculation are the groundwater concentrations for the COCs (daughter products are not considered) over time from the RESRAD groundwater (GW) concentration file.

2. If the site conceptual model breaks the contamination into multiple layers with differing concentrations, then the GW concentrations from the various model runs are added (for each time interval) to provide the concentration data for comparison to the individual $\mathrm{MCLs}$ and the dose calculation for the beta and gamma emitters.

3. Compare the summed concentrations for each radionuclide to the GW MCL given in the RDR/RAWP, Table 2-3.

4. The cumulative dose for each organ for all beta and gamma emitting COCs ( Cs-137, Cv-60) at time $t$ is calculated separately using the activity corresponding to a 4 mrem/year dnse $\left(r_{4}\right)$ and the sum of fractions equation shown below (from EPA-570/9-76-003). The organs for which doses need to be computed are total body, bone, liver and gastrointestina! tract [lower large intestine] (GI(LLI)). The individual organ doses are compared to $4 \mathrm{mrem} / \mathrm{yr}$.

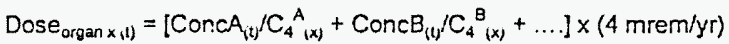

Where:

Dose $_{\text {orgen } x(1)}$ is the total dose to organ $x$ in mrem/yr

Conc $A_{(1)}$ is the concentration of isotope $A$ at time $t$ in $p C i / L$

$\mathrm{C}_{4}{ }^{\mathrm{A}}(\mathrm{x})$ is the $4 \mathrm{mrem} / \mathrm{yr}$ dose equivalent concentration for organ $\mathrm{x}$ of isotope $\mathrm{A}$ at time $t$ in $\mathrm{pCi} / \mathrm{L}$

If the dose for organ $x \leq 4 \mathrm{mrem} / \mathrm{yr}$, then the standard is met.

Conclusions:

The summed concentrations for each radionuclide are less than the GW MCL given in the RDRRAWP.

The cumulative dose for each total body, bone, liver and gastrointestinal tract for all beta and gamma emitting COCs is less than 4 mrem/yr (using EPA-570/9-76-003 total dose calculation methodology). 


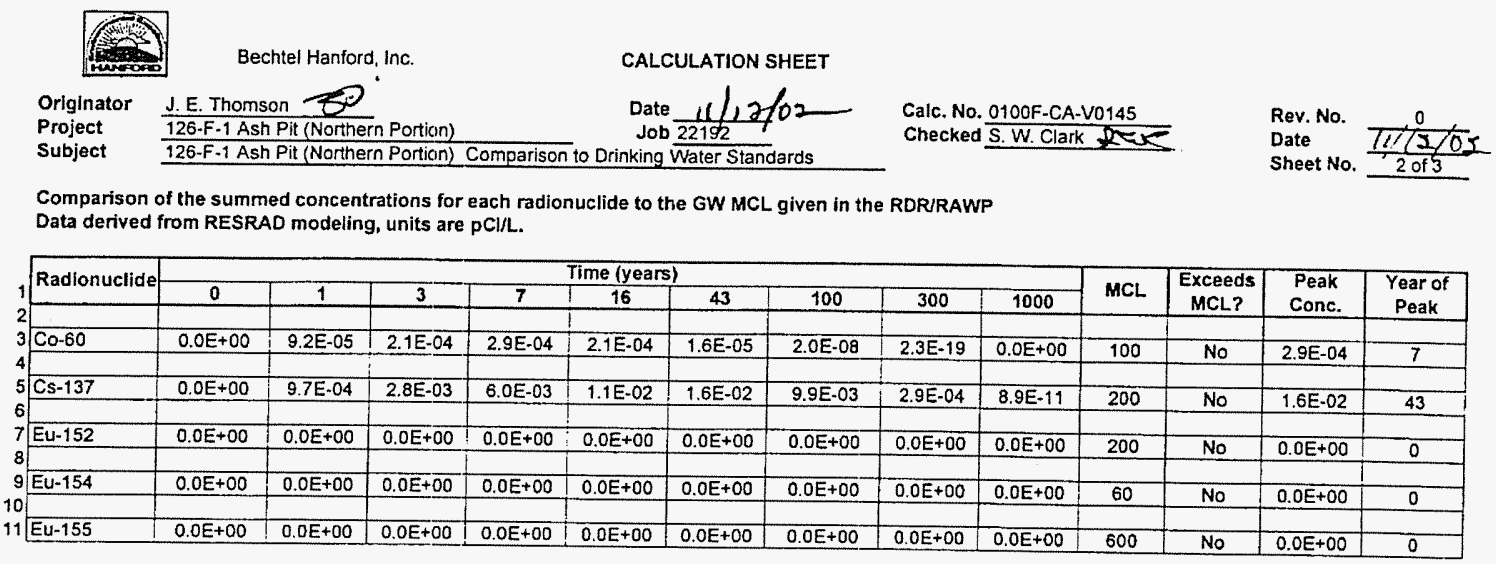




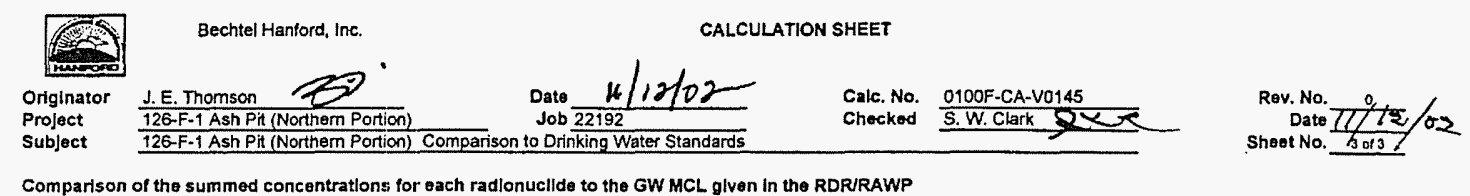
Data derived from RESRAD modelling, units are pClL. $C_{4}$ values from Calculation 0100X-CA-Voozs.
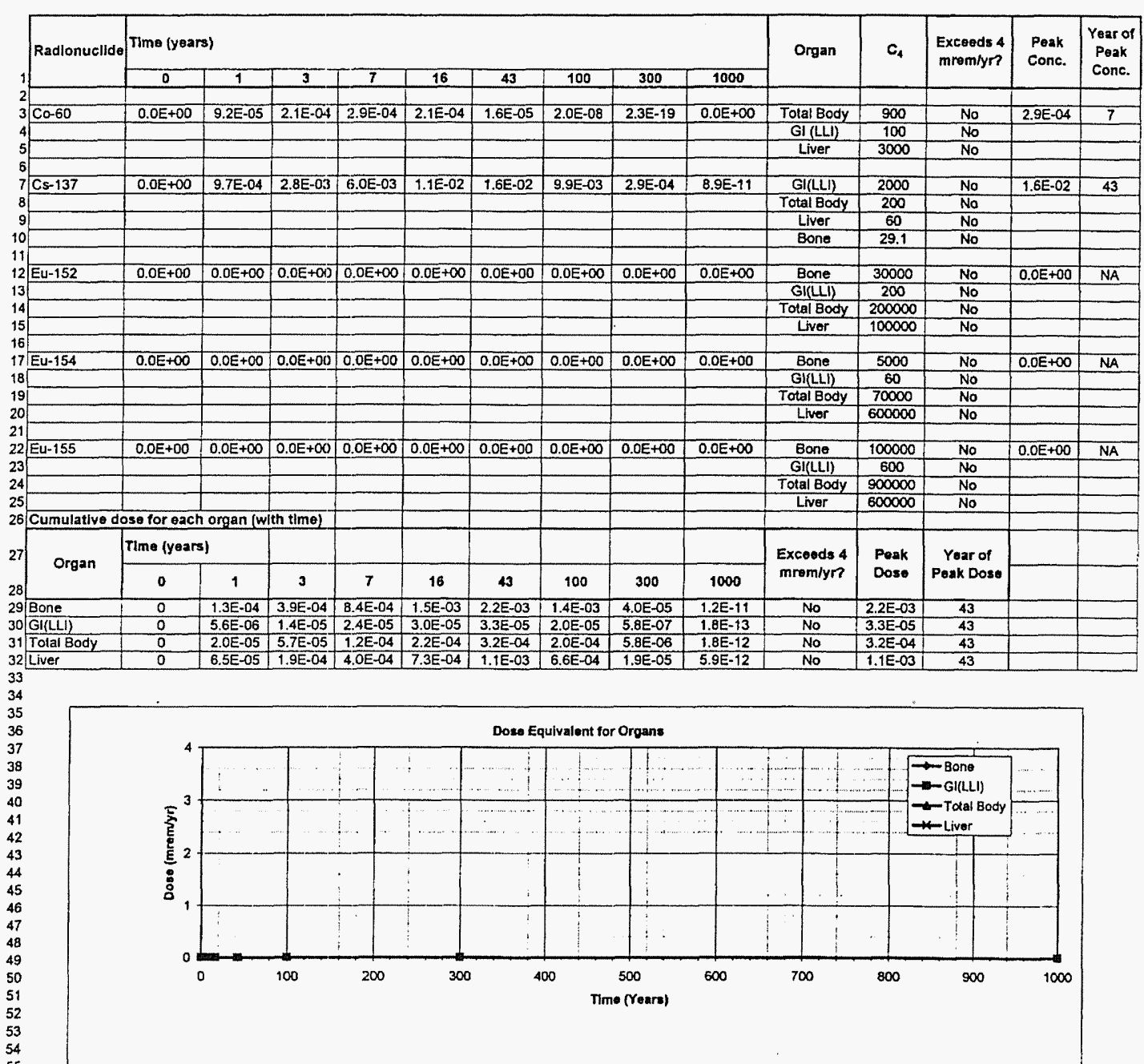


\section{CALCULATION COVER SHEET}

Project Title:

Area

Discipline

Subject

Computer Program
100-E* Remedial Action

Job No.

14655 100-F Area

Environmental

126-Fol (Southern Portion) Comparison to Drinking Water Standards

Program No. Excel 97

Committed Calculation $X$

Preliminary

Superseded

Voided

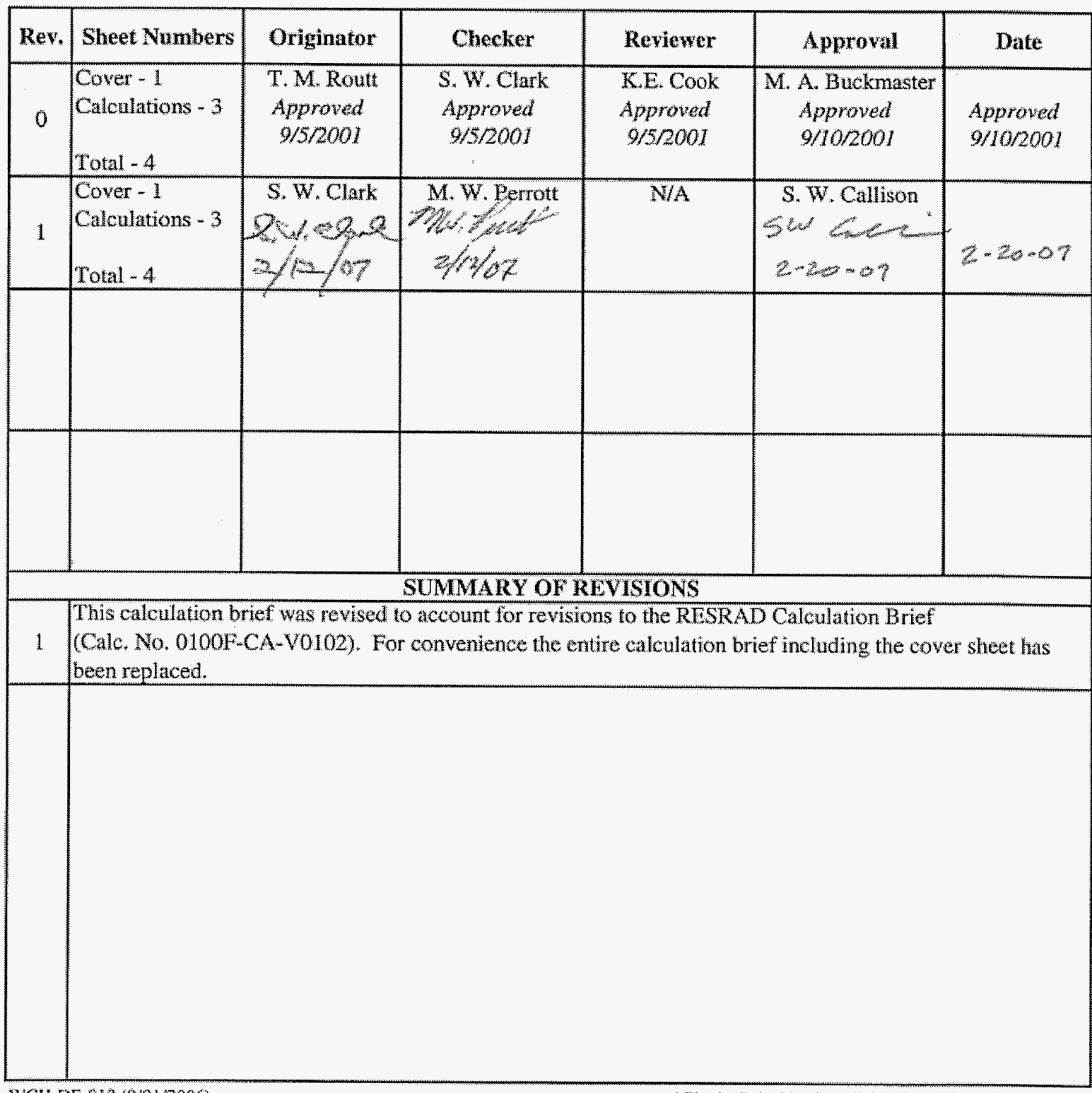


Washington Closure Hanford

Originator S.W. Clark $\mathrm{CW}$

Project $\frac{100-F \text { Remedial Action }}{100-F-1(S o u t h e n ~ P o n s ~}$

Subject

\section{CALCULATION SHEET}

Date $z / t z / 5 \%$ Calc. No. 0100F-CA-V0101

Job No. 14 (155

Checked M. W. Perrott

Rev. No. $\frac{1}{\text { Date }} \frac{1}{1 \text { /c: }}$
Sheet No. 3

Purpose:

Compare RESRAD derived groundwater radionuclide concentrations to MCLs (summarized in DOE 2000, the RDR/RAWP, DOE/RL-96-17, Rev. 5, Table 2-3). Compare alpha emitter dose contribution to the maximum allowable gross particle activity of $15 \mathrm{pCi} / \mathrm{L}$

(40 CFR Part 141, Subpart B, 141.15). Compare beta/gamma emitter dose contribution to the maximum allowable dose of $4 \mathrm{mrem} / \mathrm{yr}$ ( $40 \mathrm{CFR}$ Part 141 , Subpart B, 141.16), as calculated using NBS Handbook 69 individual organ-dose calculation methodology and EPA-570/9-76-003 total organ-dose calculation methodology.

Table of Contents:

1. Calculation Summary

2. Comparison to MCLS

3. Cumulative Dose Comparison

Given/References:

1) RESRAD derived groundwater radionuclide concentrations from Calc. No. 0100F-CA-V0102, Rev. 1, 126-F-1 (Southern Portion) RESRAD Calculation.

2) Remedial action goals for groundwater and MCLs summarized in Table 2-3 of the Remedial Design Report/Remedial Action Work Plan for the 100 Area (RDR/RAWP), DOE/RL-96-17, Rev. 5, U.S. Department of Energy, Richland Operations office, Richland, WA.

3) Maximum allowable dose of $4 \mathrm{mrem} / \mathrm{yr}$ for beta/gamma emitters and maximum allowable gross particle activity of $15 \mathrm{pCi} / \mathrm{L}$ for alpha emitters from: 40 CFR 141, "National Primary Drinking Water Regulation," Code of Federal Regulations, as amended (40 CFR Part 141, Subpart G, 141,66).

4) $1 / 25$ th of the derived concentration guide values from Radiation Protection of the Public and Environment, DOE Order 5400.5, U.S. Department of Energy, Washington D.C.

5) Individual organ-dose calculation methodology from: NBS Handbook 69, Maximum Permissible Body Burdens and Maximum Permissible Concentrations of Radionuclides in Air or Water for Occupational Exposure, as amended, U.S. Department of Commerce, Washington D.C., and EPA570/9-76-003, National Interim Primary Drinking Water Regulations, U.S. Environmental Protection Agency, Office of Water Supply, Washington D.C.

28 Solution:

1. The site data for the calculation are the groundwater concentrations for the COCs (daughter products are not considered) over time from the RESRAD groundwater (GW) concentration file.

2. If the site conceptual model breaks the contamination into multiple layers with differing concentrations, then the GW concentrations from the various model runs are added (for each time interval) to provide the concentration data for comparison to the individual MCLs and the dose calculation for the beta and gamma emitters.

3. Compare the summed concentrations for each radionuclide to the GW MCL given in the RDR/RAWP, Table 2-3.

4. The cumulative dose for each organ for all beta and gamma emitting COCs (Co-60, Eu-152, Eu-154, Eu-155) at time $t$ is calculated separately using the activity corresponding to a 4 mrem/year dose $\left(C_{4}\right)$ and the sum of fractions equation shown below (from EPA-570/9-76-003). The organs for which doses need to be computed are total body, bone, liver and gastrointestinal tract [lower large intestine] (Gl(LLI)). The individual organ doses are compared to $4 \mathrm{mrem} / \mathrm{yr}$.

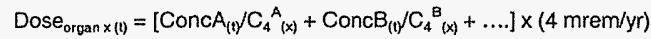

Where:

Dose $e_{\text {organ } x(1)}$ is the total dose to organ $x$ in mrem/yr

Conc $A_{(t)}$ is the concentration of isotope $A$ at time $t$ in $\mathrm{pCi} / \mathrm{L}$

$\mathrm{C}_{4}{ }^{\mathrm{A}}(\mathrm{x})$ is the $4 \mathrm{mrem} / \mathrm{yr}$ dose equivalent concentration for organ $\mathrm{x}$ of isotope $\mathrm{A}$ at time $\mathrm{t}$ in $\mathrm{pCi} / \mathrm{L}$

If the dose for organ $x \leq 4 \mathrm{mrem} / \mathrm{yr}$, then the standard is met.

Conclusions:

The summed concentrations for each radionuclide are less than the GW MCL given in the RDR/RAWP.

Alpha emitters are not a COC for this site.

The cumulative dose for each total body, bone, liver and gastrointestinal tract for all beta and gamma emitting COCs is less than 4 mrem/yr (using EPA570/9-76-003 total dose calculation methodology). 


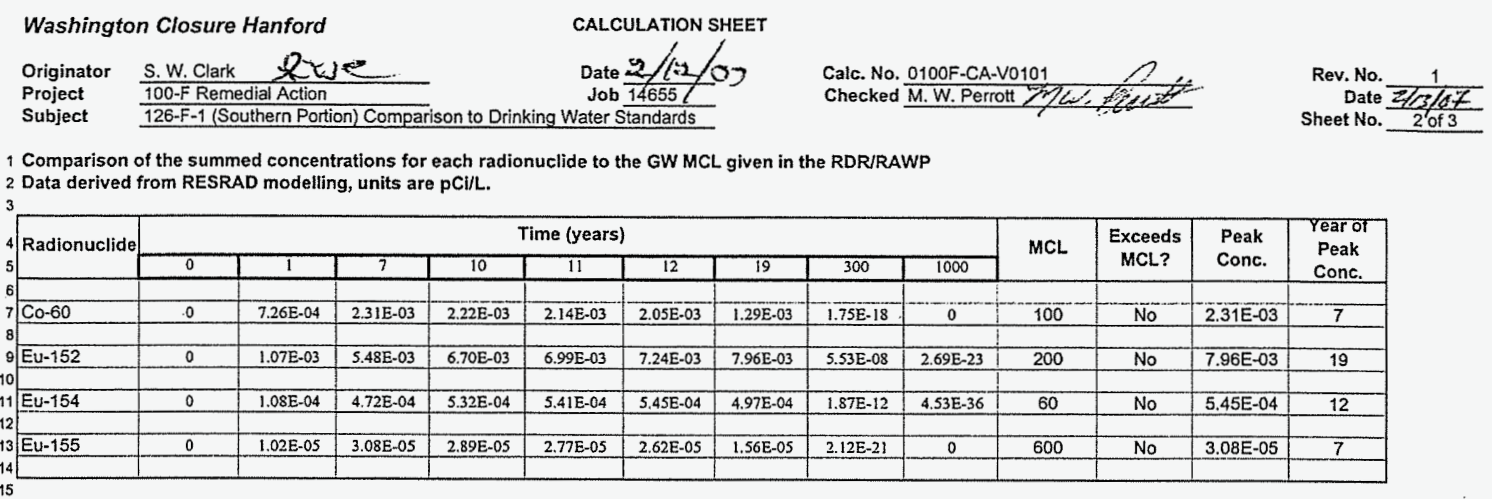




\section{Washington Closure Hanford}

Originator Project
Subject
S. W. Clark $\$$ 126-F-1 (Southern Portion) $100-F$ Remedial Action

CALCULATION SHEET

Conparison of the summed concentrations for each radionuclide to the GW MCL given in the RDR/RAWP Data derived from RESRAD modeling, units are $\mathrm{pCl} / \mathrm{L}, \mathrm{C}_{4}$ values from Calculation $0100 \mathrm{X}-\mathrm{CA}-\mathrm{V} 0025$

24. Cumulative dose for each organ (with time)

\begin{tabular}{|c|c|c|c|c|c|c|c|c|c|c|c|c|c|}
\hline \multirow{2}{*}{\multicolumn{2}{|c|}{ Organ }} & \multicolumn{9}{|c|}{ ime (years) } & \multirow{2}{*}{$\begin{array}{l}\text { Exceeds } 4 \\
\text { mrem/yr? }\end{array}$} & \multirow{2}{*}{ Peak Dose } & \multirow{2}{*}{$\begin{array}{c}\text { Year of } \\
\text { Peak } \\
\text { Dose }\end{array}$} \\
\hline & & 0 & 1 & 7 & 10 & 11 & 12 & 19 & 300 & 1000 & & & \\
\hline & Bone & $0.00 E+00$ & $2.29 E-07$ & $1.11 \mathrm{E}-06$ & $1.32 E-06$ & $1.37 E-06$ & 1.40E-06 & $1.46 \mathrm{E}-06$ & $7.37 \mathrm{E}-12$ & $3.59 \mathrm{E}-27$ & No & $1.46 \mathrm{E}-06$ & 19 \\
\hline & GI(LL) & $0.00 E+00$ & $5.77 \mathrm{E}-05$ & $2.34 \mathrm{E}-04$ & $2.58 E-04$ & $2.62 \mathrm{E}-04$ & $2.63 \mathrm{E}-04$ & $2.44 \mathrm{E}-04$ & $1.11 \mathrm{E}-09$ & $5.38 \mathrm{E}-25$ & No & $2.63 \mathrm{E}-04$ & 12 \\
\hline & Total Body & $0.00 \mathrm{E}+00$ & $3.25 E-06$ & $1.04 \mathrm{E}-05$ & $1.00 E-05$ & $9.68 \mathrm{E}-06$ & \begin{tabular}{|c|}
$9.29 \mathrm{E}-06$ \\
\end{tabular} & $5.92 \mathrm{E}-06$ & $1.11 \mathrm{E}-12$ & $5.38 E-28$ & No & $1.04 \mathrm{E}-05$ & 7 \\
\hline 0 & \begin{tabular}{|l} 
Liver \\
\end{tabular} & $0.00 \mathrm{E}+00$ & $1.02 E-06$ & $3.33 \mathrm{E}-06$ & $3.26 \mathrm{E}-06$ & \begin{tabular}{|l|}
$3.17 \mathrm{E}-06$ \\
\end{tabular} & $3.06 \mathrm{E}-06$ & $2.07 \mathrm{E}-06$ & $2.21 \mathrm{E}-12$ & $1.08 \mathrm{E}-27$ & No & $3.33 \mathrm{E}-06$ & 7 \\
\hline
\end{tabular}

\begin{tabular}{l|l|l|l|l|l|l|l|l|l|l|l}
\hline 30 & Liver & $0.00 E+00$ & $3.25 E-06$ & $1.04 E-05$ & $1.00 E-05$ & $9.68 E-06$ & $9.29 E-06$ & $5.92 E-06$ & $1.11 E-12$ & $5.38 E-28$ \\
\hline
\end{tabular}

34

35

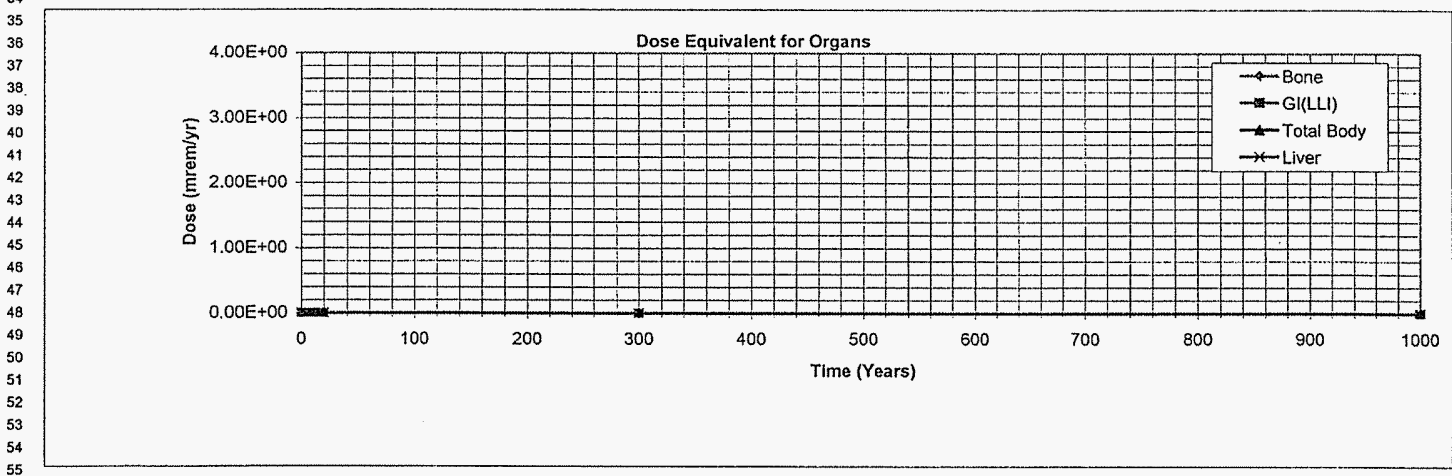


CVP-2002-00004

Rev. 1

C-82 


\section{DISTRIBUTION}

U.S. Department of Energy

Richland Operations Office

D. C. Smith (5)

A3-04

Fluor Hanford, Inc.

J. P. Shearer (WIDS)

E6-35

Washington Closure Hanford

S. W. Callison

$\times 9-07$

R. A. Carlson

$\times 9-07$

S. W. Clark

$\mathrm{H} 4-23$

L. A. Dietz

$\mathrm{H} 4-22$

L. M. Dittmer

$\mathrm{H} 4-23$

J. D. Fancher

$\mathrm{X} 9-07$

M. T. Hughes

$\mathrm{H} 4-23$

R. D. Meyers

X9-07

H. M. Sulloway

$\mathrm{H} 4-23$

Records and Document Control

H4-11

DOE-RL Public Reading Room

$\mathrm{H} 2-53$

Hanford Technical Library

P8-55 
CVP-2002-00004

Rev. 1

Distr-2 